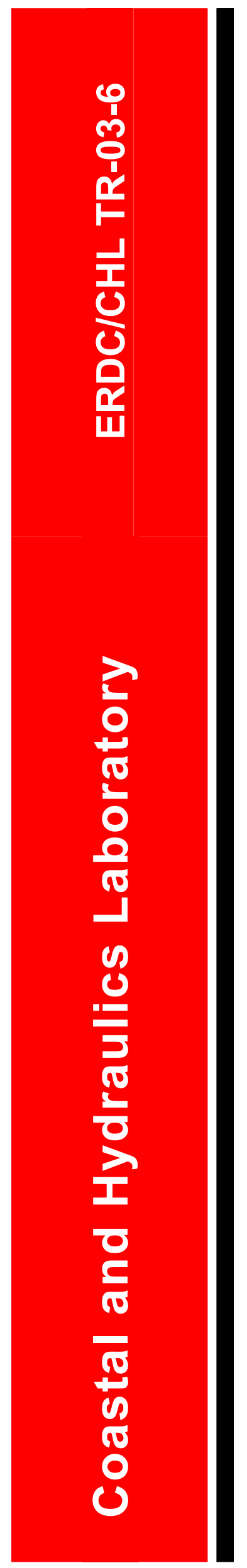

Coastal Inlets Research Program

\title{
Flow Table Study of Cook Inlet, Alaska
}

Steven A. Hughes and Gian-Marco Pizzo

July 2003

Approved for public release; distribution is unlimited. 


\section{Flow Table Study of Cook Inlet, Alaska}

by Steven A. Hughes

Coastal and Hydraulics Laboratory

U.S. Army Engineer Research and Development Center 3909 Halls Ferry Road

Vicksburg, MS 39180-6199

Gian-Marco Pizzo

Department of Civil and Environmental Engineering University of California at Berkeley

Berkeley, CA 34720

Final report

Approved for public release; distribution is unlimited.

Prepared for U.S. Army Corps of Engineers

Washington, DC 20314-1000

Under Scour at Inlet Structures Work Unit 32935 


\begin{abstract}
Complex flow patterns in upper Cook Inlet in the vicinity of the Port of Anchorage were simulated using a flow table. Initially, two idealized models of the inlet were constructed with terraced layers representing the bathymetry. Encouraging test results prompted construction of a model with three-dimensional bathymetry. Both ebb and flood maximum tide flows could be examined by reversing the models on the flow table. The intriguing flow patterns of Cook Inlet were visualized using floating particles and by dye injection. Strong horizontal and vertical mixing was evident through the model, particularly in the lee of headlands. The flow table models indicated that deposition of fine sediment at the Port of Anchorage may be caused, in part, by ebb flow separation at the upstream Cairn Point that creates a low-flow region at the port. Experimentation with the models also provided insight into potential improvements to dredge material disposal practices.

Theoretical analyses examined potential turbulence scale effects caused by geometric model distortion, and extensive experiments were conducted on the flow table to gauge the scale effects. Various jet flow geometries were tested at different model distortions, and velocity measurements in the region of flow separation were compared and evaluated. Results indicated that turbulence generated by vertical edges and manifested primarily in the horizontal plane had no distorted models where the vertical and horizontal turbulence had similar strength such as occurs with flow separation at a sloping edge. The difference was most noticeable near the bottom, whereas comparisons near the free surface were reasonable. The scale effect appeared to be localized around the boundary of the jet.
\end{abstract}

DISCLAIMER: The contents of this report are not to be used for advertising, publication, or promotional purposes. Citation of trade names does not constitute an official endorsement or approval of the use of such commercial products. All product names and trademarks cited are the property of their respective owners. The findings of this report are not to be construed as an official Department of the Army position unless so designated by other authorized documents. 


\section{Contents}

Conversion Factors, Non-SI to SI Units of Measurement................................ xiii

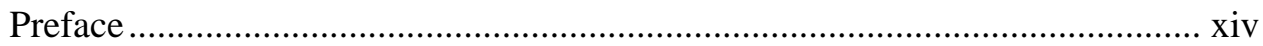

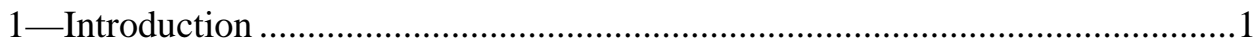

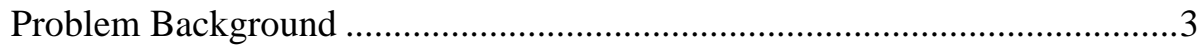

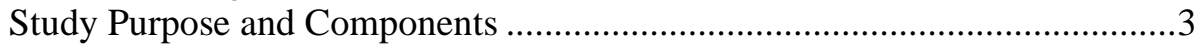

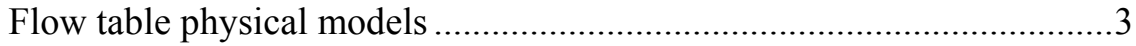

Turbulent scale effect in geometrically distorted physical models ...........4

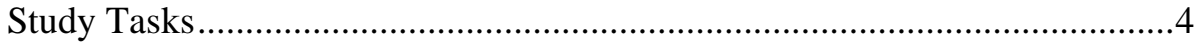

Task 1: Large-area idealized flow model of Cook Inlet ............................

Task 2: Small-area idealized flow model of Cook Inlet ............................5

Task 3: Turbulence scale effect in distorted physical models ....................5

Task 4: Effect of sloping transitions on flow hydrodynamics...................5

Task 5: Large-area 3-D flow model of Cook Inlet ....................................5

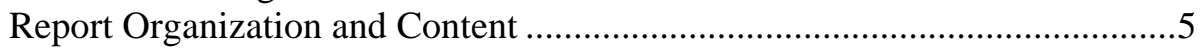

2-Coastal and Hydraulics Laboratory Flow Table ..........................................6

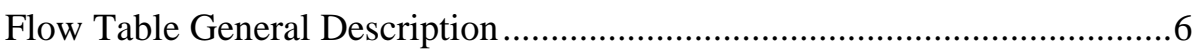

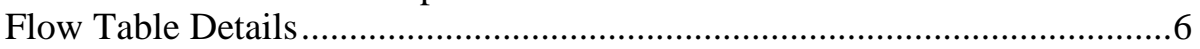

Flow Measurement System..................................................................

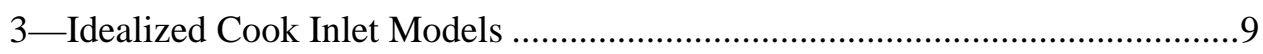

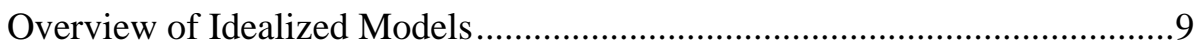

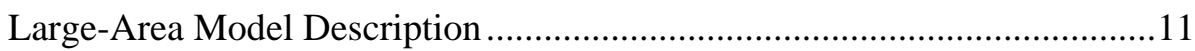

Large-Area Model Observations.............................................................14

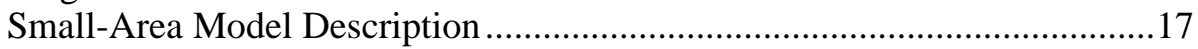

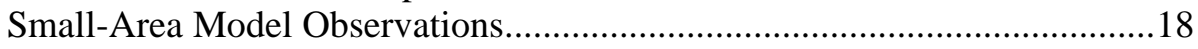

Conclusions From Idealized Models ........................................................18

4-Turbulence Scale Effect in Distorted Models...............................................23

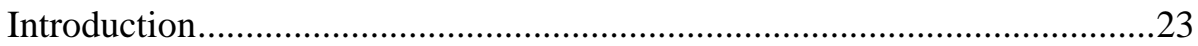

General Similitude Requirements ..........................................................23

Geometrically Distorted Models ................................................................24 
Turbulence Similitude in Geometrically Distorted Models .........................25

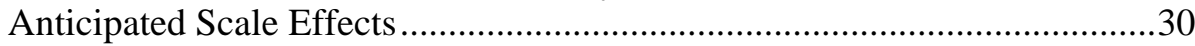

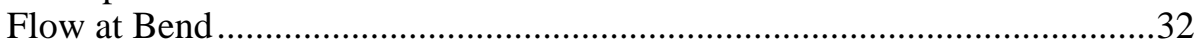

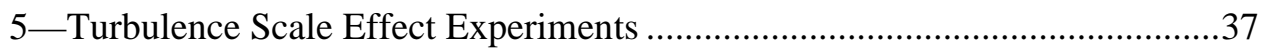

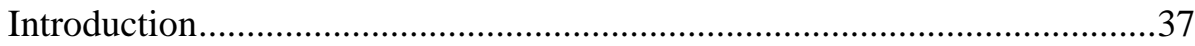

Case 1: Unconstrained flow separation at vertical edge........................37

Case 2: Constrained flow separation at vertical edge...............................37

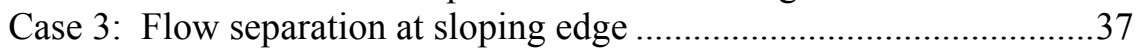

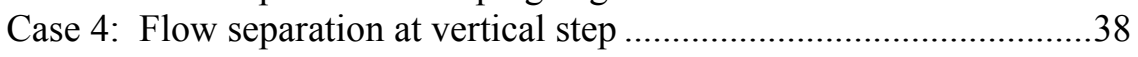

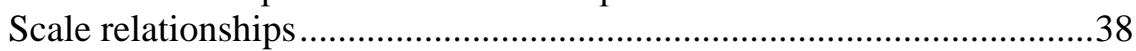

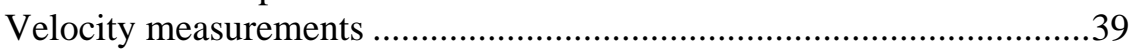

Case 1: Flow Separation at Vertical Edge - Free Jet......................................40

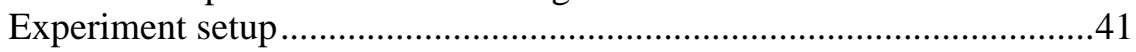

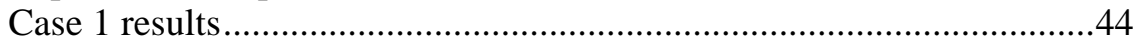

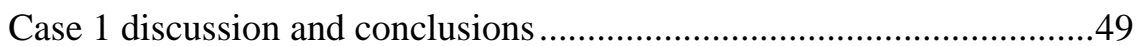

Case 2: Flow Separation at Vertical Edge - Constrained Jet .........................49

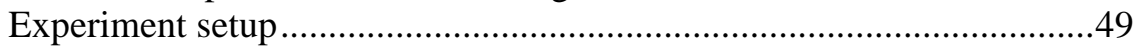

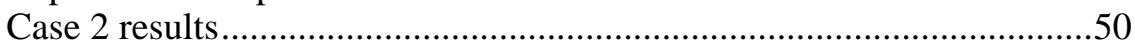

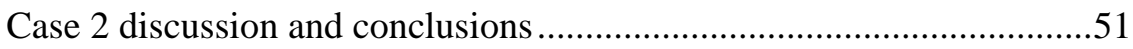

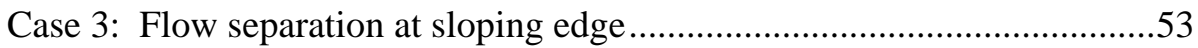

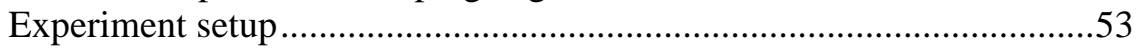

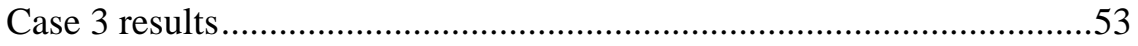

Case 3 discussion and conclusions .......................................................6

Case 4: Flow Separation at Vertical Step ..................................................63

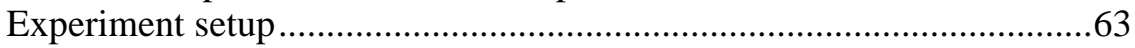

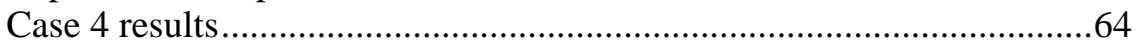

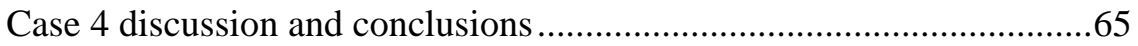

Conclusions from Turbulence Scale Effects Experiments............................65

6-Impact of Dredging Planform...............................................................67

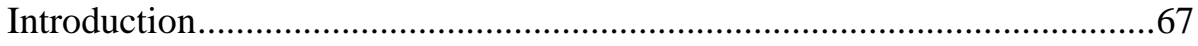

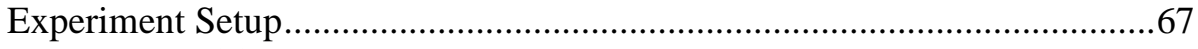

Dredge Transition Test Results....................................................................69

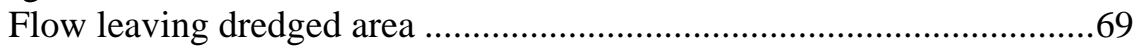

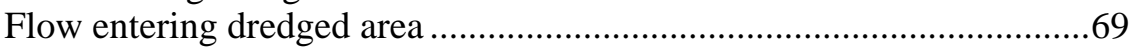

Conclusions from Dredge Transition Experiments....................................73

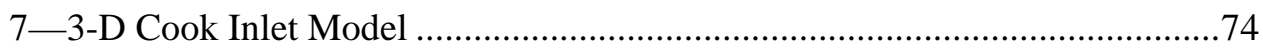

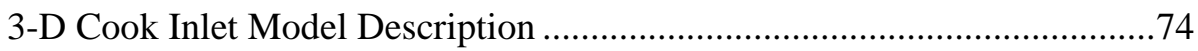

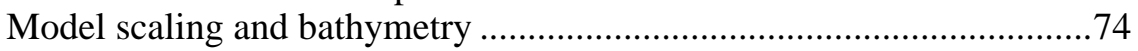

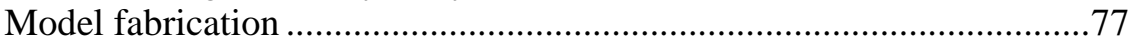

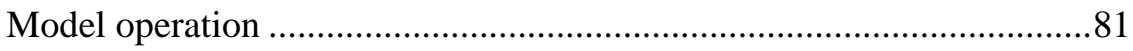

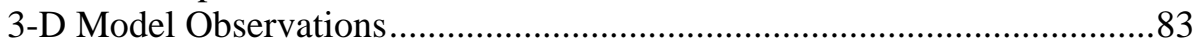

Conclusions from Cook Inlet 3-D Model ....................................................86 


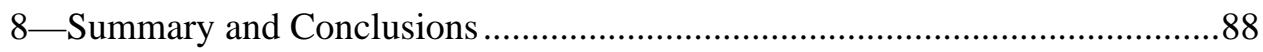

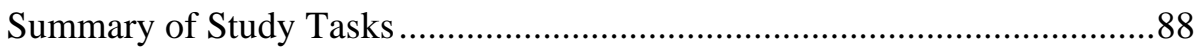

Task 1: Large-area idealized flow model of Cook Inlet........................88

Task 2: Small-area idealized flow model of Cook Inlet..........................88

Task 3: Turbulence scale effect in distorted physical models ................89

Task 4: Effect of sloping transitions on flow hydrodynamics................89

Task 5: Large-area 3-D flow model of Cook Inlet .................................89

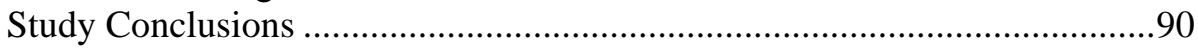

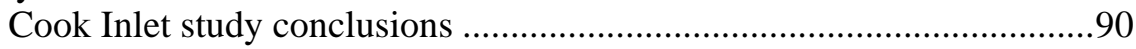

Turbulence scale effects study conclusions...........................................91

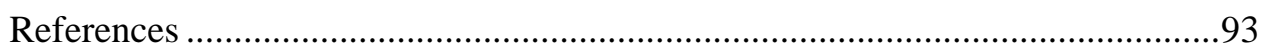

Appendix A-Case 1. Flow Separation at Vertical Edge - Free Jet...................A1

Appendix B-Case 2. Flow Separation at Vertical Edge - Constrained Jet....... B1

Appendix C—Case 3. Flow Separation at Sloping Edge ................................... 1

\section{List of Figures}

Figure 1. Cook Inlet, AK, location map ......................................................

Figure 2. Port of Anchorage, looking south ....................................................2

Figure 3. Side view of CHL flow table .....................................................

Figure 4. Schematic side view showing flow table components ........................ 7

Figure 5. View of traversing system looking down through glass bottom.........8

Figure 6. Area coverage of large-area and small-area idealized models..........10

Figure 7. Idealized bathymetry in large-area model........................................12

Figure 8. Cutting large-area model pieces with router ...................................13

Figure 9. Assembly of large-area model .....................................................13

Figure 10. Flood flow in large-area idealized model .......................................15

Figure 11. Flood flow separation at Point MacKenzie......................................15

Figure 12. Alaska District engineers observe dye patterns in large-area idealized model .......................................................................... 16

Figure 13. Cross channel flow at Point MacKenzie during flood tide ................16

Figure 14. Cutting small-area model pieces with router ...................................19 
Figure 15. Completed small-area model showing idealized bathymetry 19

Figure 16. Idealized bathymetry in small-area model .......................................20

Figure 17. Small-area idealized model positioning on flow table......................21

Figure 18. Turbulent flow cases tested on flow table..........................................38

Figure 19. Cross sections showing distortion for vertical edge tests .................41

Figure 20. Experiment setup for Case 1 free jet flow separation at vertical edge. .42

Figure 21. Velocity field $N_{Q}=1: 5$, prototype (Case 1) ....................................45

Figure 22. Velocity field $N_{Q}=1: 5$, distortion $=2$ (Case 1) ..............................45

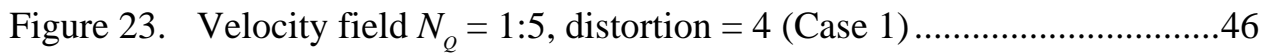

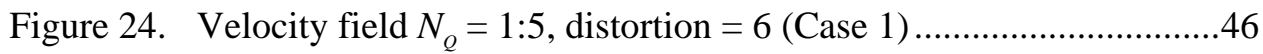

Figure 25. Comparison between prototype and distortion $=6, N_{Q}=1: 5$ (Case 1)

Figure 26. Velocity field NQ $=1: 5$, prototype vs. distortion $=6($ Case 1$) \ldots . . . .47$

Figure 27. Cross-flow velocity ratios, distortion $=2,4,6$ over prototype $\left(N_{Q}=1: 5\right.$, Case 1$)$

Figure 28. Principal flow direction velocity ratios, distortion $=2,4,6$ over prototype $\left(N_{Q}=1: 5\right.$, Case 1$)$

Figure 29. Experiment setup for Case 2 constrained jet flow separation at

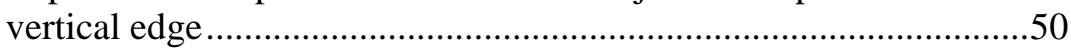

Figure 30. Velocity field $N_{Q}=1.0$, prototype vs. distortion $=6($ Case 2$) \ldots \ldots \ldots . .51$

Figure 31. Cross-flow velocity ratios, distortion $=2,4,6$ over prototype $\left(N_{Q}=1.0\right.$, Case 2). .52

Figure 32. Principal flow direction velocity ratios, distortion $=2,4,6$ over

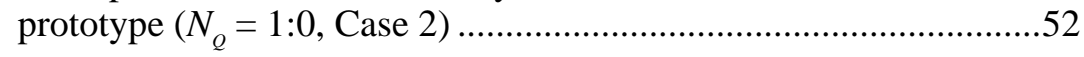

Figure 33. Cross sections showing distortion for sloping edge tests..................54

Figure 34. Velocity field at $2 / 3 d, N_{Q}=1.0$, prototype (Case 3) .......................54

Figure 35. Velocity field at $2 / 3 d, N_{Q}=1.0$, distortion $=2($ Case 3$) \ldots \ldots \ldots \ldots \ldots . . . . .55$

Figure 36. Velocity field at $2 / 3 d, N_{Q}=1.0$, distortion $=4$ (Case 3$) \ldots \ldots \ldots \ldots \ldots . . . .55$

Figure 37. Velocity field at $2 / 3 d, N_{Q}=1.0$, distortion $=6$ (Case 3$) \ldots \ldots \ldots \ldots \ldots . . .56$

Figure 38. Velocity field at $1 / 3 d, N_{Q}=1.0$, prototype (Case 3) ......................56

Figure 39. Velocity field at $1 / 3 d, N_{Q}=1.0$, distortion $=2($ Case 3$) \ldots \ldots \ldots \ldots \ldots . . . .57$

Figure 40. Velocity field at $1 / 3 d, N_{Q}=1.0$, distortion $=4($ Case 3$) \ldots \ldots \ldots \ldots \ldots . . . .57$

Figure 41. Velocity field at $1 / 3 d, N_{Q}=1.0$, distortion $=6($ Case 3$) \ldots \ldots \ldots \ldots \ldots . . . .58$

Figure 42 . Velocity field at $2 / 3 d, N_{Q}=1.0$, prototype vs. distortion $=6$ (Case 3). 
Figure 43. Velocity field at $1 / 3 d, N_{Q}=1.0$, prototype vs. distortion $=6$ (Case 3)

Figure 44. Cross-flow velocity ratios at $2 / 3 d$, distortion $=2,4,6$ over prototype $\left(N_{Q}=1.0\right.$, Case 3$)$

Figure 45. Principal flow direction velocity ratios at $2 / 3 d$, distortion $=2,4,6$ over prototype $\left(N_{Q}=1.0\right.$, Case 3$)$

Figure 46. Cross-flow velocity ratios at $1 / 3 d$, distortion $=2,4,6$ over prototype $\left(N_{Q}=1.0\right.$, Case 3$)$.

Figure 47. Principal flow direction velocity ratios at $1 / 3 d$, distortion $=2,4,6$ over prototype $\left(N_{Q}=1.0\right.$, Case 3$)$.

Figure 48. Experiment setup for Case 4 flow over a vertical step.

Figure 49. Comparison of horizontal flow magnitudes in lee of a vertical step65

Figure 50. Experiment setup for harbor dredging planform configuration.......68

Figure 51. Dredge transition planform configurations ..............................6 69

Figure 52. Flow leaving dredged region over vertical transition...................70

Figure 53. Flow leaving dredged region over sloping transition ....................70

Figure 54. Flow entering dredged region over vertical transition................... 71

Figure 55. Flow entering dredged region over sloping transition...................71

Figure 56. Photograph of flow entering dredged region over vertical

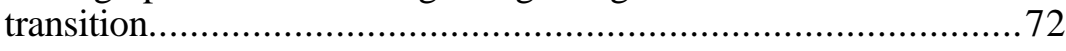

Figure 57. Photograph of flow entering dredged region over sloping transition............................................................................ 73

Figure 58. Area coverage of 3-D Cook Inlet model.................................75

Figure 59. Bathymetry of 3-D Cook Inlet model.................................... 77

Figure 60 . Router rough cut of bathymetry ........................................ 78

Figure 61 . Router finish cut of bathymetry .......................................... 79

Figure 62. Portion of 3-D model looking upstream ................................. 79

Figure 63. Portion of 3-D model looking downstream............................. 80

Figure 64. Close-up view looking downstream at Cairn Point.......................80

Figure 65. Close-up view looking downstream south of Port of Anchorage....81

Figure 66. 3-D Cook Inlet model installed for flood flow ......................... 82

Figure 67. Surface flow tracer showing reduced flow at Port of Anchorage during ebb tide

Figure 68. Dye injection showing reduced flow at Port of Anchorage downstream of Cairn Point.

Figure 69. Aerial showing approximate flow separation and entrainment region in lee of Cairn Point during ebb tide................................ 85

Figure A1. Case $1, Q=1.5 \mathrm{~L} / \mathrm{sec}$, prototype ................................... A2 


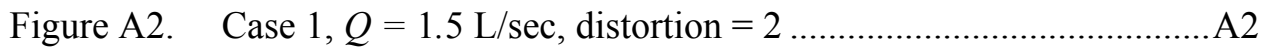

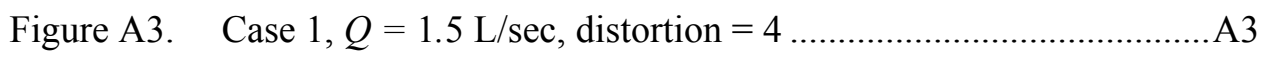

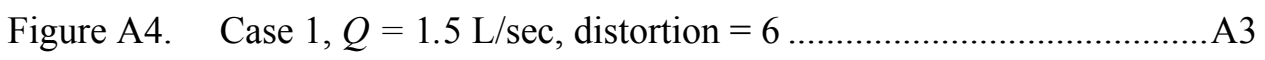

Figure A5. Case $1, Q=1.5 \mathrm{~L} / \mathrm{sec}$, prototype vs. distortion $=2 \ldots \ldots \ldots \ldots \ldots \ldots . . . \mathrm{A} 4$

Figure A6. Case $1, Q=1.5 \mathrm{~L} / \mathrm{sec}$, prototype vs. distortion $=4 \ldots \ldots \ldots \ldots \ldots \ldots . .$. A 4

Figure A7. Case $1, Q=1.5 \mathrm{~L} / \mathrm{sec}$, prototype vs. distortion $=6 \ldots \ldots \ldots \ldots \ldots \ldots . . . . . .5$

Figure A8. Case $1, Q=1.5 \mathrm{~L} / \mathrm{sec}$, prototype minus distortion $=2 \ldots \ldots \ldots \ldots \ldots .$. A 5

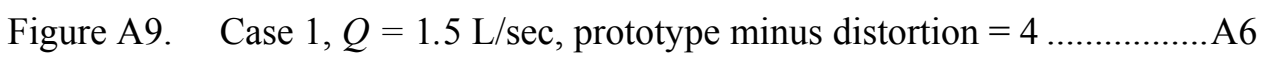

Figure A10. Case $1, Q=1.5 \mathrm{~L} / \mathrm{sec}$, prototype minus distortion $=6 \ldots \ldots \ldots \ldots \ldots$. A6

Figure A11. Case $1, Q=1.5 \mathrm{~L} / \mathrm{sec}$, ratios between prototype and

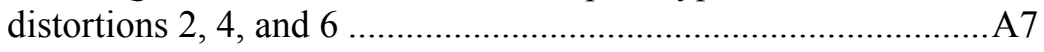

Figure A12. Case $1, Q=1.5 \mathrm{~L} / \mathrm{sec}$, ratios between prototype and

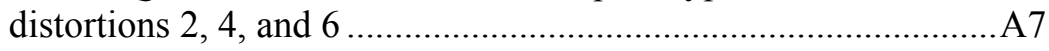

Figure A13. Case $1, Q=1.0 \mathrm{~L} / \mathrm{sec}$, prototype............................................. 8

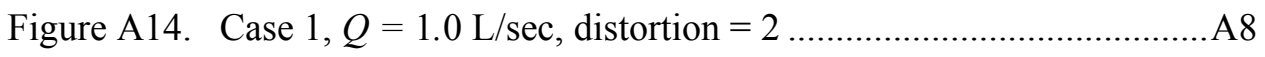

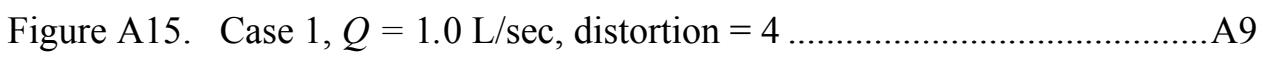

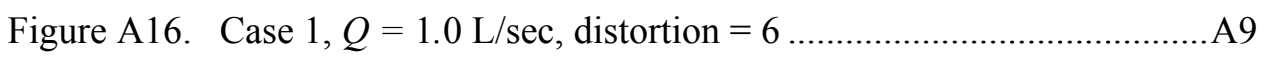

Figure A17. Case $1, Q=1.0 \mathrm{~L} / \mathrm{sec}$, prototype vs. distortion $=2 \ldots \ldots \ldots \ldots \ldots \ldots . . . \mathrm{A} 10$

Figure A18. Case $1, Q=1.0 \mathrm{~L} / \mathrm{sec}$, prototype vs. distortion $=4 \ldots \ldots \ldots \ldots \ldots \ldots$. A 10

Figure A19. Case $1, Q=1.0 \mathrm{~L} / \mathrm{sec}$, prototype vs. distortion $=6 \ldots \ldots \ldots \ldots \ldots \ldots . . . . . . . . .11$

Figure A20. Case $1, Q=1.0 \mathrm{~L} / \mathrm{sec}$, prototype minus distortion $=2$..............A11

Figure A21. Case $1, Q=1.0 \mathrm{~L} / \mathrm{sec}$, prototype minus distortion $=4 \ldots \ldots \ldots \ldots . . \mathrm{A} 12$

Figure A22. Case $1, Q=1.0 \mathrm{~L} / \mathrm{sec}$, prototype minus distortion $=6 \ldots \ldots \ldots \ldots . . . \mathrm{A} 12$

Figure A23. Case $1, Q=1.0 \mathrm{~L} / \mathrm{sec}$, ratios between prototype and distortions 2,4 ,and 6

Figure A24. Case $1, Q=1.0 \mathrm{~L} / \mathrm{sec}$, ratios between prototype and distortions 2 , 4 ,and 6

Figure A25. Case 1, $Q=0.75 \mathrm{~L} / \mathrm{sec}$, prototype..........................................A14

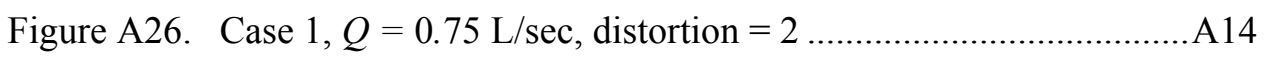

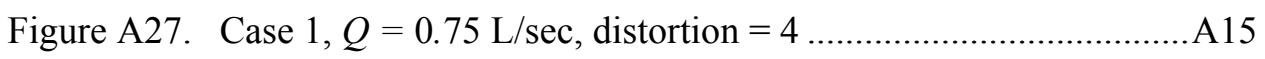

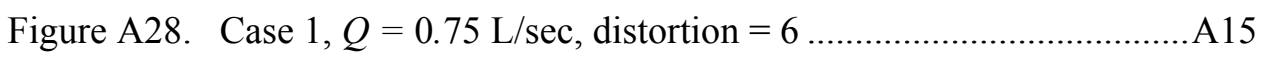

Figure A29. Case $1, Q=0.75 \mathrm{~L} / \mathrm{sec}$, prototype vs. distortion $=2 \ldots \ldots \ldots \ldots \ldots . . . \mathrm{A} 16$

Figure A30. Case $1, Q=0.75 \mathrm{~L} / \mathrm{sec}$, prototype vs. distortion $=4 \ldots \ldots \ldots \ldots \ldots . . . \mathrm{A} 16$

Figure A31. Case $1, Q=0.75 \mathrm{~L} / \mathrm{sec}$, prototype vs. distortion $=6 \ldots \ldots \ldots \ldots \ldots . . . \mathrm{A} 17$

Figure A32. Case $1, Q=0.75 \mathrm{~L} / \mathrm{sec}$, prototype minus distortion $=2 \ldots \ldots \ldots \ldots$... 17

Figure A33. Case $1, Q=0.75 \mathrm{~L} / \mathrm{sec}$, prototype minus distortion $=4 \ldots \ldots \ldots \ldots$. A 18 
Figure A34. Case $1, Q=0.75 \mathrm{~L} / \mathrm{sec}$, prototype minus distortion $=6$

Figure A35. Case $1, Q=0.75 \mathrm{~L} / \mathrm{sec}$, ratios between prototype and distortions 2,4 , and 6

Figure A36. Case $1, Q=0.75 \mathrm{~L} / \mathrm{sec}$, ratios between prototype and distortions 2, 4, and 6 .

Figure B1. Case 2, $Q=1.5 \mathrm{~L} / \mathrm{sec}$, prototype.............................................. B2

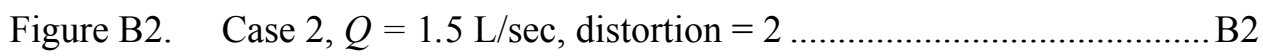

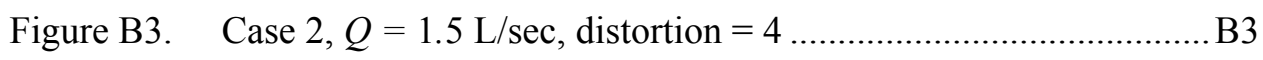

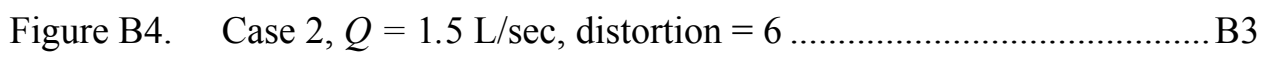

Figure B5. Case $2, Q=1.5 \mathrm{~L} / \mathrm{sec}$, prototype vs. distortion $=2 \ldots \ldots \ldots \ldots \ldots \ldots . . . \mathrm{B} 4$

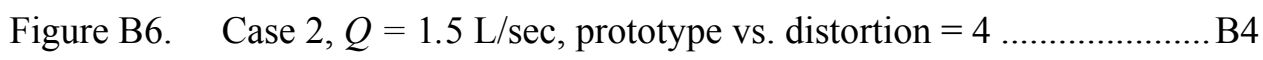

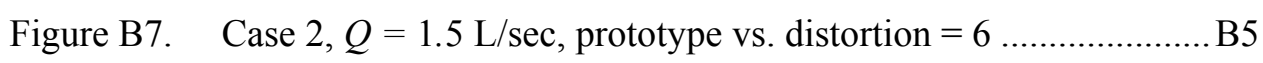

Figure B8. Case $2, Q=1.5 \mathrm{~L} / \mathrm{sec}$, prototype minus distortion $=2 \ldots \ldots \ldots \ldots \ldots . . \mathrm{B} 5$

Figure B9. Case 2, $Q=1.5 \mathrm{~L} / \mathrm{sec}$, prototype minus distortion $=4 \ldots \ldots \ldots \ldots \ldots . . \mathrm{B} 6$

Figure B10. Case 2, $Q=1.5 \mathrm{~L} / \mathrm{sec}$, prototype minus distortion $=6 \ldots \ldots \ldots \ldots \ldots . . . \mathrm{B} 6$

Figure B11. Case $2, Q=1.5 \mathrm{~L} / \mathrm{sec}$, ratios between prototype and distortions 2,4 , and 6 .....

Figure B12. Case $2, Q=1.5 \mathrm{~L} / \mathrm{sec}$, ratios between prototype and distortions 2,4 , and 6 .....

Figure B13. Case 2, $Q=1.0 \mathrm{~L} / \mathrm{sec}$, prototype.............................................. 8

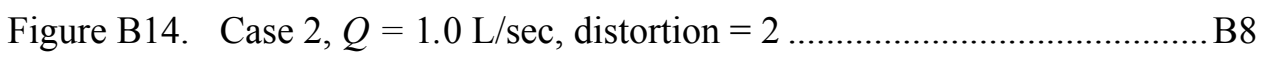

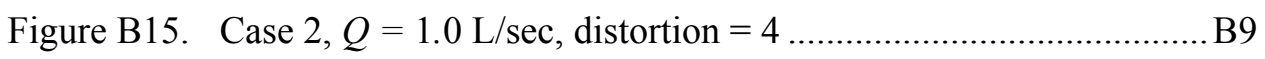

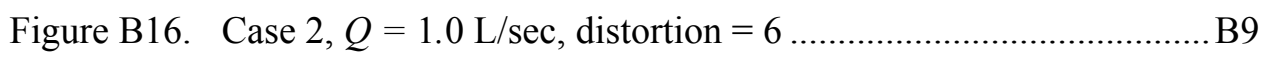

Figure B17. Case $2, Q=1.0 \mathrm{~L} / \mathrm{sec}$, prototype vs. distortion $=2 \ldots \ldots \ldots \ldots \ldots \ldots . . \mathrm{B} 10$

Figure B18. Case $2, Q=1.0 \mathrm{~L} / \mathrm{sec}$, prototype vs. distortion $=4 \ldots \ldots \ldots \ldots \ldots \ldots . . \mathrm{B} 10$

Figure B19. Case 2, $Q=1.0 \mathrm{~L} / \mathrm{sec}$, prototype vs. distortion $=6 \ldots \ldots \ldots \ldots \ldots \ldots . . \mathrm{B} 11$

Figure B20. Case 2, $Q=1.0 \mathrm{~L} / \mathrm{sec}$, prototype minus distortion $=2 \ldots \ldots \ldots \ldots . . \mathrm{B} 11$

Figure B21. Case $2, Q=1.0 \mathrm{~L} / \mathrm{sec}$, prototype minus distortion $=4 \ldots \ldots \ldots \ldots . \mathrm{B} 12$

Figure B22. Case $2, Q=1.0 \mathrm{~L} / \mathrm{sec}$, prototype minus distortion $=6 \ldots \ldots \ldots \ldots . \mathrm{B} 12$

Figure B23. Case $2, Q=1.0 \mathrm{~L} / \mathrm{sec}$, ratios between prototype and distortions 2,4 , and 6

Figure B24. Case $2, Q=1.0 \mathrm{~L} / \mathrm{sec}$, ratios between prototype and distortions 2,4 , and 6 . B13

Figure B25. Case 2, $Q=0.75 \mathrm{~L} / \mathrm{sec}$, prototype.......................................... B14

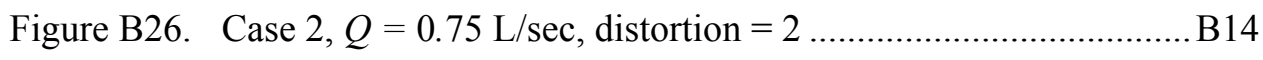

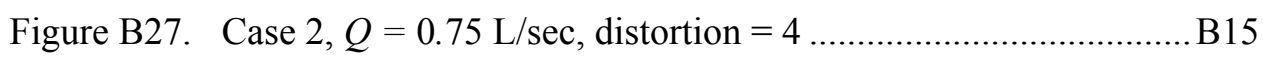




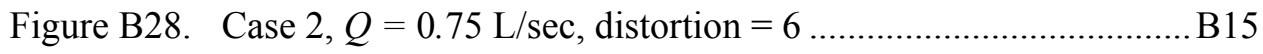

Figure B29. Case 2, $Q=0.75 \mathrm{~L} / \mathrm{sec}$, prototype vs. distortion $=2 \ldots \ldots \ldots \ldots \ldots . . \mathrm{B} 16$

Figure B30. Case 2, $Q=0.75 \mathrm{~L} / \mathrm{sec}$, prototype vs. distortion $=4 \ldots \ldots \ldots \ldots \ldots . . \mathrm{B} 16$

Figure B31. Case $2, Q=0.75 \mathrm{~L} / \mathrm{sec}$, prototype vs. distortion $=6 \ldots \ldots \ldots \ldots \ldots . . \mathrm{B} 17$

Figure B32. Case $2, Q=0.75 \mathrm{~L} / \mathrm{sec}$, prototype minus distortion $=2 \ldots \ldots \ldots \ldots . \mathrm{B} 17$

Figure B33. Case 2, $Q=0.75 \mathrm{~L} / \mathrm{sec}$, prototype minus distortion $=4 \ldots \ldots \ldots \ldots$ B 18

Figure B34. Case $2, Q=0.75 \mathrm{~L} / \mathrm{sec}$, prototype minus distortion $=6 \ldots \ldots \ldots \ldots . \mathrm{B} 18$

Figure B35. Case $2, Q=0.75 \mathrm{~L} / \mathrm{sec}$, ratios between prototype and distortions 2, 4, and 6 .

Figure B36. Case $2, Q=0.75 \mathrm{~L} / \mathrm{sec}$, ratios between prototype and distortions 2,4 , and 6 B19

Figure $\mathrm{C} 1$. Case $3(1 / 3 d), Q=1.5 \mathrm{~L} / \mathrm{sec}$, prototype ..................................... 2

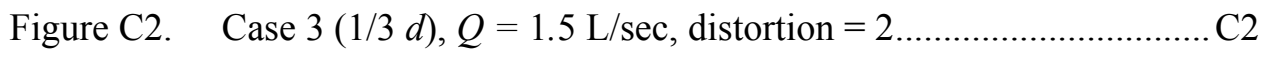

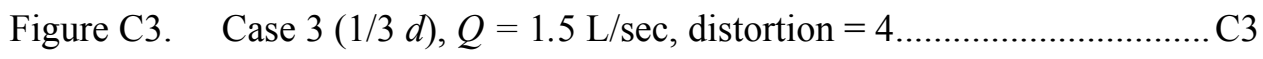

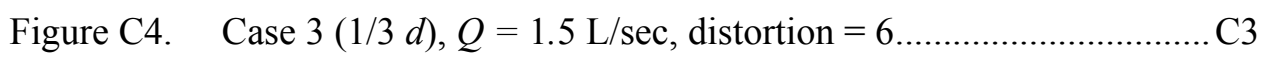

Figure C5. Case $3(1 / 3 d), Q=1.5 \mathrm{~L} / \mathrm{sec}$, prototype vs. distortion $=2 \ldots \ldots \ldots . . \mathrm{C} 4$

Figure C6. Case $3(1 / 3 d), Q=1.5 \mathrm{~L} / \mathrm{sec}$, prototype vs. distortion $=4 \ldots \ldots \ldots . . \mathrm{C} 4$

Figure C7. Case $3(1 / 3 d), Q=1.5 \mathrm{~L} / \mathrm{sec}$, prototype vs. distortion $=6 \ldots \ldots \ldots . . \mathrm{C} 5$

Figure C8. Case $3(1 / 3 d), Q=1.5 \mathrm{~L} / \mathrm{sec}$, prototype minus distortion $=2$

Figure C9. Case $3(1 / 3 d), Q=1.5 \mathrm{~L} / \mathrm{sec}$, prototype minus distortion $=4$

Figure C10. Case $3(1 / 3 d), Q=1.5 \mathrm{~L} / \mathrm{sec}$, prototype minus distortion $=6$

Figure C11. Case $3(1 / 3 d), Q=1.5 \mathrm{~L} / \mathrm{sec}$, ratios between prototype and distortions 2,4 , and 6

Figure C12. Case $3(1 / 3 d), Q=1.5 \mathrm{~L} / \mathrm{sec}$, ratios between prototype and distortions 2,4 , and 6

Figure C13. Case $3(2 / 3 d), Q=1.5 \mathrm{~L} / \mathrm{sec}$, prototype..................................... 8

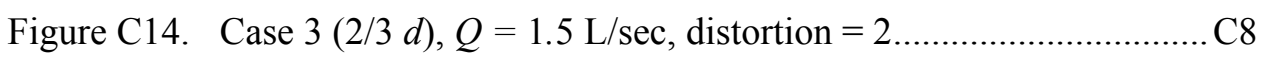

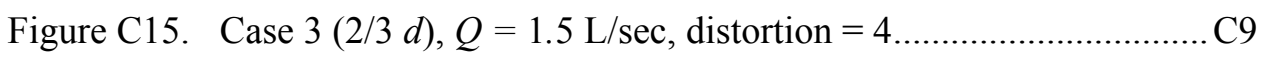

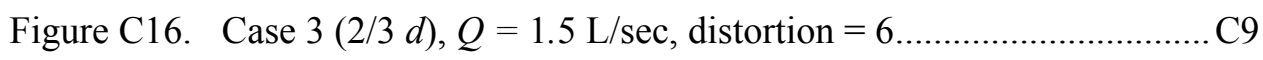

Figure C17. Case $3(2 / 3 d), Q=1.5 \mathrm{~L} / \mathrm{sec}$, prototype vs. distortion $=2 \ldots \ldots \ldots . \mathrm{C} 10$

Figure C18. Case $3(2 / 3 d), Q=1.5 \mathrm{~L} / \mathrm{sec}$, prototype vs. distortion $=4 \ldots \ldots \ldots . \mathrm{C} 10$

Figure $\mathrm{C} 19$. Case $3(2 / 3 d), Q=1.5 \mathrm{~L} / \mathrm{sec}$, prototype vs. distortion $=6 \ldots \ldots \ldots \mathrm{C} 11$

Figure C20. Case $3(2 / 3 d), Q=1.5 \mathrm{~L} / \mathrm{sec}$, prototype minus distortion $=2$ 
Figure C21. Case $3(2 / 3 d), Q=1.5 \mathrm{~L} / \mathrm{sec}$, prototype minus distortion $=4$

Figure C22. Case $3(2 / 3 d), Q=1.5 \mathrm{~L} / \mathrm{sec}$, prototype minus distortion $=6$

Figure C23. Case $3(2 / 3 d), Q=1.5 \mathrm{~L} / \mathrm{sec}$, ratios between prototype and distortions 2,4 , and 6

Figure C24. Case $3(2 / 3 d), Q=1.5 \mathrm{~L} / \mathrm{sec}$, ratios between prototype and distortions 2,4 , and 6

Figure C25. Case $3(1 / 3 d), Q=1.0 \mathrm{~L} / \mathrm{sec}$, prototype.

Figure C26. Case $3(1 / 3 d), Q=1.0 \mathrm{~L} / \mathrm{sec}$, distortion $=2$

Figure C27. Case $3(1 / 3 d), Q=1.0 \mathrm{~L} / \mathrm{sec}$, distortion $=4$

Figure C28. Case $3(1 / 3 d), Q=1.0 \mathrm{~L} / \mathrm{sec}$, distortion $=6$

Figure C29. Case $3(1 / 3 d), Q=1.0 \mathrm{~L} / \mathrm{sec}$, prototype vs. distortion $=2 \ldots \ldots \ldots \mathrm{C} 16$

Figure $\mathrm{C} 30$. Case $3(1 / 3 d), Q=1.0 \mathrm{~L} / \mathrm{sec}$, prototype vs. distortion $=4 \ldots \ldots \ldots \mathrm{C} 16$

Figure $\mathrm{C} 31$. Case $3(1 / 3 d), Q=1.0 \mathrm{~L} / \mathrm{sec}$, prototype vs. distortion $=6 \ldots \ldots \ldots \mathrm{C} 17$

Figure C32. Case $3(1 / 3 d), Q=1.0 \mathrm{~L} / \mathrm{sec}$, prototype minus distortion $=2$

Figure C33. Case $3(1 / 3 d), Q=1.0 \mathrm{~L} / \mathrm{sec}$, prototype minus distortion $=4$

Figure C34. Case $3(1 / 3 d), Q=1.0 \mathrm{~L} / \mathrm{sec}$, prototype minus distortion $=6$

Figure C35. Case $3(1 / 3 d), Q=1.0 \mathrm{~L} / \mathrm{sec}$, ratios between prototype and distortions 2,4 , and 6

Figure C36. Case $3(1 / 3 d), Q=1.0 \mathrm{~L} / \mathrm{sec}$, ratios between prototype and distortions 2,4 , and 6

Figure C37. Case $3(2 / 3 d), Q=1.0 \mathrm{~L} / \mathrm{sec}$, prototype.

Figure C38. Case $3(2 / 3 d), Q=1.0 \mathrm{~L} / \mathrm{sec}$, distortion $=2$.

Figure C39. Case $3(2 / 3 d), Q=1.0 \mathrm{~L} / \mathrm{sec}$, distortion $=4$

Figure C40. Case $3(2 / 3 d), Q=1.0 \mathrm{~L} / \mathrm{sec}$, distortion $=6$

Figure C41. Case $3(2 / 3 d), Q=1.0 \mathrm{~L} / \mathrm{sec}$, prototype vs. distortion $=2 \ldots \ldots \ldots \mathrm{C} 22$

Figure $\mathrm{C} 42$. Case $3(2 / 3 d), Q=1.0 \mathrm{~L} / \mathrm{sec}$, prototype vs. distortion $=4 \ldots \ldots \ldots \mathrm{C} 22$

Figure C43. Case $3(2 / 3 d), Q=1.0 \mathrm{~L} / \mathrm{sec}$, prototype vs. distortion $=6 \ldots \ldots \ldots \mathrm{C} 23$

Figure C44. Case $3(2 / 3 d), Q=1.0 \mathrm{~L} / \mathrm{sec}$, prototype minus distortion $=2$

Figure C45. Case $3(2 / 3 d), Q=1.0 \mathrm{~L} / \mathrm{sec}$, prototype minus distortion $=4$

Figure C46. Case $3(2 / 3 d), Q=1.0 \mathrm{~L} / \mathrm{sec}$, prototype minus distortion $=6$ 
Figure C47. Case $3(2 / 3 d), Q=1.0 \mathrm{~L} / \mathrm{sec}$, ratios between prototype and distortions 2,4 , and 6

Figure C48. Case $3(2 / 3 d), Q=1.0 \mathrm{~L} / \mathrm{sec}$, ratios between prototype and distortions 2,4 , and 6

\section{List of Tables}

Table 1. Commerce at Port of Anchorage in 2001 ........................................2

Table 2. Scale Ratios for Large-Area Model ...................................................11

Table 3. Scale Ratios for Small-Area Model ..................................................17

Table 4. Distorted Model Froude Scales.........................................................39

Table 5. Distortion Experiment Scale Factors and Parameters........................43

Table 6. Measurement Geometry of Vertical Edge Tests ...............................43

Table 7. Distortion in Vertical Step Experiment............................................64

Table 8. Measurement Geometry of Case 4 Vertical Step Experiment ..........64

Table 9. Scale Ratios for Dredge Transition Model ......................................68

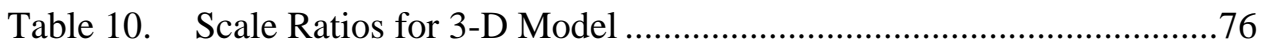




\section{Conversion Factors, Non-SI to SI Units of Measurement}

Non-SI units of measurement used in this report can be converted to SI units as follows:

\begin{tabular}{||l|l|l||}
\hline Multiply & By & To Obtain \\
\hline \hline cubic yards & 0.7645549 & cubic meters \\
\hline feet & 0.3048 & meters \\
\hline inches & 2.54 & centimeters \\
\hline miles (U.S. statute) & 1.609347 & kilometers \\
\hline square miles & 2.58999 & square kilometers \\
\hline
\end{tabular}




\section{Preface}

This technical report describes experiments and flow table simulations of Cook Inlet, AK, conducted by the U.S. Army Engineer Research and Development Center (ERDC), Coastal and Hydraulics Laboratory (CHL), Vicksburg, MS, for the U.S. Army Engineer District, Alaska. The study had two primary purposes: (a) to evaluate in a physical model large-scale complex flow patterns in upper Cook Inlet during maximum ebb and flood tides, and (b) to investigate potential scale effects associated with large-scale, three-dimensional flow turbulence when simulated in geometrically distorted physical models. Initial funding authority was provided by the Alaska District to CHL 21 March 2002 , and a review draft of this report was submitted to the Alaska District 6 January 2003. The Coastal Inlets Research Program (CIRP) provided partial support for this study and report preparation.

Study oversight and review were provided for the sponsoring Alaska District by Messrs. Kenneth J. Eisses and Merlin D. Peterson, Alaska District. Direct participation by the Alaska District engineers in the modeling efforts occurred during two site visits to Vicksburg, MS, in May and November of 2002. Bathymetric data of Cook Inlet was provided to CHL by Mr. Peterson. Ms. Layla M. Raad, CHL, added bathymetry from other sources to create the vertically referenced data set used for the three-dimensional Cook Inlet flow table model.

The physical model study was conducted by Dr. Steven A. Hughes, CHL. Mr. Gian-Marco Pizzo, contractor, operated the flow table, obtained the turbulence measurements, processed the results, and authored portions of the report. Mr. John E. Gullett, ERDC Department of Public Works (DPW), constructed all the flow table models and other study components under the direct supervison of the Model Shop Chief, Mr. Jim T. Schultz.

This study was conducted during the period March 2002 through December 2002 under the general supervision of Mr. Thomas W. Richardson, Director, and Dr. William D. Martin, Deputy Director, respectively, CHL. Direct supervision, valuable advice, and insightful review were provided by Mr. Dennis G. Markle, Chief, Coastal Harbors and Structures Branch. Dr. Nicholas C. Kraus was CIRP Program Manager.

COL James R. Rowan, EN, was Commander and Executive Director of ERDC. Dr. James R. Houston was Director. 


\section{Introduction}

The Port of Anchorage, AK, is located on the upper reach of Cook Inlet as shown in Figure 1. The port began operations in 1961, and since then...

...has expanded to a five-berth terminal providing facilities for the movement of containerized freight, iron and steel products, wood products, bulk petroleum and cement. Anchorage is served regularly by two major carriers, which bring four to five ships weekly from the Pacific Northwest. Petroleum tankers supply jet fuel for airport operations, barges on-load petroleum products for western Alaska and ships from Japan and Korea call frequently transporting pipe, drilling mud, construction materials and automobiles. (From the city of Anchorage Web page, http://www.ci.anchorage.ak.us/ homepage/index.cfm.)

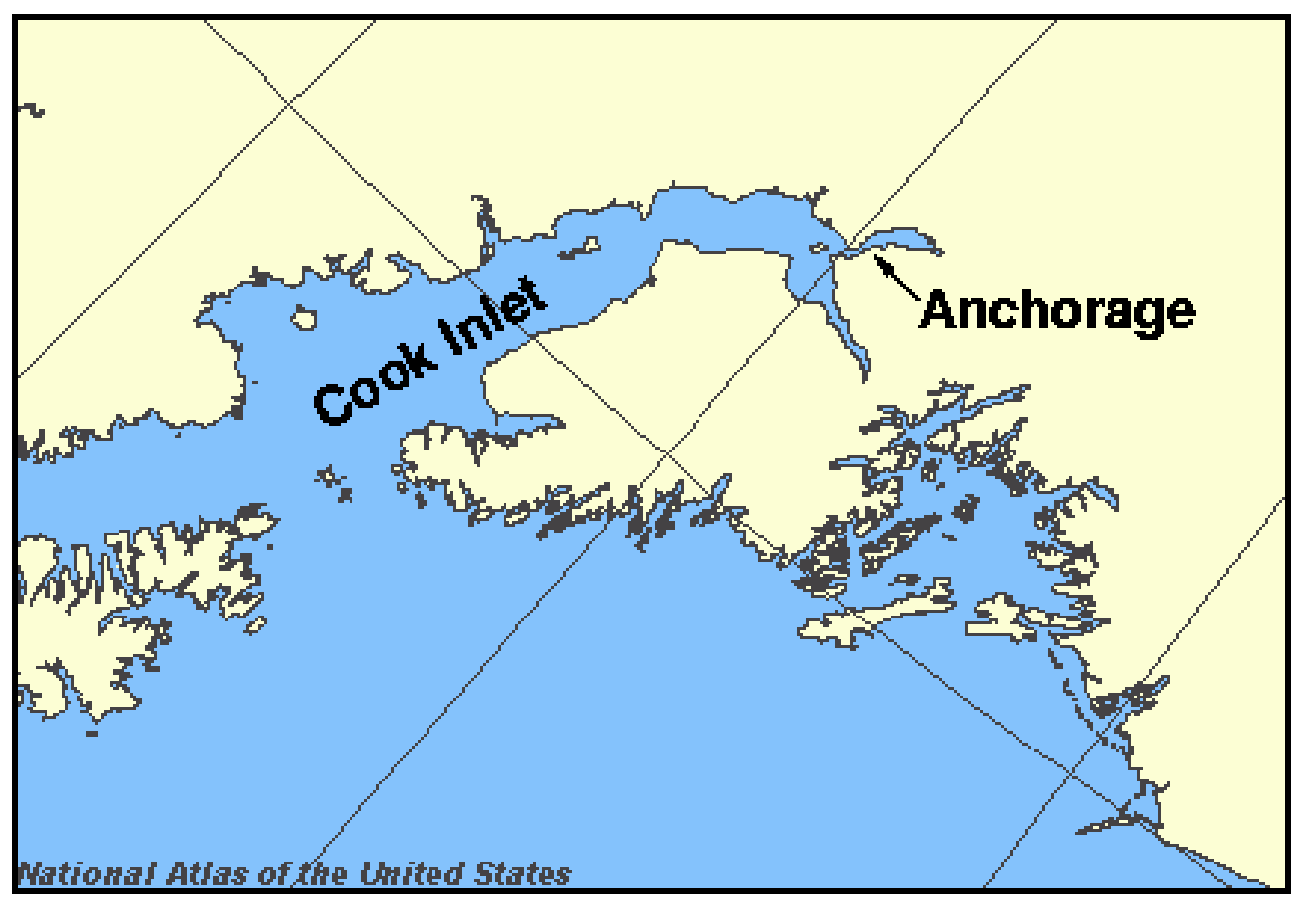

Figure 1. Cook Inlet, AK, location map 
Table 1 gives a breakdown of the almost 3 million tons of shipping handled at the Port of Anchorage in the year 2001.

\begin{tabular}{|l|l|}
\hline $\begin{array}{l}\text { Table } \mathbf{1} \\
\text { Commerce at Port of Anchorage in } \mathbf{2 0 0 1} \text { (City of Anchorage Web } \\
\text { Site) }\end{array}$ \\
\hline Commodity & Tons \\
\hline Freight, NOS & 1,435 \\
\hline Cement, Bulk & 123,065 \\
\hline Iron/Steel & 2,495 \\
\hline Petroleum, NOS & 3,473 \\
\hline Vans/Containers & $1,640,390$ \\
\hline Petroleum, Bulk & $1,203,471$ \\
\hline
\end{tabular}

The port serves 80 percent of Alaska's populated area by rail, road, and air connections, and it handles more than 90 percent of all consumer goods sold in the region. All of the refined petroleum products from Alaska's largest refinery in Fairbanks pass through the Port of Anchorage. Figure 2 shows an aerial view of the port's berthing area and shore infrastructure.

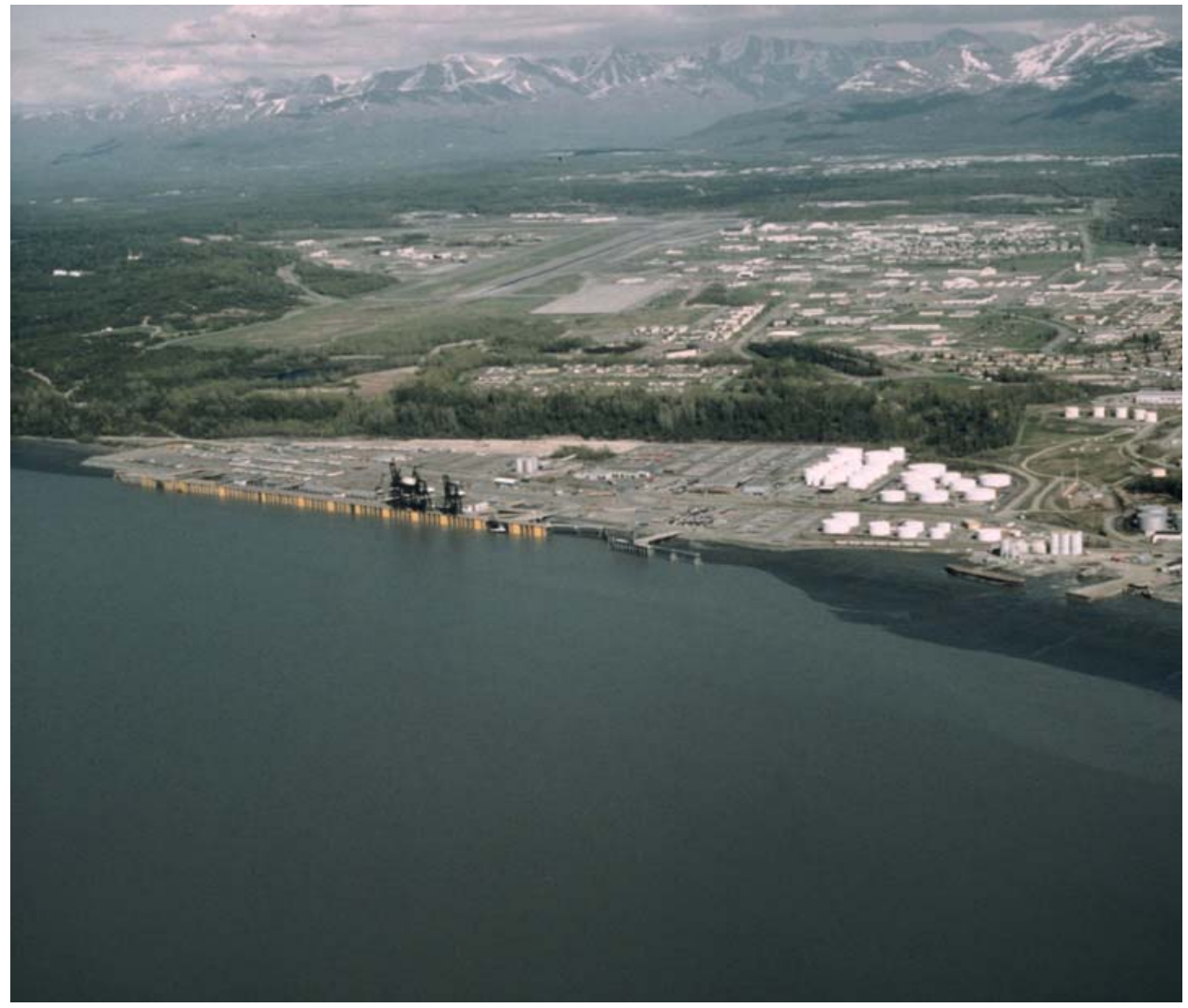

Figure 2. Port of Anchorage, looking south 


\section{Problem Background}

Shoaling at the Port of Anchorage during the summer months has required annual dredging that averages between 152,911 and 305,821.9 cu m/year (200,000 and 400,000 cu yd/year) ${ }^{1}$ with occasional larger deposition quantities of between $611,643.9$ and 764,554.9 $\mathrm{cu} \mathrm{m}(800,000$ and 1,000,000 cu yd). The deposited material is mostly fine silts. Because there is no way to predict the larger deposition episodes, the Alaska District budgets for typical dredging cycles, and then has to perform emergency dredging during years when greater deposition occurs. Emergency dredging involves substantial mobilization costs, which must be covered by additional appropriation and by shifting funds within the operations and maintenance budget.

The Alaska District is undertaking studies to examine several questions related to the operation and maintenance of the Port of Anchorage. In particular, the District is interested in:

a. Determining the source(s) of fine sediment being deposited in Anchorage Harbor.

b. Devising some means of predicting in advance when increased sediment deposition is expected so adequate funds can be allocated a priori.

c. Examining potential harbor modifications that might decrease deposition within the harbor.

A key component to answering these questions is better understanding of the hydrodynamic regime. Tidal flows with maximum currents above $2 \mathrm{~m} / \mathrm{sec}$ (6.56 ft.sec) (4 knots) result from the 10-m (33-ft) tide range. These currents are capable of transporting huge quantities of fine sediment eroded from the extensive tidal flats upstream and downstream of Anchorage. Prominent headlands such as Point MacKenzie, Point Woronzof, and Cairn Point cause separation of the tidal flow along with generation of gyres and formation of reduced flow areas in the lee of the headlands. The whole process of flow separation, flow entrainment, and lateral shearing of the currents leads to significant flow variations both through the water column and laterally across the inlet. If these three-dimensional (3-D) hydrodynamic processes contribute substantially to shoaling of the Port of Anchorage, then it is critical that simulation tools used to study the problem include 3-D capabilities.

\section{Study Purpose and Components}

The study performed at the U.S. Army Engineer Research and Development Center's (ERDC) Coastal and Hydraulics Laboratory (CHL) had two principal components which are discussed in the following paragraphs.

\section{Flow table physical models}

The first component was to use small-scale physical models to examine the complex flow conditions in upper Cook Inlet. Under steady flow conditions

\footnotetext{
${ }^{1}$ Units of measurement in the text of this report are shown in SI units followed by non-SI units in parenthesis. A table of factors for converting non-SI to SI units of measusrement used in figures and tables is presented on page xiii.
} 
representing various stages of the tide, the model boundaries generate complex patterns that include large-scale turbulent-like features such as gyres and 3-D velocity structures. The flow patterns reveal regions of faster and slower current velocities, and potential sediment deposition areas can be readily identified.

The purpose of the small-scale physical models was to perform an initial investigation of the complex flow regime using an economical tool with the hope of identifying the physical mechanism responsible for shoaling of the Port of Anchorage. An added benefit of the models was the capability to modify physical conditions and immediately observe changes brought about in the system. This allowed potential engineering solutions to be examined rapidly. For example, impacts caused by structures intended to redirect flows could be assessed and optimized using the small-scale models.

\section{Turbulent scale effect in geometrically distorted physical models}

The second component was to examine potential turbulence scale effects that might occur in geometrically distorted physical models. The Alaska District is contemplating construction of a large physical model to simulate tidal flow in Cook Inlet and at the Port of Anchorage. The proposed model would be constructed at considerable expense, and it would be the primary tool for investigating problems and developing solutions for the region centered on the Port of Anchorage. The area encompassed in this model would require that the model horizontal length scale would be different than the vertical length scale. Generally, geometric distortion of tidal flow models is an accepted practice because vertical velocities and accelerations are considered negligible. However, flow patterns in Cook Inlet exhibit regions of flow separation and entrainment, which can produce non-negligible vertical components to the flow. If these vertical flow structures are thought to be important to the shoaling and scour processes that are to be examined in the large-scale physical model, it is crucial to determine potential scale effects that might arise from incorrectly simulating 3-D turbulence in geometrically distorted physical models.

The purpose of this component is to assess the turbulence scale effect that arises in geometrically distorted physical models of tidal flow, and to determine whether this scale effect would adversely impact results obtained from the proposed large-scale model of Cook Inlet.

\section{Study Tasks}

The original scope of work called for four study tasks. A fifth task was added based on observations of the physical models during the first site visit by Alaska District engineers. These tasks are briefly described as follows:

\section{Task 1: Large-area idealized flow model of Cook Inlet}

The objective of this task was to identify and examine in a semiquantitative way the large-scale flow patterns in upper Cook Inlet during maximum ebb and flood tide. 


\section{Task 2: Small-area idealized flow model of Cook Inlet}

The objective of this task was to study in greater detail flow patterns identified in the Task 1 model in terms of potential contribution to sedimentation at the Port of Anchorage. A secondary objective was to collect data to compare between the two small-scale distorted models.

\section{Task 3: Turbulence scale effect in distorted physical models}

The objective of Task 3 was to quantify the impact of turbulent scale effects associated with geometrically distorted physical flow models, and to determine whether a proposed large-scale distorted physical model of Cook Inlet will produce reliable and useful results in regions where flow separation and turbulence are contributing to sedimentation.

\section{Task 4: Effect of sloping transitions on flow hydrodynamics}

The objective of this task was to test the hypothesis that sloping transitions on both ends of the harbor would decrease areas of flow reduction and increase flushing of the harbor. (Presently, the upstream and downstream ends of the dredged region of the Port of Anchorage have vertical sidewalls cut out of hard, non-erodible material.)

\section{Task 5: Large-area 3-D flow model of Cook Inlet}

The objective of this task was to identify and examine in a 3-D flow table model the large-scale flow patterns in upper Cook Inlet during maximum ebb and flood tide.

\section{Report Organization and Content}

The chapters of this report are organized according to the previously listed tasks. Chapter 2 overviews the flow table modeling facility and describes its capabilities and operation. Chapter 3 describes the two idealized Cook Inlet models and summarizes observations from these models. Turbulence scale effects are examined theoretically in Chapter 4, along with an examination of potential scale effects for river bends simulated in geometrically distorted physical models. Flow table experiments to examine potential turbulence scale effects in distorted models are described in Chapter 5 along with results and conclusions. Chapter 6 presents experiments related to potential shoaling impacts caused by lateral boundaries of the dredging area. The 3-D geometrically distorted flow table model of upper Cook Inlet is described in Chapter 7. Finally, all the study tasks are summarized in Chapter 8, and study conclusions are listed and discussed. Complete plotted results from the turbulence scale effect experiments are included in Appendix A, Appendix B, and Appendix C. 


\section{Coastal and Hydraulics Laboratory Flow Table}

\section{Flow Table General Description}

The precision flow table at CHL is used to examine flow problems related to tidal flows in estuaries and currents interacting with inlet jetty structures. The system maintains a constant flow discharge across a horizontal portion of the table through a recirculating system regulated by valves. Water depth is controlled by a downstream adjustable weir. Small-scale models depicting either idealized flow boundaries or portions of actual projects are placed on the glass horizontal test section of the flow table. Complex flow patterns created by the scale model solid boundaries, such as regions of flow separation and turbulence generation, are quantified using a laser Doppler velocimeter (LDV) located beneath the horizontal section of the table. The laser beams pass through the glass bottom and measure two horizontal components of velocity at the vertical elevation where the laser beams intersect. Complex flow patterns can also be visualized using traditional techniques of dye injection, surface tracers, and bottom tracers.

For Corps projects where 3-D flow structures are thought to be significant, the flow situation can be clarified by fabricating a scale model of the actual bathymetry and shore boundaries for use on the flow table. Changes to bathymetry or upstream boundaries are easily simulated, and the impact is immediately observed. Because many complex flow phenomena such as separation and turbulence are reliably reproduced in small-scale physical models, the flow table can be used as a validation tool in conjunction with development of advanced hydrodynamic numerical models that incorporate these features.

\section{Flow Table Details}

The CHL flow table, shown in the photograph of Figure 3 and represented schematically in Figure 4, is approximately the size of a billiards table. Flow of water from the constant head tank (HT) is controlled by a valve, which assures a steady flow rate feeding the upstream basin (IN). Water flows across the horizontal $2.44 \mathrm{~m} \times 1.22 \mathrm{~m}(8 \mathrm{ft} \times 4 \mathrm{ft})$ glass bottom of the flow table and spills over the adjustable-height weir into the catchment tank (OUT), which in turn overflows into the reservoir (RES). The reservior is detached from the flow table to isolate vibrations of the pump as water is recirculated to the head tank. 
The discharge rate onto the flow table is controlled by an adjustable valve (A1) and flow meter.

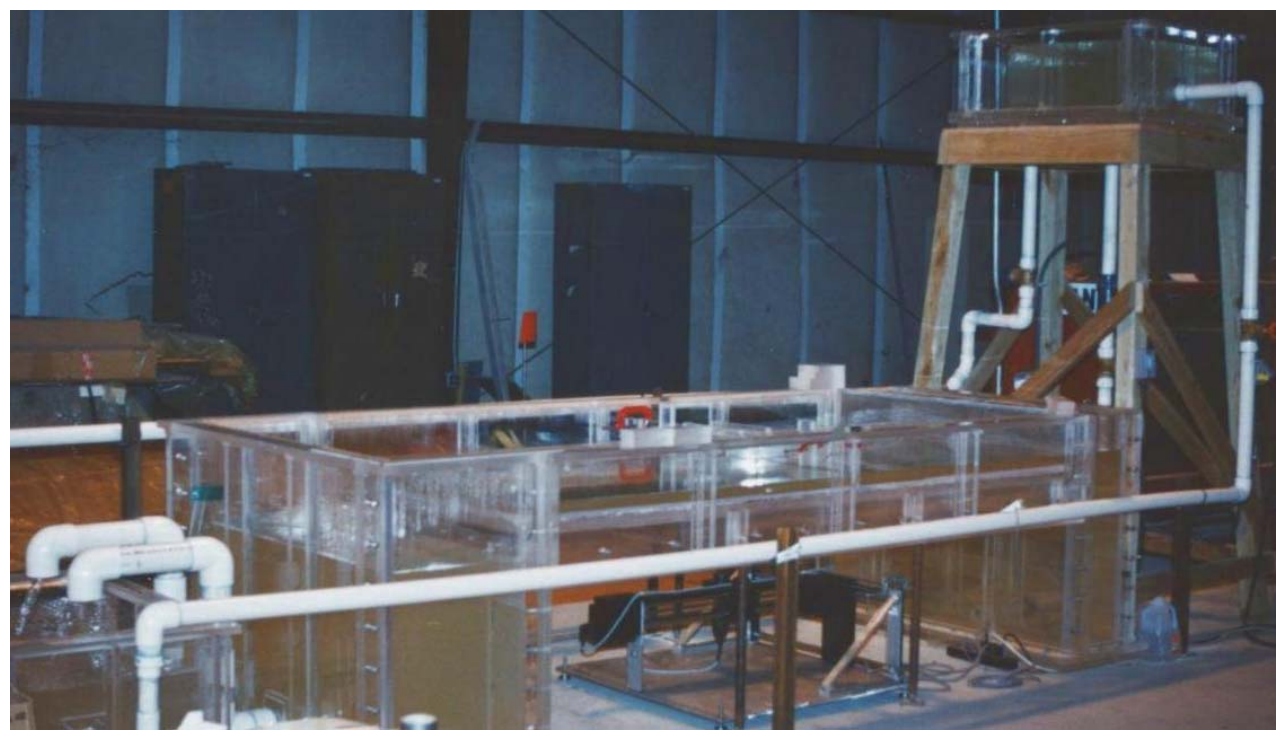

Figure 3. Side view of $\mathrm{CHL}$ flow table

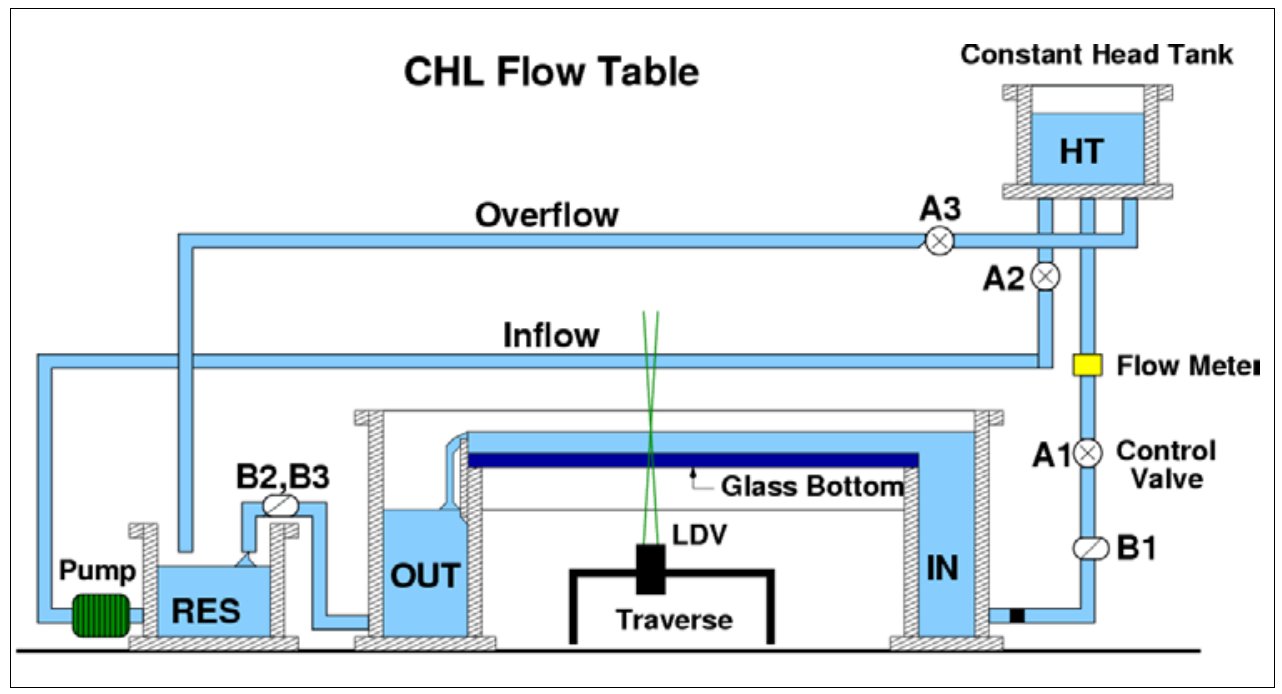

Figure 4. Schematic side view showing flow table components

\section{Flow Measurement System}

Flow velocities on the water table are measured with a two-component laser Doppler fiber-optic probe mounted on a horizontal traversing system beneath the 19-mm-thick glass bottom (Figure 5). Traversing of the LDV probe is computer 
controlled in two horizontal directions allowing automatic recording of velocity at precise, predetermined locations throughout the testing area. Usually, velocity data are collected on a uniformly spaced grid with the probe collecting a time series of instantaneous velocities at each grid point before moving to the next location. The velocity time series in the two orthogonal horizontal directions at each point are averaged to provide two components of the velocity vector.

Sampling rate and duration are adjustable, but typically data are collected at a $100-\mathrm{Hz}$ rate for $10 \mathrm{sec}$ at each point. Because the flow is quasi-steady, the final result is a map of velocity vectors detailing the flow throughout the measurement region.

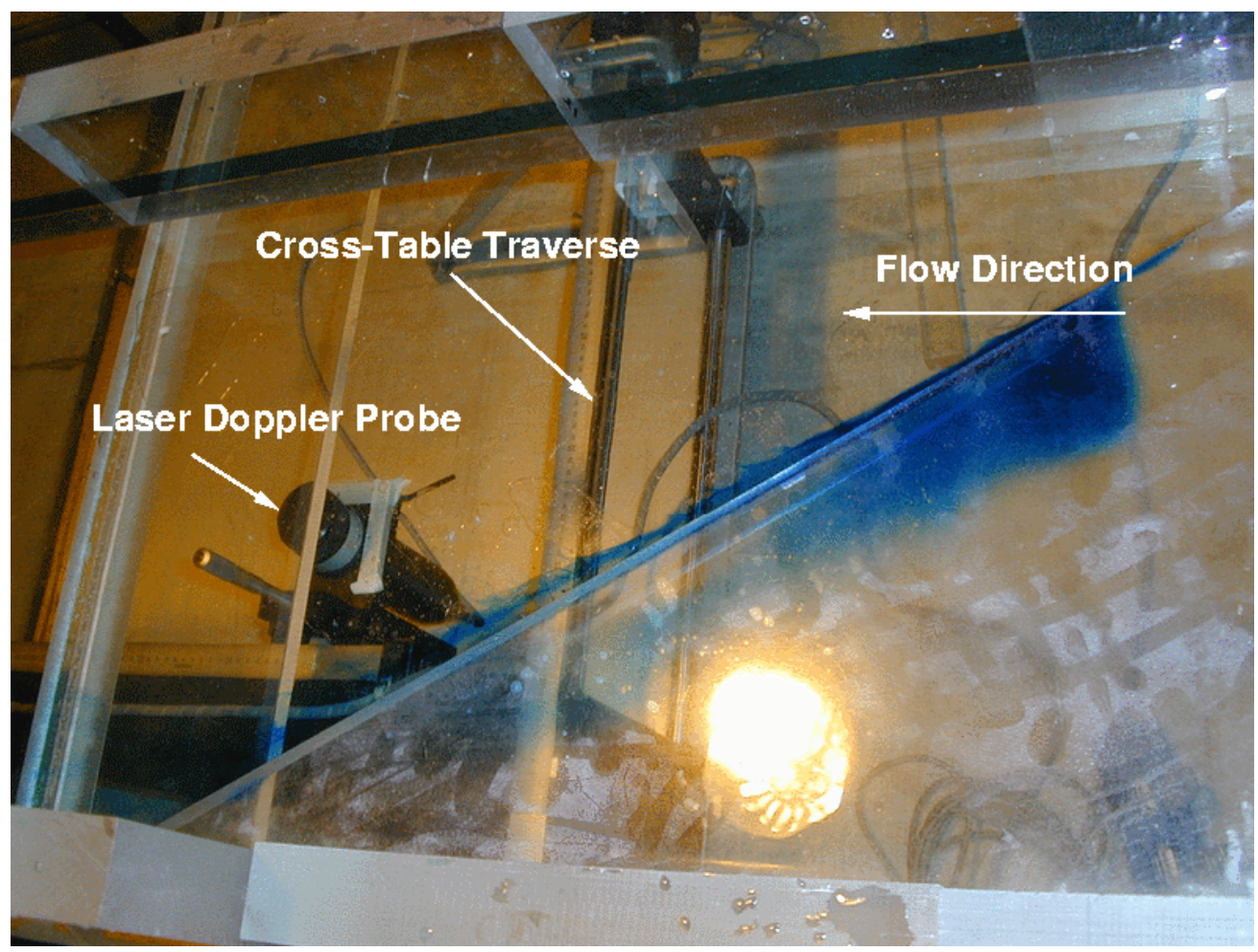

Figure 5. View of traversing system looking down through glass bottom

Velocity measurements using the traversing LDV system require a transparent horizontal bottom so that the four individual laser beams will have equal refraction and converge at a point. Consequently, velocity measurements cannot be made when actual bathymetry is carved into Plexiglas and placed over the horizontal glass bottom because the laser beams would not converge to a point. However, a compromise is possible if the bathymetry is idealized as a series of stepped horizontal surfaces fabricated of Plexiglas. Flow quantification for models incorporating complex bathymetry must be done using surfacepiercing instruments such as LDV probes, microimpellers, or by using time-lapse photography of surface tracers. 


\section{Idealized Cook Inlet Models}

Field observations of flow patterns in upper Cook Inlet, visualized by ice breakup in the spring, had previously indicated that large-scale flow separation occurred at major headland features. The flow separation generated 3-D flow structures, and it was hypothesized that this might be a mechanism responsible for the formation of deep scour holes and sedimentation in the Port of Anchorage.

\section{Overview of Idealized Models}

Two idealized scale models of a portion of upper Cook Inlet, AK, were constructed and tested on the CHL precision flow table. The purpose of these models was to observe and identify the predominant large-scale tidal flow patterns responsible for sediment erosion and deposition in the vicinity of the Port of Anchorage.

These models were termed "idealized" because actual bathymetry in the modeled region was represented by several horizontal surfaces situated at selected depth contours. Transitions between different depths were vertical, and the shoreline was also vertical rather than sloping. So, in essence, the bathymetry had a "terraced" look. There were three reasons for this bathymetry compromise:

a. The models would be inexpensive and easy to build.

$b$. The models could be rapidly constructed based on nautical charts.

c. The horizontal surfaces constructed of clear Plexiglas would allow velocity measurements to be obtained throughout most of the flow regime using the laser Doppler velocimeter.

It was judged that the idealized bathymetry would produce flow patterns similar to models having actual bathymetry, but it was recognized the vertical shoreline and vertical transitions between depths would have some impact on the 3-D flow structure, thus compromising model results.

The second major concession in the idealized models was geometric distortion. Both idealized models had significant geometric distortion with the horizontal scale much larger than the vertical scale. The reason for the distortion was to be able to model a vast horizontal portion of Cook Inlet at the small size of the flow table. In general, smooth steady and unsteady nonoscillatory flow is not compromised by geometric distortion. However, this was not the case for regions of the flow where vertical velocities and accelerations were significant, 
such as in the region of flow separation. The impact of model distortion on the turbulent flow regions was unknown during testing of the idealized models. That question was subsequently examined in a later portion of this study (see Chapters 4 and 5).

Despite the model distortion and idealized bathymetry, the two idealized models produced flow patterns that were visually similar to those observed in the field, and this lent credence to the observations made and hypotheses developed during testing of the idealized models.

The two idealized models were referred to as the large-area model and the small-area model as delineated in the map in Figure 6. The large-area model was

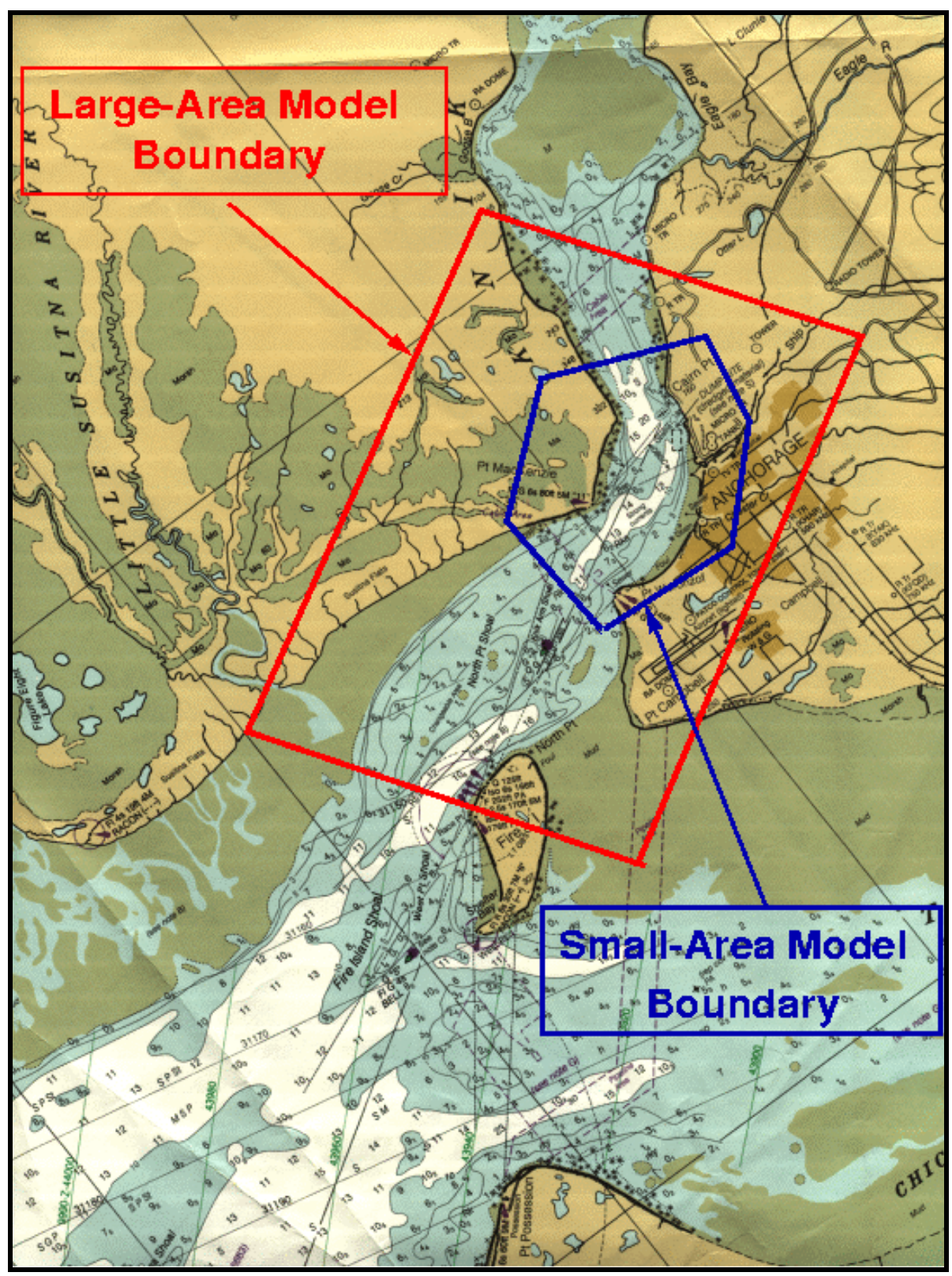

Figure 6. Area coverage of large-area and small-area idealized models 
intended to give a broad overall representation of the flow patterns, whereas the small-area model would permit a closer look at the flow features in the general vicinity of the Port of Anchorage.

\section{Large-Area Model Description}

The seaward boundary of the large-area idealized model reproduced about 10.3 nautical miles (n.m.) on a transect through Fire Island as shown in Figure 6. The shoreward boundary extended a distance of about 5 n.m. upstream of the Port of Anchorage. This area was scaled to fit within the 1.22-m (4-ft) width and 2.44-m (8-ft) length of the CHL flow table. Relevant scale ratios ${ }^{1}$ for the largearea model are listed in Table 2 along with approximate model equivalences.

\begin{tabular}{|c|c|c|}
\hline \multicolumn{3}{|c|}{\begin{tabular}{|l} 
Table 2 \\
Scale Ratios for Large-Area Model
\end{tabular}} \\
\hline Scale & Scale Value & Model Equivalence \\
\hline Horizontal Scale & $N_{X}=15,625$ & $1,300 \mathrm{ft} \approx 1$ in. \\
\hline Vertical Scale & $N_{Z}=480$ & $40 \mathrm{ft}=1 \mathrm{in}$ \\
\hline Velocity Scale & $N_{V}=\sqrt{N_{Z}}=21.9$ & $2.2 \mathrm{~m} / \mathrm{sec}=10 \mathrm{~cm} / \mathrm{sec}$ \\
\hline Discharge Scale & $N_{Q}=N_{X} N_{Z}^{3 / 2}=164,316,767$ & $203,000 \mathrm{cu} \mathrm{m} / \mathrm{sec}=1.24$ liters $/ \mathrm{sec}$ \\
\hline
\end{tabular}

Depths in the model were idealized by a horizontal surface located at an elevation corresponding to depths of el 0 and by the glass bottom of the flow table, which represented el $-60 .^{2}$ Transitions between the two depths were vertical. Figure 7 shows the modeled region as it was situated on the flow table. The red lines represent the shoreline, and the blue lines are el 0.

The ERDC Model Shop converted the shoreline and el 0 contours into appropriate commands for cutting by a computerized router. The components for the idealized model were then cut from blocks of 3.81-cm (1.5-in.-) thick Plexiglas (Figure 8). Figure 9 shows the large-area model during assembly.

The scaled idealized model was operated by placing it on the flow table, turning on the recirculation pump, and adjusting the valves to give the appropriate total discharge. The adjustable downstream weir was used to set the constant water level. Thus, the model simulated steady tidal flow of given total discharge at the selected tide elevation. Simulation of a variable-flow tide hydrograph was not supported at this time. Changing between ebb and flood tide was accomplished by rotating the entire model by $180 \mathrm{deg}$ on the flow table so the water flowed across the model in the opposite direction.

\footnotetext{
${ }^{1}$ Scale ratios are defined as the value of a parameter in the prototype divided by the value in the model. For example, the scale ratio of the characteristic horizontal length $X$ is:

$$
N_{X}=\frac{X_{p}}{X_{m}}=\frac{\text { Value of } \mathrm{X} \text { in Prototype }}{\text { Value of } \mathrm{X} \text { in Model }}
$$

${ }^{2}$ All elevations (el) cited herein are in feet referred to mean lower low water (mllw) datum (to convert feet to meters, multiply number of feet by 0.3048 ).
} 


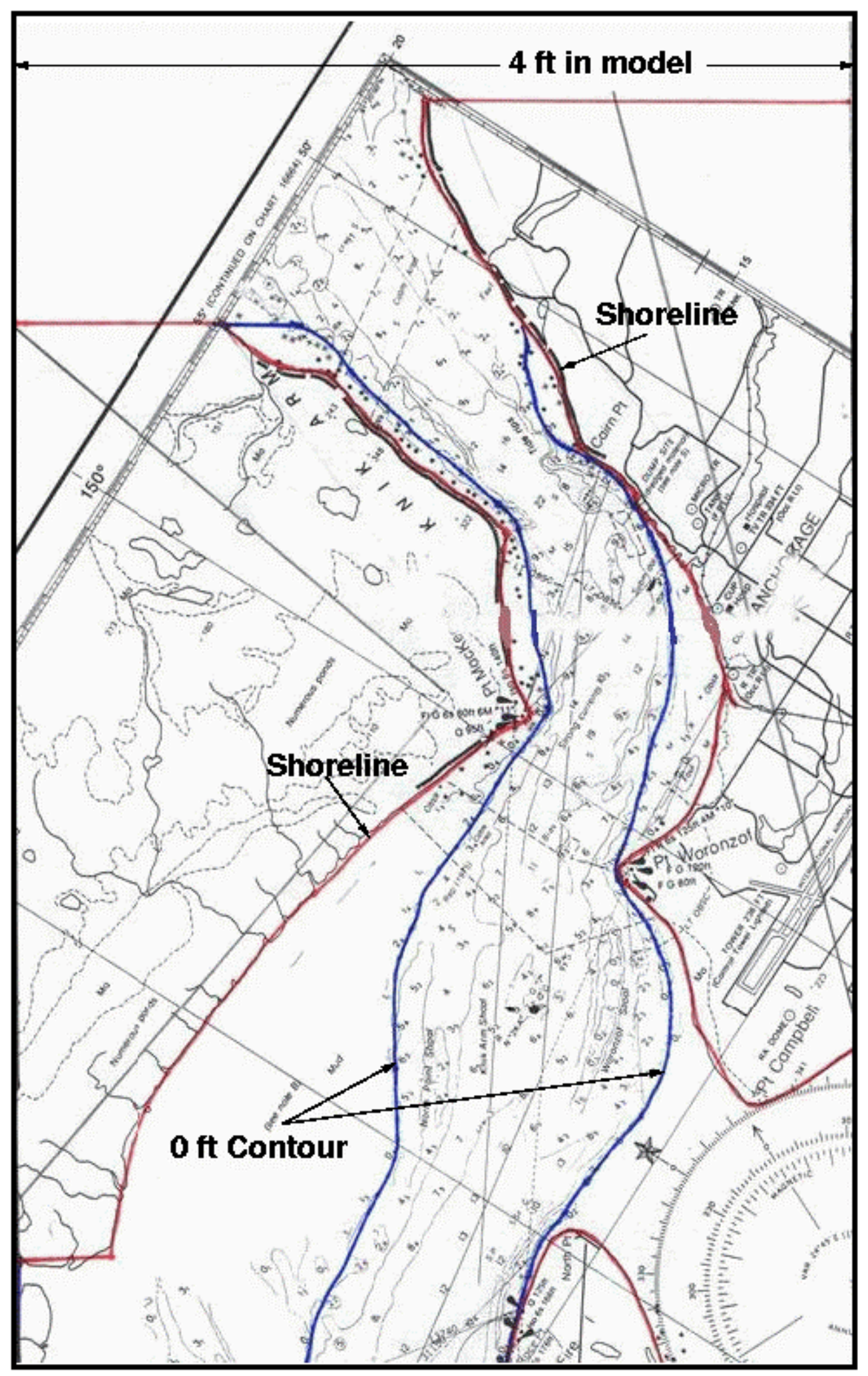

Figure 7 . Idealized bathymetry in large-area model 


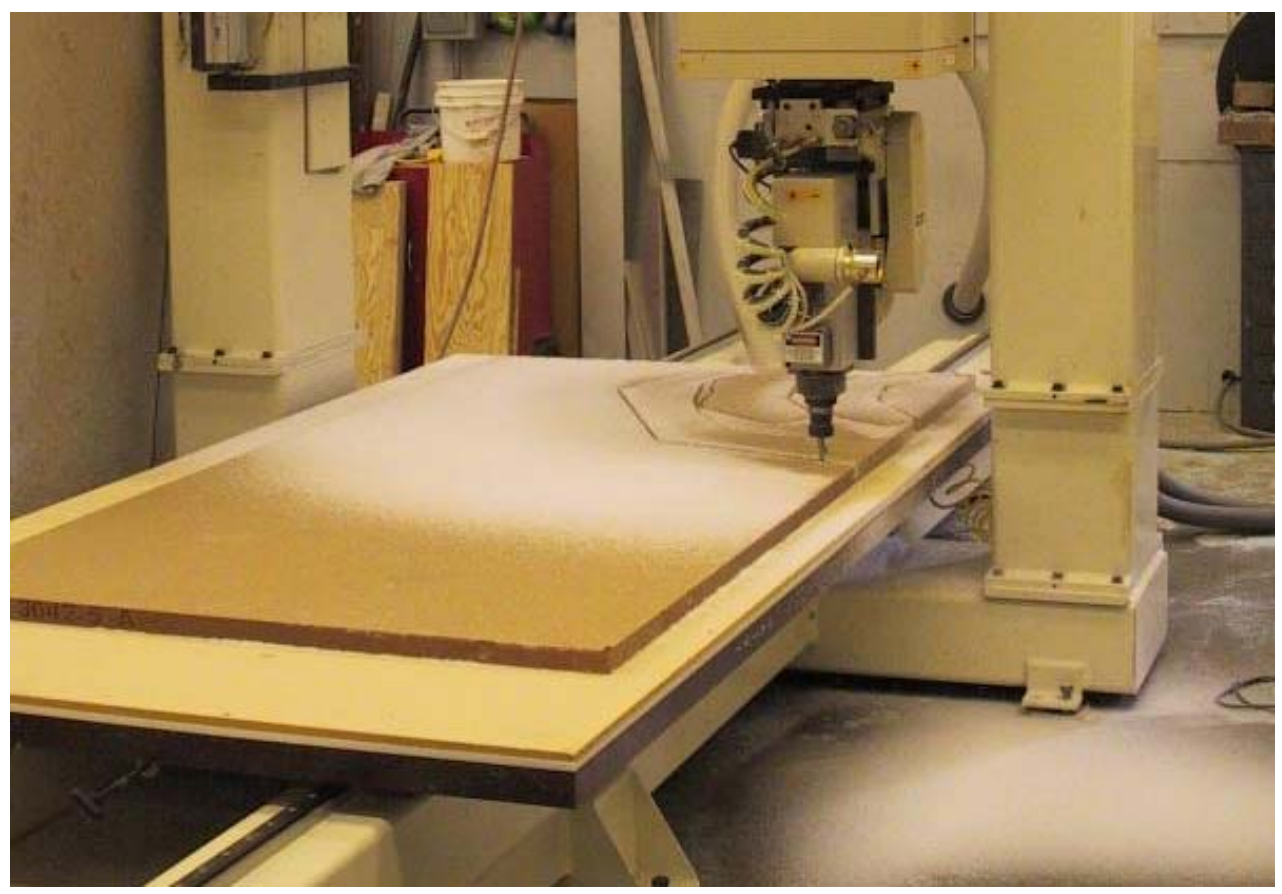

Figure 8. Cutting large-area model pieces with router

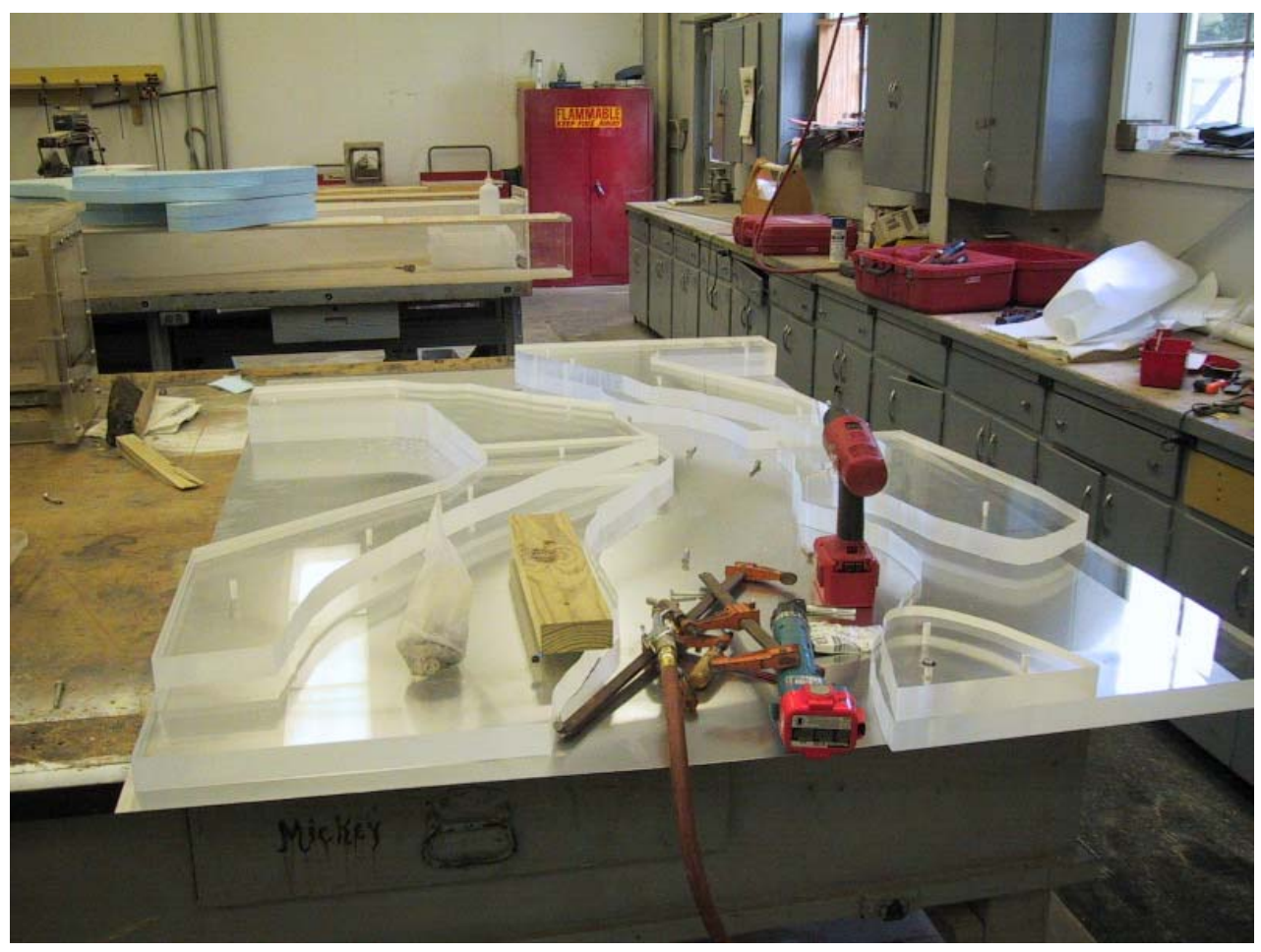

Figure 9. Assembly of large-area model 
Complex flow patterns were visualized using injected dyes and tracers introduced into the upstream flow. The dye revealed the 3-D nature of the turbulence, and it was injected using different sized syringes. One method is to squirt a line of dye across the principle flow direction to observe how it translates downstream. A second method is to continually introduce dye at an upstream point so a path line forms as the dye moves downstream.

Baby powder worked well as a tracer. Quite by accident it was learned that one brand of baby powder had small particle sizes so the flakes floated on the surface, whereas another brand of powder had larger flakes that sank to the bottom before moving downstream.

\section{Large-Area Model Observations}

During the week of 6-10 May 2002, engineers from the Alaska District arrived at ERDC to examine ebb and flood general flow patterns using the idealized flow table models. Testing commenced with the large-area model placed on the flow table to represent the peak flood flow condition. According to the Alaska District engineers, the large-area model

“...appeared to reproduce reasonably well known surface conditions. The most important of these conditions are the gyres/eddies around the Port of Anchorage, Point Woronzof, and Point MacKenzie."

It was also noted that upstream model boundaries influenced the flow patterns downstream. Various solid objects were used to represent boundary changes such as shifting shoals. For example, a lateral movement of Fire Island shoal an equivalent of $914.4 \mathrm{~m}(3,000 \mathrm{ft})$ changed the downstream location of a crosschannel current by about $1,828.8 \mathrm{~m}(6,000 \mathrm{ft})$.

Generally, the flood and ebb surface currents moved as expected with large gyres forming in the lee of Point Woronzof, Point MacKenzie, and Cairn Point. Figure 10 shows the large-area model during flood tide with surface flows visualized by tracers. The closer view offered by Figure 11 shows tracers trapped in the reduced flow area to the lee of Point MacKenzie.

Dye injection indicated significant 3-D flow structure within the large gyres (Figure 12). An unexpected observation was formation of a strong cross-channel current at the bottom during flood tide. This current originated to the north of Point Woronzof and crossed the channel on a diagonal toward Port MacKenzie as illustrated in Figure 13. The mechanism for this cross-channel flow appeared to be flood flow separation at Point MacKenzie, which accelerated the flow at the separation boundary resulting in a local decrease in water-surface elevation. The cross-channel water level differential created a momentum imbalance that was alleviated by mass flowing from the higher side to the lower side at the bottom where resistance to cross-channel flow was least. Although this phenonomenon had not been observed in the field, it appeared to be authenic because the necessary flow separation was known to occur at Point MacKenzie. An important observation was that the stepped contours in the idealized model seemed to prevent vertical mixing between the layers. 


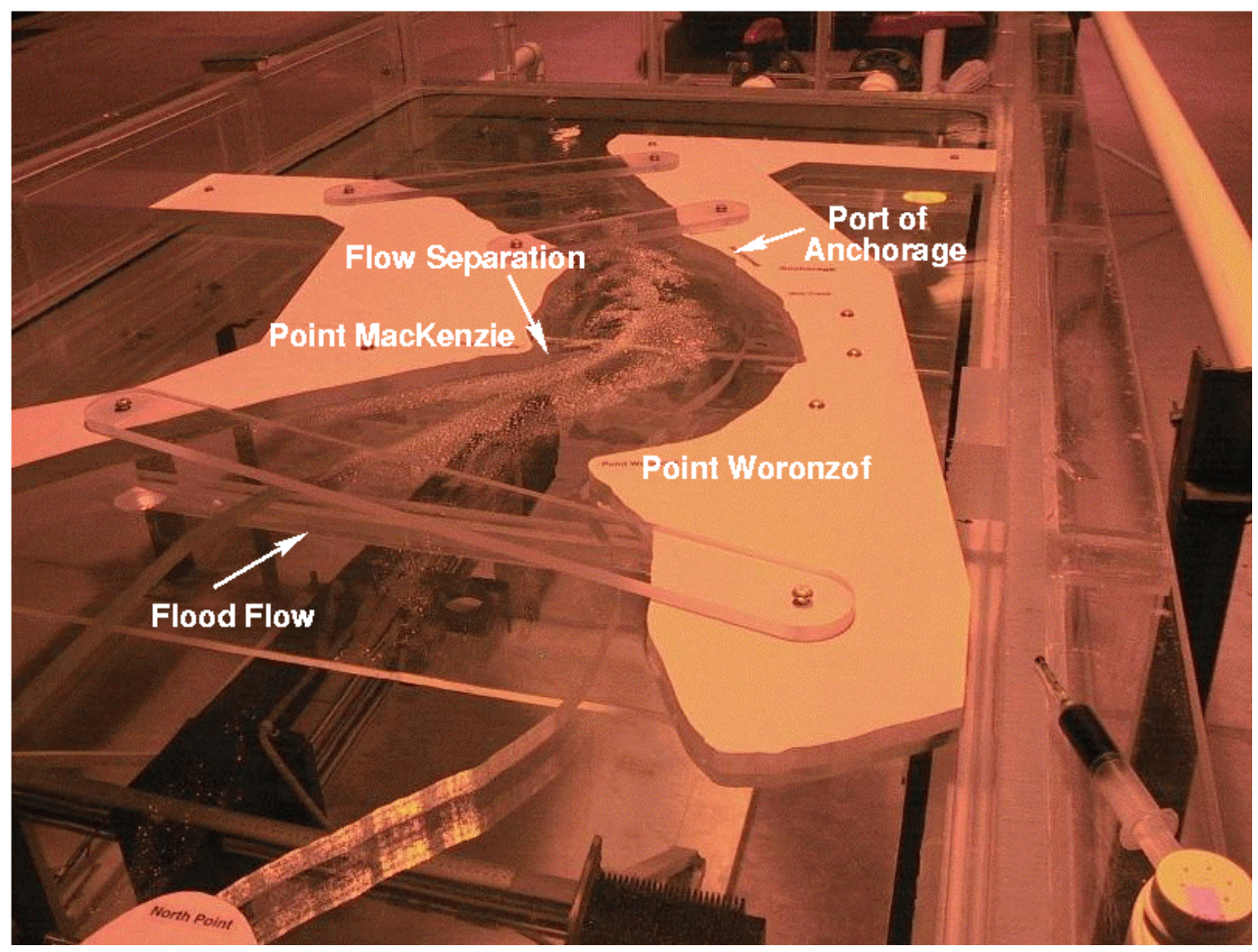

Figure 10. Flood flow in large-area idealized model

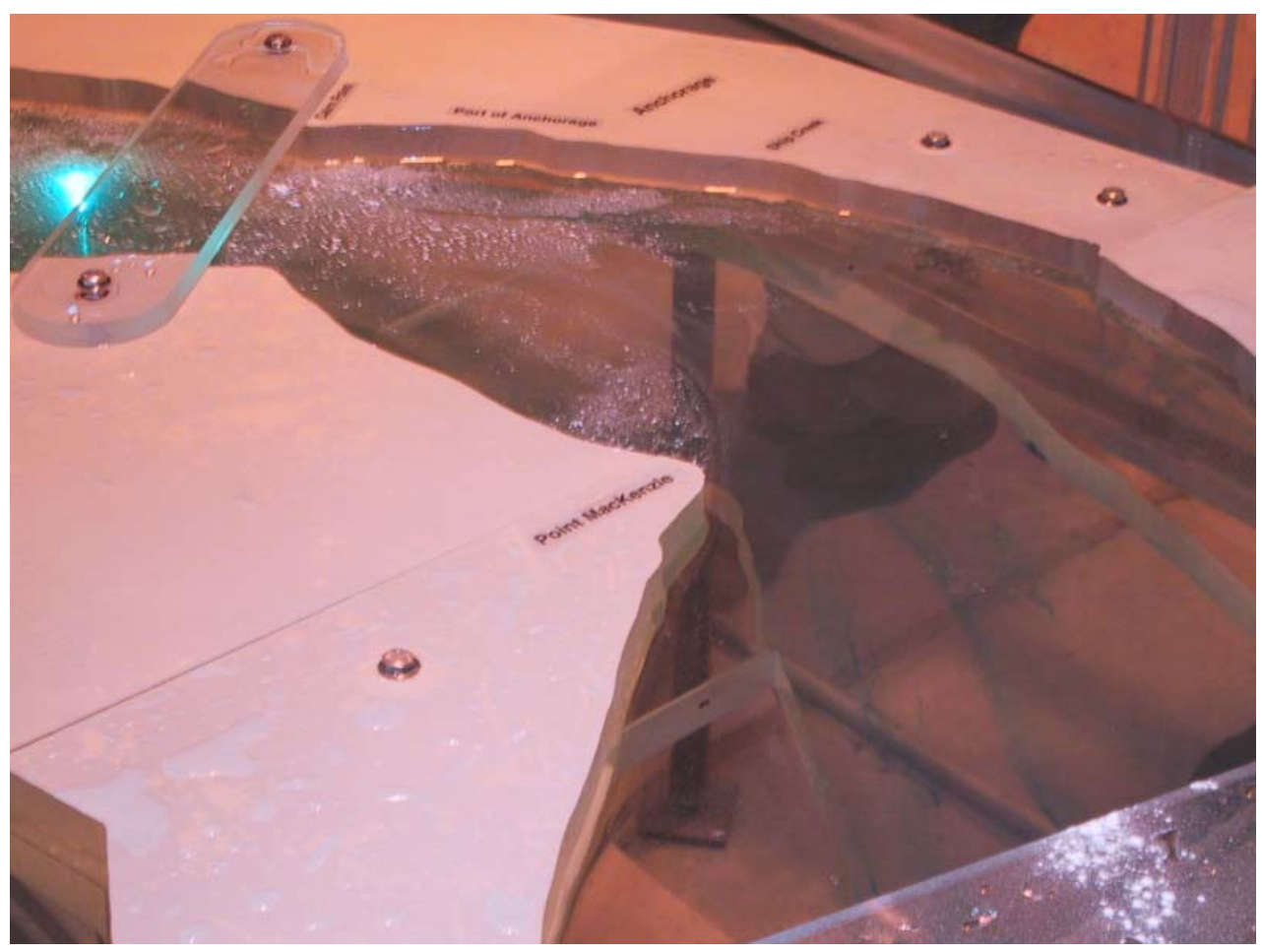

Figure 11. Flood flow separation at Point MacKenzie 


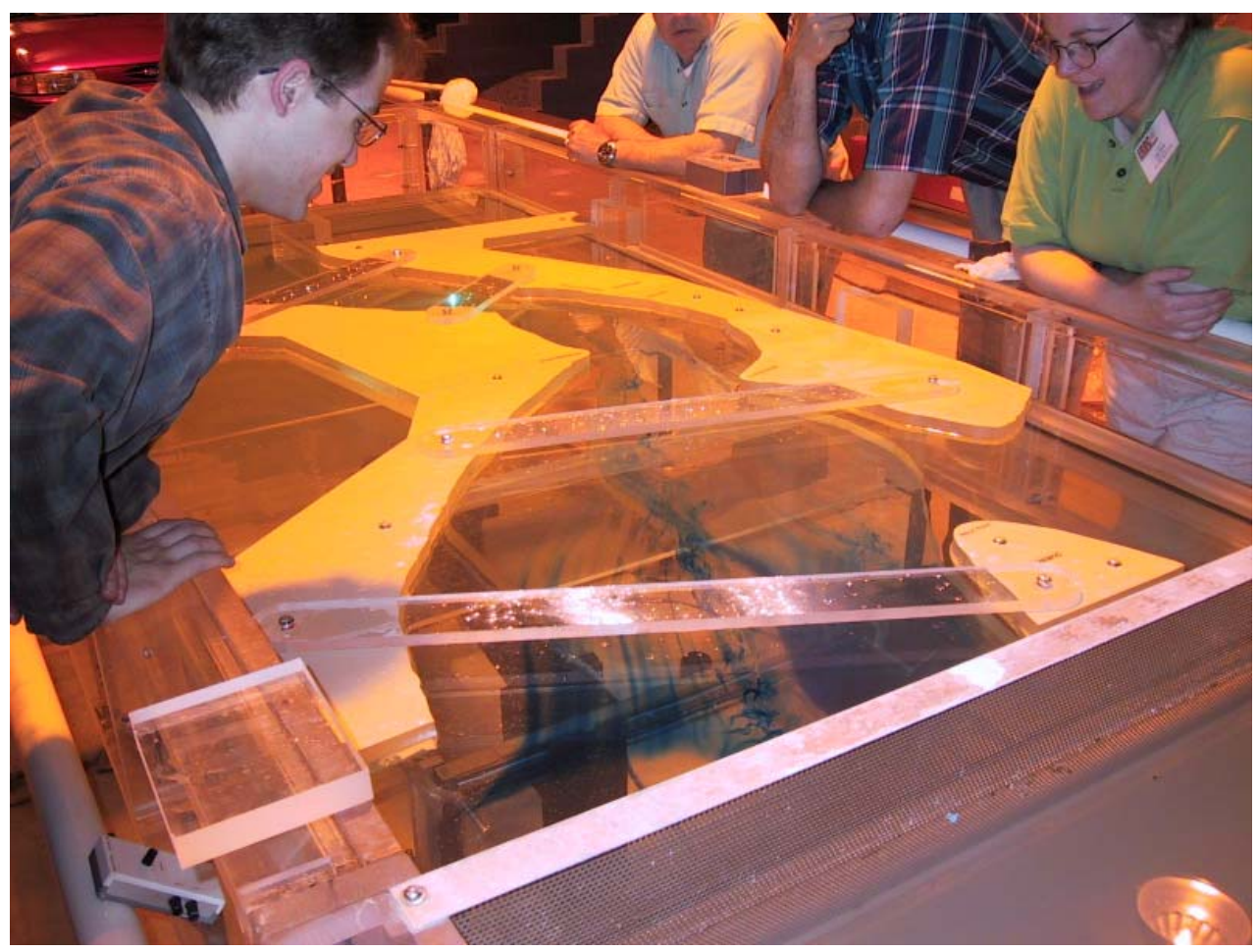

Figure 12. Alaska District engineers observe dye patterns in large-area idealized model

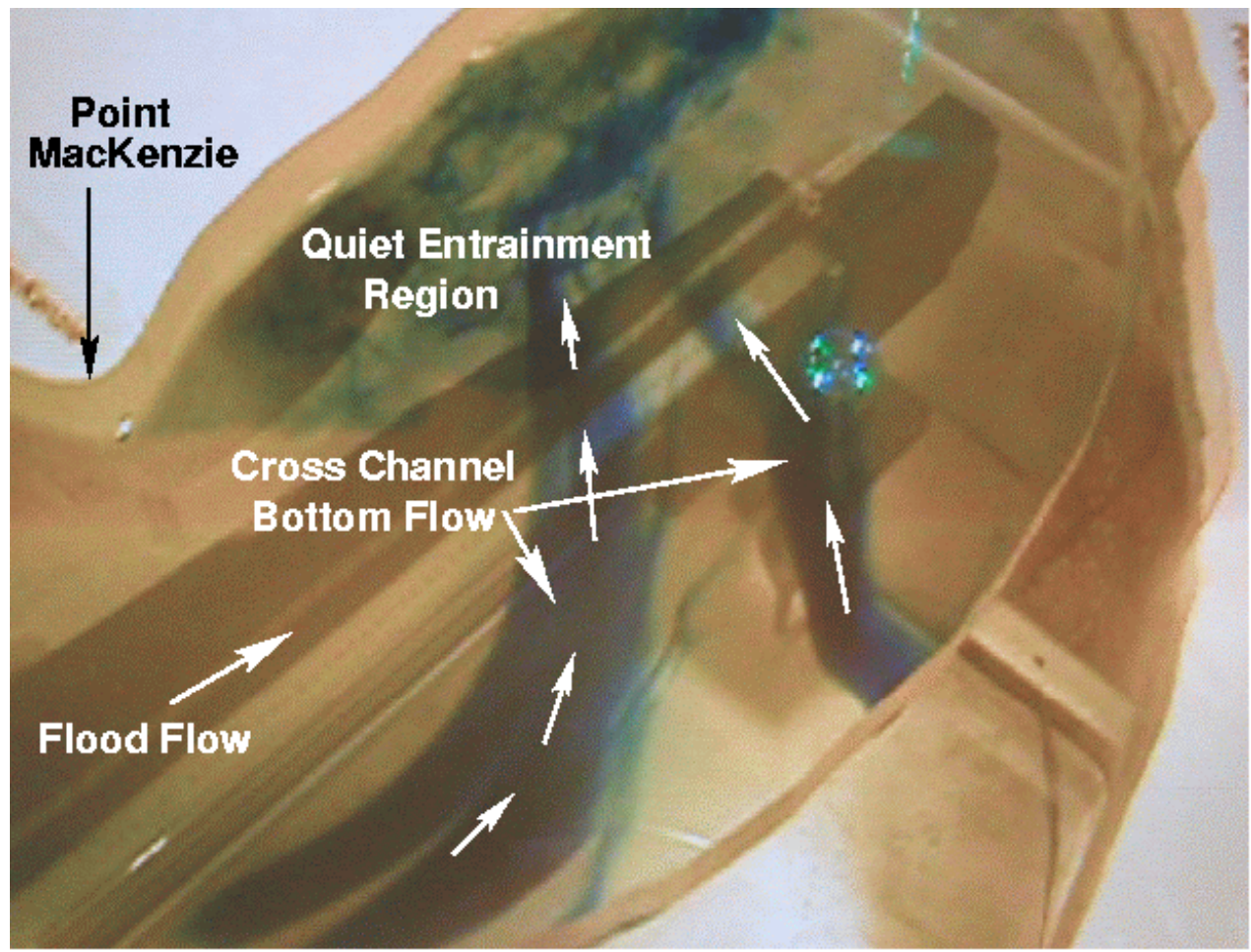

Figure 13. Cross-channel flow at Point MacKenzie during flood tide 
Tracer material was placed in "feeder" piles on the bottom of the model and allowed to move according to prevalent flow patterns. Deposition patterns that formed in the model at the Port of Anchorage and on the Woronzof Shoal were noted by the Alaksa District engineers to be similar to those deposition patterns historically seen at those locations. Deposition of tracer at the Port of Anchorage during flood flow occurred as a crescent-shaped berm starting on one end and extending to the other end. Monthly condition surveys indicated that shoaling at the port occurred in much the same way.

Ebb-tidal simulations with the large-area model reversed on the flow table indicated that Cairn Point plays an important role in sedimentation of the Port of Anchorage. A stationary eddy was formed in the lee of Cairn Point as flow separation occurred at the point. According to the Alaska District engineers,

"The outer edge of the eddy appeared to coincide with the historical development of sediment accumlation in the vicinity of the dock face."

Injected dye captured by the eddy exhibited long residence time in the immediate vicinity of the port. This would gave fine silts in the water column time to settle, thus contributing to sedimentation of the Port of Anchorage.

Testing of the large-area model evolved according to the insights provided by the model with different tide levels being the primary variable. During ebb flow it was shown that modifying the upstream channel configuration could change the geometry of the eddy affecting the Port of Anchorage, but most of the eddy persisted at nearly the same location.

\section{Small-Area Model Description}

The seaward flow boundary of the small-area idealized model was situated just to the west of Point Woronzof with a lateral extent of approximately $3.6 \mathrm{n} . \mathrm{m}$. (Figure 6). The upstream boundary was located about 2.6 n.m. upstream of the Port of Anchorage. This area was scaled to fit roughly into an area with overall dimensions of $0.91 \times 0.91 \mathrm{~m}(3 \mathrm{ft}$ by $3 \mathrm{ft}$ ). Relevant scale ratios for the smallarea model are listed in Table 3 along with approximate model equivalences.

Table 3

Scale Ratios for Small-Area Model

\begin{tabular}{|l|l|l||}
\hline Scale & Scale Value & Model Equivalence \\
\hline \hline Horizontal Scale & $N_{X}=11,307$ & $940 \mathrm{ft} \approx 1 \mathrm{in.}$ \\
\hline Vertical Scale & $N_{Z}=480$ & $40 \mathrm{ft}=1 \mathrm{in.}$ \\
\hline Velocity Scale & $N_{V}=\sqrt{N_{Z}}=21.9$ & $2.2 \mathrm{~m} / \mathrm{sec}=10 \mathrm{~cm} / \mathrm{sec}$ \\
\hline Discharge Scale & $N_{Q}=N_{X} N_{Z}^{3 / 2}=118,907,500$ & $203,000 \mathrm{cu} \mathrm{m} / \mathrm{sec}=1.71$ liters $/ \mathrm{sec}$ \\
\hline
\end{tabular}


Depths in the model were idealized by three horizontal surfaces located at elevations corresponding to depths of el 0 , el -30, and el -60 with vertical transitions between the three depths. The glass bottom of the flow table served as the el -60 contour. Figure 14 shows the small-area model being cut out of 3.81-cm (1.5-in.-) thick Plexiglas, and Figure 15 illustrates the idealized bathymetry in the completed small-area model as viewed from the seaward end.

Contours are shown on Figure 16 where the red lines represent the shoreline, the blue lines are the el 0 , and the green lines are the el -30 depths. As mentioned, the bottom of the flow table represents the el -60 depth. The nearly right-angle bend in the modeled region resulted in the model being built at a size smaller than originally intended to allow a more direct inflow path while still accommodating outflow. The model was designed to be reversed on the table for ebb and flood conditions as illustrated in Figure 17. Operating procedures for the small-area model are the same as described for the large-area model.

\section{Small-Area Model Observations}

The small-area model was also tested extensively during the May 2002 visit by Alaska District engineers. Flow patterns recognized in the large-area model were replicated in the small-area model to see if the more detailed (but still idealized) bathymetry altered any of the flow patterns substantially.

The small area model produced similar flow patterns as the large-area model, particularly the three large flow separation and eddy formation features at Point Woronzof, Point MacKenzie, and Cairn Point. The bottom crosscurrent flowing diagonally between Point Woronzof and Port MacKenzie was also evident.

The small-area model did not replicate the boundaries as far upstream as the large-scale model, and tests showed that changes to these boundaries could have a downstream impact. It was concluded that care must be taken to reproduce accurate upstream boundaries in flow table models so that flow patterns are reasonable facsimiles of the actual region being modeled. This was an obvious constraint in using the small-area model.

The effect of vertical transitions between horizontal layers was investigated by using modeling putty to place a fairing along the vertical transition between the layers. This gave a sloping transition, which allowed vertical mixing to occur between layers. Thus, the main drawback to the idealized bathymetry is prevention of vertical exchange between depths. This becomes a problem only for cases that where 3-D flow structures with non-negligible vertical flow components are known to exist.

\section{Conclusions From Idealized Models}

The two idealized physical models of Cook Inlet proved to be valuable in understanding the complex flow patterns in the vicinity of Anchorage, AK. Even though the models were very small, constructed at a highly distorted geometric scale, and represented fairly complex bathymetry by simple horizontal terraces, 


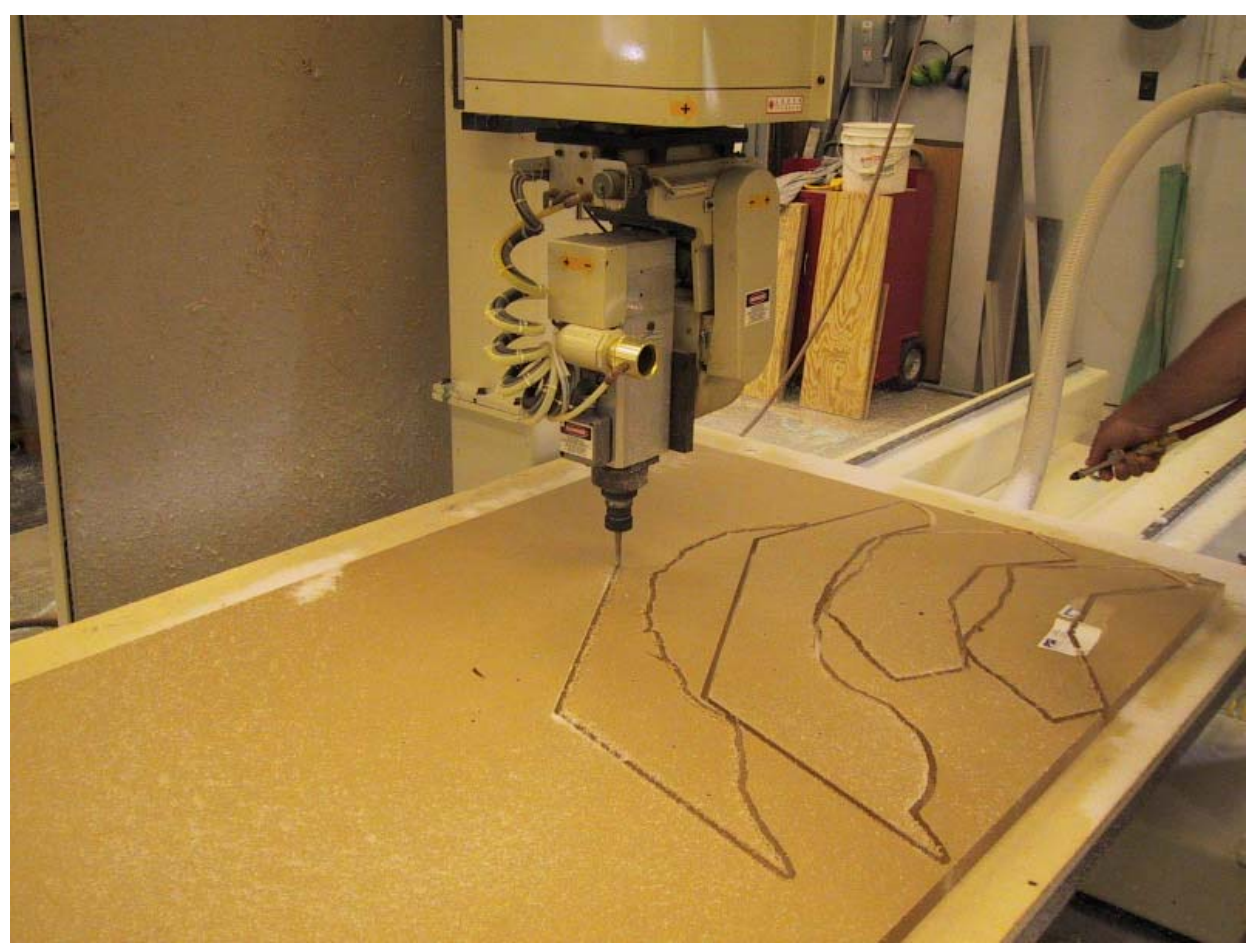

Figure 14. Cutting small-area model pieces with router

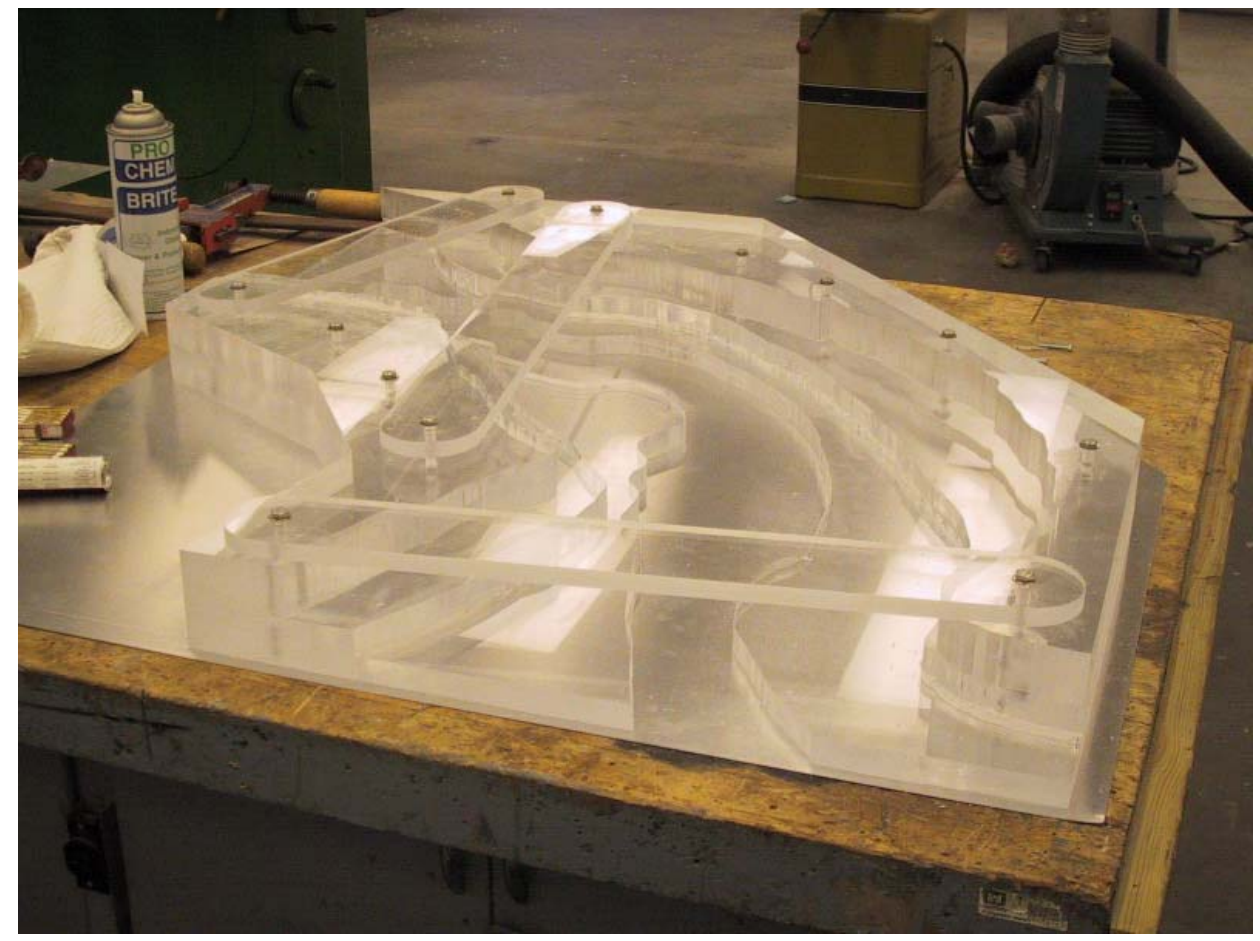

Figure 15. Completed small-area model showing idealized bathymetry 


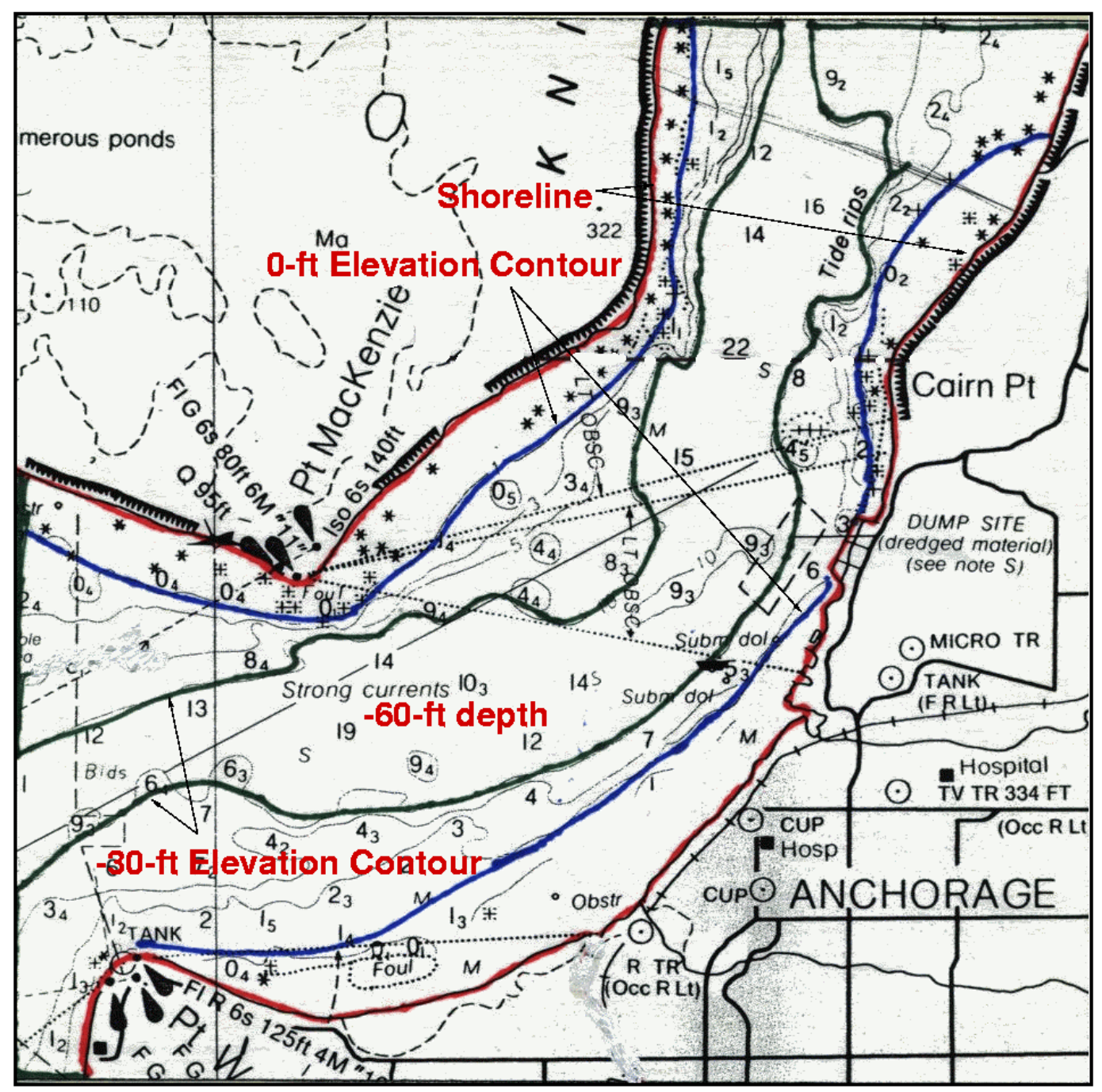

Figure 16. Idealized bathymetry in small-area model 


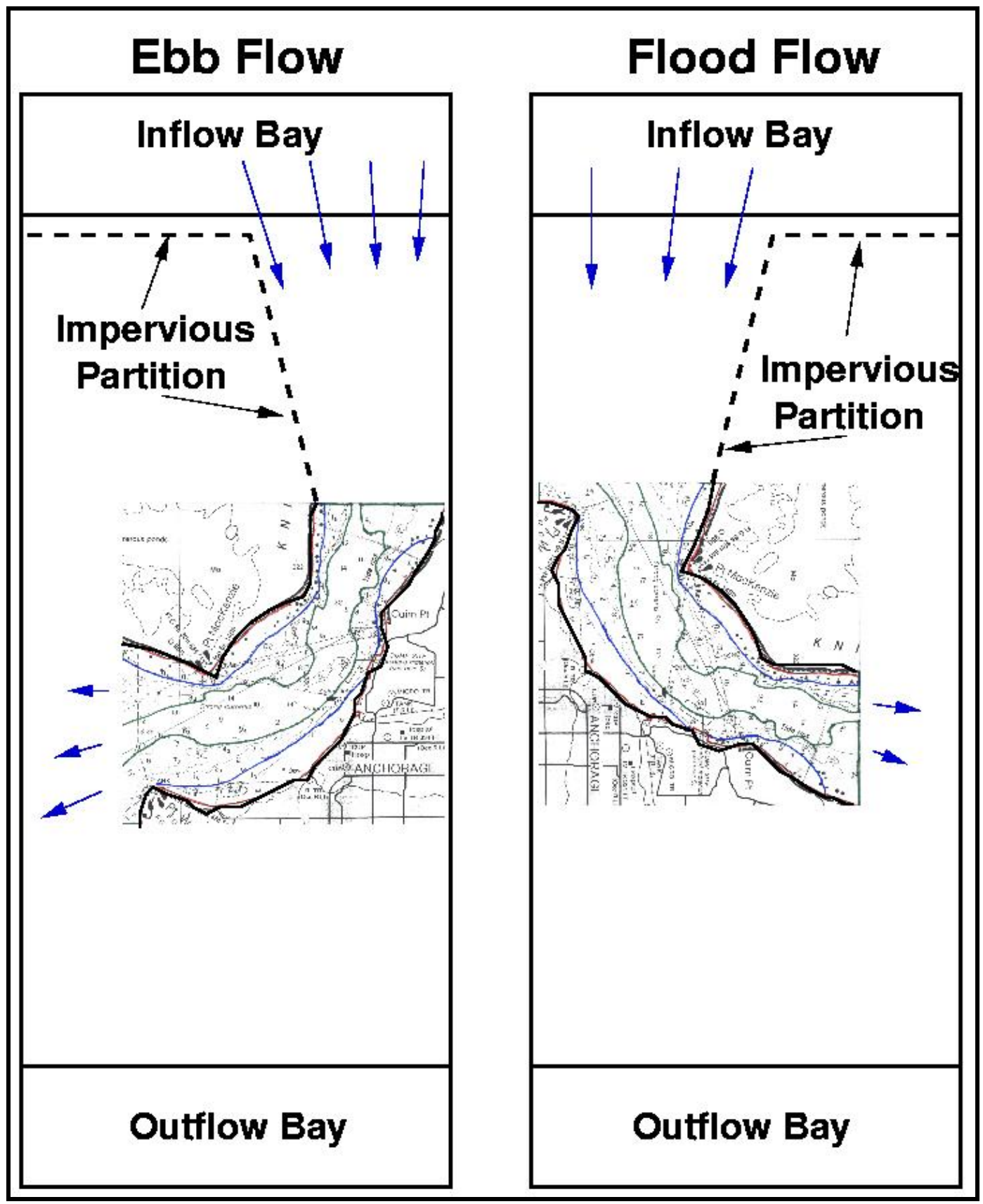

Figure 17. Small-area idealized model positioning on flow table 
they still produced large-scale turbulent flow features known to exist in upper Cook Inlet. Furthermore, the location and areal extent of the gyres and eddies were qualitatively the same as in the prototype. The following conclusions were drawn from the idealized model task based on visual observations, limited velocity measurements (not included in report), and discussions among the test participants:

a. Turbulent flow in Cook Inlet stemming from tidal flow separation at major headlands introduces substantial turbulence into the flow regime with some 3-D effects. Vertical mixing was evident as were crosschannel flows due to water elevation differentials.

$b$. It appeared that the turbulent flow separation at Cairn Point during ebb tide was a significant factor in the sedimentation of the Port of Anchorage, which lies in the lee of the point. Current velocites were reduced to the point that suspended fine sediments had time to settle in the port area.

c. The apparent importance of the 3-D flow components to shoaling at the Port of Anchorage may make application of two-dimensional (2-D), depth-averaged tidal flow numerical models problematic.

d. Upstream boundaries are very important to the flow regime. Consequently, natural processes such as shifting of major shoals could have significant impact that might lead to additional maintenance dredging requirements. Being able to forecast these requirements by monitoring shoal development would be an important achievement.

e. The terraced bathymetry of the idealized models appeared to inhibit water exchange between depth levels. More accurate bathymetry will most certainly produce more reliable flow patterns.

$f$. Geometric distortion of the idealized models will have some impact on the turbulent flow structures, although this impact is difficult to quantify. In general, any vertically-directed turbulent velocities will be greater in the model than in the prototype. However, the steeper slopes in the distorted model will tend to generate less vertical fluid motion. Surface flow characteristics of flow separation and entrainment are in good similitude, but the 3-D structure farther down in the water column may have a scale effect. Flow around bends in the absence of flow separation is reasonably well simulated in a distorted model, and includes more physical reality than depth-averaged numerical models. However, bottom roughness may play a critical role in correct similitude of river bend flows.

g. Idealized flow table models provide useful qualitative insight and semiquantitative results at a modest cost. The key points are to be aware of model limitations and assure that observed flows in the model resemble those observed in nature.

Based on the May 2002 experiments with the idealized models, the Alaska District decided to fund construction of another large-scale flow table model featuring actual bathymetry. Description of the 3-D flow table model and study results are documented in Chapter 7. 


\section{Turbulence Scale Effect in Distorted Models}

\section{Introduction}

Although numerical modeling of free surface flows has shown astonishing growth in the past two decades, one of the few remaining difficulties is simulating turbulent flow processes such as flow around solid bodies, flow separation, and flow entrainment. There are specialized numerical models in developmental stages that can address very localized and specific turbulent processes; but this capability is not generally available in the numerical models typically used to investigate coastal engineering problems.

For many years engineers have used small-scale physical models to study and design coastal engineering projects. The models are useful for understanding and quantifying flow patterns in the vicinity of projects and for predicting changes to flow patterns brought about by project modification. Hydrodynamic forces on solid objects can also be accurately determined using small-scale physical models. Physical modeling of hydrodynamic phenomena is only possible because we have an understanding of how to scale the models. With a properly scaled model, measured and observed responses in the model can be reliably translated to full-scale values.

\section{General Similitude Requirements}

Complete similarity between a physical model and its real-world counterpart (prototype) requires:

a. Geometric similitude. Ratios of all corresponding linear dimensions between prototype and model are the same (i.e., model is a small-scale replica).

b. Kinematic similitude. The ratio between components of all vectorial motions for the prototype and model are the same for all fluid particles at all times.

c. Dynamic similitude. Ratios of all vectorial forces between the prototype and model are the same at all times.

Generally, these three similitude requirements are reasonably met for all freesurface flows in coastal and riverine environments by geometrically undistorted Froude-scaled models. The term "geometrically undistorted" means that the 
model's vertical and horizontal length scales are the same...in other words the model is "geometrically similar."

Undistorted physical models can accurately reproduce the macro-scale features of turbulent processes, and these physical models are the preferred tools for studying turbulence-influenced flows provided the region of interest is small enough and the modeling facility is large enough to accommodate undistorted Froude scaling. When undistorted models are not feasible because of area restrictions, an alternative might be a geometrically distorted physical model, provided the flow conditions meet the necessary criteria.

\section{Geometrically Distorted Models}

In the context of free-surface hydrodynamic physical models, a "geometrically distorted" model is a physical model having a vertical length scale that is different than the horizontal length scale. Geometric distortion, in general, invalidates the necessary scaling criteria; but under very specific conditions the impact of geometric distortion is minimized to the point that model results can be reliably scaled to prototype dimensions. Specifically, situations where the vertical components of flow velocities and accelerations are very small in comparison to the horizontal components can be modeled in distorted models (Hughes 1993). This implies that water pressure is hydrostatic throughout the inviscid flow region. Flow conditions that meet this specific criterion include "long-wave models" (tidal flows) and unidirectional flow models. However, there are certain restrictions that must be observed.

The main advantage of geometrically distorted physical models is the flexibility in choosing the horizontal scale so that large horizontal areas can be modeled in existing model facilities. Without geometric distortion, model water depths would be very small, and model results could be severely affected by surface tension effects and bottom friction. Distorted models allow greater water depths, require less horizontal area, exhibit less frictional losses, and facilitate more accurate vertical measurements (water-surface elevations).

The main drawback to geometrically distorted models is the inability to simulate short waves (wind waves) in addition to long waves. Also, boundary slopes are steeper in distorted models so how this might influence the particular flow situation being modeled must be considered.

In geometrically undistorted models, macro-scale features of turbulence are in similitude with the prototype, so overall characteristics of hydrodynamic processes like breaking waves, hydraulic jumps, and regions of flow separation are faithfully reproduced by the model. Conversely, in geometrically distorted models, turbulent processes are not in strict theoretical similitude.

For most distorted physical models, this is generally not a problem either because the modeled flow situation does not have significant large-scale turbulence, or because the turbulent processes that do occur have little impact on the flow features being simulated. However, if flow patterns in the main region 
of interest are strongly influenced by turbulence, a scale effect ${ }^{1}$ will be present in a distorted model.

If distorted physical models of turbulent flow regions are to be a viable alternative, the turbulence scale effect must be assessed, and a determination must be made on how the scale effect will modify model results that are scaled up to full-size. This understanding could expand the utility of physical hydrodynamic models for simulating real-world coastal engineering problems.

\section{Turbulence Similitude in Geometrically Distorted Models}

Strict similitude criteria for hydrodynamic modeling are found by casting the Navier-Stokes equations into nondimensional form and requiring that all dimensionless coefficients retain the same value in the model as in the prototype. Following the derivation provided by Hughes (1993), the four govening equations for incompressible, free surface flow are given by the continuity equation and the Navier-Stokes equations, i.e.,

continuity

$$
\frac{\partial u}{\partial x}+\frac{\partial v}{\partial y}+\frac{\partial w}{\partial z}=0
$$

$\mathrm{x}$-direction momentum

$$
\begin{aligned}
& \underbrace{\frac{\partial u}{\partial t}+u \frac{\partial u}{\partial x}+v \frac{\partial u}{\partial y}+w \frac{\partial u}{\partial z}}_{\text {acceleration terms }}=\underbrace{-\frac{1}{\rho} \frac{\partial p}{\partial x}}_{\text {pressure term }}+\underbrace{v\left(\frac{\partial^{2} u}{\partial x^{2}}+\frac{\partial^{2} u}{\partial y^{2}}+\frac{\partial^{2} u}{\partial z^{2}}\right)}_{\text {viscous shear terms }} \\
& -\underbrace{\left[\frac{\partial}{\partial x}\left(\overline{u^{\prime 2}}\right)+\frac{\partial}{\partial y}\left(\overline{u^{\prime} v^{\prime}}\right)+\frac{\partial}{\partial z}\left(\overline{u^{\prime} w^{\prime}}\right)\right]}_{\text {turbulence terms }}
\end{aligned}
$$

\footnotetext{
${ }^{1}$ Scale effects are differences between the prototype and model response that arise from the inability to simulate all relevant forces in the model at the proper scale dictated by the scaling criteria.
} 
y-direction momentum

$$
\begin{aligned}
& \underbrace{\frac{\partial v}{\partial t}+u \frac{\partial v}{\partial x}+v \frac{\partial v}{\partial y}+w \frac{\partial v}{\partial z}}_{\text {acceleration terms }}=\underbrace{-\frac{1}{\rho} \frac{\partial p}{\partial y}}_{\text {pressure term }}+\underbrace{v\left(\frac{\partial^{2} v}{\partial x^{2}}+\frac{\partial^{2} v}{\partial y^{2}}+\frac{\partial^{2} v}{\partial z^{2}}\right)}_{\text {viscous shear terms }} \\
& -\underbrace{\left[\frac{\partial}{\partial x}\left(\overline{u^{\prime} v^{\prime}}\right)+\frac{\partial}{\partial y}\left(\overline{v^{\prime 2}}\right)+\frac{\partial}{\partial z}\left(\overline{v^{\prime} w^{\prime}}\right)\right]}_{\text {turbulence terms }}
\end{aligned}
$$

z-direction momentum

$$
\begin{aligned}
& \underbrace{\frac{\partial w}{\partial t}+u \frac{\partial w}{\partial x}+v \frac{\partial w}{\partial y}+w \frac{\partial w}{\partial z}}_{\text {acceleration terms }}=\underbrace{-\frac{1}{\rho} \frac{\partial p}{\partial z}}_{\text {pressure term }} \underbrace{-g}_{\text {gravity }}+\underbrace{v\left(\frac{\partial^{2} w}{\partial x^{2}}+\frac{\partial^{2} w}{\partial y^{2}}+\frac{\partial^{2} w}{\partial z^{2}}\right)}_{\text {viscous shear terms }} \\
& -\underbrace{\left[\frac{\partial}{\partial x}\left(\overline{u^{\prime} w^{\prime}}\right)+\frac{\partial}{\partial y}\left(\overline{v^{\prime} w^{\prime}}\right)+\frac{\partial}{\partial z}\left(\overline{w^{\prime 2}}\right)\right]}_{\text {turbulence terms }}
\end{aligned}
$$

where

$$
\begin{aligned}
t= & \text { time } \\
x, y= & \text { horizontal coordinates } \\
z= & \text { vertical coordinate } \\
u, v= & \text { nonturbulent horizontal components of velocity in the } \mathrm{x} \text { and } \mathrm{y} \\
& \text { directions, respectively } \\
u^{\prime}, v^{\prime}= & \text { turbulent fluctuating components of velocity in the } \mathrm{x} \text { and } \mathrm{y} \text { directions }, \\
& \text { respectively } \\
w= & \text { nonturbulent vertical component of velocity in the } \mathrm{z} \text { direction } \\
w^{\prime}= & \text { turbulent fluctuating component of velocity in the } \mathrm{z} \text { direction } \\
g= & \text { gravitational acceleration } \\
p= & \text { fluid pressure } \\
\rho= & \text { fluid density } \\
v= & \text { fluid kinematic viscosity }
\end{aligned}
$$

Appropriate scaling criteria are derived from the equations of motion by expressing the equations in nondimensional form using the following definitions and substituting for the independent and dependent variables: 


$$
\begin{array}{rlrl}
\hat{u}=\frac{u}{V} ; & \hat{v}=\frac{v}{V} ; & \hat{w}=\frac{w}{W} \\
\hat{x}=\frac{x}{X} ; & \hat{y}=\frac{y}{X} ; & \hat{z}=\frac{z}{Z} \\
\hat{t}=\frac{t}{T} ; & \hat{p}=\frac{p}{P}
\end{array}
$$

where

$$
\begin{aligned}
V & =\text { characteristic nonturbulent horizontal velocity } \\
W & =\text { characteristic nonturbulent vertical velocity } \\
X & =\text { characteristic horizontal length } \\
Z & =\text { characteristic vertical length } \\
T & =\text { characteristic time } \\
P & =\text { characteristic pressure }
\end{aligned}
$$

Different characteristic lengths and velocities were chosen for the horizontal and vertical directions to accommodate geometrically distorted hydrodynamic models. For convenience, the same characteristic velocities were used to nondimensionalize the turbulent Reynolds stresses.

Making these substitutions into Equations 1 through 5, multiplying the continuity equation by $X / V$, the horizontal momentum equations by $X / V^{2}$, and the vertical momentum equation by $Z / W^{2}$ yields the nondimensional equations of motion.

continuity (nondimensional)

$$
\frac{\partial \hat{u}}{\partial \hat{x}}+\frac{\partial \hat{v}}{\partial \hat{y}}+\left(\frac{X W}{Z V}\right) \frac{\partial \hat{w}}{\partial \hat{z}}=0
$$

$\mathrm{x}$-direction momentum (nondimensional)

$$
\begin{aligned}
\left(\frac{X}{V T}\right) \frac{\partial \hat{u}}{\partial \hat{t}}+ & \hat{u} \frac{\partial \hat{u}}{\partial \hat{x}}+\hat{v} \frac{\partial \hat{u}}{\partial \hat{y}}+\left(\frac{X W}{Z V}\right) \hat{w} \frac{\partial \hat{u}}{\partial \hat{z}}=-\left(\frac{P}{\rho V^{2}}\right) \frac{\partial \hat{p}}{\partial \hat{x}} \\
+ & {\left[\left(\frac{v}{X V}\right) \frac{\partial^{2} \hat{u}}{\partial \hat{x}^{2}}+\left(\frac{v}{X V}\right) \frac{\partial^{2} \hat{u}}{\partial \hat{y}^{2}}+\left(\frac{v X}{Z^{2} V}\right) \frac{\partial^{2} \hat{u}}{\partial \hat{z}^{2}}\right] } \\
& -\underbrace{\left[\frac{\partial}{\partial \hat{x}}\left(\overline{\hat{u}^{\prime 2}}\right)+\frac{\partial}{\partial \hat{y}}\left(\overline{\hat{u}^{\prime} \hat{v}^{\prime}}\right)+\left(\frac{X W}{Z V}\right) \frac{\partial}{\partial \hat{z}}\left(\overline{\hat{u}^{\prime} \hat{w}^{\prime}}\right)\right]}_{\text {turbulence terms }}
\end{aligned}
$$


y-direction momentum (nondimensional)

$$
\begin{aligned}
\left(\frac{X}{V T}\right) \frac{\partial \hat{v}}{\partial \hat{t}}+ & \hat{u} \frac{\partial \hat{v}}{\partial \hat{x}}+\hat{v} \frac{\partial \hat{v}}{\partial \hat{y}}+\left(\frac{X W}{Z V}\right) \hat{w} \frac{\partial \hat{v}}{\partial \hat{z}}=-\left(\frac{P}{\rho V^{2}}\right) \frac{\partial \hat{p}}{\partial \hat{y}} \\
+ & {\left[\left(\frac{v}{X V}\right) \frac{\partial^{2} \hat{v}}{\partial \hat{x}^{2}}+\left(\frac{v}{X V}\right) \frac{\partial^{2} \hat{v}}{\partial \hat{y}^{2}}+\left(\frac{v X}{Z^{2} V}\right) \frac{\partial^{2} \hat{v}}{\partial \hat{z}^{2}}\right] } \\
& -\underbrace{\left[\frac{\partial}{\partial \hat{x}}\left(\overline{\hat{u}^{\prime} \hat{v}^{\prime}}\right)+\frac{\partial}{\partial \hat{y}}\left(\overline{\hat{v}^{\prime 2}}\right)+\left(\frac{X W}{Z V}\right) \frac{\partial}{\partial \hat{z}}\left(\overline{\hat{v}^{\prime} \hat{w}^{\prime}}\right)\right]}_{\text {turbulence terms }}
\end{aligned}
$$

Z-direction momentum (nondimensional)

$$
\begin{aligned}
\left(\frac{Z}{W T}\right) \frac{\partial \hat{w}}{\partial \hat{t}} & +\left(\frac{Z V}{X W}\right)\left(\hat{u} \frac{\partial \hat{w}}{\partial \hat{x}}+\hat{v} \frac{\partial \hat{w}}{\partial \hat{y}}\right)+\hat{w} \frac{\partial \hat{w}}{\partial \hat{z}}=-\left(\frac{P}{\rho W^{2}}\right) \frac{\partial \hat{p}}{\partial \hat{z}} \\
& -\left(\frac{g Z}{W^{2}}\right)+\left[\left(\frac{v Z}{X^{2} V}\right) \frac{\partial^{2} \hat{w}}{\partial \hat{x}^{2}}+\left(\frac{v Z}{X^{2} W}\right) \frac{\partial^{2} \hat{w}}{\partial \hat{y}^{2}}+\left(\frac{v}{Z W}\right) \frac{\partial^{2} \hat{w}}{\partial \hat{z}^{2}}\right] \\
& -\underbrace{\left[\left(\frac{Z V}{X W}\right)\left(\frac{\partial}{\partial \hat{x}}\left(\overline{\hat{u}^{\prime} \hat{w}^{\prime}}\right)+\frac{\partial}{\partial \hat{y}}\left(\overline{\hat{v}^{\prime} \hat{w}^{\prime}}\right)\right)+\frac{\partial}{\partial \hat{z}}\left(\overline{\hat{w}^{\prime 2}}\right)\right]}
\end{aligned}
$$

If two systems are governed by the previous nondimensional equations, then the solution in terms of the nondimensional parameters will be the same for each system provided all dimensionless coefficients remain unchanged. This means complete similitude would be achieved for any free surface hydrodynamic phenomena governed by the formulation of the Navier-Stokes equations if the value of each dimensionless coefficient in Equations 6 through 9 remains constant between prototype and model. Note that all nondimensional terms without coefficients will be in similitude.

Focusing attention on those groupings labeled "turbulence terms," the only dimensionless coefficients are $(X W / Z V)$ and its inverse. Therefore, the requirement for similitude of the differential turbulence Reynolds stress terms having this coefficient is simply

$$
\left(\frac{X W}{Z V}\right)_{\text {prototype }}=\left(\frac{X W}{Z V}\right)_{\text {model }}
$$

or rearranged...

$$
\left(\frac{X_{p}}{X_{m}}\right)\left(\frac{W_{p}}{W_{m}}\right)=\left(\frac{Z_{p}}{Z_{m}}\right)\left(\frac{V_{p}}{V_{m}}\right)
$$


where the subscripts $p$ and $m$ represent prototype and model, respectively. The requirement given by Equation 10 can be expressed in terms of scale ratios as:

$$
\frac{N_{X}}{N_{Z}}=\frac{N_{V}}{N_{W}}
$$

where, by definition, a scale ratio is the ratio of a parameter in the prototype to the value of the same parameter in the model. ${ }^{1}$

Because the scaling ratio for horizontal and vertical velocity should be the same, the scaling requirement of Equation 4-12 can only be fulfilled when $N_{X}=$ $N_{Z}$, which is the requirement for a geometrically undistorted physical model. Therefore, turbulent Reynolds stress terms containing this dimensionless coefficient will not be in similitude in a geometrically distorted model, and this introduces a scale effect. From Equations 4-6 through 4-9 the following can be concluded:

turbulence terms in similitude

$$
\frac{\partial}{\partial x}\left(\overline{u^{\prime 2}}\right) ; \frac{\partial}{\partial y}\left(\overline{u^{\prime} v^{\prime}}\right) ; \frac{\partial}{\partial x}\left(\overline{u^{\prime} v^{\prime}}\right) ; \frac{\partial}{\partial y}\left(\overline{v^{\prime 2}}\right) ; \frac{\partial}{\partial z}\left(\overline{w^{\prime 2}}\right)
$$

turbulence terms not in similitude

$$
\frac{\partial}{\partial x}\left(\overline{u^{\prime} w^{\prime}}\right) ; \frac{\partial}{\partial y}\left(\overline{v^{\prime} w^{\prime}}\right) ; \frac{\partial}{\partial z}\left(\overline{u^{\prime} w^{\prime}}\right) ; \frac{\partial}{\partial z}\left(\overline{v^{\prime} w^{\prime}}\right)
$$

The four turbulence terms involving squares or cross products of the horizontal turbulent velocity fluctuations and the term containing the square of the vertical turbulent velocity fluctuation are in similitude in distorted models, whereas the four Reynolds stress terms containing the cross-product of horizontal and vertical velocities do not fulfill the requirement and represent the potential scale effects.

The two nonsimilar turbulent Reynolds stress terms contained in the horizontal momentum equations are larger in the model than they should be by a factor equal to the geometric distortion, i.e.,

\footnotetext{
${ }^{1}$ For example, the scale ratio of the characteristic horizontal length $X$ is:

$$
N_{X}=\frac{X_{p}}{X_{m}}=\frac{\text { Value of } \mathrm{X} \text { in prototype }}{\text { Value of } \mathrm{X} \text { in model }}
$$
}




$$
\Omega\left[\frac{\partial}{\partial \hat{z}}\left(\overline{\hat{u}^{\prime} \hat{w}^{\prime}}\right) ; \frac{\partial}{\partial \hat{z}}\left(\overline{\hat{v}^{\prime} \hat{w}^{\prime}}\right)\right]_{\text {proto }}=\left[\frac{\partial}{\partial \hat{z}}\left(\overline{\hat{u}^{\prime} \hat{w}^{\prime}}\right) ; \frac{\partial}{\partial \hat{z}}\left(\overline{\hat{v}^{\prime} \hat{w}^{\prime}}\right)\right]_{\text {model }}
$$

where $\Omega=N_{X} / N_{Z}$. This implies that the vertical variation of the turbulent velocity products $u^{\prime} w^{\prime}$ and $v^{\prime} w^{\prime}$ is greater in the distorted model than in the prototype.

Conversely, the two nonsimilar turbulent Reynolds stress terms contained in the vertical momentum equation are smaller in the model than they should be by a factor equal to the inverse of the geometric distortion, i.e.,

$$
\frac{1}{\Omega}\left[\frac{\partial}{\partial \hat{x}}\left(\overline{\hat{u}^{\prime} \hat{w}^{\prime}}\right) ; \frac{\partial}{\partial \hat{y}}\left(\overline{\hat{v}^{\prime} \hat{w}^{\prime}}\right)\right]_{\text {proto }}=\left[\frac{\partial}{\partial \hat{x}}\left(\overline{\hat{u}^{\prime} \hat{w}^{\prime}}\right) ; \frac{\partial}{\partial \hat{y}}\left(\overline{\hat{v}^{\prime} \hat{w}^{\prime}}\right)\right]_{\text {model }}
$$

In this case the horizontal variation of the turbulent velocity products $\bar{w}^{\prime}$ and $v^{\prime} w^{\prime}$ is less in the distorted model than in the prototype.

\section{Anticipated Scale Effects}

Several generalizations can be made regarding anticipated turbulent scale effects in geometrically distorted models by assuming the turbulence is homogeneous in the horizontal and vertical directions. As mentioned, turbulence generated by flow interaction with solid boundaries will be in similitude in a geometrically undistorted physical model.

As an example, consider a fluid jet exiting a circular orifice into an ambient fluid. The turbulent jet spreads out uniformly in both horizontal and vertical directions with distance from the orifice. An undistorted model of this jet would also have a circular orifice and would also spread out uniformly in the downstream direction. It should be expected that time-averaged velocity measurements taken at any location in the model turbulent jet and scaled using the Froude velocity scale would be the same as averaged velocities measured at the corresponding location in the prototype.

The same circular orifice would be represented in a distorted model as an oval with its major axis aligned vertically. As the jet exits the orifice, it will form an oval-shaped turbulent jet. The jet will expand, and anticipate its expansion to be the same in the vertical plane as in the horizontal plane as mass is entrained into the jet. This means the lateral vertical and horizontal entrainment velocity is the same in the model. However, the distorted model implies that vertical and horizontal velocity scales are different. Therefore, when the time-averaged velocities are scaled to prototype and compared to averages at the corresponding location, expect to see a difference due to dissimilar lateral entrainment velocity. The magnitude of the error would not be too significant because the principle flow direction is horizontal. The scaled-up cross-jet velocity profiles would be similar to the prototype in magnitude, but we should expect some error in the profile shape. 
The impact of the turbulence scale effect depends on the relative magnitudes of the turbulence in the horizontal and vertical planes. The smallest turbulence scale effect will occur in situations where the turbulence is manifested primarily in either the horizontal or vertical plane. As an example, numerous observations of plane turbulent jets exiting a channel of width $2 b_{o}$ between two vertical edges have shown that the downstream mean horizontal velocity $u_{m}$ along the jet center line is well represented by a self-similar empirical formula such as:

$$
u_{m}=3.5 U_{o} \sqrt{\frac{b_{o}}{x}}
$$

where $U_{o}$ is the exit velocity and $x$ is the distance downstream. This relationship is valid for both geometrically distorted and undistorted flow models at any depth in the jet, which indicates that the turbulence is primarily in the horizontal plane with only weak fluctuations in the vertical plane. In other words, for the case of a plane jet exiting a channel with vertical sidewalls, the turbulence terms that are not in similitude in distorted models have only minor influence because the vertical velocity fluctuations are small compared to the horizontal fluctuations.

A similar jet exiting a channel with sloping edges will have vertical velocity fluctuations on the same order as (or even greater than) the horizontal fluctuations. Do not expect self-similar behavior in the downstream jet at different water depths because the induced vertical velocities impact the entrainment rate and transport mass vertically. In a distorted model the sloping edges of the channel creating the jet will be steeper. This will decrease the vertical velocity fluctuations, making the jet structure appear more like that of a plane jet with vertical channel walls.

The magnitude of the turbulence scale effect in distorted models will vary with each situation and the amount of model distortion. For some studies the effect may be negligible so long as the major flow features are reasonably reproduced. For example, if there is a region of flow separation in the lee of a headland, expect the distorted model to reproduce the geometry of the flow separation downstream of the headland (particularly on the surface), and the maximum velocity magnitudes and directions caused by flow accelerations along the line of separation should scale reasonably correct. Within the area of reduced flow adjacent to the line of flow separation, velocity vectors may not be in similitude with the prototype, but this might not be as important so long as it recognized that this might be an area where sedimentation could take place. Any observed vertical velocities are likely to be more pronounced in the distorted model, but on the other hand, the steeper slopes in the distorted model will produce smaller vertical velocities so this could help balance the disparity.

Flow around a vertical cylinder represents an interesting case because of the well-known horseshoe vortex responsible for scour. This vortex has strong vertical velocity and acceleration components that will not be well represented in a distorted model. In some physical models of inlets and harbors, vertical piles obstruct the flow, and it is important to establish how well this flow reduction is simulated in distorted models. Vortices are shed in the wake of the pile, but 
these are largely manifested in the horizontal plane over most of the water column. It is expected that the shed vortices will be in approximate similitude in a geometrically distorted physical model. Experiments conducted to assess potential turbulence scaling effects in distorted models are discussed in the following chapter.

\section{Flow at Bend}

Finally, in addition to scale effects related to generation of turbulence, any mechanism that creates significant vertical velocities or accelerations will be problematic in a geometrically distorted model. For example, at river bends centrifugal forces pile water up on the outside of the bend, which creates a lateral slope in the water surface. The force imbalance results in a cross-channel return flow toward the inside of the curve along the bottom. The resulting secondary flow resembles a helix as it moves downstream. In a distorted model the curve geometry will be tighter and the side slopes will be steeper. The same phenomenon occurs where flow accelerates as it passes a headland or jetty causing a lowering of the local water surface.

Similitude requirements are formally determined as before, only this time the following governing equations are presented in cylindrical coordinates as often adopted for numerical modeling of flow around river bends. Turbulence terms and viscous shear stress terms have been omitted from the momentum equations to focus on the distorted model scale effects associated with flow acceleration at a river bend.

continuity

$$
\frac{\partial v_{r}}{\partial r}+\frac{v_{r}}{r}+\frac{1}{r} \frac{\partial v_{\theta}}{\partial \theta}+\frac{\partial v_{z}}{\partial z}=0
$$

r-direction momentum

$$
\underbrace{\frac{\partial v_{r}}{\partial t}+v_{r} \frac{\partial v_{r}}{\partial r}+\frac{v_{\theta}}{r} \frac{\partial v_{r}}{\partial \theta}-\frac{v_{\theta}^{2}}{r}+v_{z} \frac{\partial v_{r}}{\partial z}}_{\text {acceleration terms }}=\underbrace{-\frac{1}{\rho} \frac{\partial p}{\partial r}}_{\text {pressure term }}
$$

$\theta$-direction momentum

$$
\underbrace{\frac{\partial v_{\theta}}{\partial t}+v_{r} \frac{\partial v_{\theta}}{\partial r}+\frac{v_{\theta}}{r} \frac{\partial v_{\theta}}{\partial \theta}+\frac{v_{r} v_{\theta}}{r}+v_{z} \frac{\partial v_{\theta}}{\partial z}}_{\text {acceleration terms }}=\underbrace{-\frac{1}{\rho r} \frac{\partial p}{\partial \theta}}_{\text {pressure term }}
$$


z-direction momentum

$$
\underbrace{\frac{\partial v_{z}}{\partial t}+v_{r} \frac{\partial v_{z}}{\partial r}+\frac{v_{\theta}}{r} \frac{\partial v_{z}}{\partial \theta}+v_{z} \frac{\partial v_{z}}{\partial z}}_{\text {acceleration terms }}=\underbrace{-\frac{1}{\rho} \frac{\partial p}{\partial z}}_{\text {pressure term }}+\underbrace{g}_{\text {gravity }}
$$

where

$t=$ time

$r=$ horizontal cross-channel (radial) coordinate

$\theta=$ horizontal along-channel (angular) coordinate

$z=$ vertical coordinate

$v_{r}=$ nonturbulent horizontal cross-channel component of velocity in the $\mathrm{r}$ direction

$v_{6}=$ nonturbulent horizontal along-channel component of velocity in the $\theta$ direction

$v_{z}=$ nonturbulent vertical component of velocity in the $\mathrm{z}$ direction

$g=$ gravitational acceleration

$p=$ fluid pressure

$\rho=$ fluid density

Appropriate scaling criteria are derived from the equations of motion by expressing the equations in nondimensional form using the following definitions and substituting for the independent and dependent variables:

$$
\begin{array}{rlrl}
\hat{v}_{r}=\frac{v_{r}}{V} ; & \hat{v}_{\theta}=\frac{v_{\theta}}{V} ; & \hat{v}_{z}=\frac{v_{z}}{V} ; & \hat{t}=\frac{t}{T} \\
\hat{r}=\frac{r}{X} ; & \hat{z}=\frac{z}{Z} & \hat{p}=\frac{p}{P}
\end{array}
$$

where

$$
\begin{aligned}
& V=\text { characteristic nonturbulent horizontal velocity } \\
& X=\text { characteristic horizontal length } \\
& Z=\text { characteristic vertical length } \\
& T=\text { characteristic time } \\
& P=\text { characteristic pressure }
\end{aligned}
$$


Different characteristic lengths were chosen for the horizontal and vertical directions to accommodate geometrically distorted hydrodynamic models. For convenience the same characteristic velocity was used to nondimensionalize the radial, tangential, and vertical velocity components.

Making these substitutions into Equations 18 through 21, multiplying the continuity equation by $X / V$, the horizontal momemtum equations by $X / V^{2}$, and the vertical momentum equation by $Z / V^{2}$ yields the nondimensional equations of motion in cylindrical coordinates.

continuity (nondimensional)

$$
\frac{\partial \hat{v}_{r}}{\partial \hat{r}}+\frac{\hat{v}_{r}}{\hat{r}}+\frac{1}{\hat{r}} \frac{\partial \hat{v}_{\theta}}{\partial \theta}+\left(\frac{X}{Z}\right) \frac{\partial \hat{v}_{z}}{\partial \hat{z}}=0
$$

$\mathrm{x}$-direction momentum (nondimensional)

$$
\left(\frac{X}{V T}\right) \frac{\partial \hat{v}_{r}}{\partial \hat{t}}+\underbrace{\hat{v}_{r} \frac{\partial \hat{v}_{r}}{\partial \hat{r}}+\frac{\hat{v}_{\theta}}{\hat{r}} \frac{\partial \hat{v}_{r}}{\partial \theta}-\frac{\hat{v}_{\theta}^{2}}{\hat{r}}+\left(\frac{X}{Z}\right) \hat{v}_{z} \frac{\partial \hat{v}_{r}}{\partial \hat{z}}}_{\text {convective accelerations }}=-\left(\frac{P}{\rho V^{2}}\right) \frac{\partial \hat{p}}{\partial \hat{r}}
$$

$\theta$-direction momentum (nondimensional)

$$
\left(\frac{X}{V T}\right) \frac{\partial \hat{v}_{\theta}}{\partial \hat{t}}+\underbrace{\hat{v}_{r} \frac{\partial \hat{v}_{\theta}}{\partial \hat{r}}+\frac{\hat{v}_{\theta}}{\hat{r}} \frac{\partial \hat{v}_{\theta}}{\partial \theta}+\frac{\hat{v}_{r} \hat{v}_{\theta}}{\hat{r}}+\left(\frac{X}{Z}\right) \hat{v}_{z} \frac{\partial \hat{v}_{\theta}}{\partial \hat{z}}}_{\text {convective accelerations }}=-\left(\frac{P}{\rho V^{2}}\right) \frac{1}{\hat{r}} \frac{\partial \hat{p}}{\partial \theta}
$$

Z-direction momentum (nondimensional)

$$
\left(\frac{Z}{V T}\right) \frac{\partial \hat{v}_{z}}{\partial \hat{t}}+\left(\frac{Z}{X}\right) \hat{v}_{r} \frac{\partial \hat{v}_{z}}{\partial \hat{r}}+\left(\frac{Z}{X}\right) \frac{\hat{v}_{\theta}}{\hat{r}} \frac{\partial \hat{v}_{z}}{\partial \theta}+\hat{v}_{z} \frac{\partial \hat{v}_{z}}{\partial \hat{z}}=-\left(\frac{P}{\rho V^{2}}\right) \frac{\partial \hat{p}}{\partial \hat{z}}+\left(\frac{g Z}{V^{2}}\right)
$$

Just as before, if two systems are governed by the nondimensional equations, then the solution in terms of the nondimensional parameters will be the same for each system provided all dimensionless coefficients remain unchanged. This means complete similitude would be achieved for any free surface hydrodynamic phenomena governed by the cylindrical form of the Navier-Stokes equations if the value of each dimensionless coefficient in Equations 23 through 26 remains constant between prototype and model. Note that all nondimensional terms without coefficients will be in similitude.

For steady flow, the only potential scale effects arise from the convective accelerations, and the only dimensionless coefficients are (X/Z) and its inverse. Therefore, the requirement for similitude of the convective accelerations is simply: 


$$
\left(\frac{X}{Z}\right)_{\text {prototype }}=\left(\frac{X}{Z}\right)_{\text {model }}
$$

or expressed in terms of scale ratios

$$
N_{X}=N_{Z}
$$

which means a geometrically undistorted model. From Equations 23 through 26 the following can be concluded:

convective acceleration terms in similitude

$$
v_{r} \frac{\partial v_{r}}{\partial r} ; \frac{v_{\theta}}{r} \frac{\partial v_{r}}{\partial \theta} ; \frac{v_{\theta}^{2}}{r} ; v_{r} \frac{\partial v_{\theta}}{\partial r} ; \frac{v_{\theta}}{r} \frac{\partial v_{\theta}}{\partial \theta} ; \frac{v_{r} v_{\theta}}{r} ; v_{z} \frac{\partial v_{z}}{\partial z}
$$

convective acceleration terms not in similitude

$$
v_{z} \frac{\partial v_{r}}{\partial z} ; v_{z} \frac{\partial v_{\theta}}{\partial z} ; v_{r} \frac{\partial v_{z}}{\partial r} ; \frac{v_{\theta}}{r} \frac{\partial v_{z}}{\partial \theta}
$$

The two nonsimilar convective accelerations contained in the horizontal direction momentum equations are larger in the model than they should be by a factor equal to the geometric distortion, i.e.,

$$
\Omega\left(\hat{v}_{z} \frac{\partial \hat{v}_{r}}{\partial \hat{z}} ; \hat{v}_{z} \frac{\partial \hat{v}_{\theta}}{\partial \hat{z}}\right)_{\text {proto }}=\left(\hat{v}_{z} \frac{\partial \hat{v}_{r}}{\partial \hat{z}} ; \hat{v}_{z} \frac{\partial \hat{v}_{\theta}}{\partial \hat{z}}\right)_{\text {model }}
$$

where $\Omega=N_{x} / N_{z}$. This implies that horizontal convective accelerations due to vertical gradients of the radial and tangential velocities $\left(v_{r}, v_{\theta}\right)$ are greater in the distorted model than in the prototype.

Conversely, the two nonsimilar convective accelerations contained in the vertical momentum equation are smaller in the model than they should be by a factor equal to the inverse of the geometric distortion, i.e.,

$$
\frac{1}{\Omega}\left(\hat{v}_{r} \frac{\partial \hat{v}_{z}}{\partial \hat{r}} ; \frac{\hat{v}_{\theta}}{\hat{r}} \frac{\partial \hat{v}_{z}}{\partial \hat{\theta}}\right)_{\text {proto }}=\left(\hat{v}_{r} \frac{\partial \hat{v}_{z}}{\partial \hat{r}} ; \frac{\hat{v}_{\theta}}{\hat{r}} \frac{\partial \hat{v}_{z}}{\partial \hat{\theta}}\right)_{\text {model }}
$$


In this case the vertical convective accelerations due to horizontal gradients of the vertical velocity $\left(v_{z}\right)$ are less in the distorted model than in the prototype.

It is difficult to assess definitively the impact of the nonsimilar convective acceleration terms in bend flows. Thorne and Abt (1993) overviewed numerical modeling approaches based on the cylindrical form of the Navier-Stokes equations. A basic assumption is that vertical velocities in the uniform flow are small, allowing the use of depth-integrated flow equations. This assumption implies that the nonsimilar convective accelerations of Equation 32 are also negligible.

Thorne and Abt (1993) discussed two major schools of thought regarding the horizontal convective accelerations. Early work by Engelund (1974) argued that in the cross-stream (r-direction) momentum equation the centrifugal acceleration $\left(v_{\theta}{ }^{2} / r\right)$ is balanced by the pressure gradient and cross-stream bottom shear stress. All other convective accelerations are negligible. In the downstream ( $\theta$-direction) momentum equation the water-surface slope is balanced only by downstream bed shear stress, thus ignoring any influence of convective accelerations. If Engelund's simplifying assumptions are reasonable, then correct similitude of bottom friction will be far more important in a distorted model than the dissimilar convective acceleration terms.

The second school of thought maintains that the horizontally directed convective accelerations are important, but recognizes very severe field requirements for validating numerical models because it requires measuring cross-channel water-surface elevations to millimeter accuracy (Thorne and Abt 1993). However, even in this case the emphasis is placed on those horizontal convective accelerations which are shown to be in similitude in a distorted physical model. Because of the assumption that vertical velocities are small, the nonsimilar convective accelerations given in Equation 4-31 must also be considered negligible in the numerical model development. Thorne and Abt (1993) recommended adopting models following the thesis of Engelund (1974) because they have been shown to give reasonable results.

A geometrically distorted physical model with proper attention to bottom surface roughness includes more of the physics than depth-integrated numerical models despite having convective acceleration terms that exhibit a scale effect. The four convective accelerations that contain a scale effect in a distorted physical model of river bends are considered inconsequential in most practical numerical modeling. This provides some level of comfort in using a distorted physical model to simulate flow around bends. 


\section{Turbulence Scale Effect Experiments}

\section{Introduction}

A series of flow experiments was conducted using the CHL precision flow table described in Chapter 2. The objective of these systematic tests was to evaluate the turbulence scale effect in geometrically distorted physical models for specific situations where flow turbulence is caused by solid boundaries. Four distinct flow situations were considered and discussed in the following paragraphs.

\section{Case 1: Unconstrained flow separation at vertical edge}

These experiments reproduced flow separation and downstream entrainment that occurs in the lee of a protrusion placed at right angle to the flow as illustrated in Figure 18(a). The edge of the protrusion was vertical, and downstream the jet expansion was unhindered by any boundaries. This configuration is typical of the downstream edge of man-made structures such as retaining walls that extend a considerable distance into the flow. It was expected this configuration would produce horizontal turbulence components that are much more pronounced than the vertical components.

\section{Case 2: Constrained flow separation at vertical edge}

These tests were virtually identical with the unconstrained flow separation with the only difference being the flow-parallel boundaries downstream of flow separation as illustrated by Figure 18(b). The intent was to gauge the influence of these boundaries on flow entrainment.

\section{Case 3: Flow separation at sloping edge}

These experiments duplicated the conditions of unconstrained flow separation at a vertical edge except the edge was sloped as represented by the sketch of Figure 18(c). Gap distance between the two walls is closest at the toe, and the opening width increases toward the water surface. Turbulence generated by flow separation was expected to have horizontal and vertical components with similar magnitudes, so a scale effect was anticipated. 


\section{Case 4: Flow separation at vertical step}

Figure 18(d) shows flow over a vertical step. Flow separation that occurs in the lee of the step exhibits a dominant vertical turbulence component. Although this is a less common situation in the real world, the experiments provided an opportunity to evaluate the scale effect where vertical turbulence generation is predominant.

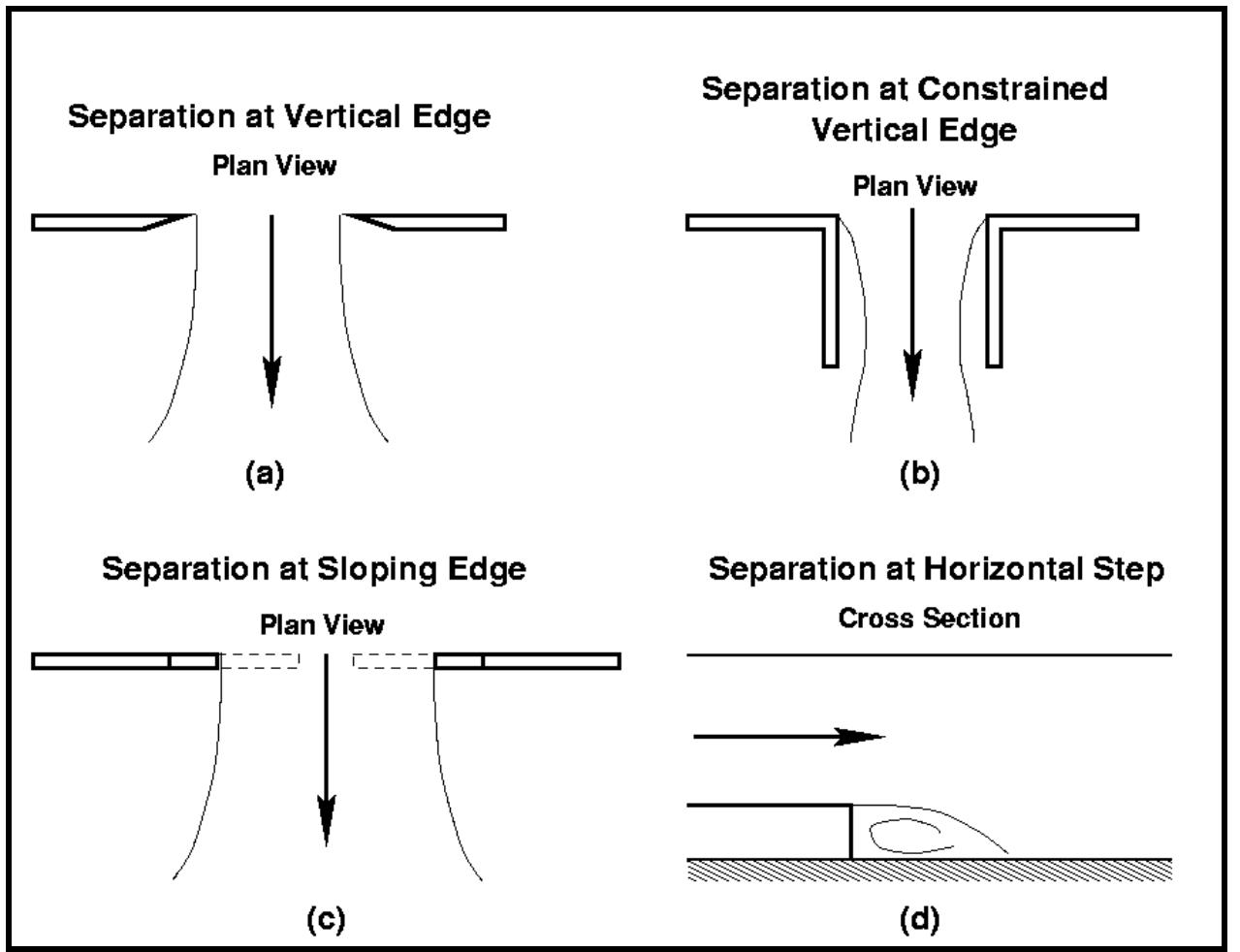

Figure 18. Turbulent flow cases tested on flow table

For each different configuration the first test in the series was considered to be the target or prototype condition representing results from an undistorted model. Subsequent tests in the series were designed to be increasingly distorted versions of the target condition. In other words, the follow-up tests were distorted scale models of the first test.

Velocity measurements using the laser Doppler velocimeter were obtained throughout the area of interest including the turbulent regions created by the flow disturbance. Measured mean values of the two horizontal components of velocity at all locations for each of the distorted tests were scaled to the undistorted target equivalent by applying appropriate length scale factors. These scaled horizontal velocity vectors were then compared to those obtained for the target condition. Good correspondence indicated minimal turbulence scale effect.

\section{Scale relationships}

The geometrically scaled versions of each target condition were designed following established Froude scaling relationships for distorted flow models. 
Prototype-to-model scale ratios for horizontal lengths, vertical lengths, flow velocity, and total discharge are given in Table 4.

\begin{tabular}{|c|c|c|c|}
\hline \multicolumn{4}{|c|}{\begin{tabular}{|l} 
Table 4 \\
Distorted Model Froude Scales
\end{tabular}} \\
\hline Scale & Ratio & Notation & Relationship \\
\hline Horizontal length & $x_{p} / x_{m}$ & $N_{X}$ & - \\
\hline Vertical length & $z_{p} / Z_{m}$ & $N_{Z}$ & - \\
\hline Model distortion & $N_{X} / N_{Z}$ & $\Omega$ & - \\
\hline Velocity & $v_{p} / v_{m}$ & $N_{V}$ & $N_{V}=N_{Z}^{1 / 2}$ \\
\hline Discharge & $Q_{p} / Q_{m}$ & $N_{Q}$ & $N_{Q}=N_{X}: N_{Z}{ }^{3 / 2}=\Omega: N_{Z}{ }^{5 / 2}$ \\
\hline
\end{tabular}

Model scale was manipulated by specifying appropriate horizontal and vertical length scales and the discharge scale. Two of the scales can be selected, and the third is then constrained by the scale equations. For most test series it was more convenient to keep the flow discharge constant for all experiments and change the horizontal and vertical dimensions to suit the different model distortions. This way the flow valve remained at the same setting and difficulties in adjusting the flow rate to a precise value were avoided.

Solving the discharge scale equation for $N_{z}$ gives:

$$
N_{Z}=\left(\frac{N_{Q}}{\Omega}\right)^{2 / 5}
$$

For a given distortion, $\Omega$, and a specified discharge scale (usually $N_{Q}=1$ ), the vertical length scale is determined, and the horizontal length scale is found as:

$$
N_{X}=\Omega N_{Z}
$$

Once the scale ratios were determined, horizontal and vertical dimensions in the distorted model could be determined based on the prototype target dimensions. For constant discharge, water depths increased with distortion while the gap distance decreased.

\section{Velocity measurements}

For each test series a velocity measurement grid was established. The grid encompassed the primary region of interest and included points within the turbulent and nonturbulent portions of the flow regime. The uniform, rectangular grid positions were entered into the computer program, which controlled the traversing system and acquired the data. Grid dimensions established for the target condition were scaled appropriately for each distorted test so the 
measurement positions corresponded. The laser Doppler velocimeter probe was adjusted vertically to an elevation in the water column specified for each experiment. For example, in some test cases the vertical elevation was middepth, so in a distorted version of the experiment the water depth increased, and the probe position needed to be raised.

Before commencing measurements, a box was placed around the laser beams to create a condition of zero flow velocity. Measurements were then taken to establish "offsets" in the system due to local temperature effects on the analogto-digital converter cards. These values were recorded in the experiment log, and the offset test was repeated at completion of the experiment to see if any significant drift in the electronics had occurred during the test. If the difference was not too great, an average was taken, and the offsets were applied to the measured time series of turbulent velocity fluctuations. Greater variation in the offsets necessitated repeating the test.

For each test, flow was initiated on the flow table and sufficient time was allowed for the flow to reach a quasi-steady state condition before beginning data acquisition. The laser Doppler system was programmed to record two components of horizontal velocity at each grid point. Time series data of velocity fluctuations were collected for $10 \mathrm{sec}$ at a rate of $100 \mathrm{~Hz}$ per channel. At completion, the traverse moved to the next point, waited by $0.5 \mathrm{sec}$ for movement vibrations to dampen, then repeated the process. At the end of the experiment, data were converted to engineering units and moved to a more modern computer system for subsequent analysis and plotting.

Inherent in this measurement scheme is the assumption of quasi-steady flow conditions. Previous experiments with this facility had shown that the timeaveraged flow components in the nonturbulent portion were reproducible. Initial testing at the start of this project examined the duration of sampling needed at a point to get a reasonably stable mean value, and $10 \mathrm{sec}$ was found to be well above the threshold necessary. However, in the far field well away from the exit structure, slowly oscillating, large-scale gyres develop which would require a much longer sampling duration to establish a repeatable mean value.

Before each experiment, the surfaces of the flow table glass bottom were cleaned of minute particles that might interfere with the laser beams. However, sometimes a small particle in the flow would deposit at a grid location and partially block one of the laser beams. When this happened, either no data were acquired, or the measured data were significantly smaller than actual. Where measurement "dropouts" occurred, it was usually obvious in the vector plots because all the surrounding values were uniformly larger.

The following sections provide details about each of the test series conducted. Representative results and comparisons are given. Complete results, mostly in graphical form, are included in Appendix A, Appendix B, and Appendix C.

\section{Case 1: Flow Separation at Vertical Edge - Free Jet}

The first set of tests was performed on a free jet constrained by a vertical boundary with a sharp edge as illustrated in Figure 18(a). In this scenario, it was expected that only strong horizontal turbulence components would be generated, 
and the dominant turbulence terms would be those shown to be in similitude in the previous section.

After a prototype test case was established and the velocity field measured, different distorted models of the prototype case were also tested and the velocities compared. The relative distortion from prototype can be seen in Figure 19. Scaling factors for each test in the series along with relevant values for discharge and key horizontal $\left(X_{p}, X_{m}\right)$ and vertical $\left(Z_{p}, Z_{m}\right)$ dimensions are listed in Table 5.

\section{Experiment setup}

The planform layout of the experiment on the flow table is sketched in Figure 20. The prototype consisted of a $300-\mathrm{mm}$ (11.8 in.) gap with vertical walls having sharp edges cut at a 30 deg angle. Water depth was set at $40 \mathrm{~mm}$ (1.6 in.) and a uniform flow of $1.5 \mathrm{~L} / \mathrm{sec}(3.17 \mathrm{pints} / \mathrm{sec})$ was established. This flow rate provided an average velocity over the gap cross section of $0.125 \mathrm{~m} / \mathrm{sec}$ $(0.41 \mathrm{ft} / \mathrm{sec})$. Velocities were measured at mid-depth in a domain directly in front of the gap. The measurement area (shown shaded on Figure 20) had a width that was about 120 percent of the gap width and length extending about two-thirds of the gap width downstream.

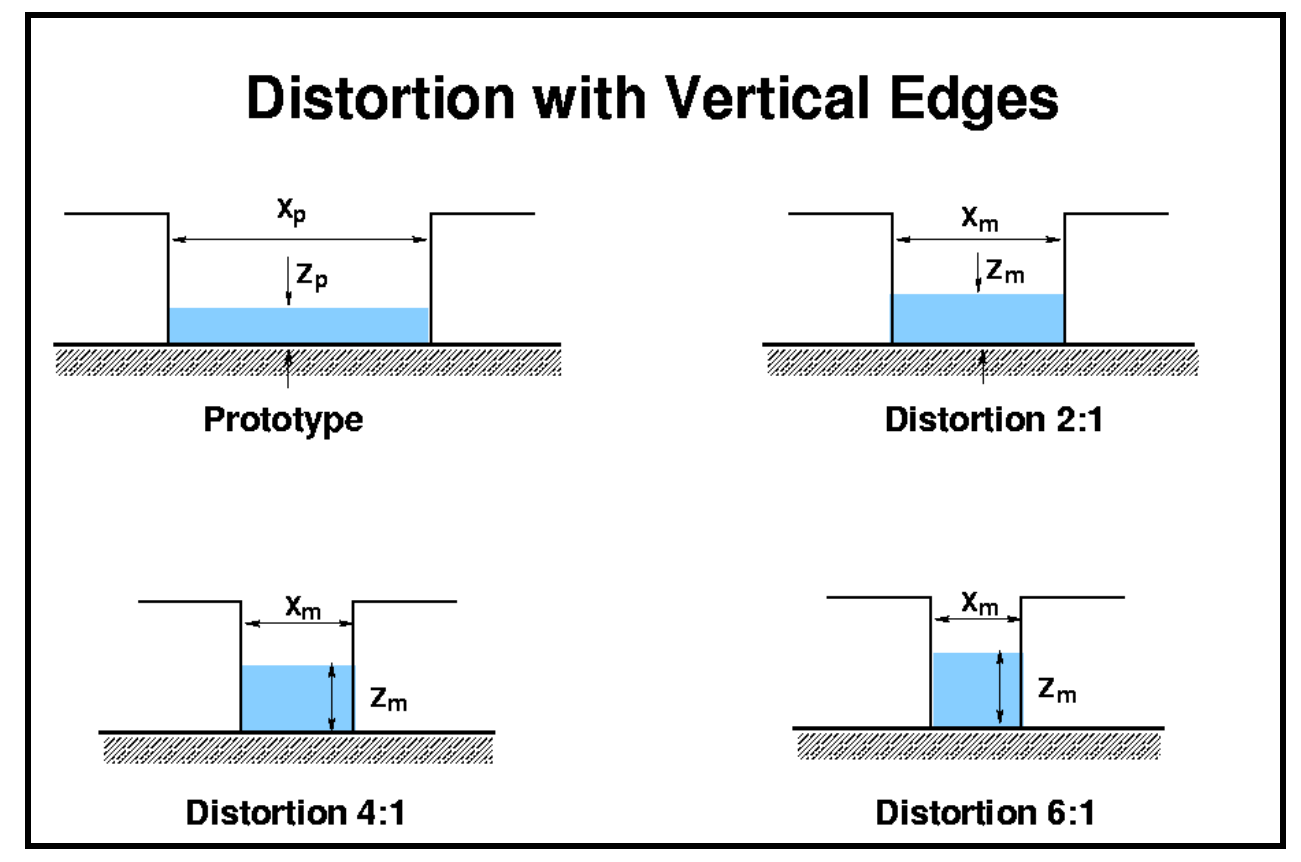

Figure 19. Cross sections showing distortion for vertical edge tests 


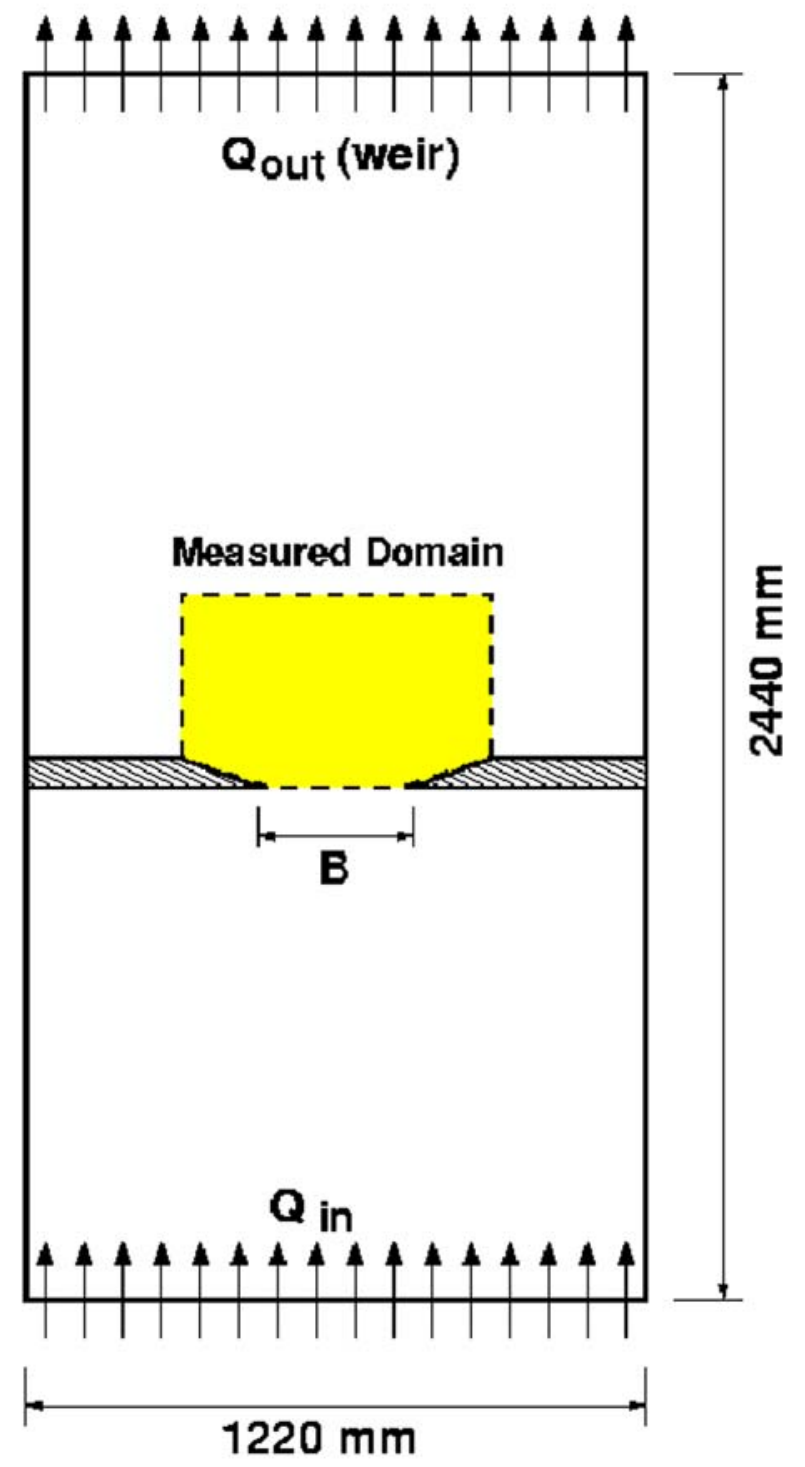

Figure 20. Experiment setup for Case 1 free jet flow separation at vertical edge

The prototype case, and scaled models of the prototype having distortions ratios $\left(N_{X} / N_{z}\right)$ equal to 2,4 , and 6 , were tested for three different flow rates $(Q=1.5 \mathrm{~L} / \mathrm{sec}, 1.0 \mathrm{~L} / \mathrm{sec}$, and $0.75 \mathrm{~L} / \mathrm{sec})$. This gave a total of 12 experiments, as detailed in Table 5. The elevation of the LDV measurement volume above the bottom, and the horizontal measurement grid spacing $(\Delta x, \Delta y)$ for each experiment are listed in Table 6. 


\begin{tabular}{|c|c|c|c|c|c|c|c|c|c|c|}
\hline \multicolumn{11}{|c|}{$\begin{array}{l}\text { Table } 5 \\
\text { Distortion Experiment Scale Factors and Parameters }\end{array}$} \\
\hline Tests & $N_{X} / N_{Z}$ & $N_{Q}$ & $N_{X}$ & $N_{Z}$ & $\begin{array}{l}Q_{p} \\
(\mathrm{~L} / \mathrm{sec})\end{array}$ & $\begin{array}{l}Q_{m} \\
(\mathrm{~L} / \mathrm{sec})\end{array}$ & $\begin{array}{l}X_{p} \\
(\mathrm{~mm})\end{array}$ & $\begin{array}{l}X_{m} \\
(\mathrm{~mm})\end{array}$ & $\underset{p}{Z_{p}}$ & $\begin{array}{l}Z_{m} \\
(\mathrm{~mm})\end{array}$ \\
\hline 1 & 1 & 1.0 & 1.000 & 1.000 & 1.5 & 1.5 & 300 & 300 & 40 & 40 \\
\hline 2 & 2 & 1.0 & 1.516 & 0.758 & 1.5 & 1.5 & 300 & 198 & 40 & 53 \\
\hline 3 & 4 & 1.0 & 2.297 & 0.574 & 1.5 & 1.5 & 300 & 131 & 40 & 70 \\
\hline 4 & 6 & 1.0 & 2.930 & 0.488 & 1.5 & 1.5 & 300 & 102 & 40 & 82 \\
\hline 5 & 1 & 1.5 & 1.176 & 1.176 & 1.5 & 1.0 & 300 & 255 & 40 & 34 \\
\hline 6 & 2 & 1.5 & 1.783 & 0.891 & 1.5 & 1.0 & 300 & 168 & 40 & 45 \\
\hline 7 & 4 & 1.5 & 2.702 & 0.675 & 1.5 & 1.0 & 300 & 111 & 40 & 59 \\
\hline 8 & 6 & 1.5 & 3.446 & 0.547 & 1.5 & 1.0 & 300 & 87 & 40 & 70 \\
\hline 9 & 1 & 2.0 & 1.320 & 1.320 & 1.5 & 0.75 & 300 & 227 & 40 & 30 \\
\hline 10 & 2 & 2.0 & 2.000 & 1.000 & 1.5 & 0.75 & 300 & 150 & 40 & 40 \\
\hline 11 & 4 & 2.0 & 3.031 & 0.758 & 1.5 & 0.75 & 300 & 99 & 40 & 53 \\
\hline 12 & 6 & 2.0 & 3.866 & 0.644 & 1.5 & 0.75 & 300 & 78 & 40 & 62 \\
\hline
\end{tabular}

\begin{tabular}{|c|c|c|c|c|}
\hline \multicolumn{5}{|c|}{$\begin{array}{l}\text { Table } 6 \\
\text { Measurement Geometry of Vertical Edge Tests }\end{array}$} \\
\hline Tests & $N_{X} / N_{Z}$ & $\begin{array}{l}\Delta x \\
(\mathrm{~mm})\end{array}$ & $\begin{array}{l}\Delta y \\
(\mathrm{~mm})\end{array}$ & $\begin{array}{l}\begin{array}{l}\text { Vertical Position } \\
\text { (mm above bottom) }\end{array} \\
\end{array}$ \\
\hline 1 & 1 & 12 & 21 & 25 \\
\hline 2 & 2 & 7.9 & 13.86 & 33 \\
\hline 3 & 4 & 5.2 & 9.15 & 43 \\
\hline 4 & 6 & 4.1 & 7.17 & 51 \\
\hline 5 & 1 & 10.2 & 17.86 & 21 \\
\hline 6 & 2 & 6.73 & 11.78 & 27.5 \\
\hline 7 & 4 & 4.44 & 7.77 & 38 \\
\hline 8 & 6 & 3.48 & 6.09 & 43.75 \\
\hline 9 & 1 & 9.1 & 15.91 & 19 \\
\hline 10 & 2 & 6 & 10.5 & 25 \\
\hline 11 & 4 & 3.96 & 6.93 & 33 \\
\hline 12 & 6 & 3.1 & 5.43 & 38 \\
\hline
\end{tabular}




\section{Case 1 results}

Graphical results obtained from the four tests conducted with a discharge scale of $N_{Q}=1.5$ are presented here as representative of this test configuration. Complete graphical results for all 12 tests are included in Appendix A.

Measured velocity vectors for the prototype case are shown in Figure 21, and the corresponding results from models with distortion 2,4 , and 6 are shown scaled up to prototype in Figures 22, 23, and 24, respectively. Scaling was performed using the scale factors presented in Table 5. A similar distinct jet flow with low velocity entrainment adjacent to the jet is seen in all the velocity vector plots.

Comparison between prototype and distorted models was done visually by superimposing vector diagrams from two experiments. Figure 25 plots the vector fields from the prototype and the distortion 6 model tests at actual scale to give an idea of the horizontal length scale difference between the two experiments. A similar comparison with distorted model results scaled to prototype size is shown in Figure 26 with the distorted model results displaced slightly to the right of the prototype results. As seen, the comparison between prototype and distorted model is favorable, even in the slow-moving flow entrainment region; and current patterns seem to be reproduced well in this configuration despite the presence of strong horizontal turbulence in the flow. Similar good agreement was found for comparisons between prototype and all distorted models at all flow rates as shown by the plots in Appendix A.

The ratio between prototype and scaled-up velocity components was determined at each measured point in the flow. Ratios of the cross-flow velocity components for experiments with discharge scale $N_{Q}=1.5$ are presented in Figure 27, and the corresponding ratios in the principal flow direction are plotted in Figure 28. Perfect correspondence would be represented by horizontal lines with no deviations.

The ratio plots enable recognition of spatial positions where similitude might be in question. Variations shown in the crossflow direction (Figure 27) are likely caused by taking the ratio of very small velocities; and consequently, much of the variation could be measurement inaccuracy. Good similitude is seen in the principal flow direction as illustrated in Figure 28 where the only significant deviation appears near the boundary of the jet and ambient fluid. Some of this variation might be caused by measuring at slightly different positions along the jet boundary where there is a steep velocity gradient. Given the amount of flow turbulence generated at the jet boundary, these experiments indicated good similitude existed between the prototype and distorted models. Similar comparison plots for the other Case 1 experiments are in Appendix A. 


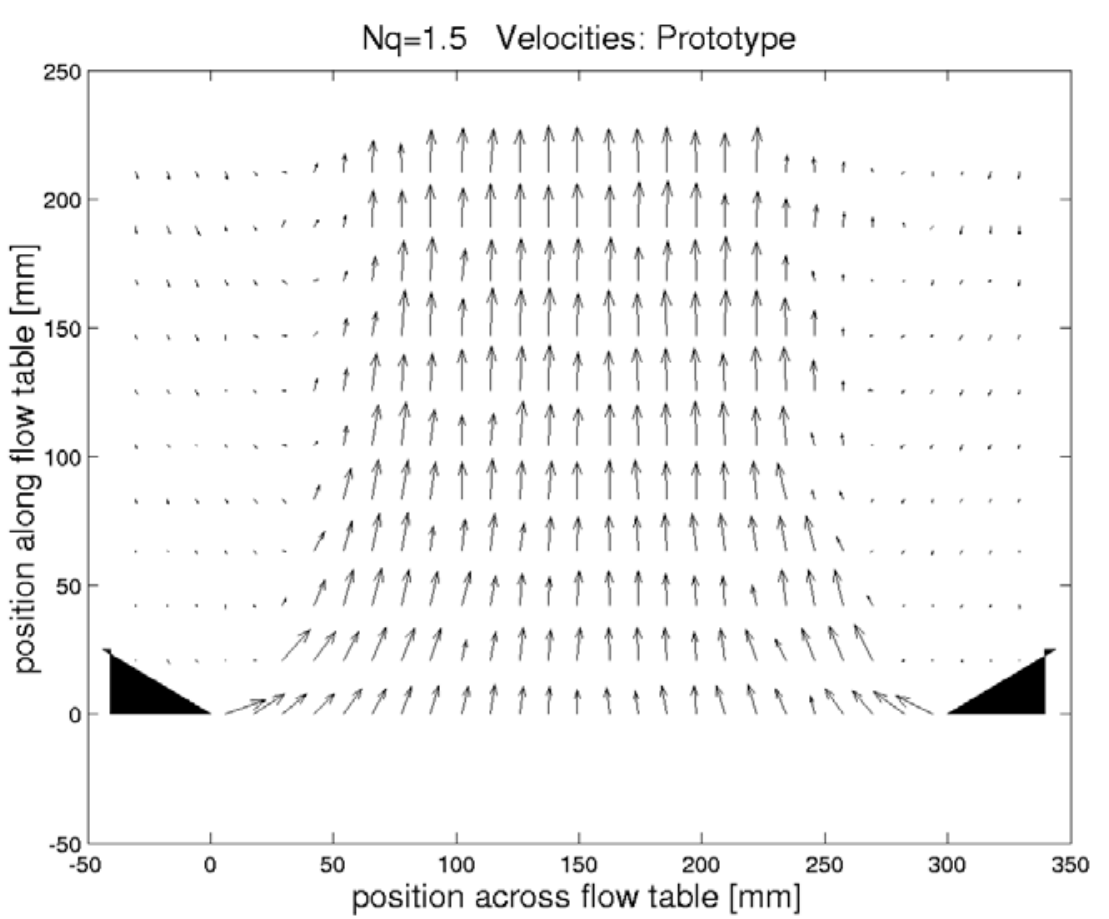

Figure 21. Velocity field $N_{Q}=1.5$, prototype (Case 1)

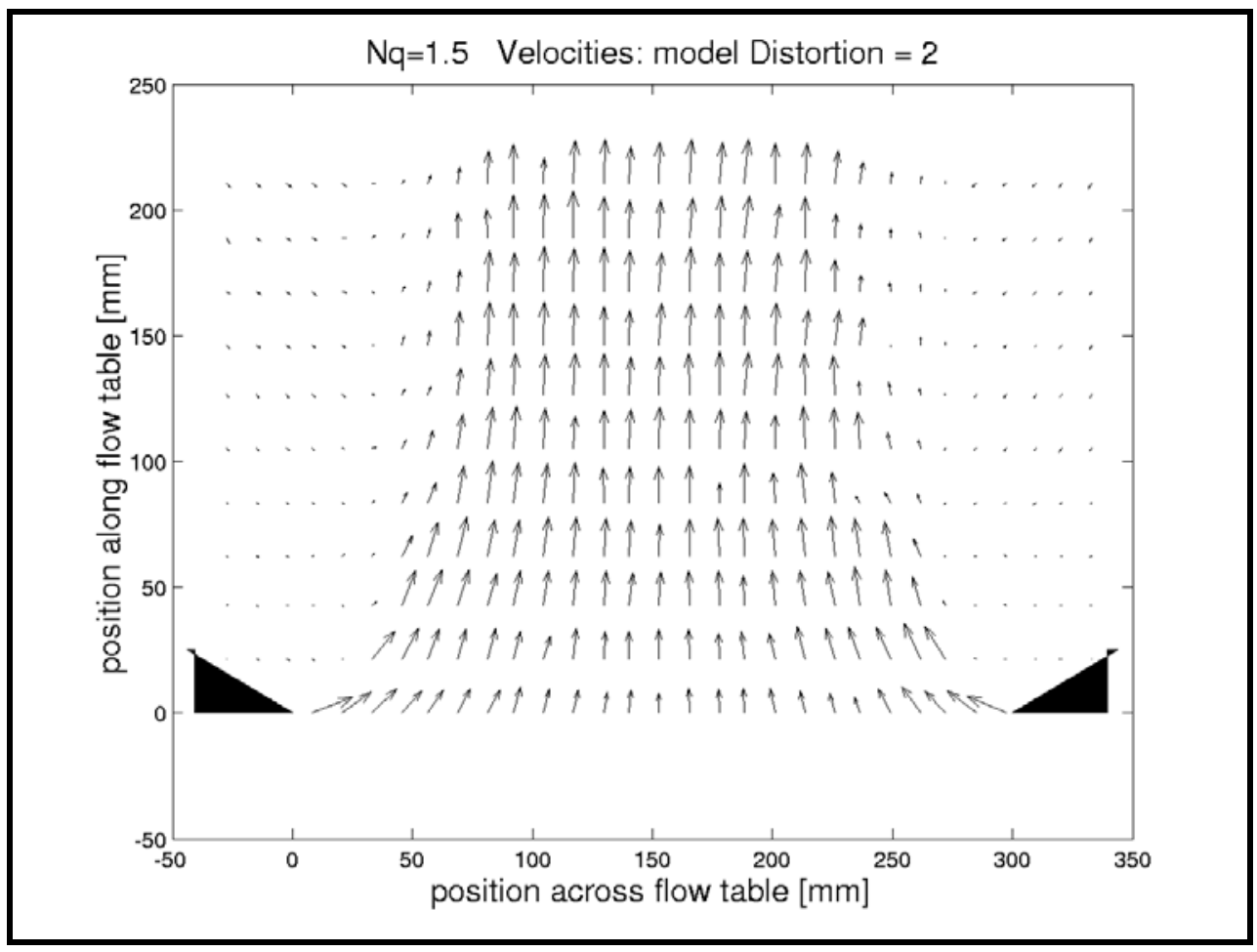

Figure 22. Velocity field $N_{Q}=1.5$, distortion $=2($ Case 1$)$ 


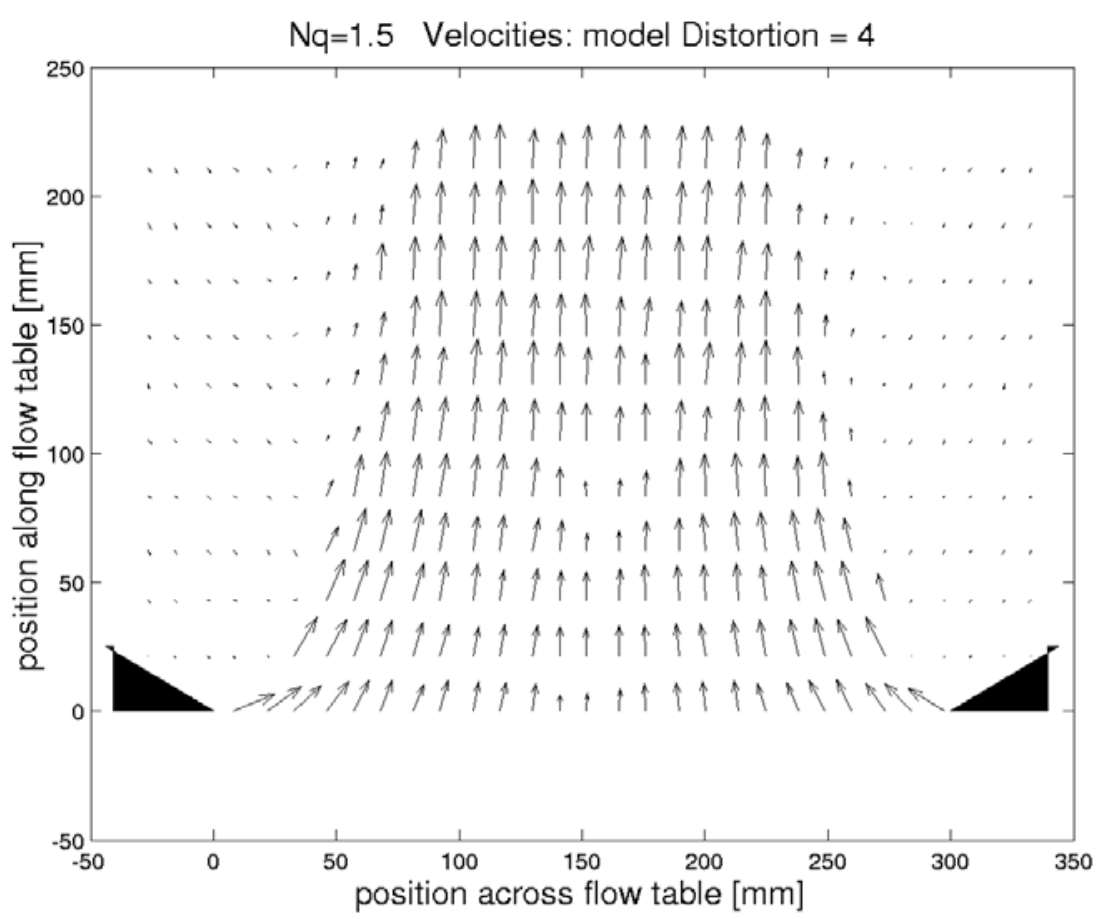

Figure 23. Velocity field $N_{Q}=1.5$, distortion $=4$ (Case 1)

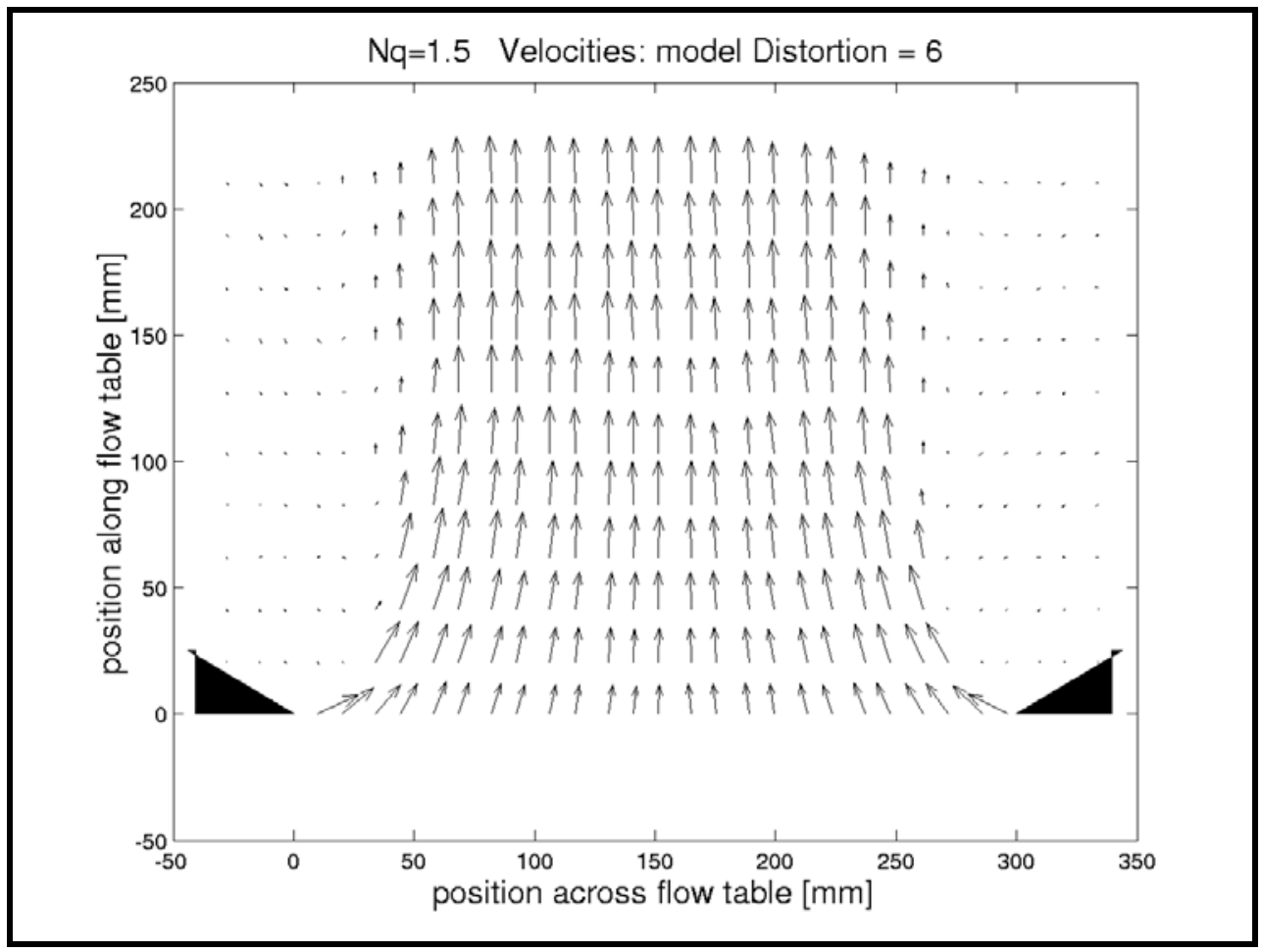

Figure 24. Velocity field $N_{Q}=1.5$, distortion $=6($ Case 1$)$ 


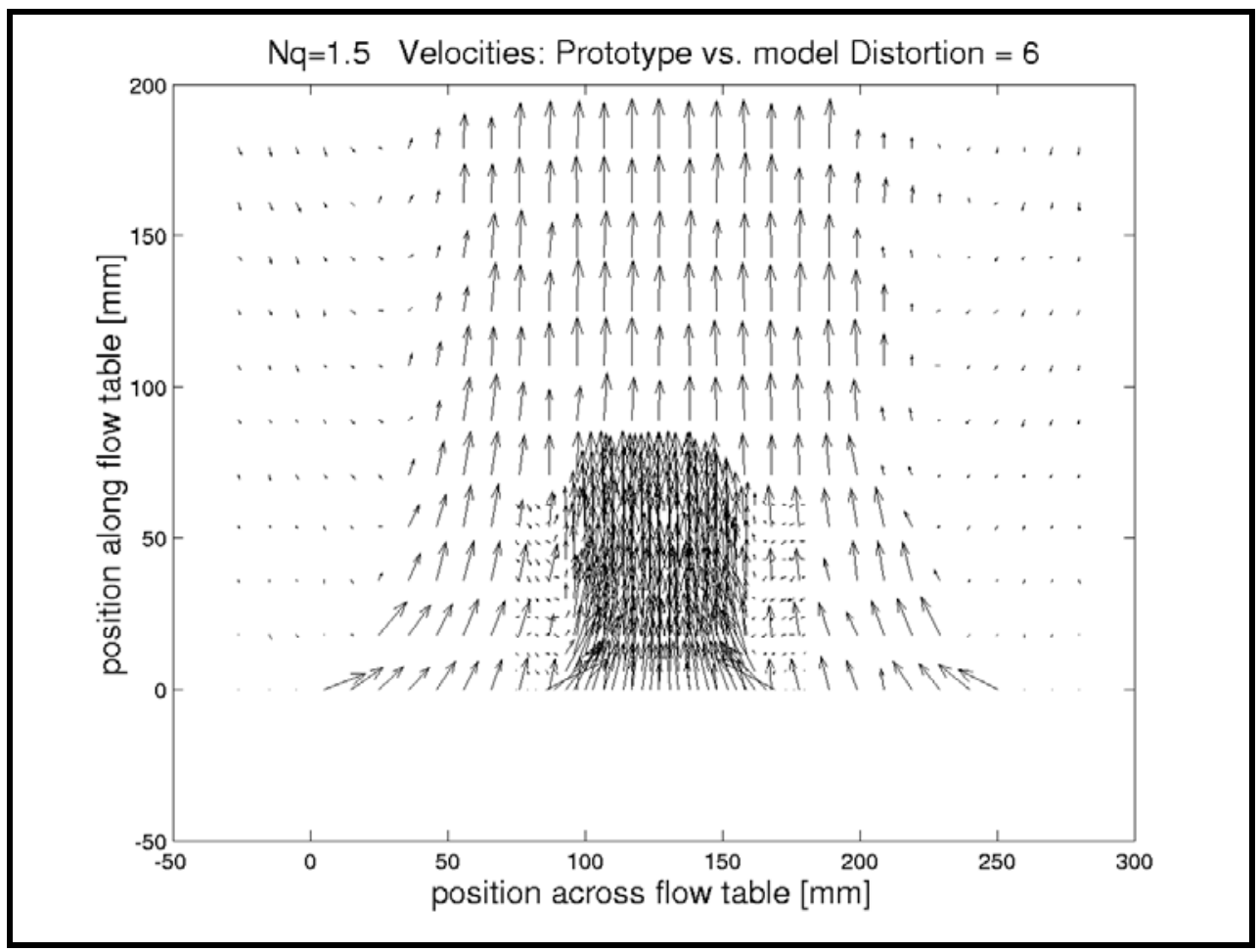

Figure 25. Comparison between prototype and distortion $=6, N_{Q}=1.5$ (Case 1)

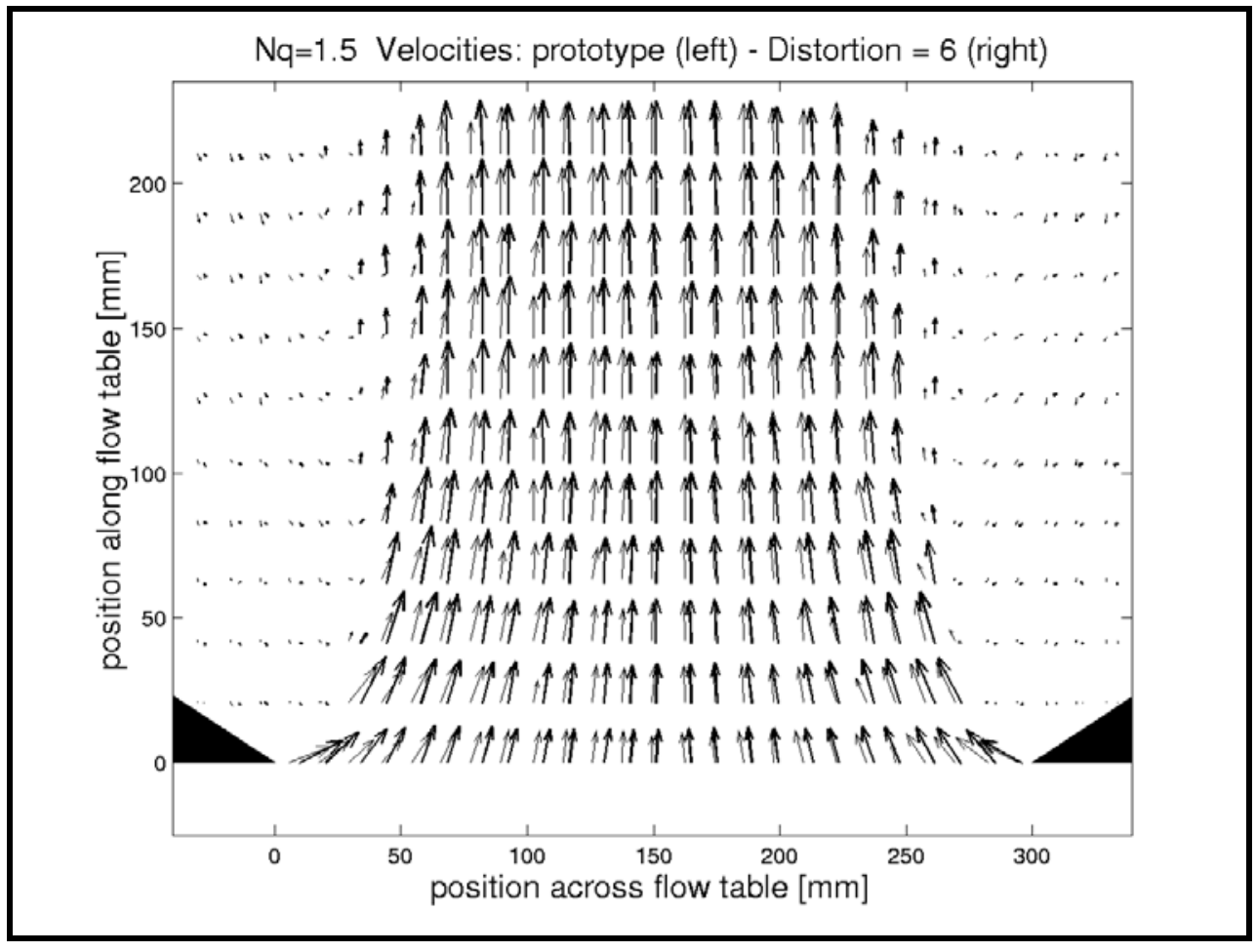

Figure 26. Velocity field $N_{Q}=1.5$, prototype vs. distortion $=6$ (Case 1$)$ 


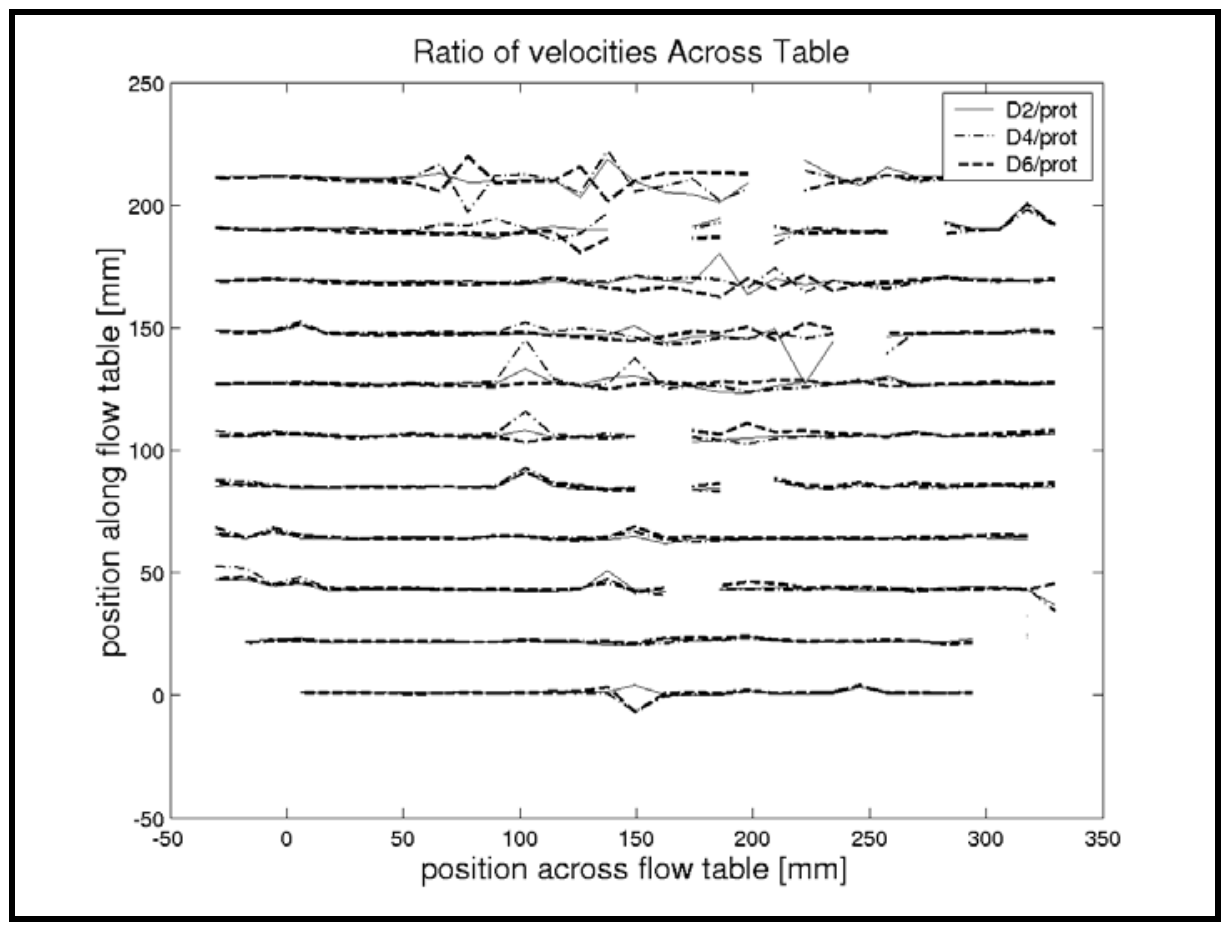

Figure 27. Crossflow velocity ratios, distortion $=2,4,6$ over prototype $\left(N_{Q}=1.5\right.$, Case 1)

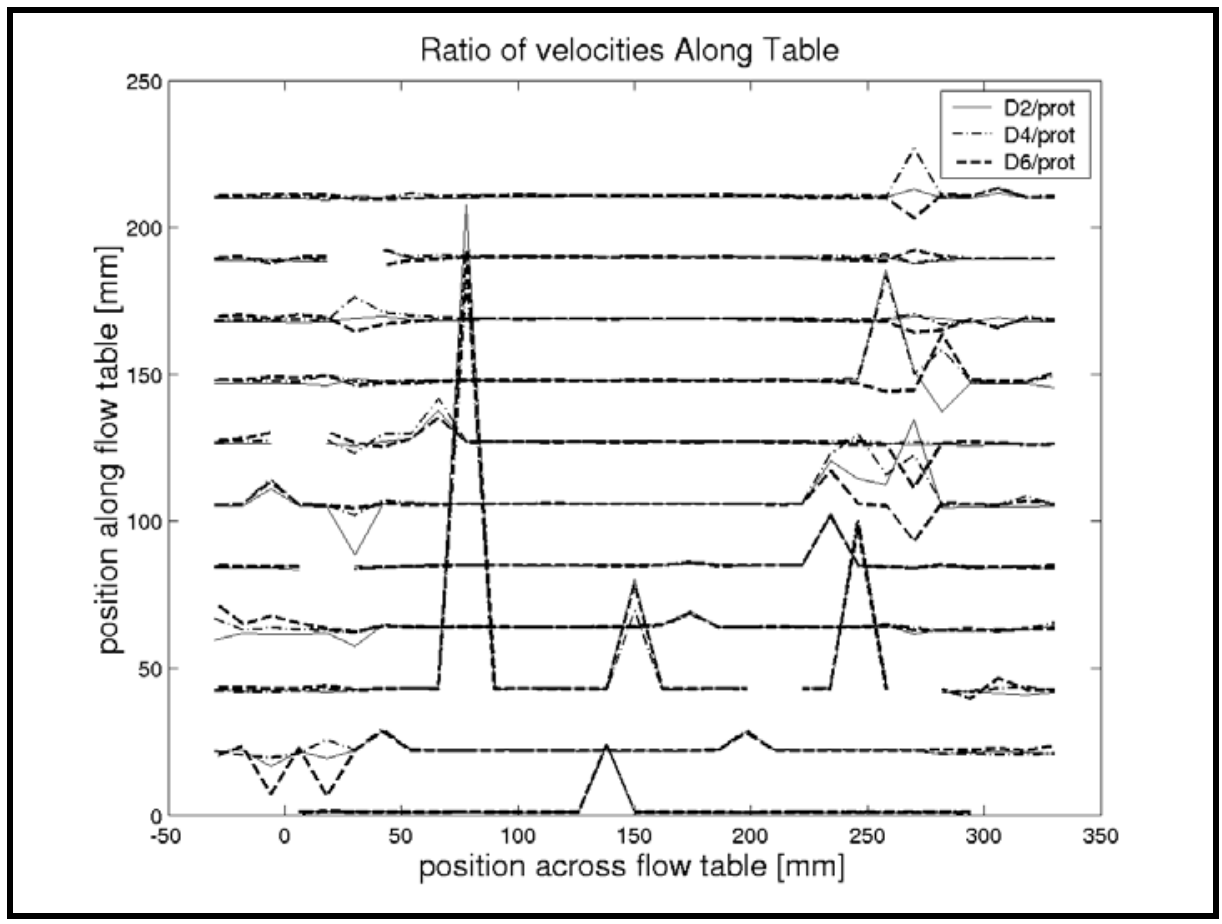

Figure 28. Principal flow direction velocity ratios, distortion $=2,4,6$ over prototype $\left(N_{Q}=1.5\right.$, Case 1) 


\section{Case 1 discussion and conclusions}

Comparison between measured velocities from the prototype and velocities from the distorted models scaled up to prototype for Case 1 indicated that major flow patterns and velocity vectors were in good similitude. Differences between measured velocity vectors could be ascribed to small perturbations in the steadiness of the flow, to small errors in the location of the measurement, or to variations of the velocity offset measured by the LDV apparatus over the course of the measurements. In addition, it was seen that small errors in the geometry and orientation of the gap and solid boundaries could affect the jet geometry, so care was taken to align the boundaries carefully for each experiment.

Results measured in the Case 1 tests indicated that there were no substantial differences between geometrically undistorted and distorted physical flow models when the flow turbulence was manifested primarily in the horizontal plane with only weak vertical turbulent fluctuations. The observation conforms with the theoretical analysis given in Chapter 4.

\section{Case 2: Flow Separation at Vertical Edge - Constrained Jet}

The Case 2 experiments were virtually identical to the Case 1 free jet tests, with the only difference being the two parallel boundaries downstream of the gap that served to constrain jet expansion as shown in Figure 18(b). The pair of jetties parallel to the principal flow direction extended for one-half the gap width, and the ratio of gap width to jetty length was maintained in the distorted model versions.

\section{Experiment setup}

As in Case 1, measurements were first made using a configuration that was assumed to be the prototype. Subsequent experiments used geometrically distorted versions of the prototype as illustrated by the sketch in Figure 19. Scaling factors for each test in the series along with relevant values for discharge and key horizontal $\left(X_{p}, X_{m}\right)$ and vertical $\left(Z_{p}, Z_{m}\right)$ dimensions were the same as Case 1 and are listed in Table 5. The planform layout of the experiment on the flow table is sketched in Figure 29, and the measurement area had a width equal to the gap width and length extending two-thirds of the gap width downstream past the end of the jetties.

The prototype case and scaled models of the prototype having distortions ratios $\left(N_{X} / N_{Z}\right)$ equal to 2,4 , and 6 , were tested for three different flow rates $(Q=1.5 \mathrm{~L} / \mathrm{sec}, 1.0 \mathrm{~L} / \mathrm{sec}$, and $0.75 \mathrm{~L} / \mathrm{sec})$. This gave a total of 12 experiments, as detailed in Table 5. The elevation of the LDV measurement volume above the bottom, and the horizontal measurement grid spacing $(\Delta x, \Delta y)$ for each experiment was the same as listed in Table 6 for the Case 1 tests. 


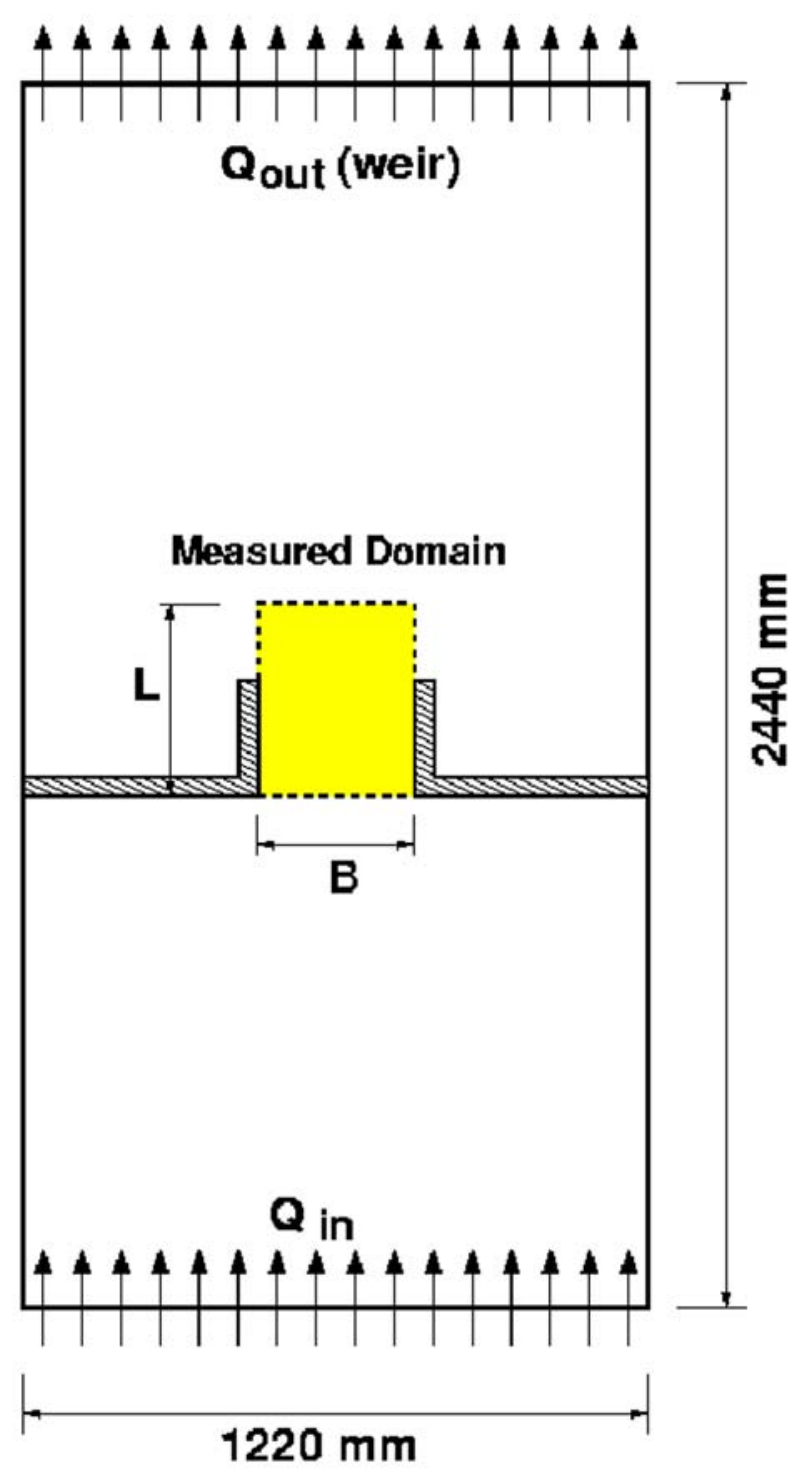

Figure 29. Experiment setup for Case 2 constrained jet flow separation at vertical edge

\section{Case 2 results}

Because Case 2 was similar to Case 1, only a few plots are shown in this section. Graphical results obtained from the four tests conducted with a discharge scale of $N_{Q}=1.0$ were selected as representative of this test configuration. Complete graphical results for all 12 tests are included in Appendix B.

Figure 30 compares the prototype velocity vector field with the vectors from the experiment with distortion of six scaled up to prototype size. The distorted model results were displaced slightly to the right of the prototype results. Once again, the comparison between prototype and distorted model was favorable, although there was some variation evident in the slow-moving flow entrainment region. Nevertheless, current patterns seemed to be reproduced well in this 
configuration despite the presence of strong horizontal turbulence in the flow. The influence of the flow-parallel jetties appeared to be minor. Similar good agreement was found for comparisons between prototype and all distorted models at all flow rates as shown by the plots in Appendix B.

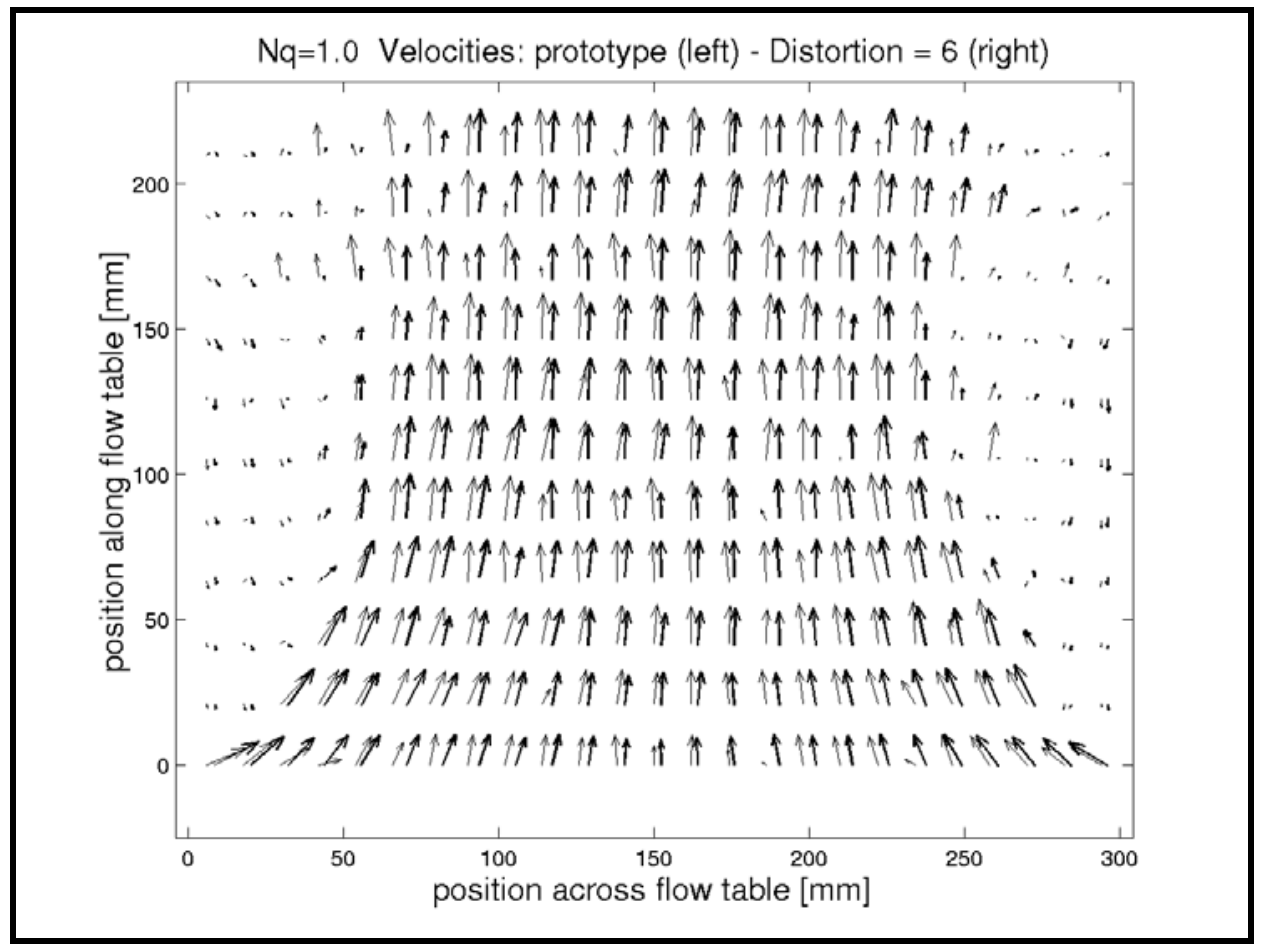

Figure 30 . Velocity field $N_{Q}=1.0$, prototype vs. distortion $=6($ Case 2$)$

Ratios of the crossflow velocity components for experiments with discharge scale $N_{Q}=1.0$ are presented in Figure 31, and the corresponding ratios in the principal flow direction are plotted on Figure 32. Results are similar to those found for the Case 1 experiments, and the same reasons for variation stated for Case 1 also apply for Case 2.

\section{Case 2 discussion and conclusions}

Case 2 experiments showed very similar behavior to the Case 1 tests, as should be expected. The overall flow distribution was very similar between prototype, and the distorted models indicated negligible scale effects related to generation of turbulence by vertical edges. It was concluded that good correspondence between prototype and distorted model indicated that the nonsimilar turbulence terms containing vertical turbulent fluctuations were small compared to the terms that contained squares and products of horizontal turbulent fluctuations. This means the turbulent fluctuations in the vertical direction were weak compared to the horizontal fluctuations. 


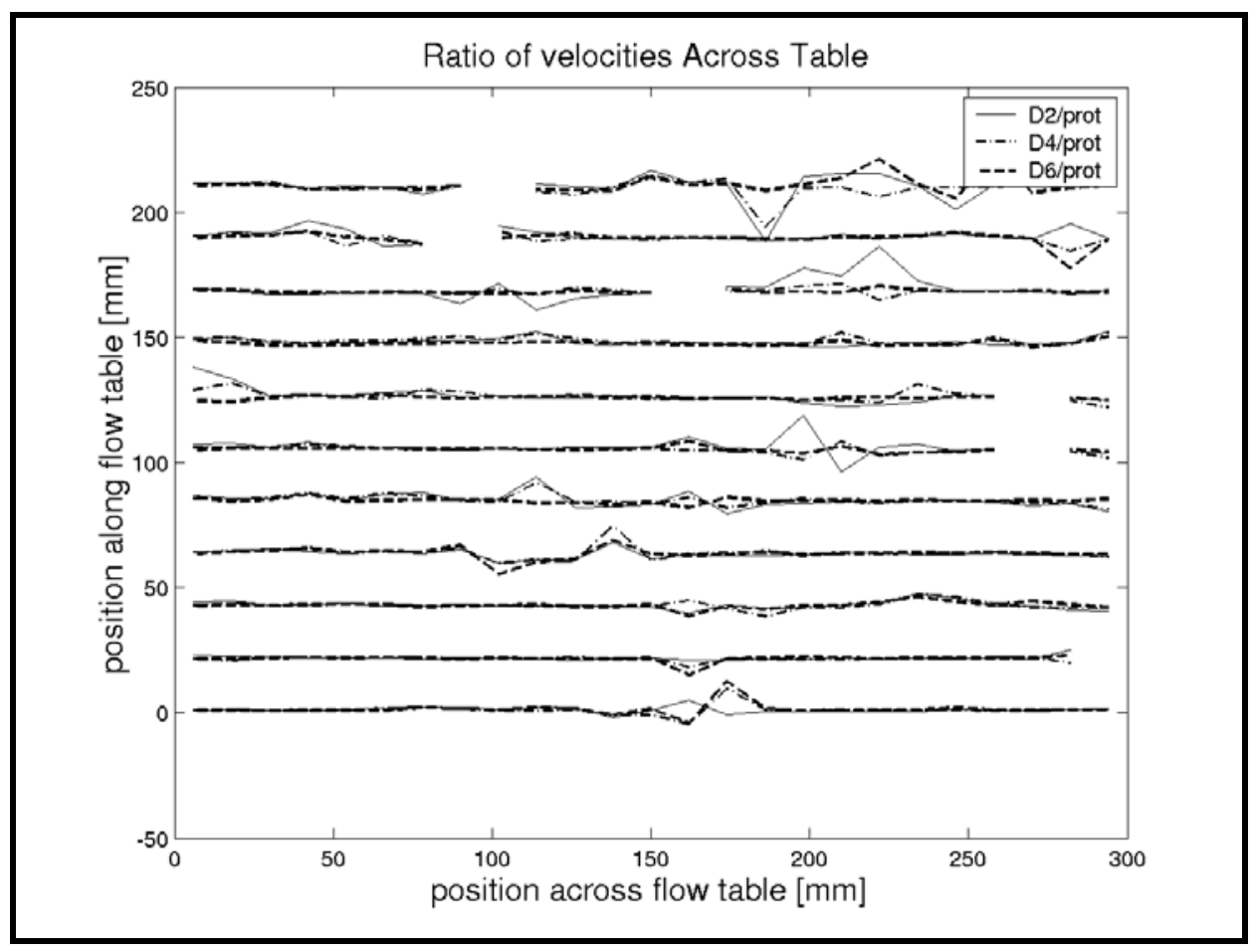

Figure 31. Crossflow velocity ratios, distortion $=2,4,6$ over prototype $\left(N_{Q}=1.0\right.$, Case 2)

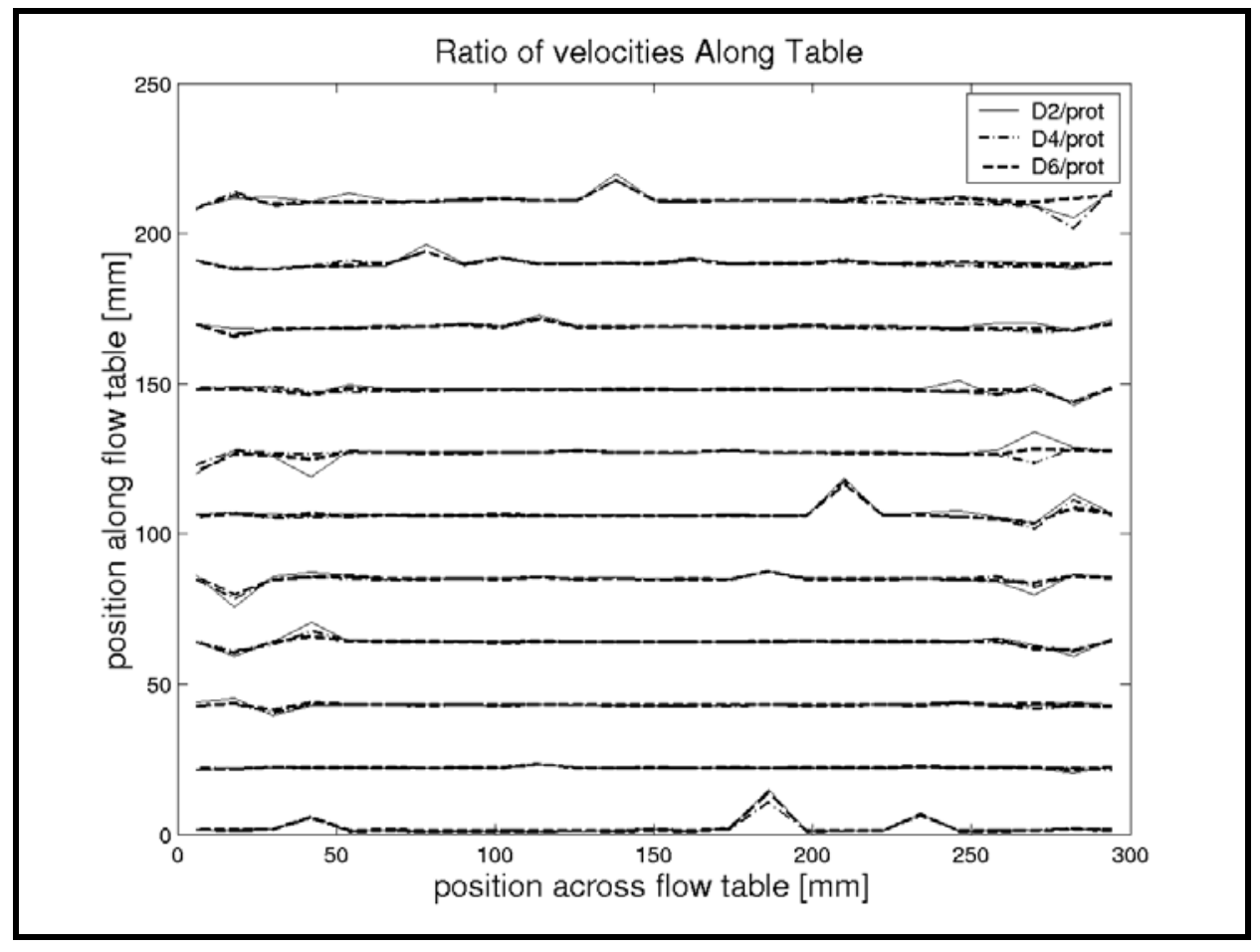

Figure 32. Principal flow direction velocity ratios, distortion $=2,4,6$ over prototype $\left(N_{Q}=1.0\right.$, Case 2$)$ 


\section{Case 3: Flow separation at sloping edge}

The Case 3 tests were similar to Case 1 with the main difference being the angle of the flow separation surface at the gap (see Figure 18(c)). Case 1 used vertical edges as gap boundaries, whereas the Case 3 tests used sloping edges as boundaries. Turbulence generated at sloping boundaries was expected to have significant vertical components; and hence, a turbulent scale effect was expected to occur.

\section{Experiment setup}

The prototype for Case 3 had edge slopes of 1:1, or $45 \mathrm{deg}$. However, different horizontal and vertical length scales resulted in steeper edge slope angles as distortion increased. For model distortions of $\Omega=2,4$, and 6 , the corresponding edge slopes were $63 \mathrm{deg}(2: 1), 75 \mathrm{deg}(4: 1)$, and $80 \mathrm{deg}(6: 1)$, respectively. Figure 33 illustrates the relative distortion of the Case 3 tests and defines the length parameters $X_{p}, X_{m}, Z_{p}$, and $Z_{m}$. The horizontal dimensions across the gap are taken at middepth.

The prototype case, and scaled models of the prototype having distortions ratios $\left(N_{X} / N_{Z}\right)$ equal to 2,4 , and 6 , were tested for two flow rates $(Q=1.5 \mathrm{~L} / \mathrm{sec}$ and $1.0 \mathrm{~L} / \mathrm{sec}$ ). This gave a total of eight experiments. Scaling factors for each test in the series along with relevant values for discharge and key horizontal $\left(X_{p}, X_{m}\right)$ and vertical $\left(Z_{p}, Z_{m}\right)$ dimensions were the same as the first eight tests of Case 1 as listed in Table 5. The horizontal measurement grid was the same as Case 1, and the grid spacing $(\Delta x, \Delta y)$ for each experiment was the same as listed in the first eight rows of Table 6 . Preliminary dye injection into the flow indicated significant vertical structure to the turbulent flow. Consequently, the flow velocity vector field was measured at two depths (one-third and two-thirds of the water depth $(d)$ above the bottom) for each experiment.

\section{Case 3 results}

Partial graphical results obtained from the four tests conducted with a discharge scale of $N_{Q}=1.0(Q=1.5 \mathrm{~L} / \mathrm{sec})$ are presented here as representative of this test configuration. Complete graphical results for all eight Case 3 tests are included in Appendix C.

Measured velocity vectors for the prototype case at an elevation $2 / 3 d$ above the bottom are shown in Figure 34, and the corresponding results from models with distortion 2, 4, and 6 are shown scaled up to prototype in Figures 35, 36, and 37, respectively. Scaling was performed using the scale factors listed in Table 5. A similar distinct jet flow with minimal lateral spreading is seen in all the velocity vector plots recorded at an elevation of $2 / 3 d$ above the bottom.

Corresponding vector plots recorded at a water depth $1 / 3 d$ above the bottom are presented in Figures 38-41. In the prototype test at the lower water depth there is noticeable spreading of the jet as evidenced in Figure 38. Jet spreading decreased as distortion increased and the sloping edge became more vertical as seen in Figures 39-41. 


\section{Distortion with Sloping Edges}
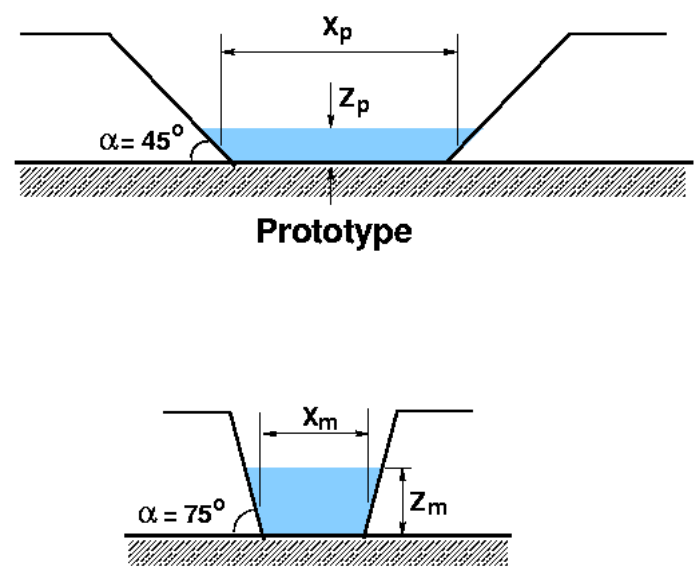

Distortion 4:1

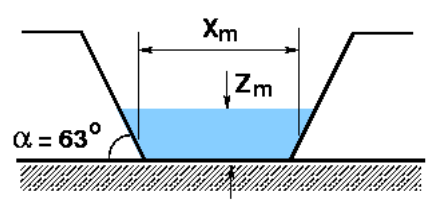

Distortion 2:1

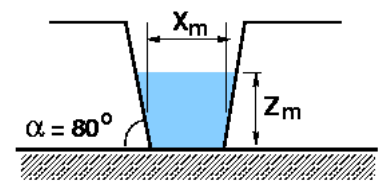

Distortion 6:1

Figure 33. Cross sections showing distortion for sloping edge tests

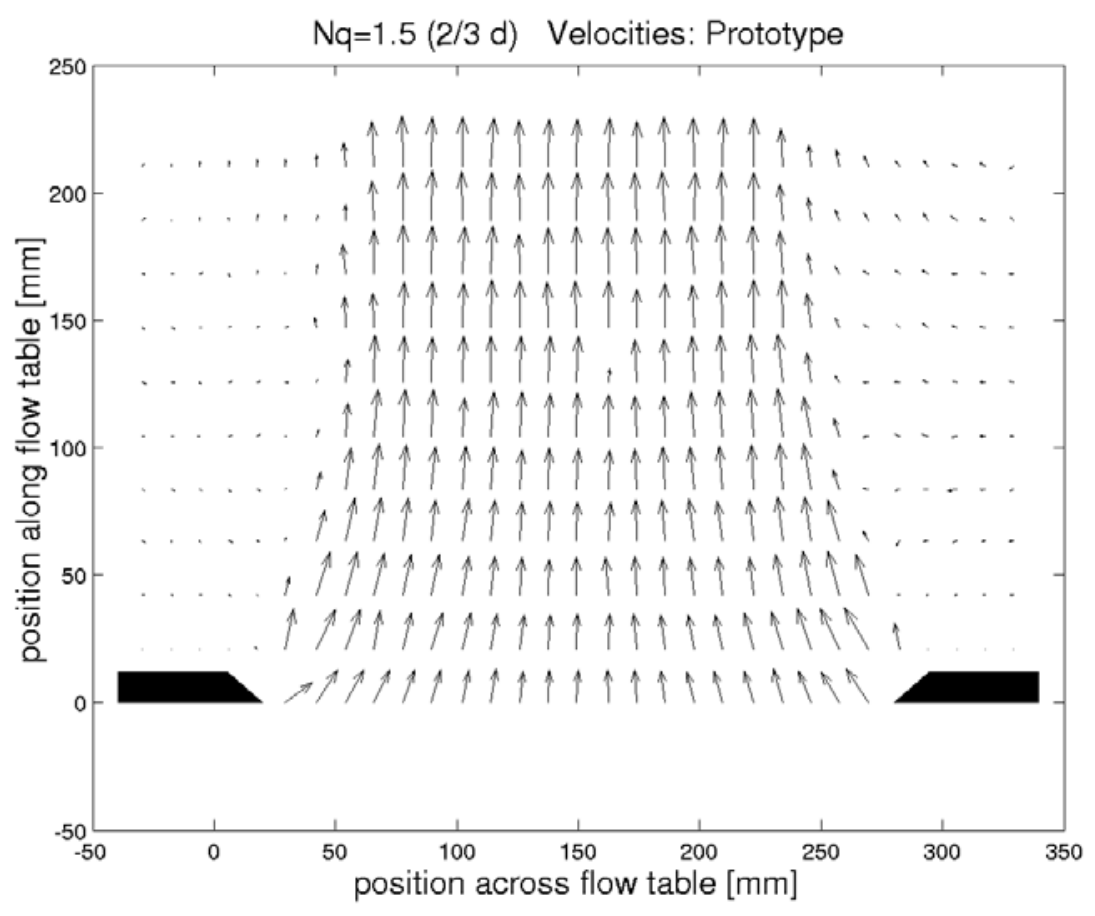

Figure 34. Velocity field at $2 / 3 d, N_{Q}=1.0$, prototype (Case 3) 


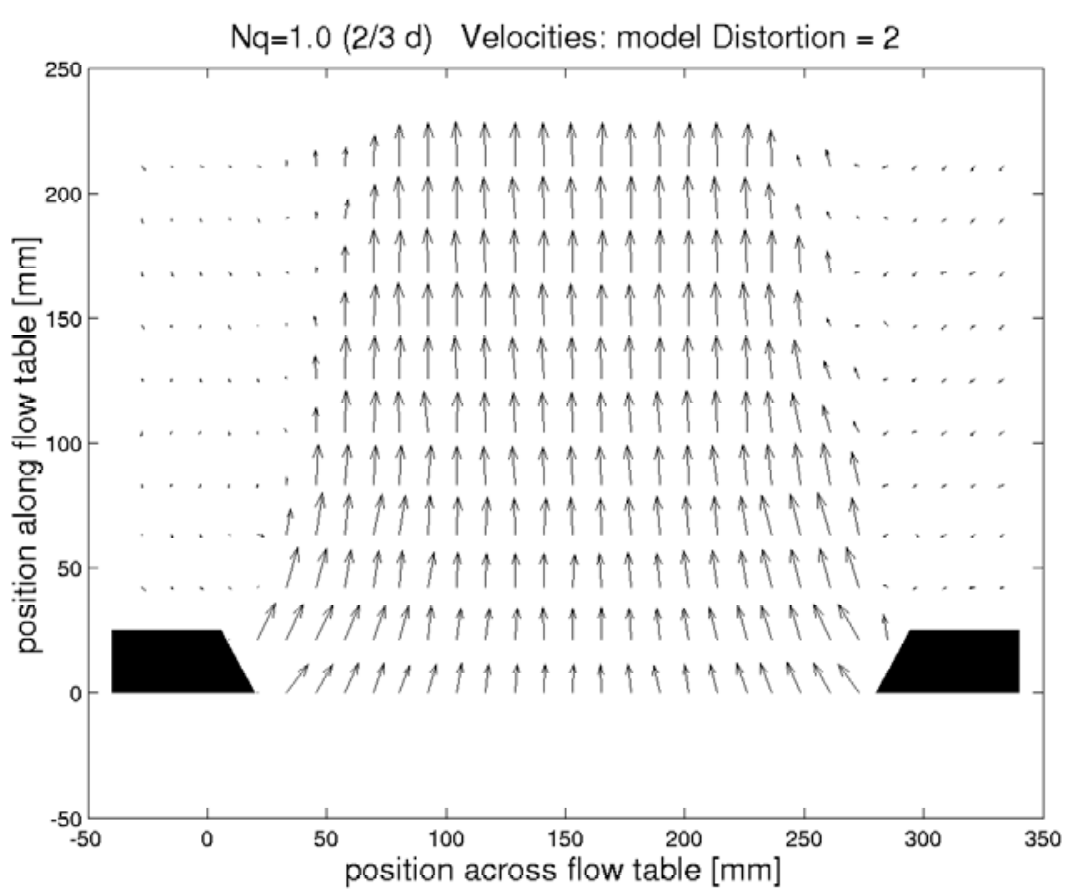

Figure 35. Velocity field at $2 / 3 d, N_{Q}=1.0$, distortion $=2($ Case 3$)$

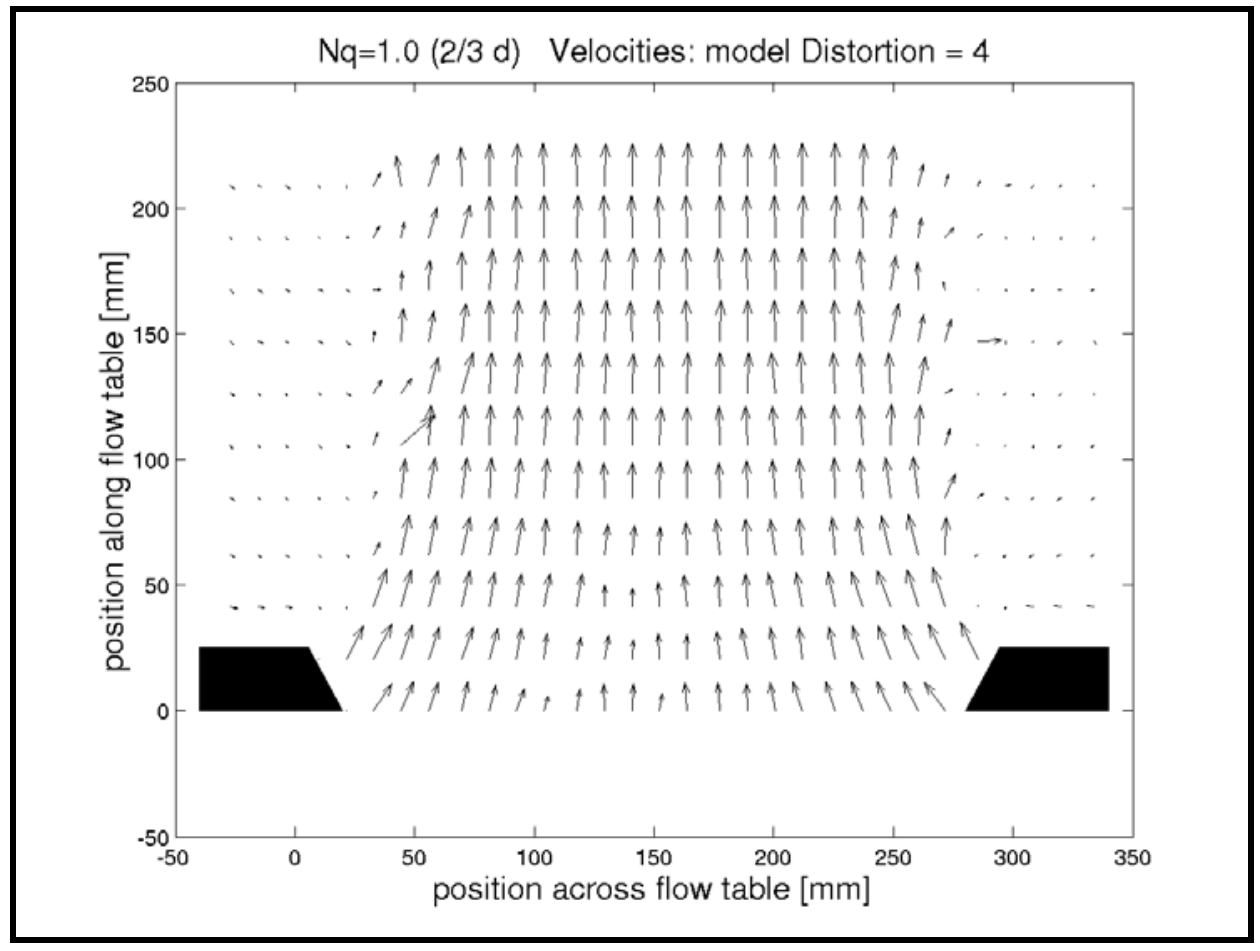

Figure 36. Velocity field at $2 / 3 d, N_{Q}=1.0$, distortion $=4$ (Case 3) 


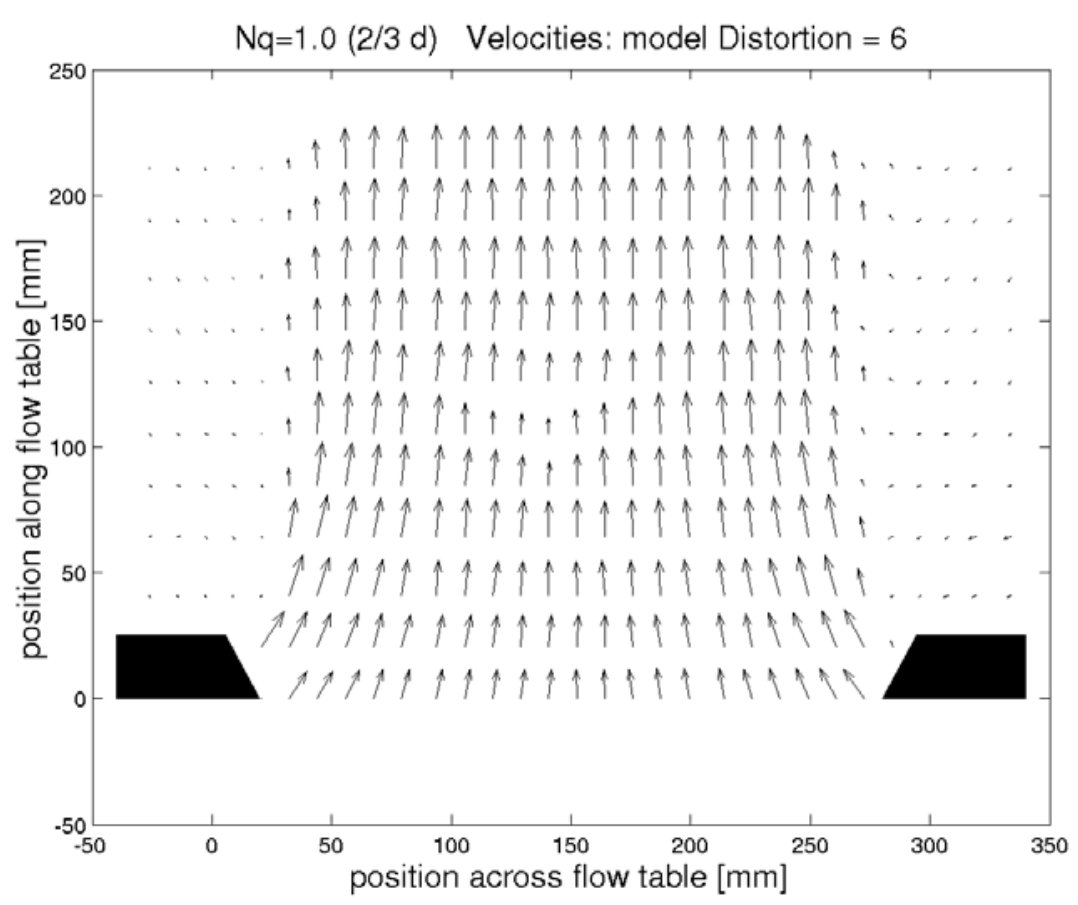

Figure 37. Velocity field at $2 / 3 d, N_{Q}=1.0$, distortion $=6$ (Case 3$)$

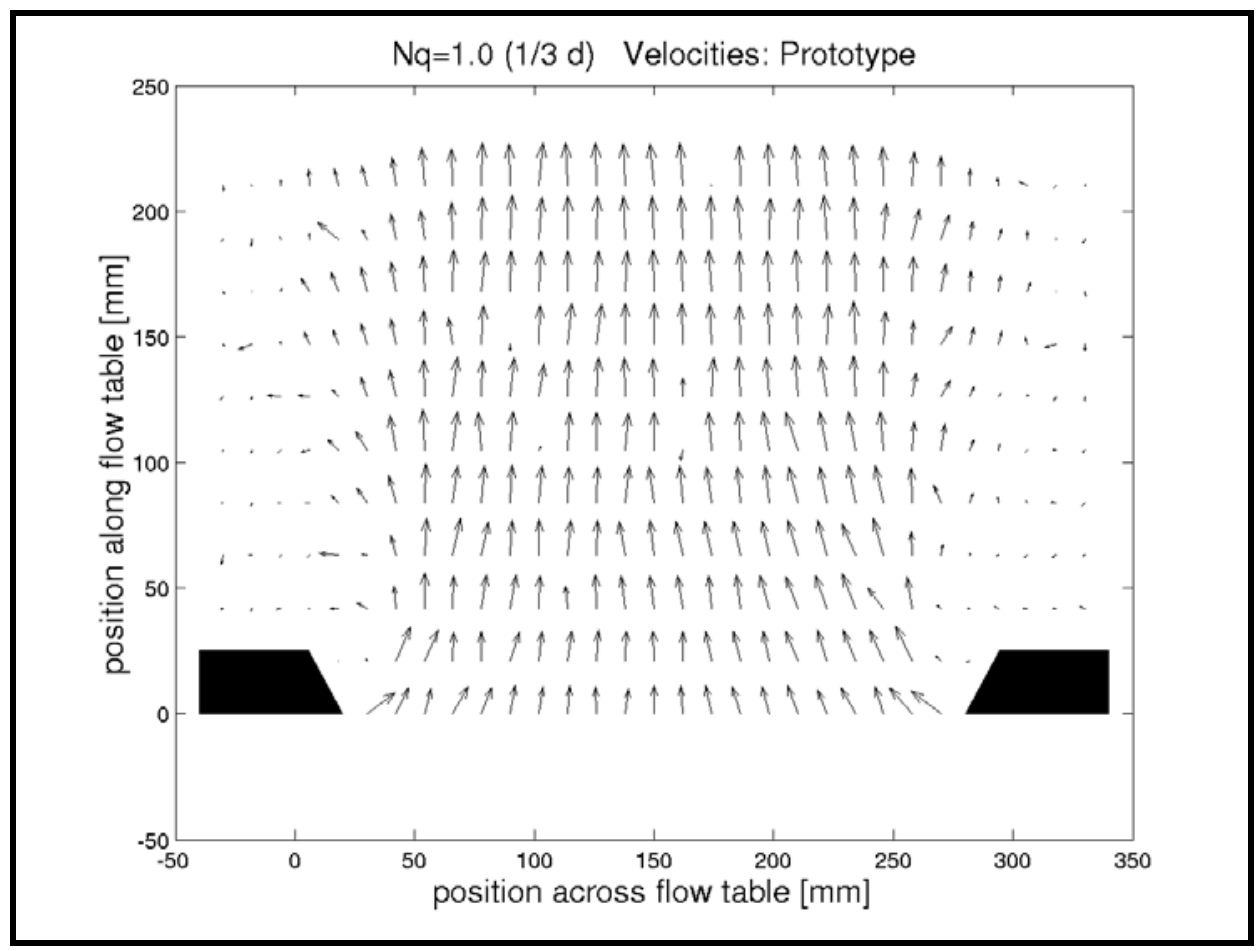

Figure 38. Velocity field at $1 / 3 d, N_{Q}=1.0$, prototype (Case 3) 


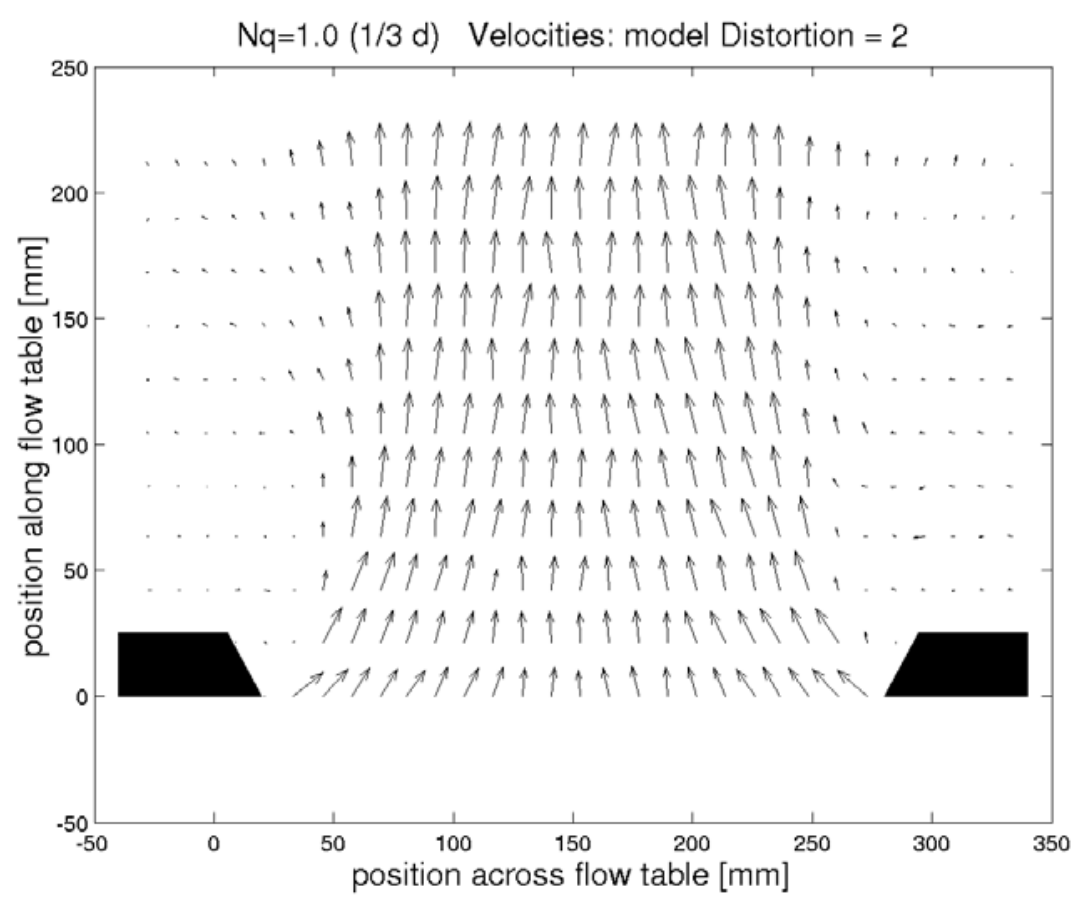

Figure 39. Velocity field at $1 / 3 d, N_{Q}=1.0$, distortion $=2$ (Case 3$)$

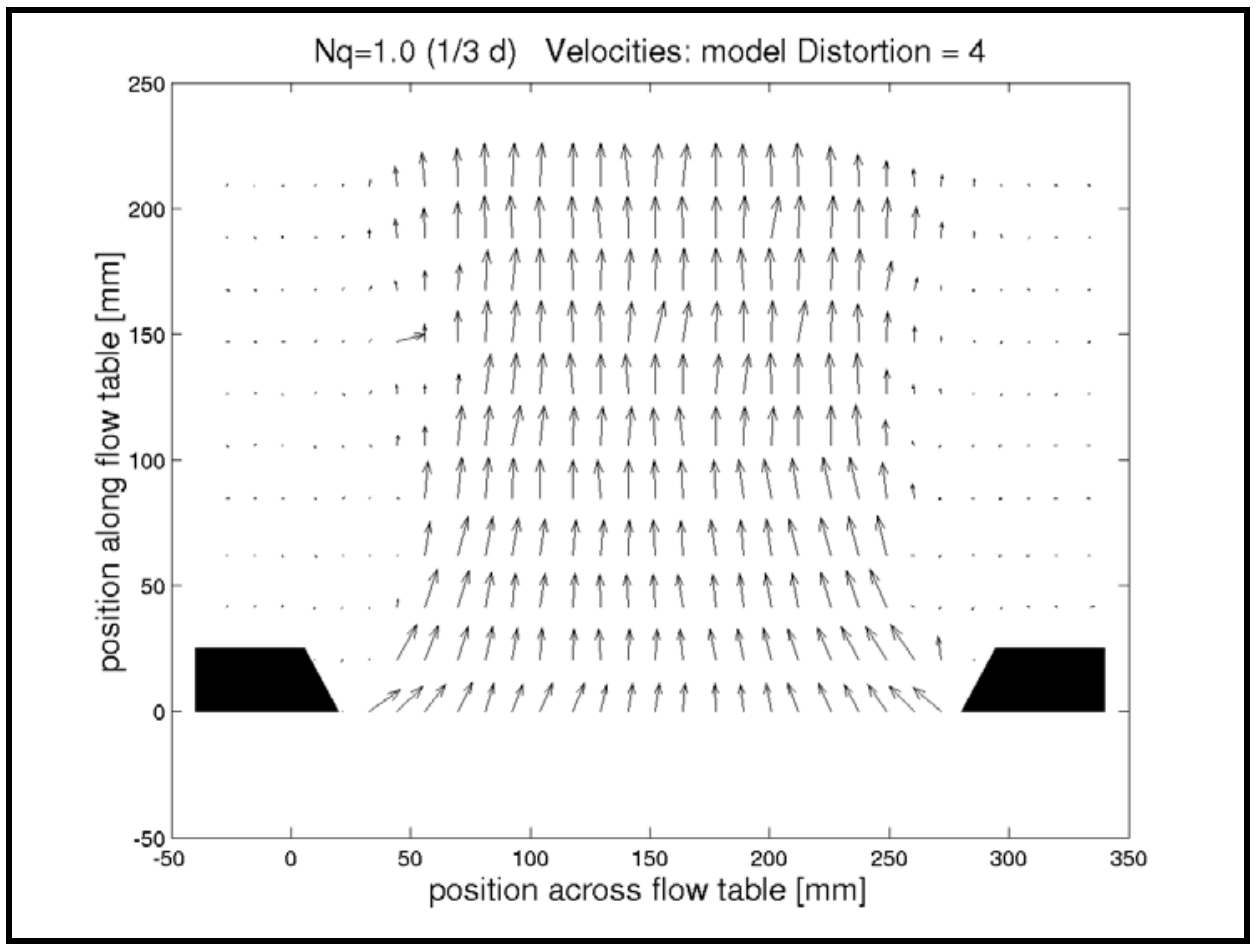

Figure 40. Velocity field at $1 / 3 d, N_{Q}=1.0$, distortion $=4($ Case 3$)$ 


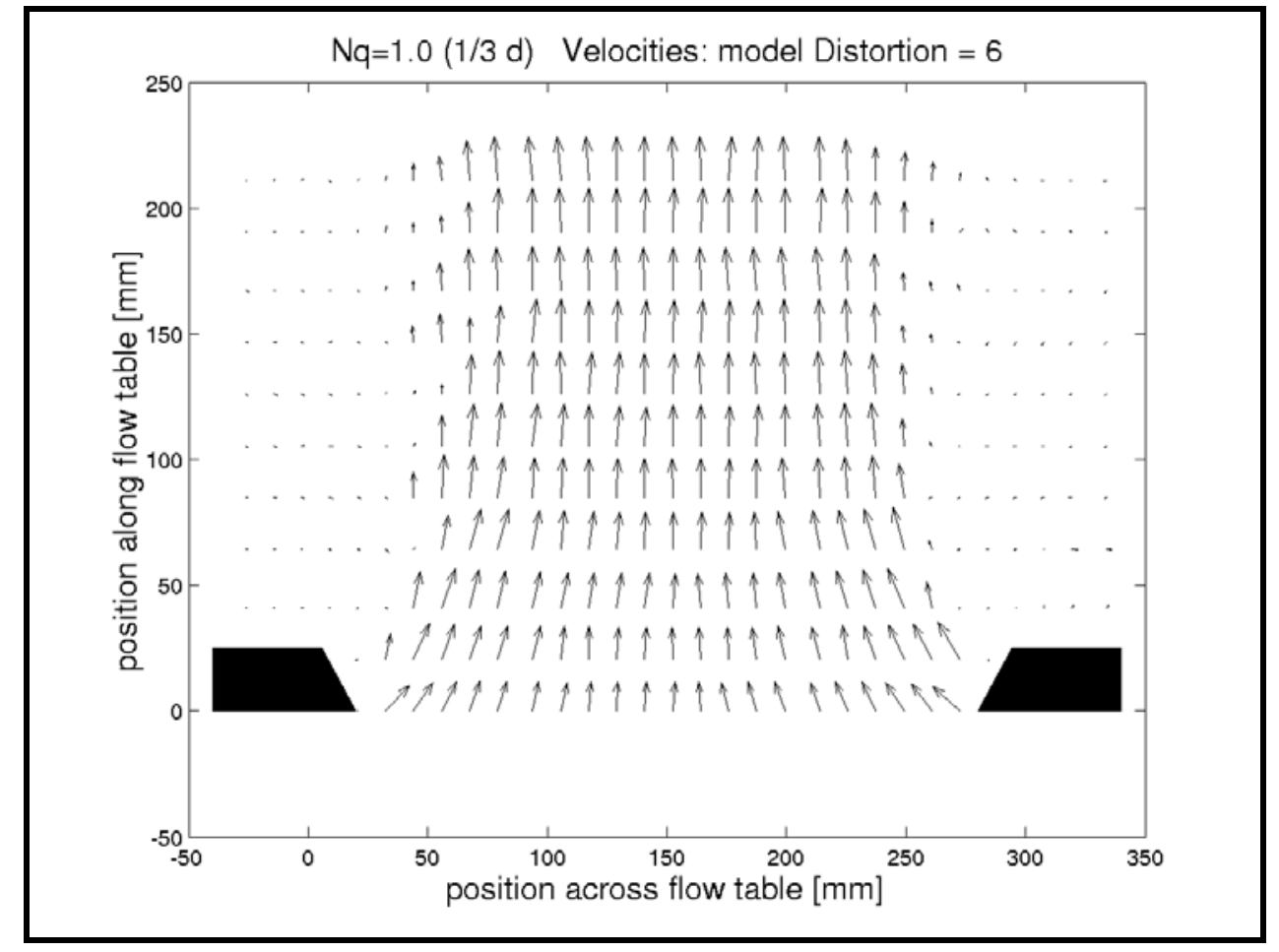

Figure 41. Velocity field at $1 / 3 d, N_{Q}=1.0$, distortion $=6$ (Case 3)

Figures 42 and 43 compare prototype velocity vectors with distortion $=6$ vectors scaled to prototype size at $2 / 3 d$ and $1 / 3 d$ above the bottom, respectively. The distorted model vectors are displaced slightly to the right of the prototype results. For this higher flow rate, there is much better correspondence higher in the water column (Figure 42) while closer to the bottom the prototype jet has spread out more than the distorted model jet (Figure 43). This is a direct consequence of the milder slope of the gap boundaries in the prototype.

The jet created by flow through the sloping-edge gap increases in width toward the free surface. Therefore, between two adjacent horizontal layers there is a strong velocity gradient at the jet boundary in the vertical as well as in the horizontal plane ${ }^{1}$. In addition to fluid being entrained into the jet in the horizontal plane, fluid is also entrained upward at the jet boundary due to the vertical velocity gradient. At, or near, the solid bottom boundary, most of the fluid needed for vertical entrainment must come from the horizontally adjacent quiescent region. Mass conservation then causes the jet to expand horizontally. Higher up in the water column, less fluid is needed from the horizontally adjacent region to fulfill the vertical entrainment requirement; and consequently, the jet does not expand as much in the horizontal plane. In the geometrically distorted model this effect was lessened as the boundary edge became more vertical and the jet boundary velocity gradient between vertical layers became weaker. Similar experiments conducted at the slower flow rate showed the same trend,

\footnotetext{
${ }^{1}$ Dye injected into the flow near the jet boundary was observed moving in a spiral-like motion along the jet boundary in the principal flow direction.
} 


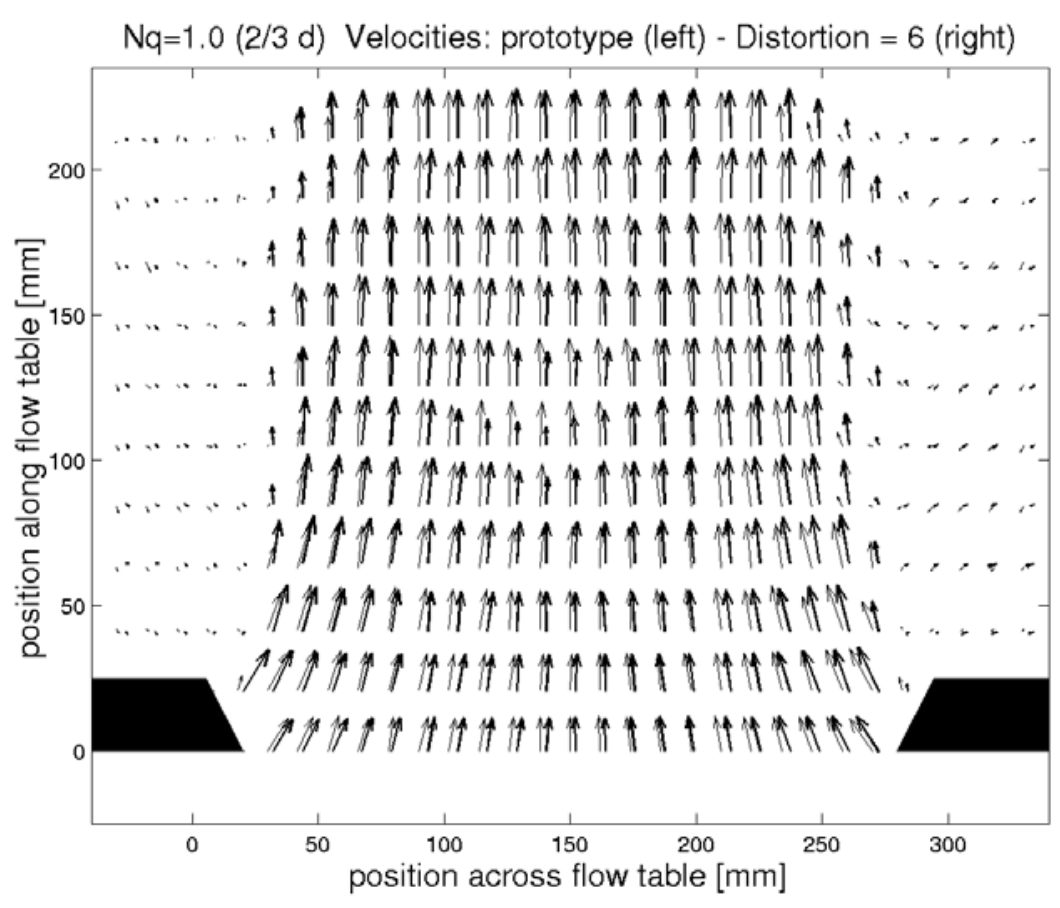

Figure 42. Velocity field at $2 / 3 d, N_{Q}=1.0$, prototype vs. distortion $=6$ (Case 3 )

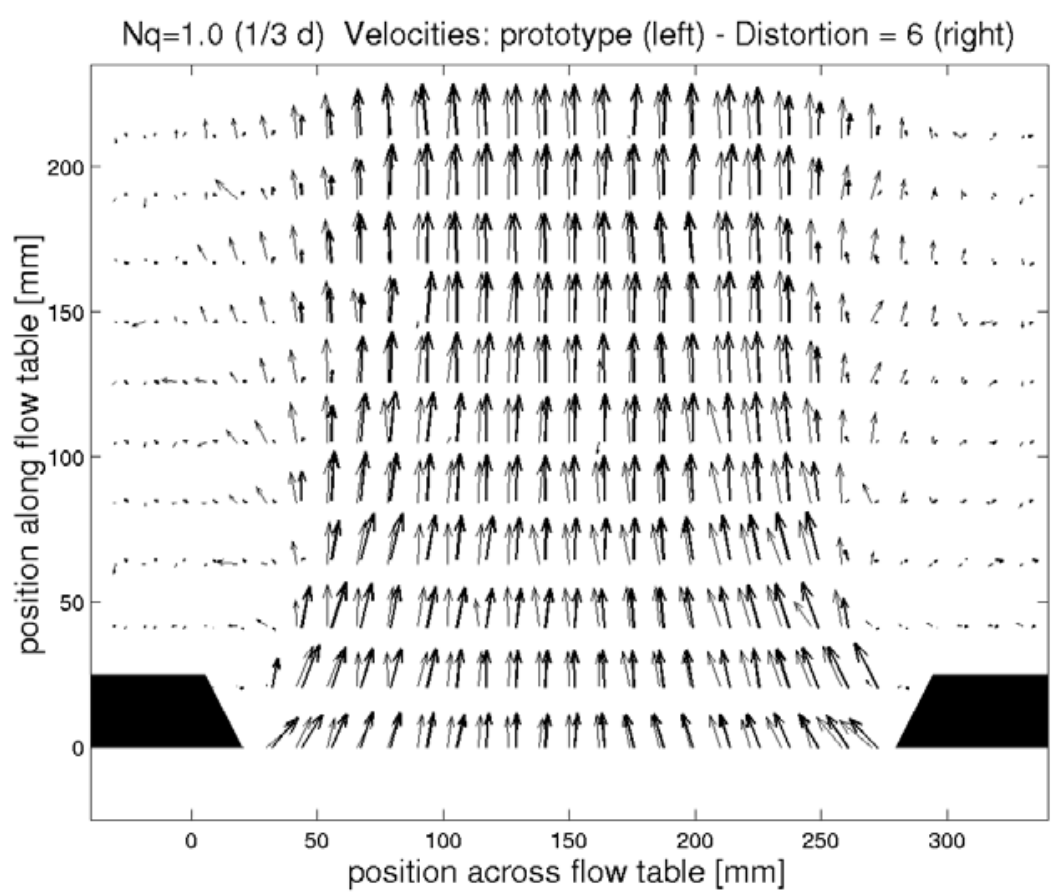

Figure 43. Velocity field at $1 / 3 d, N_{Q}=1.0$, prototype vs. distortion $=6$ (Case 3$)$ 
but not as strongly as exhibited by the higher flow rate (see plots in Appendix C). In fact, dye injections at the slower flow rate suggested that the jet spreading was occurring higher in the water column.

As in the previous tests, the velocity component ratios between prototype and distorted models were determined at each measured point in the flow. Ratios of the crossflow velocity components for experiments with discharge scale $N_{Q}=1.0$ are presented in Figure 44 for elevation $2 / 3 d$ above the bottom, and the corresponding ratios in the principal flow direction are plotted in Figure 45. Ratio plots for lower in the water column at elevation $1 / 3 d$ are shown in Figures 46 and 47. Complete results are included in Appendix C.

\section{Case 3 discussion and conclusions}

Jet flow separation initiated by the sloping edges of gap in the Case 3 experiments was expected to generate turbulence having strong components in both horizontal and vertical planes. This assured that several of the turbulencerelated terms in the equations of motion would not be in similitude in geometrically distorted models.

At the higher flow rate the prototype jet exhibited spreading at lower depths brought about by upward fluid entrainment by the jet. This effect was not as pronounced nearer the free surface. In the distorted scale models, flow separation was caused by much steeper gap edges, and this resulted in less upward fluid entrainment and less spreading of the jet at lower depths. Dye injection near the jet boundary gave visual confirmation of turbulent spiral-like flow structures moving in the principal flow direction. The size of the spirals decreased as model distortion was increased.

The observed scale effect did not have much impact, if any, on the main nonturbulent region of the flow; and away from the jet boundary, the flow entrainment seemed to be in reasonable similitude. The main impact of the scale effect was in the immediate vicinity of the jet boundary, and probably farther downstream where the jet becomes fully turbulent. The fully-turbulent jet in a distorted model will probably not be quite as spread out as the prototype at deeper depths. However, near the free surface, the jet seems to maintain similar velocities and geometries. The severity of this scale effect in a geometrically distorted model, and its impact on study results, must be evaluated according to the specific physical model configuration; but overall, the primary flow structures do not seem to be affected at any great extent. In other words, some of the turbulent structure geometry will be incorrect, but the distorted model should reproduce somewhat similar dominant flow patterns associated with flow separation with velocity magnitudes nearly correct. 


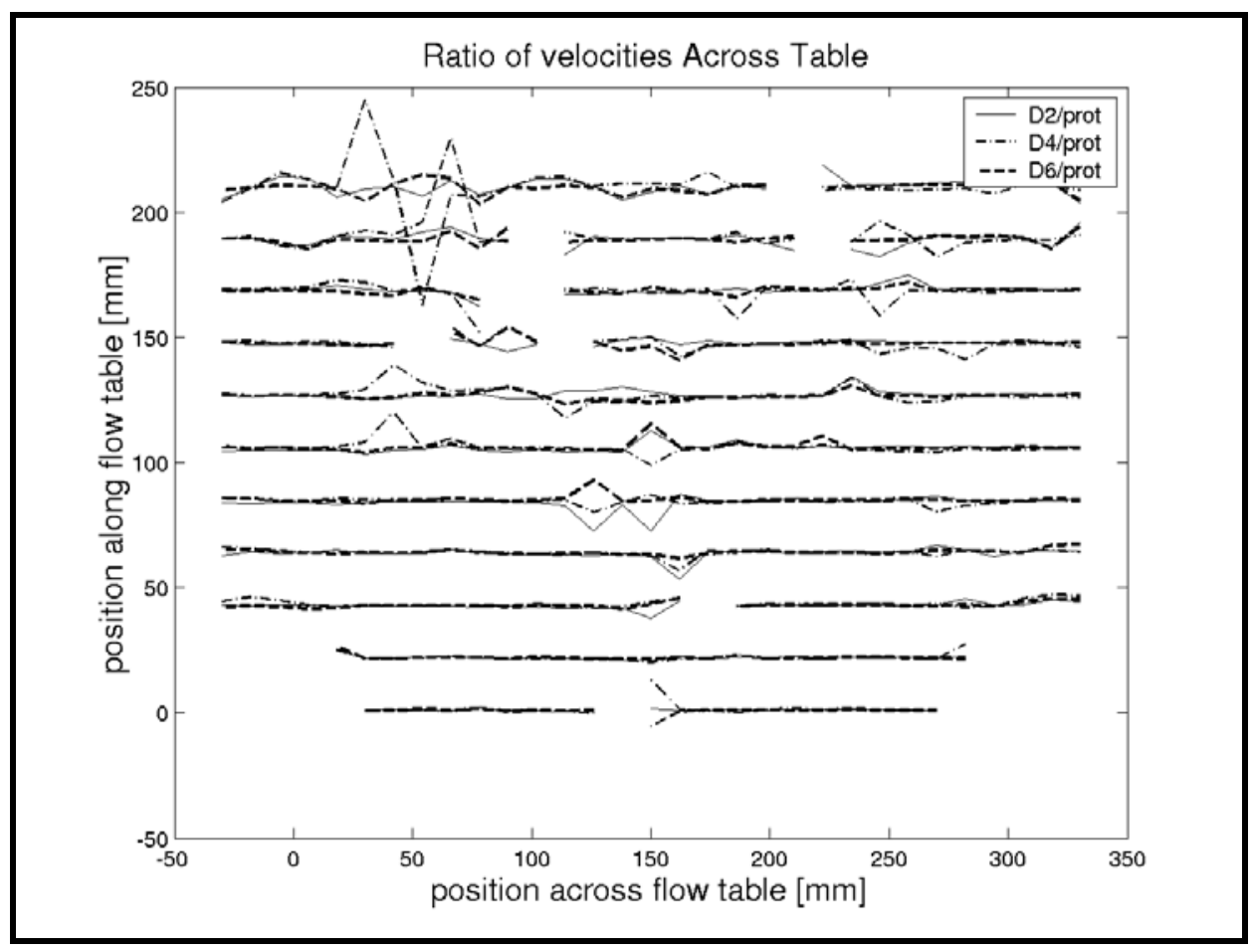

Figure 44. Crossflow velocity ratios at $2 / 3 d$, distortion $=2,4,6$ over prototype $\left(N_{Q}=1.0\right.$, Case 3)

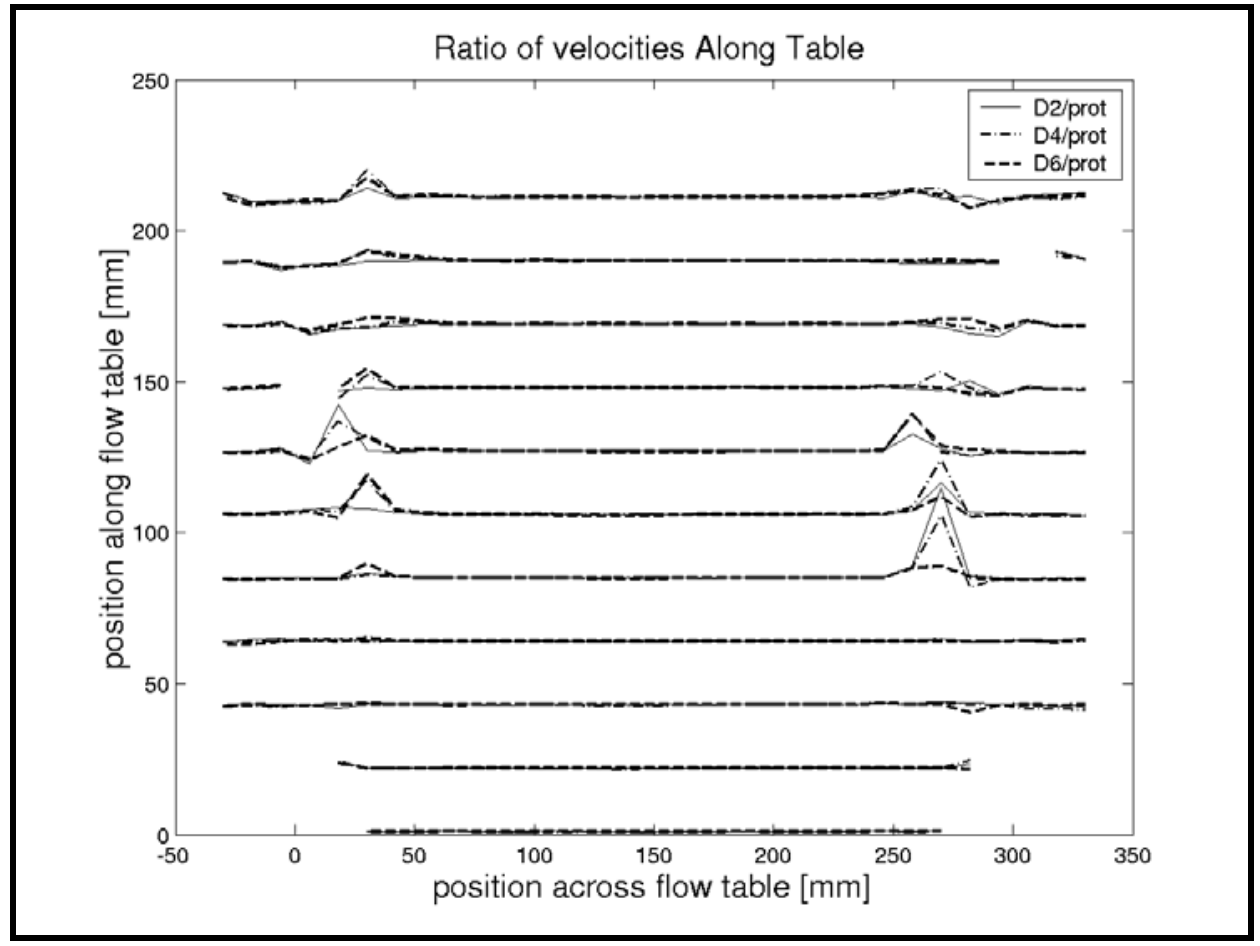

Figure 45. Principal flow direction velocity ratios at $2 / 3 d$, distortion $=2,4,6$ over prototype $\left(N_{Q}=1.0\right.$, Case 3$)$ 


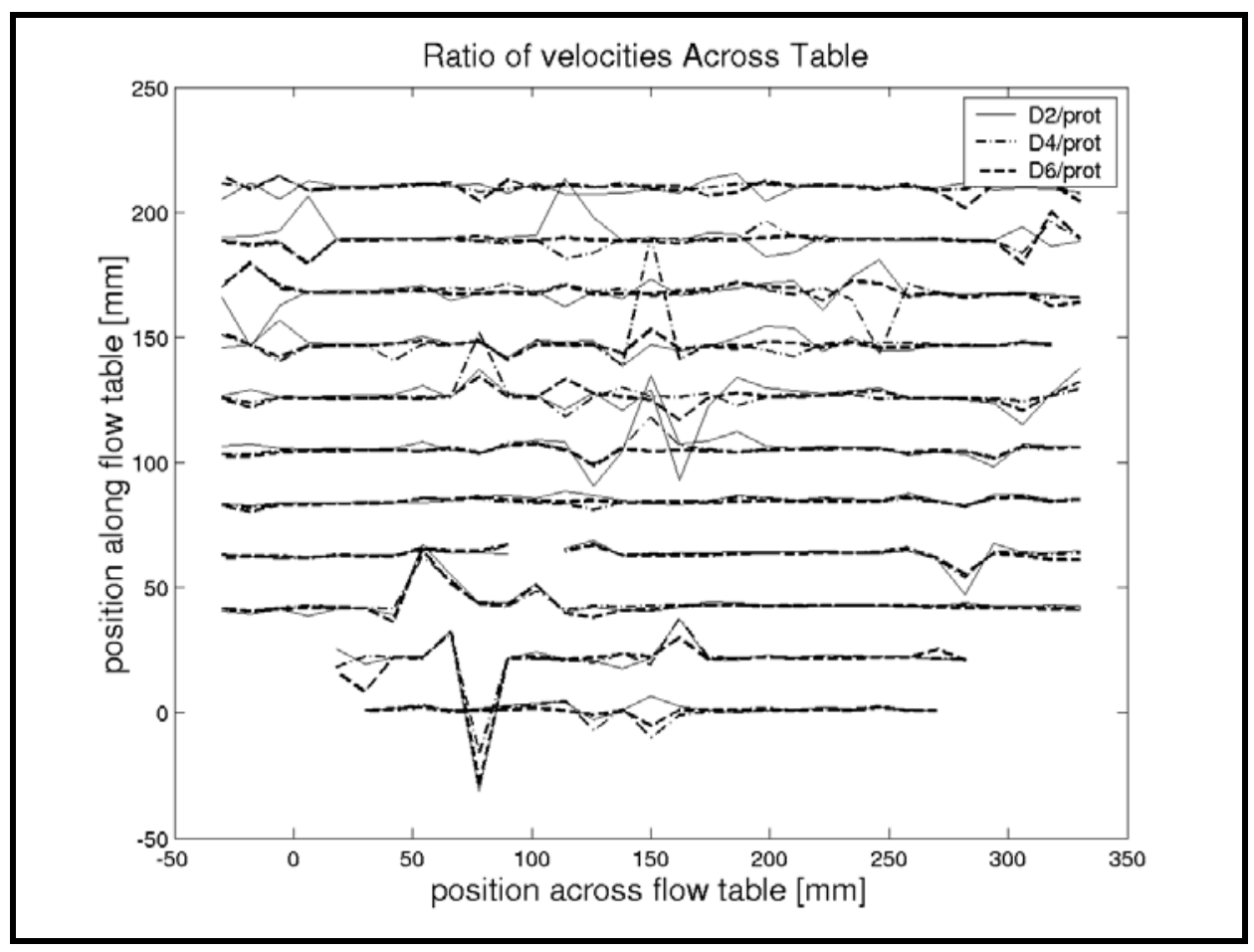

Figure 46. Crossflow velocity ratios at $1 / 3 d$, distortion $=2,4,6$ over prototype $\left(N_{Q}\right.$ $=1.0$, Case 3 )

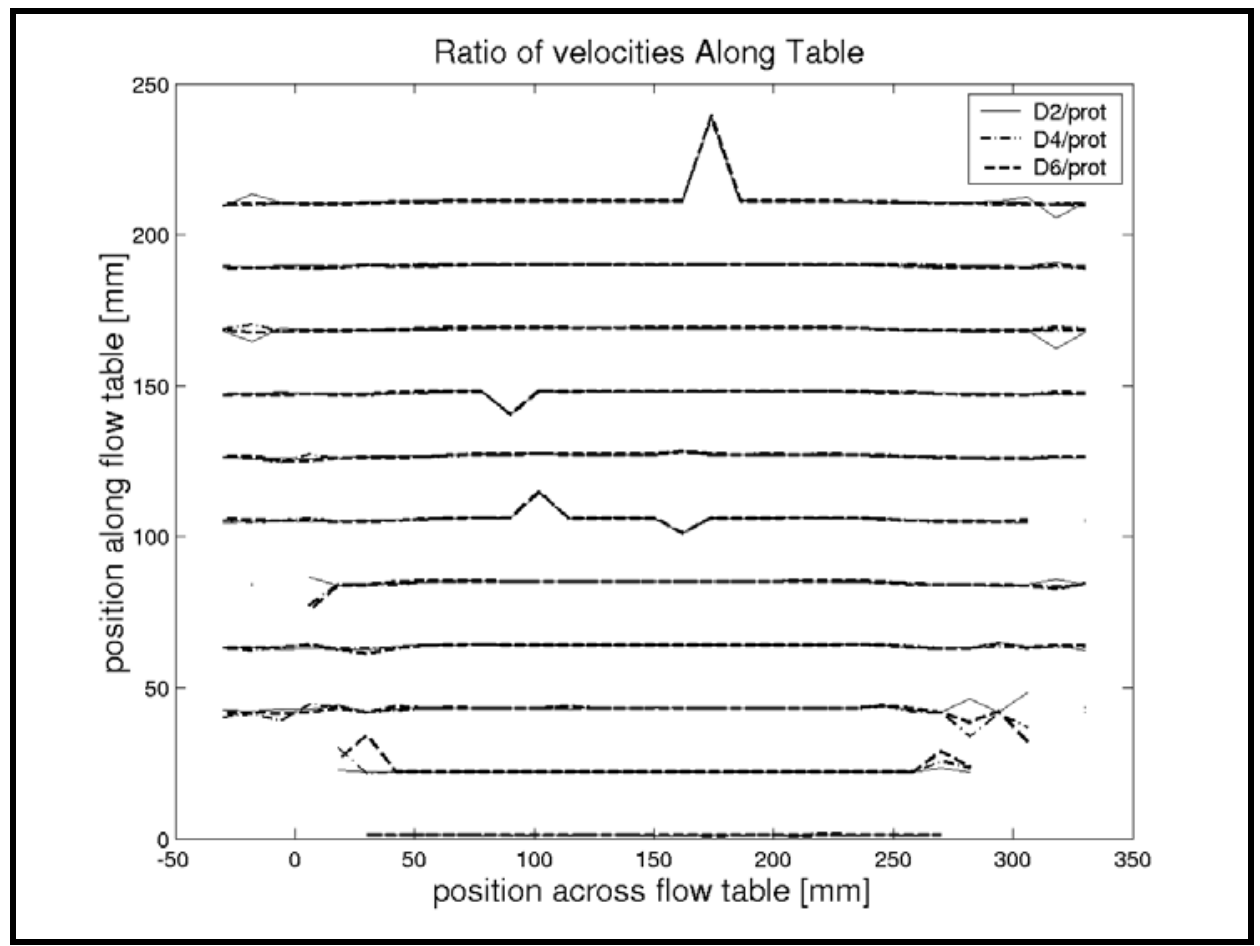

Figure 47. Principal flow direction velocity ratios at $1 / 3 d$, distortion $=2,4,6$ over prototype $\left(N_{Q}=1.0\right.$, Case 3$)$ 


\section{Case 4: Flow Separation at Vertical Step}

The Case 4 tests examined turbulence scaling effects related to flow separation occurring at a vertical step as illustrated in Figure 18(d). Turbulence in the lee of the vertical step was generated by flow separation as uniform flow passed over the vertical step. The resulting turbulence was composed of mostly vertical fluctuations. Because of the relative insignificance of the horizontal turbulent components, only minor scale effects were anticipated.

\section{Experiment setup}

The Case 4 setup consisted of a block placed on the bottom of the flow table with its upstream edge aligned with the gap as illustrated in Figure 48. The gap was formed by vertical edges which generate little vertical turbulence. Velocities were measured in the lee of the downstream edge of the block along the flow center line.

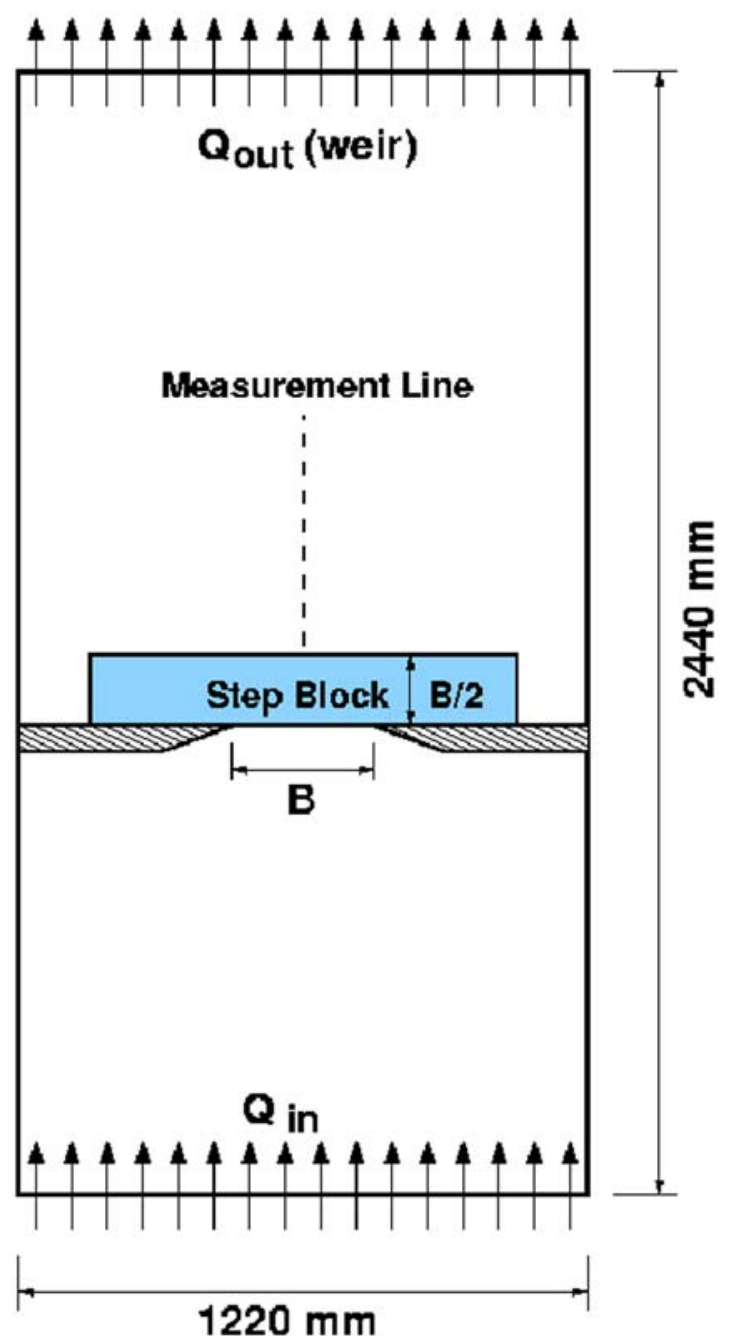

Figure 48. Experiment setup for Case 4 flow over a vertical step 
Table 7 lists the scale factors used in the Case 4 tests, along with values of discharge and key horizontal $\left(X_{p}, X_{m}\right)$ and vertical $\left(Z_{p}, Z_{m}\right)$ dimensions. The $X$ and $Z$ dimensions refer to the flow gap and water depth, respectively. Five horizontal measurement transects were collected at different depths for each experiment. Measurement depths and horizontal line spacings are listed in Table 8. Only the flow rate of $Q=1.5 \mathrm{~L} / \mathrm{sec}$ was tested.

\section{Table 7}

Distortion in Vertical Step Experiment

\begin{tabular}{||l|l|l|l|l|l|l|l|l|l|l||}
\hline \hline Tests & $N_{X} / N_{Z}$ & $N_{Q}$ & $N_{X}$ & $N_{Z}$ & $\begin{array}{l}Q_{p} \\
(\mathrm{~L} / \mathrm{sec})\end{array}$ & $\begin{array}{l}Q_{m} \\
(\mathrm{~L} / \mathrm{sec})\end{array}$ & $\begin{array}{l}X_{p} \\
(\mathrm{~mm})\end{array}$ & $\begin{array}{l}X_{m} \\
(\mathrm{~mm})\end{array}$ & $\begin{array}{l}Z_{p} \\
(\mathrm{~mm})\end{array}$ & $\begin{array}{l}Z_{m} \\
(\mathrm{~mm})\end{array}$ \\
\hline \hline 1 & 1 & 1.0 & 1.000 & 1.000 & 1.5 & 1.5 & 300 & 300 & 37 & 37 \\
\hline 2 & 3 & 1.0 & 1.933 & 0.644 & 1.5 & 1.5 & 300 & 155 & 37 & 58 \\
\hline 3 & 6 & 1.0 & 2.930 & 0.488 & 1.5 & 1.5 & 300 & 102 & 37 & 77 \\
\hline
\end{tabular}

\begin{tabular}{||l|l|l|l||}
\hline \multicolumn{4}{|l|}{$\begin{array}{l}\text { Table } 8 \\
\text { Measurement Geometry of Case } 4 \text { Vertical Step Experiment }\end{array}$} \\
\hline \hline Tests & $N_{X} / N_{Z}$ & $\begin{array}{l}\Delta y \\
(\mathrm{~mm})\end{array}$ & $\begin{array}{l}\text { Measurement Depth } \\
(\mathrm{mm} \text { above bottom })\end{array}$ \\
\hline 1 & 1 & 5 & $3,7,12,18,27,35$ \\
\hline 2 & 3 & 2.6 & $5,11,18,30,43$ \\
\hline 3 & 6 & 1.7 & $6,14,25,38,54$ \\
\hline
\end{tabular}

\section{Case 4 results}

Comparison between the prototype and models with distortion equal to 3 and 6 are displayed in Figure 49. The figure presents a cross section along the flow center line with flow moving from left to right. The vertical step is drawn on the lower left of the figure, and the plot dimensions are those of the prototype. The magnitudes of horizontal velocities measured along the transect lines at each depth are represented by a vertical distance. Prototype magnitudes are given as vertical lines, whereas the distorted model magnitudes (scaled up to prototype) are shown as lines connecting points. No comparisons were available for the prototype transect closest to the free surface.

In the nonturbulent flow region above the vertical step, comparisons are judged to be very good. Note, however, at elevation of $27 \mathrm{~mm}$ (1.06 in.)the prototype horizontal velocity magnitudes showed a gradual decrease with increasing distance downstream. This decrease was not evident in the distorted models. Because similar prototype decrease did not occur at other depths, it was concluded that the prototype measurements at the $27-\mathrm{mm}$ (1.06-in.) elevation probably contained some time-dependent measurement error such as drift of the voltage offset level. 


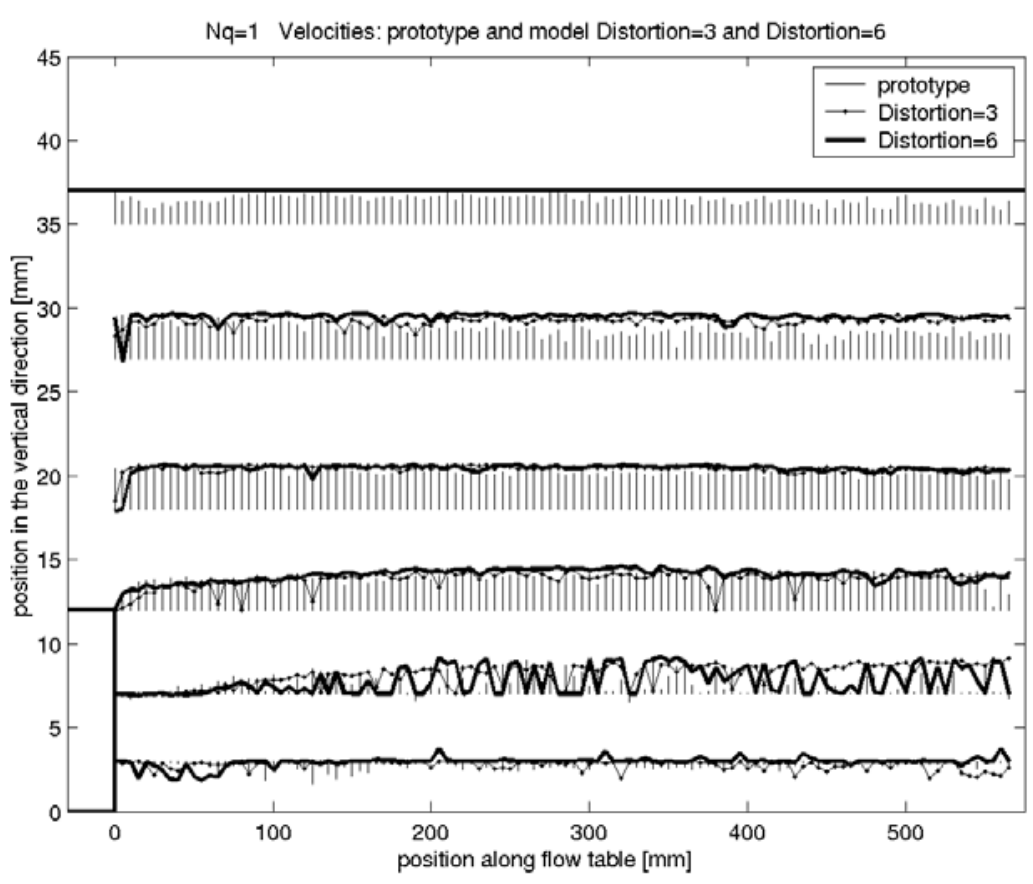

Figure 49. Comparison of horizontal flow magnitudes in lee of a vertical step

Horizontal velocities at the elevation of the step show remarkable correspondence considering this is near the flow separation boundary. Farther down in the entrainment region, the plots show similar magnitude envelopes, but the details differ due to the turbulent nature of the flow. Nevertheless, the comparison was judged to be reasonable. Close to the bottom, the flow is almost motionless.

\section{Case 4 discussion and conclusions}

The vertical step generated flow separation turbulence predominantly in the vertical plane with only weak horizontal turbulent fluctuations. Consequently, the turbulence terms in the equations of motion that are not in similitude (i.e., terms containing products of the horizontal and vertical fluctuations) are small relative to the dominant terms. Therefore, turbulent scale effects arising from the distorted models were very minor, and good similitude was seen throughout the flow field. Details within the entrainment region exhibited variations in the average due to the turbulence, but the bounds of the magnitudes were similar. The flow separation boundary seemed to be well reproduced.

\section{Conclusions from Turbulence Scale Effects Experiments}

An extensive set of experiments was conducted to determine potential turbulence scale effects that might arise due to flow separation in geometrically distorted physical models. Four distinct cases were examined: (a) flow 
separation at a vertical edge resulting in a free jet, (b) flow separation at a vertical edge with a constrained jet, (c) flow separation at a sloping edge resulting in a free jet, and (d) flow separation at a vertical step. In all cases the impact of model distortion was evaluated by comparing the measured velocity fields of the prototype experiment with the velocity fields of the distorted models scaled to prototype size using appropriate scale ratios. Judging whether or not good similitude existed between model and prototype was subjective, and good correspondence was noted where variations between vector fields were thought to be small enough to have been caused by measurement error or small misalignment of boundary geometry between experiments. The conclusions from the turbulence scale effects experiments are as follows:

a. Flow separation and turbulence generated at vertical edges will not have a scale effect in geometrically distorted models because vertical turbulent fluctuations are small.

$b$. Flow separation at vertical edges with constraining downstream jetties will be similitude in distorted models with good correspondence in resulting flow patterns in the main flow and in the entrainment region.

c. Flow separation at a vertical step where the turbulence is manifested primarily in the vertical plane will not have any significant scale effect in geometrically distorted models.

d. Flow separation initiated by a sloping edge will exhibit a scale effect in geometrically distorted models. However, the scale effect is strongest near the bottom and appears to lessen closer to the free surface. Also, the scale effect seemed to be restricted to the immediate vicinity of the jet boundary; however, potential impacts farther downstream were not evaluated due to the limited measurement region of the experiments.

e. Evaluation of potential turbulent scale effects in proposed distorted physical models requires good understanding of dominant flow patterns in the prototype along with knowledge of boundaries where flow separation and/or turbulence generation might occur. Evaluation must be site-specific.

f. Importance of turbulent scale effects is a geometrically distorted physical model relates directly to the problem being studied, and whether or not flow turbulence is a dominant forcing of that problem. 


\section{Impact of Dredging Planform}

\section{Introduction}

At present, the upstream and downstream ends of the dredged region of the Port of Anchorage have vertical sidewalls cut out of hard, nonerodible material. The dredge transitions are oriented at a $30 \mathrm{deg}$ angle to the shoreline. The abrupt vertical transition may cause quiet flow regions which allow suspended sediment to deposit in the harbor. The objective of this task was to test the hypothesis that sloping dredge planform transitions on both ends of the harbor would decrease areas of flow reduction and increase harbor flushing.

Comparisons were made between existing dredging practice and the proposed dredging planform featuring sloping transitions. Both alternatives were fabricated and placed on the precision flow table for testing. Flow fields near the transition were quantified using the laser Doppler velocimeter, and dye injection was used as a qualitative tool to evaluate the two dredge planforms.

\section{Experiment Setup}

A rectangular flow channel of width $380 \mathrm{~mm}$ (14.9 in.) was created on the flow table using Plexiglas blocks as illustrated in Figure 50. The water depth was set to $44 \mathrm{~mm}(1.7 \mathrm{in}$.), and a flow rate of $1.7 \mathrm{~L} / \mathrm{sec} 3(.5 \mathrm{pints} / \mathrm{sec})$ was used for the experiments. Average flow velocity over the channel cross section was approximately $10 \mathrm{~cm} / \mathrm{sec}(3.9 \mathrm{in} . / \mathrm{sec})$. Relevant scaling factors (prototype-tomodel ratios) for the dredge transition models are listed in Table 9 along with approximate model equivalences. Model geometric distortion was $\Omega=N_{X} / N_{Z}=$ 5 for this simplified model of the Port of Anchorage dredging transitions.

Two dredging transition models were fabricated out of Plexiglas by the ERDC Model Shop. The first model, shown on the left side of Figure 51, featured a vertical transition along the dredge cut that is sloped at $30 \mathrm{deg}$ to the shoreline. This represented the present dredging practice. The second model, shown on the right side of Figure 51, is similar except for the sloping transition from the deeper dredged area up to the existing bottom elevation.

In the first tests each transition was oriented as shown in Figure 50 with the deeper portion upstream of the shallower portion. This represented flow leaving the harbor over the transition. Next, the transitions were reversed on the flow table to represent tidal flow from the shallower area entering the harbor over the transition. Velocities on the horizontal portion adjacent to the transition were 
measured over a grid (see Figure 50) at a water depth equal to about half the maximum transition height (i.e., $10 \mathrm{~mm}$ (0.39 in.)).

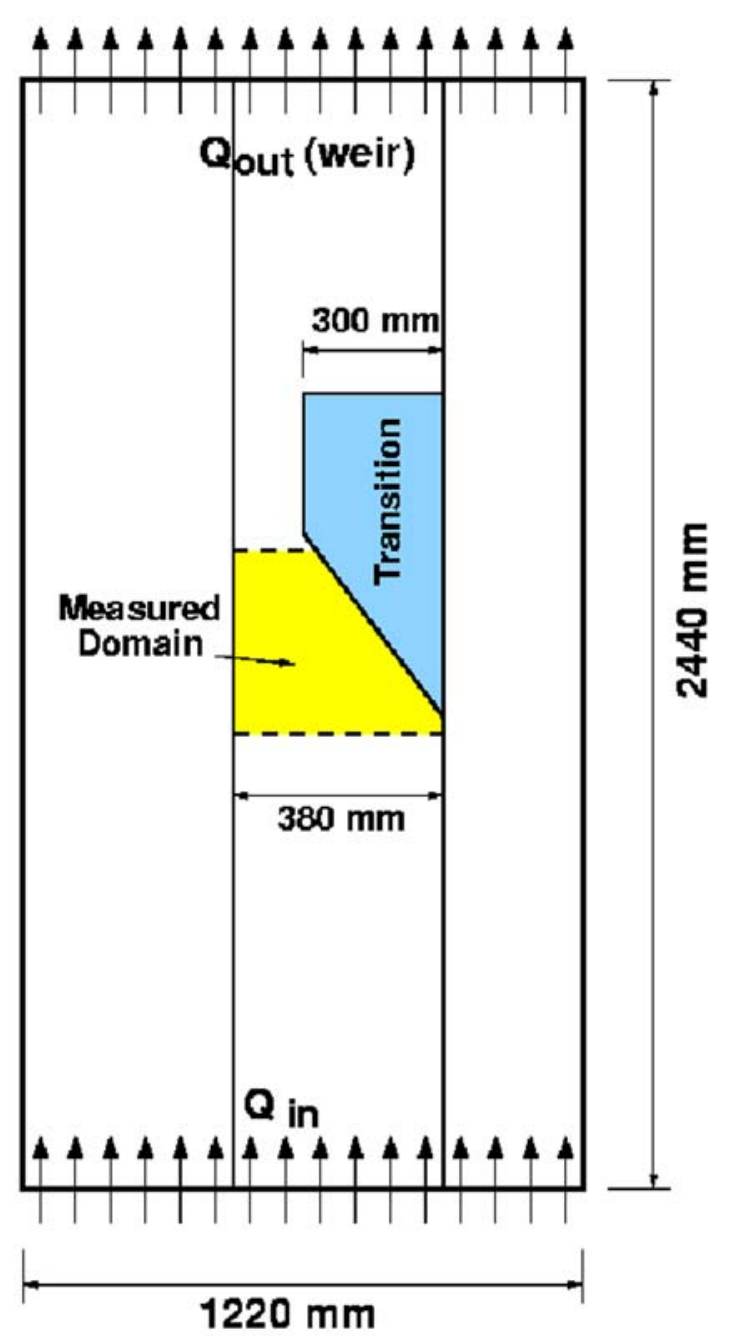

Figure 50. Experiment setup for harbor dredging planform configuration

\begin{tabular}{|c|c|c|}
\hline \multicolumn{3}{|c|}{$\begin{array}{l}\text { Table } 9 \\
\text { Scale Ratios for Dredge Transition Model }\end{array}$} \\
\hline Scale & Scale Value & Model Equivalence \\
\hline Horizontal scale & $N_{X}=1,200$ & $100 \mathrm{ft} \approx 1 \mathrm{in}$. \\
\hline Vertical scale & $N_{Z}=240$ & $20 \mathrm{ft}=1 \mathrm{in}$. \\
\hline Velocity scale & $N_{V}=\sqrt{N_{Z}}=15.5$ & $1.55 \mathrm{~m} / \mathrm{sec}=10 \mathrm{~cm} / \mathrm{sec}$ \\
\hline Discharge scale & $N_{Q}=N_{X} N_{Z}^{3 / 2}=4,461,680$ & $7,585 \mathrm{cu} \mathrm{m} / \mathrm{sec}=1.7 \mathrm{~L} / \mathrm{sec}$ \\
\hline
\end{tabular}




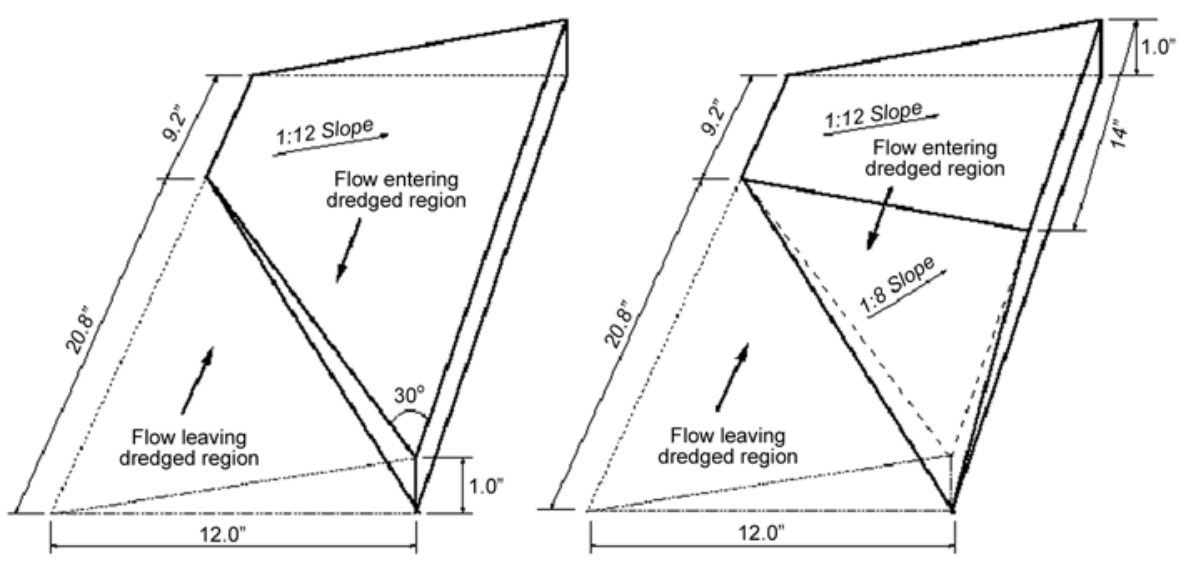

Figure 51. Dredge transition planform configurations

\section{Dredge Transition Test Results}

\section{Flow leaving dredged area}

The first case tested was flow leaving the deeper dredged area and passing over the transition. Measured velocity vectors in the dredged area adjacent to the dredge transition are shown in Figure 52 for the vertical transition and Figure 53 for the sloping transition.

For the vertical dredge transition, the vertical distance was highest at the lower right side of Figure 52, and here the middepth velocity vectors aligned with the transition and increased slightly in magnitude. This vector realignment lessened as the height of the vertical step decreased. However, the effect of the vertical transition was mainly in redirecting the flow; velocity magnitudes were not appreciably decreased, and this lowered the suspended sediment deposition potential.

The flow pattern was only slightly different in the case of a sloping transition as shown by the vectors in Figure 53. The vectors were more uniform at middepth because of a smoother transition to the shallower region, and deposition potential was even less than for the vertical transition.

\section{Flow entering dredged area}

The dredge transition pieces were reversed in the flow table to simulate tidal flow entering the dredged area. Measured velocity vectors in the area to the lee of the dredge transition are shown in Figure 54 for the vertical transition and Figure 55 for the sloping transition.

As water flows over the vertical transition, flow separation occurs at the step which creates a region of turbulence in the immediate lee of the step. This is weakly illustrated by Figure 54, where flow is from the bottom to top in the figure. Dye injection upstream of the vertical transition revealed a helical flow 


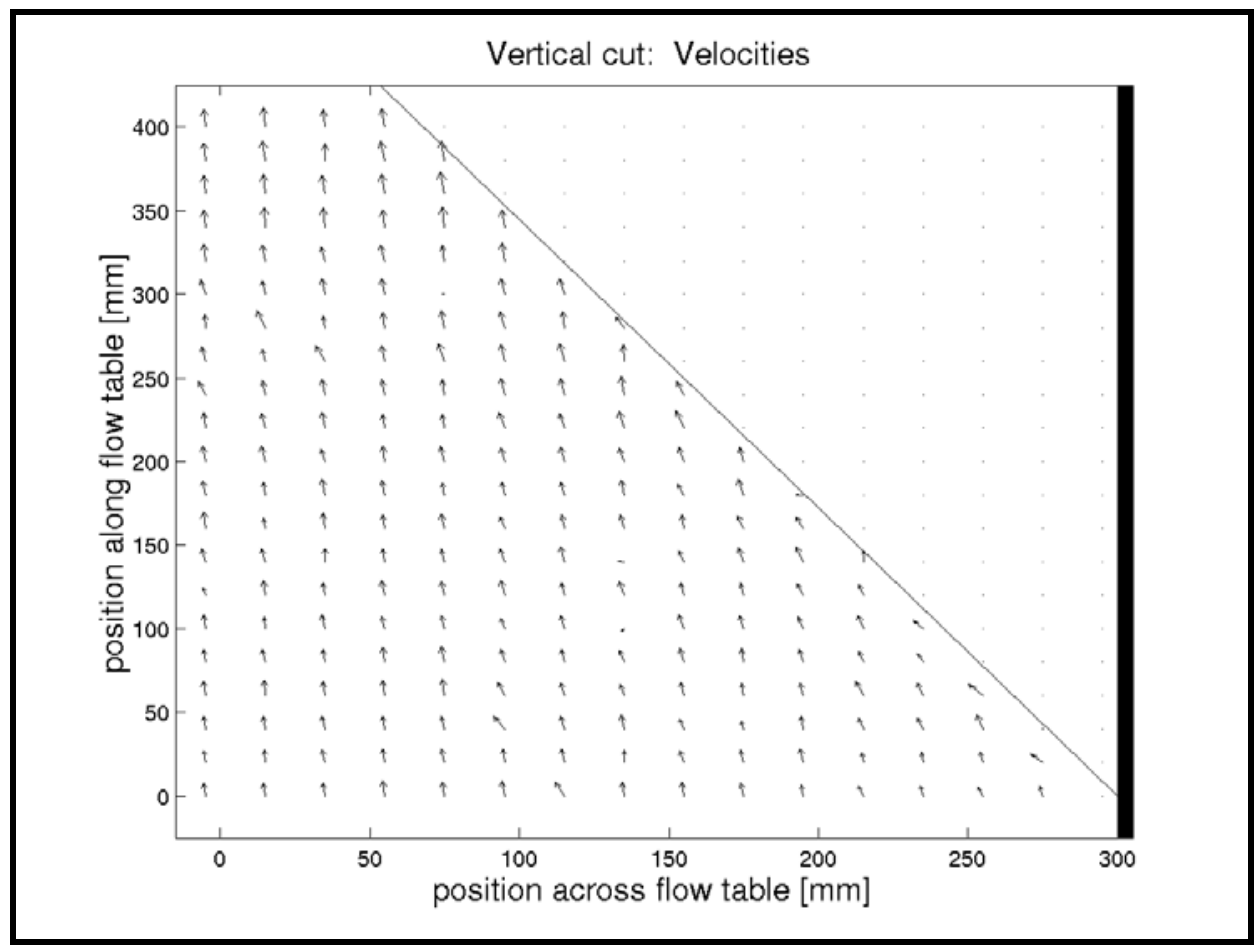

Figure 52. Flow leaving dredged region over vertical transition

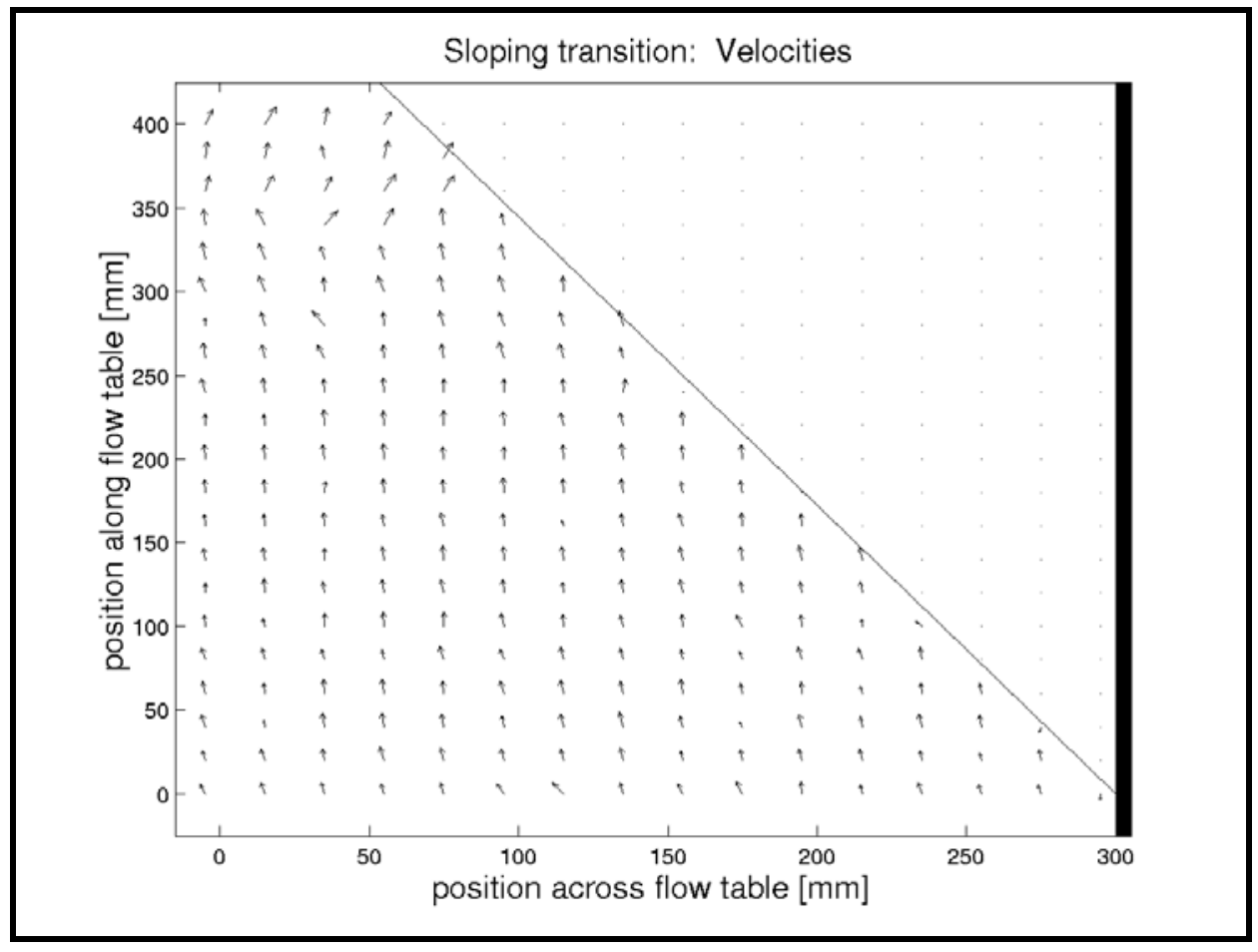

Figure 53. Flow leaving dredged region over sloping transition 


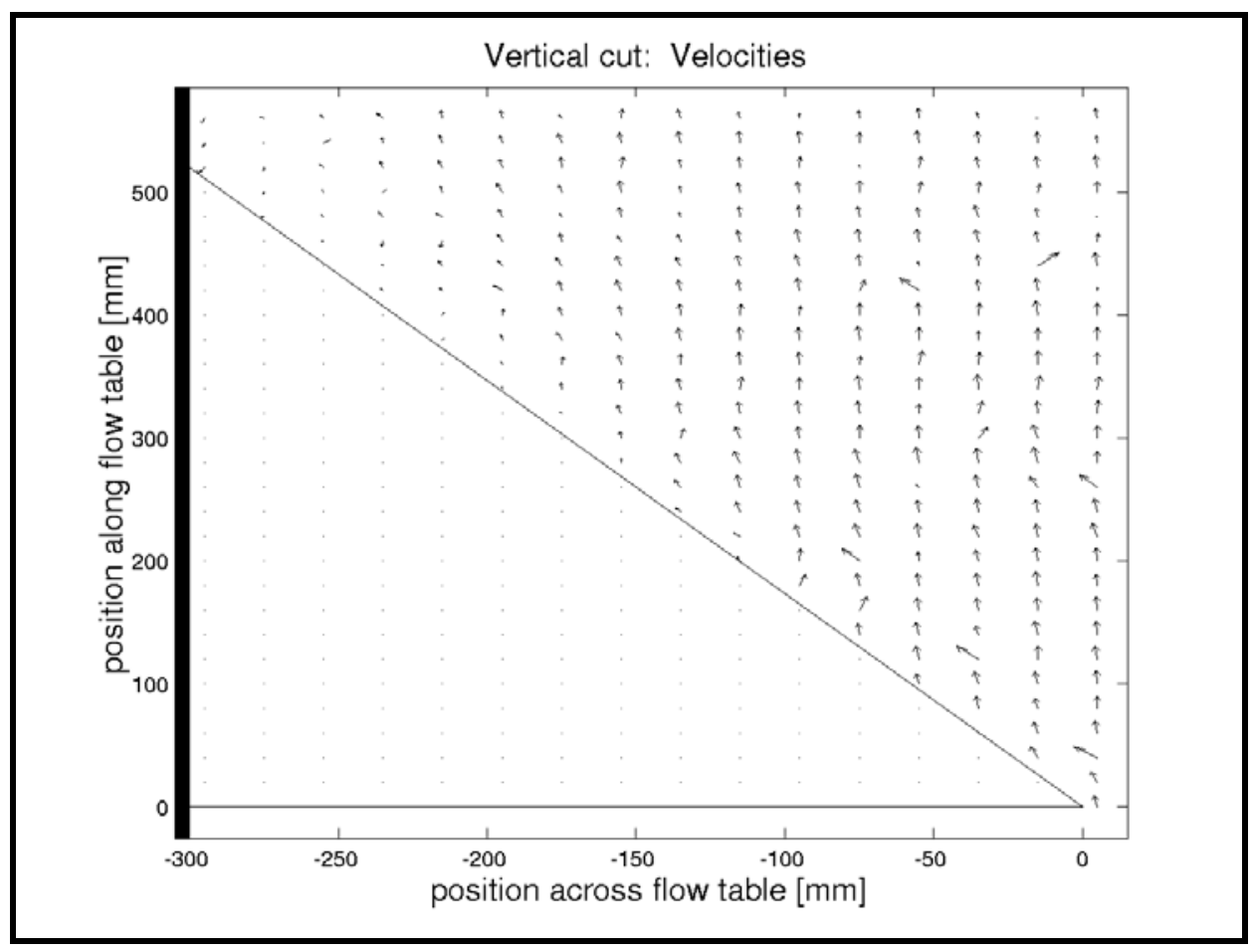

Figure 54. Flow entering dredged region over vertical transition

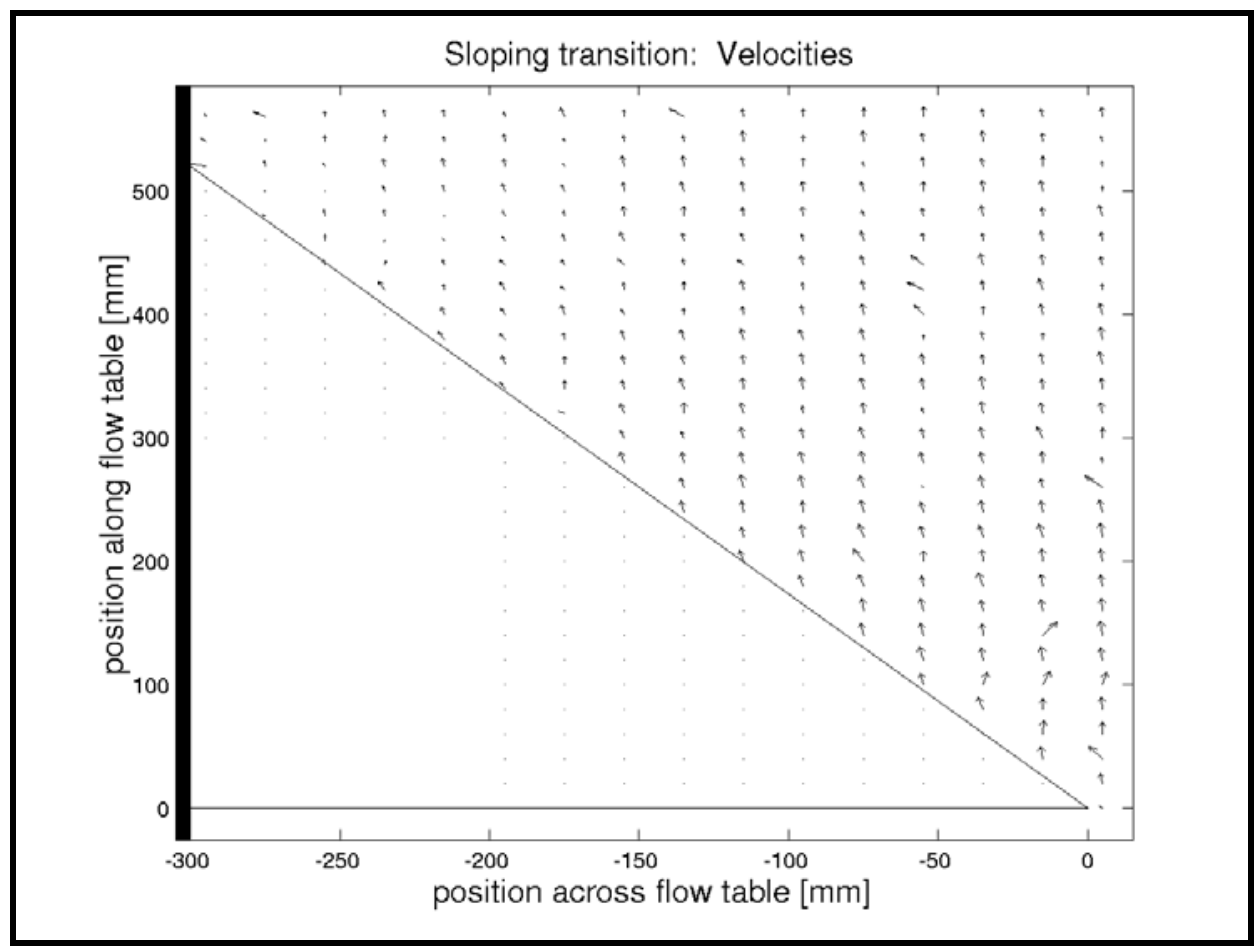

Figure 55. Flow entering dredged region over sloping transition 
structure in the lee of the step. Dye caught in the turbulent wake zone of the step moved laterally along the step in the direction of increasing step height. This is well illustrated by the dye concentrations in the photograph of Figure 56 where the higher vertical step is at the bottom of the image, and the dye moved from upper right to lower left in the photograph. The slower velocities in the turbulent region allow more time for suspended sediments to be deposited adjacent to the vertical cut.

There was no flow separation evident for the sloping transition (see Figure 55), and dye injected upstream of the transition moved straight into the dredged area without any substantial decrease in velocity as shown in Figure 57. Consequently, deposition of suspended sediment should be less because of decreased resident time in the harbor.

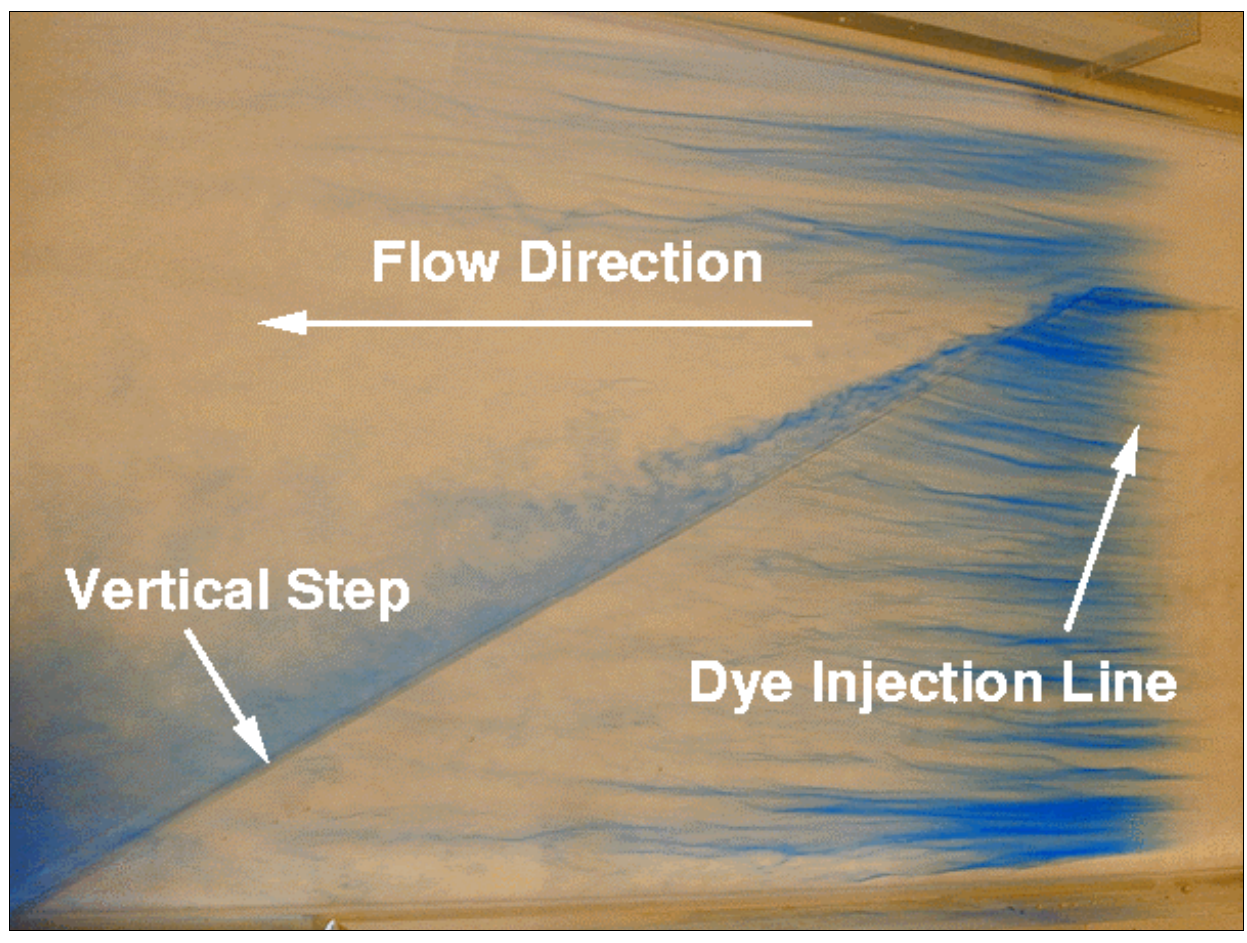

Figure 56. Photograph of flow entering dredged region over vertical transition 


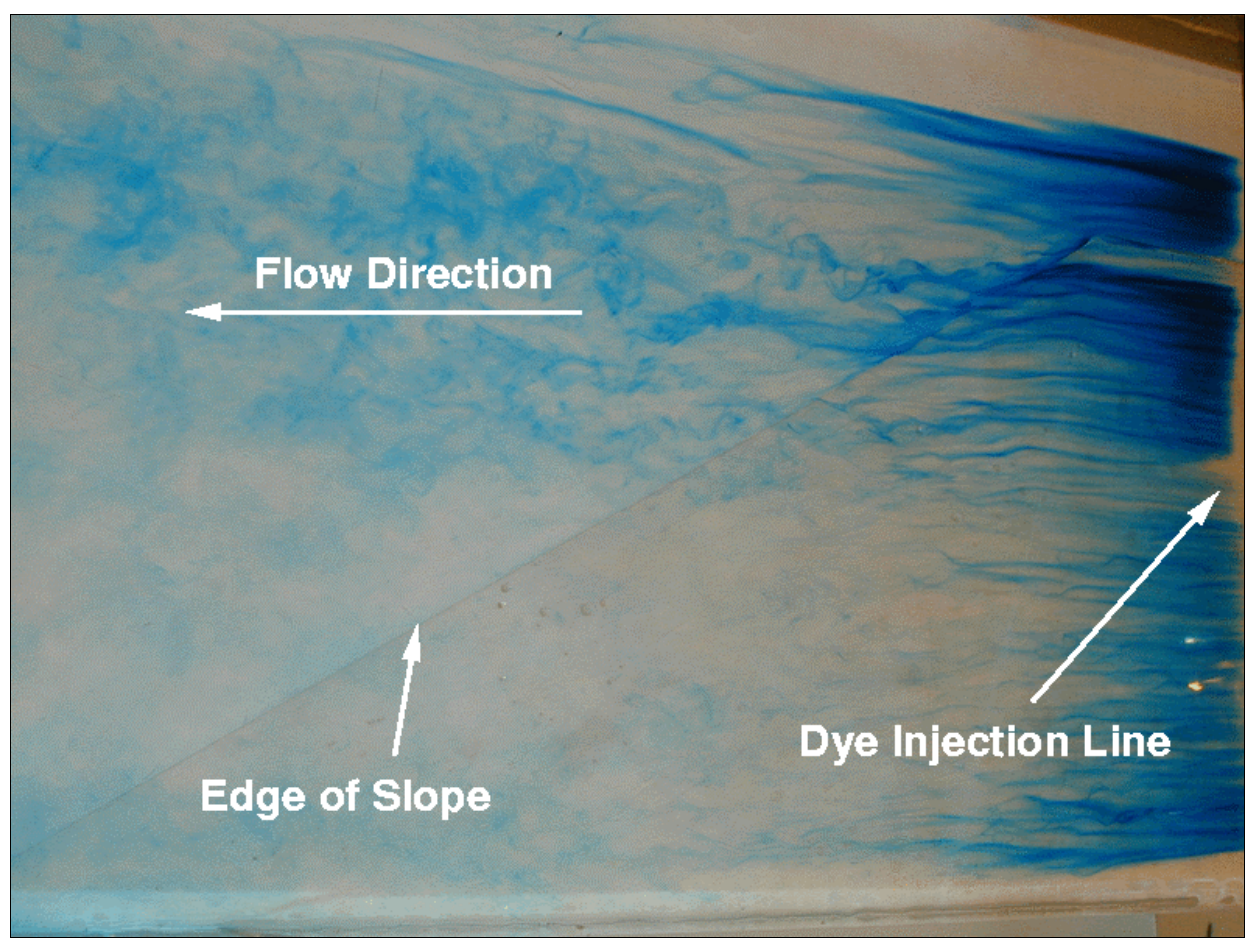

Figure 57. Photograph of flow entering dredged region over sloping transition

\section{Conclusions from Dredge Transition Experiments}

Tidal flow leaving the harbor across a vertical transition is redirected seaward closer to shore where the transition step is highest. The redirected velocity increased somewhat (up to $13 \mathrm{~cm} / \mathrm{sec} 5.1 \mathrm{in} . / \mathrm{sec}$ )), and this might even promote a scouring effect near the vertical cut. Flow leaving the harbor over a sloping transition remained straight and mostly uniform with magnitudes on the order of $11 \mathrm{~cm} / \mathrm{sec}(4.3 \mathrm{in} . / \mathrm{sec})$. Neither transition should cause an increase of suspended sediment deposition potential because flow velocities remain reasonably high over the transition.

Tidal flow entering the harbor across a vertical transition creates a turbulent wake region that has suspended sediment deposition potential in the immediate area downstream of the step. In addition, fluid is advected laterally along the vertical cut which would move sediment shoreward toward the mooring area. Replacing the vertical transition with a sloping transition significantly reduced the turbulent wake resulting in smoother flow patterns and less likelihood of sediment deposition. 


\section{3-D Cook Inlet Model}

During 7-9 May 2002, engineers from the Alaska District participated in experiments using the two idealized flow models. Results from the tests appeared to reproduce the primary flow patterns in the vicinity of the Port of Anchorage and identified the potential mechanism responsible for sediment deposition in the harbor. Everyone involved in the experiments agreed that a small-scale flow table model incorporating the actual 3-D bathymetry would provide more reliable flow patterns and greater understanding of the shoaling process. At the same time it was realized that extending the model coverage both upstream and downstream from the Port of Anchorage would better enable Alaska District engineers to assess potential remedies.

Additional funding was provided to construct and test a physical model of Upper Cook Inlet featuring 3-D bathymetry. The objective of the 3-D model was to identify and examine the large-scale flow patterns in upper Cook Inlet during maximum ebb and flood tide. A secondary objective was to investigate impacts related to various dredge release sites to optimize dredging operations.

\section{3-D Cook Inlet Model Description}

\section{Model scaling and bathymetry}

Figure 58 shows the portion of upper Cook Inlet that was scaled and reproduced for testing in the CHL precision flow table. The bounded rectangle in the figure measures approximately 18.2 by $51.2 \mathrm{~km}$ ( 11.3 by 31.8 miles) for a total area of about $932 \mathrm{sq} \mathrm{km} \mathrm{(360} \mathrm{square} \mathrm{miles).} \mathrm{The} \mathrm{shorter} \mathrm{dimension} \mathrm{was}$ scaled to fit within the 1.22-m (4-ft) width of the flow table. Using the same horizontal scale, the longer dimension scaled to $3.4 \mathrm{~m}(11.17 \mathrm{ft})$, which exceeded the 2.4-m (8-ft) length of the flow table, but gave the desired extended model coverage.

The longer dimension of the model was divided into six equal-length sections measuring $56.64 \mathrm{~cm}$ (22.3 in.) each. This provided the capability of testing any four adjacent sections in the flow table. For example, during ebb flow, a greater distance upstream from the Port of Anchorage could be placed on the flow table to assure better replication of the upstream boundary conditions. When the model was reversed in the flow table to simulate flood tide, the sections south of Fire Island could be included. This methodology proved to be useful for extending the region that could be examined using the flow table by adding and removing sections. 


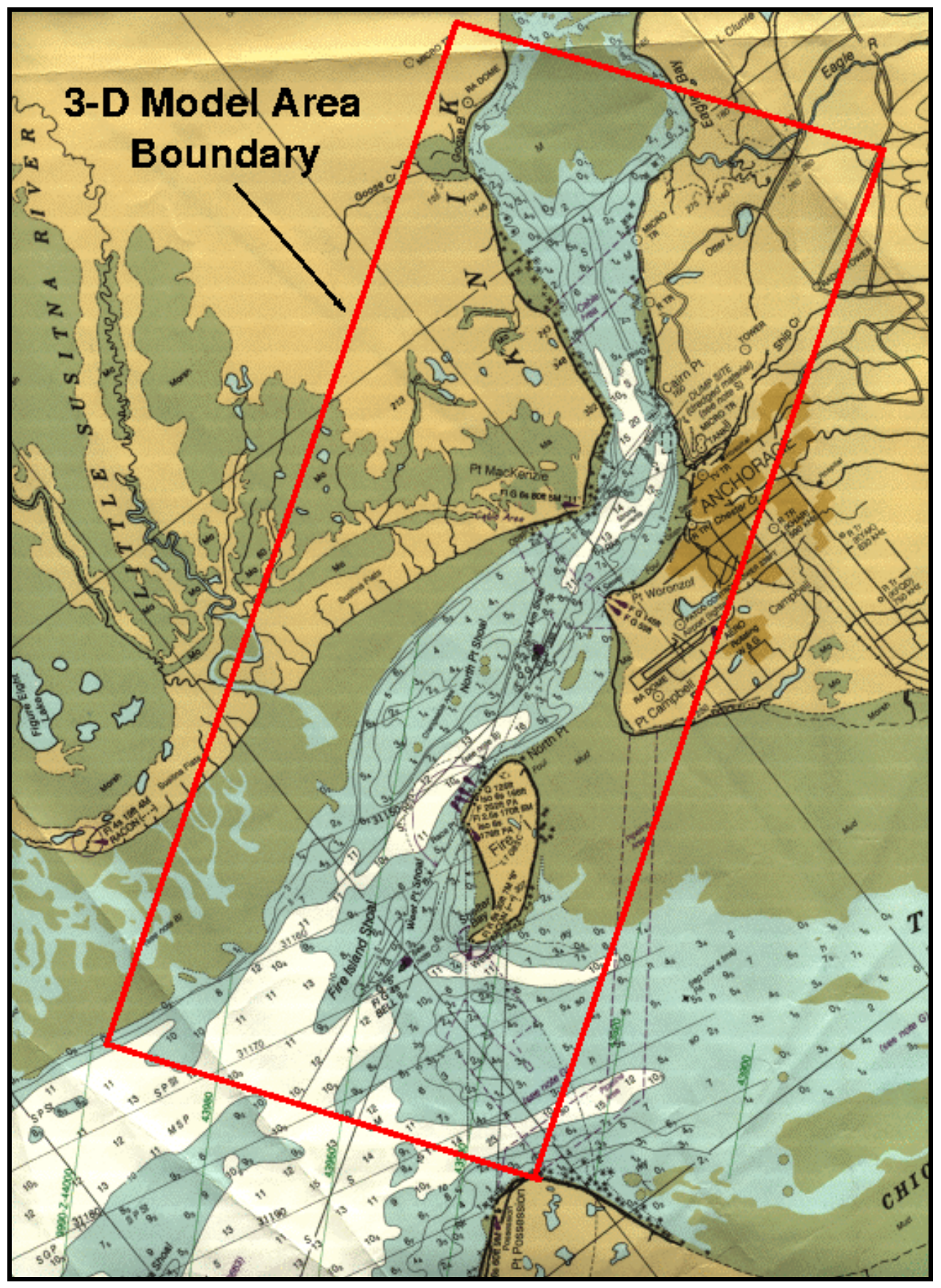

Figure 58. Area coverage of 3-D Cook Inlet model 
Bathymetry data for the selected region were obtained from a variety of sources and adjusted to a mean tide level $(\mathrm{mtl})$ datum. Survey data were used where available, otherwise elevations were obtained from National Oceanic and Atmospheric Administration (NOAA) Earth Topography Five Minute Grid (ETOP05) elevation data. Virtually no data existed for the extensive tidal flats defined as the area between the mean high water (mhw) and mean lower low (mllw). Because the available data showed reasonable agreement between mhw and mllw contours, the tidal flats (for the most part) were established as a linear interpolation between mhw and mllw.

Horizontal coordinates of the irregularly-spaced bathymetry data were expressed in latitude and longitude, and the corresponding vertical elevations were in units of meters relative to mean tide level $(\mathrm{mtl})$. Bathymetry over the modeled region represented the range between el -184 to $\mathrm{el}+10 \mathrm{ft}$ relative to $\mathrm{mtl}$. This total range was equivalent to $5.91 \mathrm{~cm}$ (2.33 in.) in the model. It was necessary to add vertical shoreline banks to the carved model to accomodate tide levels above el $+10 \mathrm{mtl}$. Relevant scaling factors (prototype-to-model ratios) for the 3-D model are listed in Table 10 along with approximate model equivalences.

\section{Table 10}

\section{Scale Ratios for 3-D Model}

\begin{tabular}{||l|l|l||}
\hline \hline Scale & Scale Value & Model Equivalence \\
\hline \hline Horizontal Scale & $N_{X}=15,000$ & $1,250 \mathrm{ft} \approx 1 \mathrm{in}$. \\
\hline Vertical Scale & $N_{Z}=1,000$ & $83 \mathrm{ft}=1 \mathrm{in}$. \\
\hline Velocity Scale & $N_{V}=\sqrt{N_{Z}}=31.6$ & $1.6 \mathrm{~m} / \mathrm{sec}=5 \mathrm{~cm} / \mathrm{sec}$ \\
\hline Discharge Scale & $N_{Q}=N_{X} N_{Z}^{3 / 2}=474,341,650$ & $203,000 \mathrm{cu} \mathrm{m} / \mathrm{sec}=0.43 \mathrm{~L} / \mathrm{sec}$ \\
\hline
\end{tabular}

The prototype-scale irregularly-spaced bathymetry was first converted to State Plane coordinates for Alaska Zone 4: 5004, and then interpolated onto a $240 \times 1072$ uniform grid containing just over one quarter of a million points. The data were then scaled to model dimensions (inches) by applying the appropriate scale and unit conversion factors using a Fortran program. In model dimensions, the points were spaced at one-fourth inch increments along a line going across the 1.22-m (4-ft) length, and the 1,072 lines spanning the 3.4-m (134-in.) length were at one-eighth inch spacings. Figure 59 shows a 3-D rendition of the scaled model. There is no vertical exaggeration in this image (other than the model distortion). Digital bathymetry files were transferred to the Model Shop's computer, converted into 3-D surfaces, and then translated into machine path instructions. 


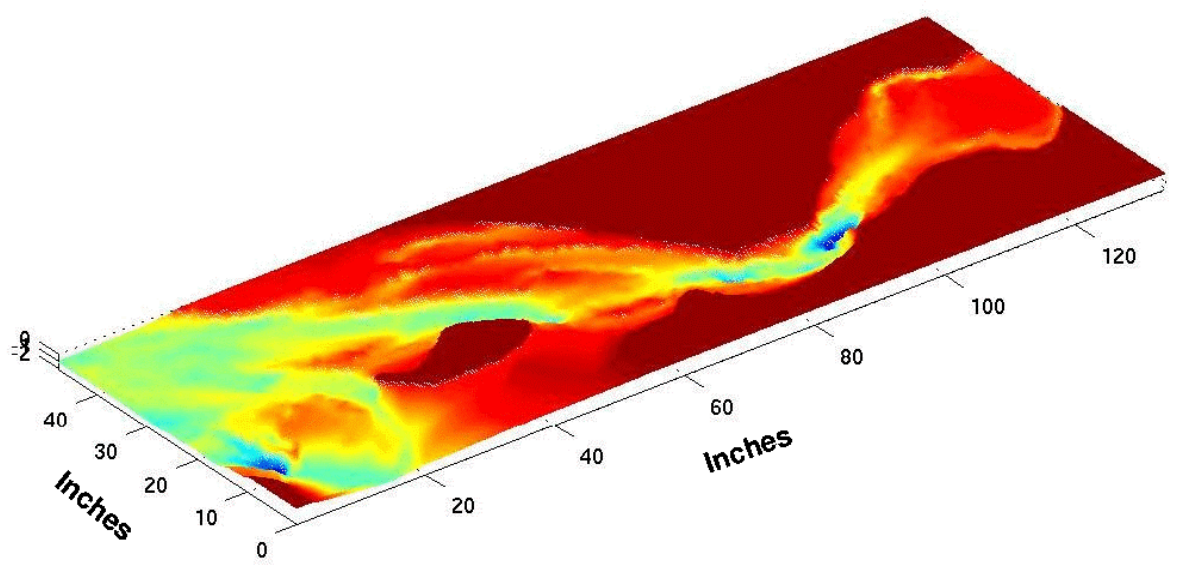

Figure 59. Bathymetry of 3-D Cook Inlet model

\section{Model fabrication}

The Cook Inlet 3-D model was carved into Plexiglas using the programmable router discussed in Chapter 3 and illustrated in Figure 8. Sheets of 2.54-cm-

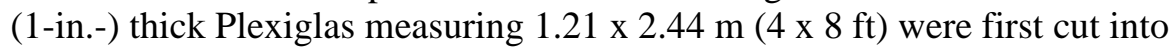
smaller pieces measuring $56.64 \times 121.9 \mathrm{~cm}$ (22.3 x 48 in.), and then three pieces were laminated together to form a model section with a total thickness of $7.62 \mathrm{~cm}$ ( 3 in.). The 121.9-cm (48-in.) width was then cut into two pieces and the edges trimmed up. In other words, the model consisted of 12 blocks measuring approximately 60.20 x $56.64 \mathrm{~cm}(23.7 \times 22.3$ in.) arranged into a 2 x 6 matrix corresponding to the overall model dimensions. As mentioned earlier, the primary reason for sectioning the model was to allow different reaches of the study area to be placed on the flow table. The additional sectioning down the middle of the long axis of the model was needed for the practical reason of being able to move the blocks in and out of the flow table as needed without special hoists. Each block, before carving, weighed about $320.3 \mathrm{~N}$ (72 lb), which is easily maneuvered by two people. Blocks weighed less after the bathymetry was cut.

A test cut was made into high density Styrofoam to determine the optimum step-over distance for each traverse of the finish milling bit. A value of about one one-hundredth of an inch provided smooth results. Next, eight of the Plexiglas blocks were jigged up on the router table, and a large bit was used to mill the upper surface of the blocks to perfect level. (There are slight variations in thickness of the Plexiglas sheets.)

Cutting of the bathymetry for each section proceeded in two steps. First, the bathymetry was "rough-cut" using a larger bit down to about one-sixteenth inch above the final elevation. Figure 60 shows the rough-cut on the first section on the south end of the model. The rough-cut proceeded quickly because the bit chews out about one-fourth inch of depth with each pass. 


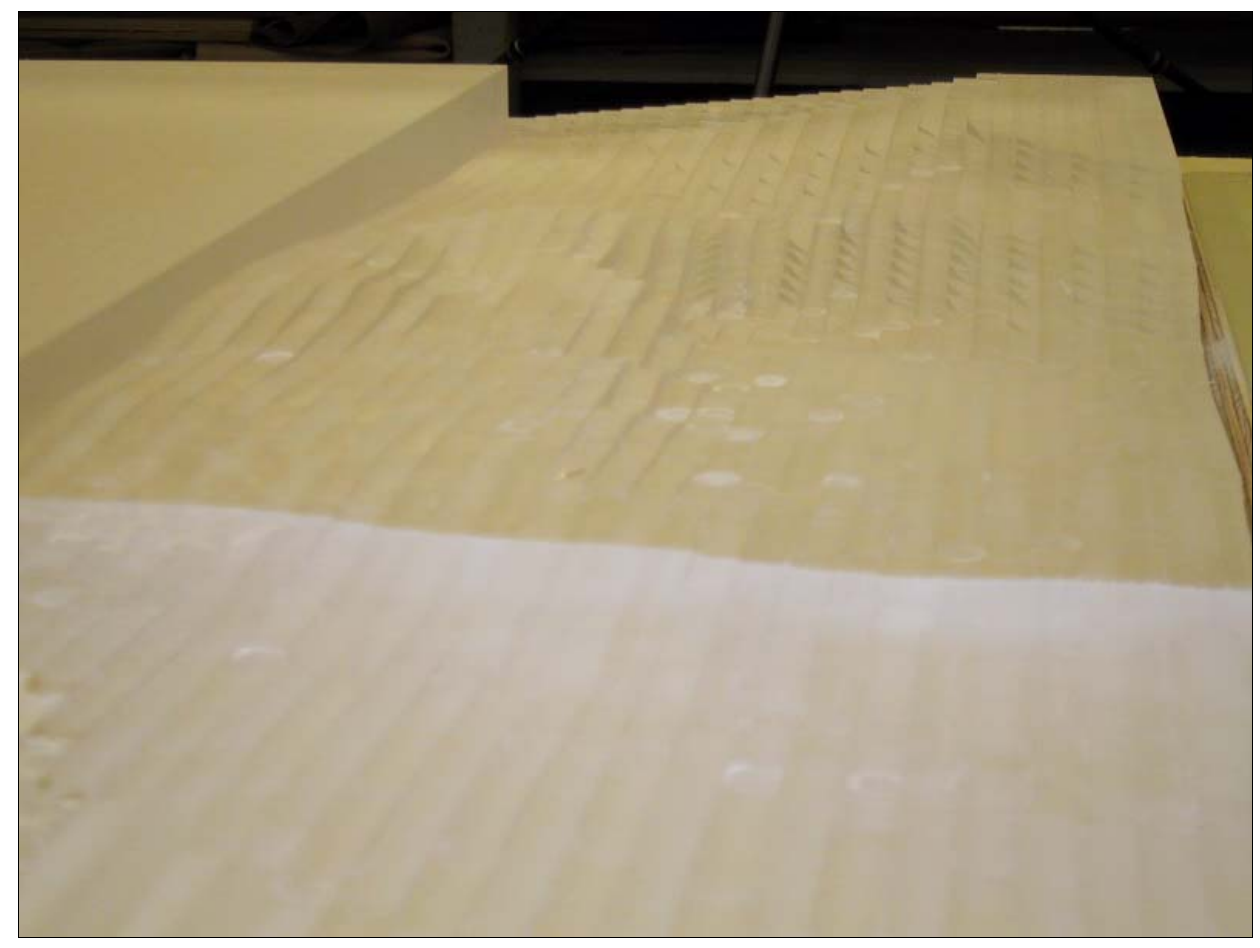

Figure 60. Router rough-cut of bathymetry

A smaller ball-head bit was used to "finish cut" the section. This proceeded at a much slower rate that varied depending on depth of bathymetry and how rapidly the depths changed. For sections that required cutting across the entire $1.22-\mathrm{m}$ (4-ft) width, the finish cut progressed along the long dimension of the model at about $3.81 \mathrm{~cm} / \mathrm{hr}(1.5 \mathrm{in} . / \mathrm{hr})$. This rate improved on sections of the model where the channel was narrower and only covered a small portion of the 1.22-m (4-ft) width. Figure 61 shows the finish cut complete for the first section and in progress on the second section.

After the entire model was carved, the milled surface of each section was polished, and the vertical shorelines were cut and added to the model. Figure 62 shows a portion of the completed model looking upstream from a viewpoint south of Fire Island. (Notice the vertical shorelines being added to the model.) Figure 63 is a view looking southward toward Cairn Point. The vertical shorelines had not been added when this photograph was made. Close-up views of the model bathymetry are shown in Figures 64 and 65. Keep in mind this model has a 1:15 model distortion, so the actual horizontal distances in the prototype are stretched out relative to the depth, and the slopes are much milder. 


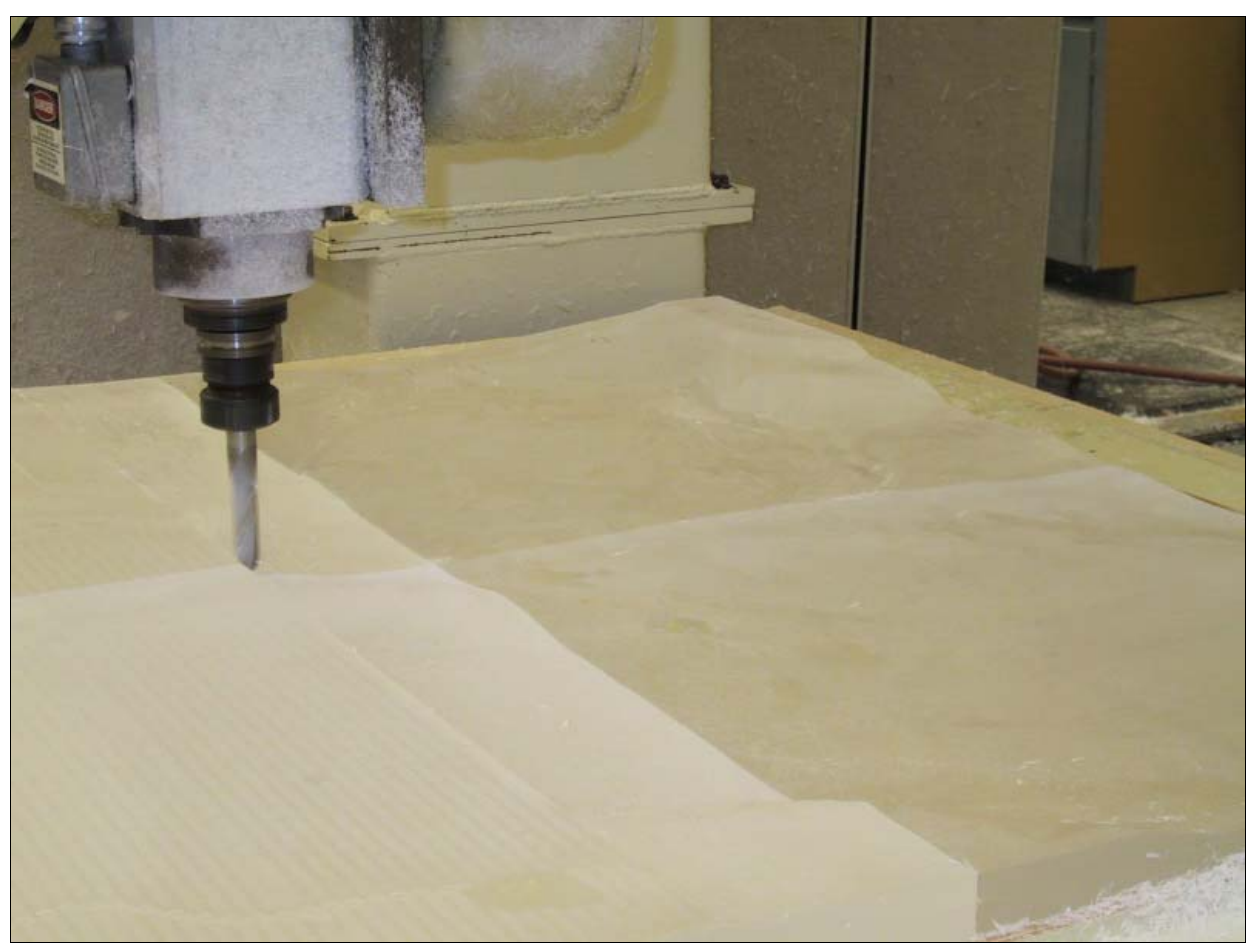

Figure 61. Router finish cut of bathymetry

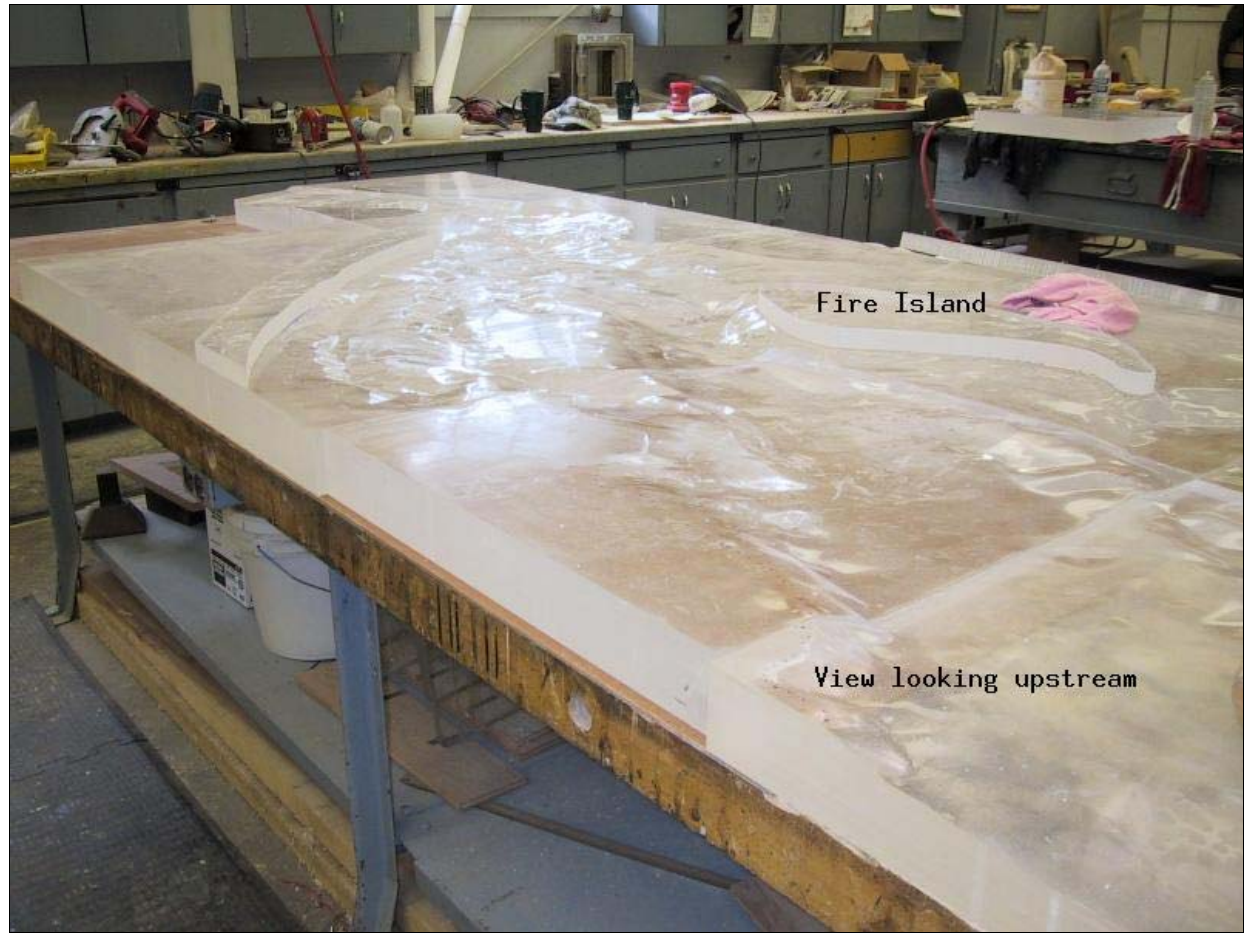

Figure 62. Portion of 3-D model looking upstream 


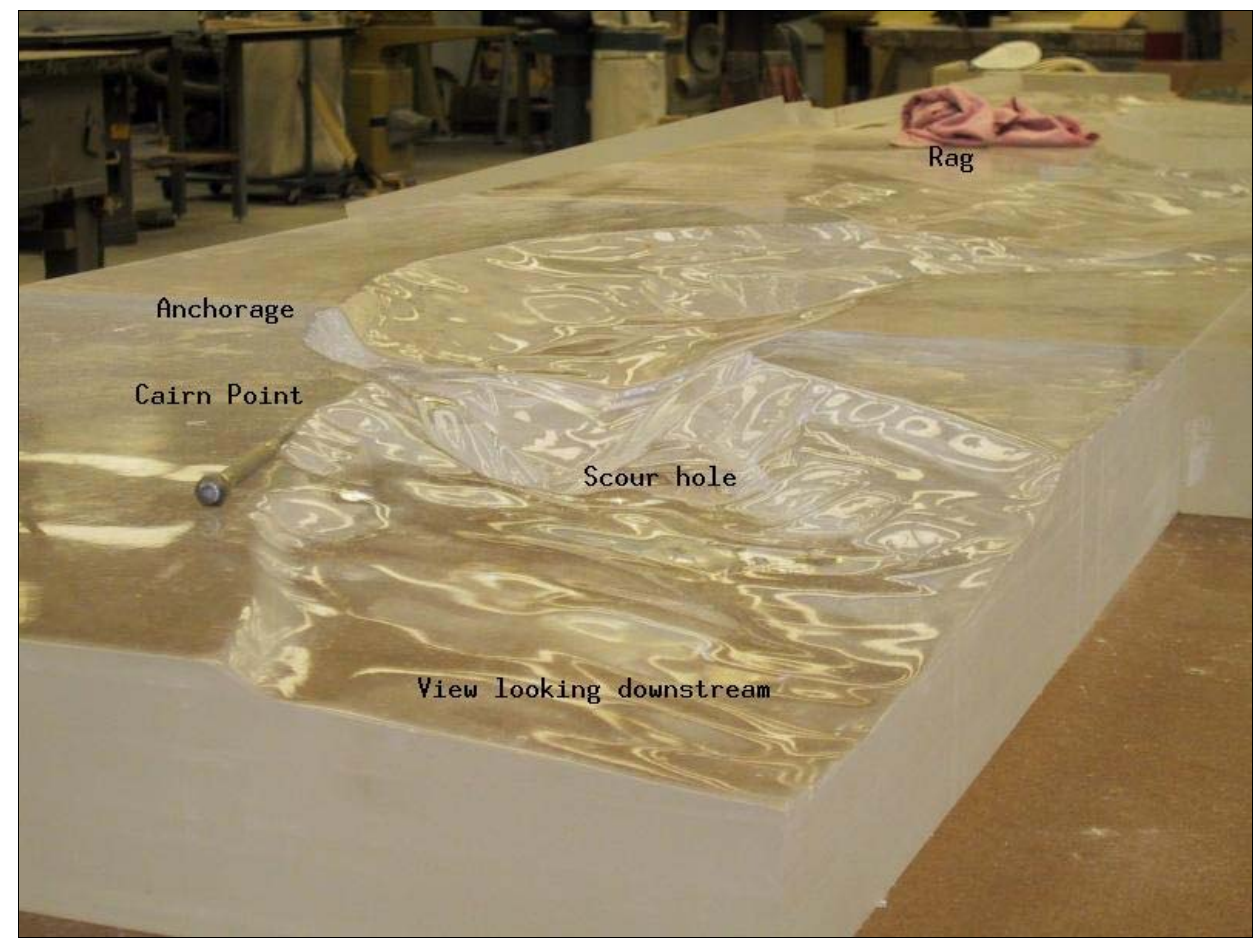

Figure 63. Portion of 3-D model looking downstream

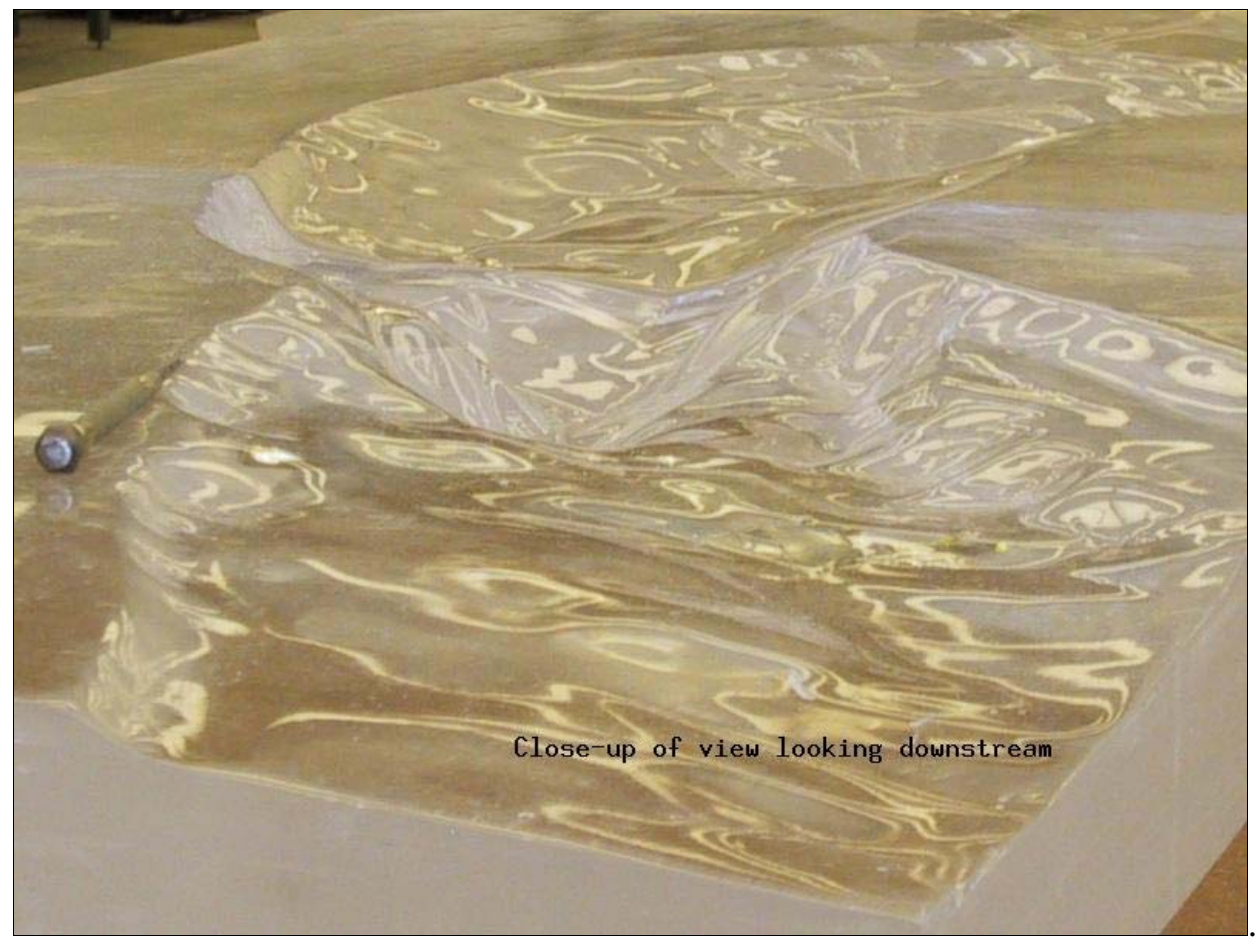

Figure 64. Close-up view looking downstream at Cairn Point 


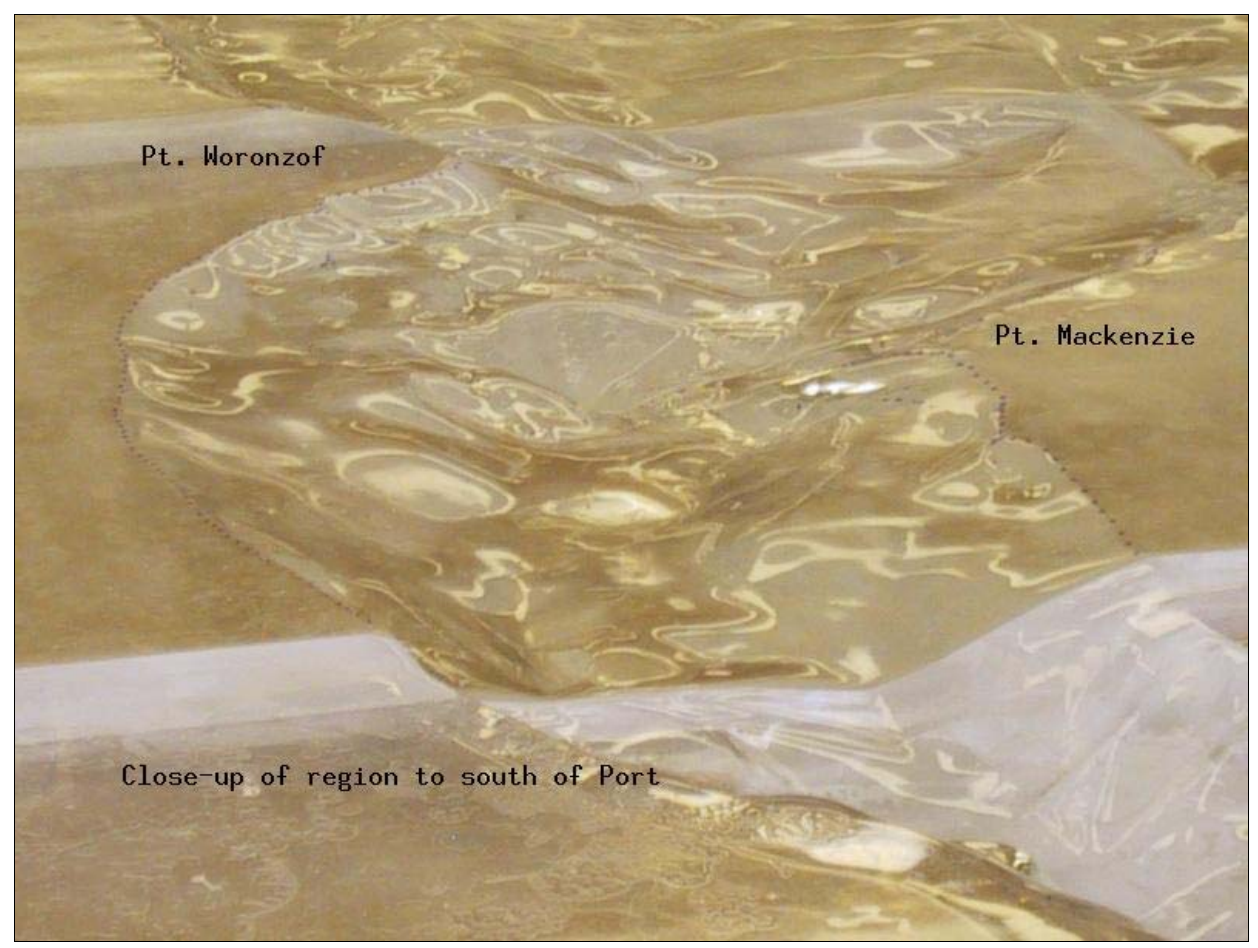

Figure 65. Close-up view looking downstream south of Port of Anchorage

\section{Model operation}

Operation of the 3-D Cook Inlet model is essentially the same as for the idealized models discussed in Chapter 3. Four sections of the six-section model were placed on the flow table as illustrated in Figure 66, and the water level was adjusted for a specified tide elevation (usually mhw or mtl) using the adjustable weir located at the downstream end of the flow table. After turning on the circulation pump, the steady flow rate was adjusted to the scaled discharge value representing the target total discharge at Cook Inlet. (Simulation of a variableflow tide hydrograph was not supported at this time.)

Flow visualization techniques using dye injection and tracers (surface and bottom) were used to determine regions of flow separation, flow entrainment, and reduced-flow areas where fine sediment would be deposited. The dye revealed the 3-D nature of the turbulence, and was injected using different sized syringes. One method was to squirt a line of dye across the principle flow direction to observe how it translated downstream. A second method was to continually introduce dye at an upstream point so a pathline formed as the dye moved downstream. Baby powder worked well as a tracer.

Changing between ebb and flood tide was accomplished by rotating the entire model by $180 \mathrm{deg}$ on the flow table so the water flowed in the opposite direction. The region of interest was changed by removing a section on one end and adding a section on the opposite end. The effect of shoal migration was examined by moving existing shoals or creating new shoals using Silly Putty ${ }^{\mathrm{TM}}$ or floral clay. 


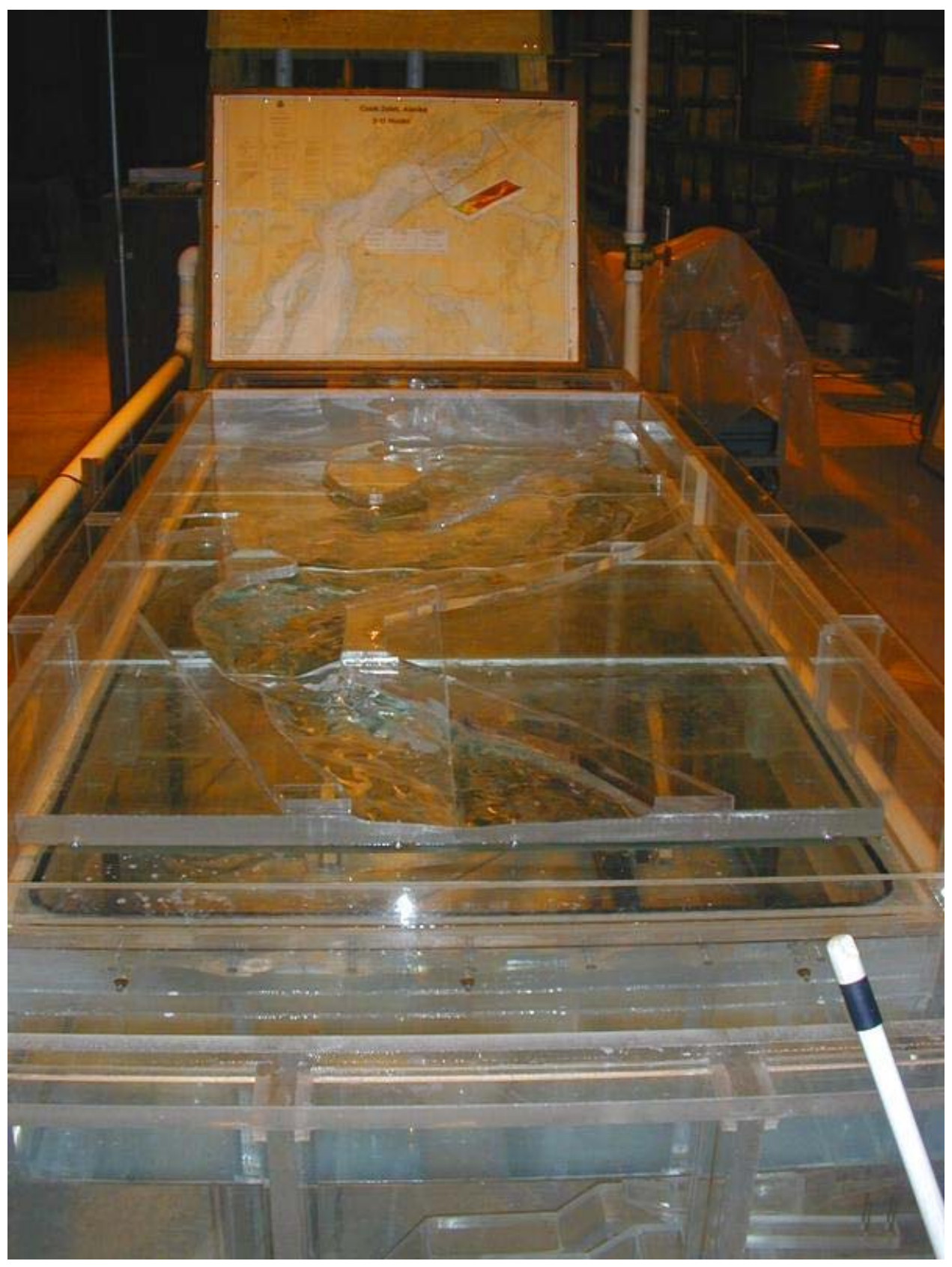

Figure 66. 3-D Cook Inlet model installed for flood flow 


\section{3-D Model Observations}

During the week of 4-7 November 2002, engineers from the Alaska District participated in tests using the 3-D Cook Inlet flow table model. All of the largescale flow structures observed in the idealized models during flood and ebb tide were seen in the 3-D model. This included flow separation at the major headlands (Point Woronzof, Point MacKenzie, and Cairn Point), large slowmoving gyres in the lee of the headlands, and areas of ebb-flow reduction at the Port of Anchorage.

Previously, flood flow tests in the idealized models indicated a strong crosschannel bottom flow originating just north of Point Woronzof and crossing the inlet diagonally toward Port MacKenzie. The mechanism for this transport is local flow acceleration and flow separation at Point Mackenzie which decreases

the local water elevation and creates a cross-channel momentum inbalance that is alleviated by cross-channel mass transport at the bottom. During the idealized model tests it was hypothesized that the vertical sides of the different depth terraces might unrealistically be contributing to the cross-channel flow. However, the same cross-channel transport occurred in the 3-D model at approximately the same location as observed in the idealized model. In the 3-D model it seemed that the cross-channel flow was weaker, but this may have been the result of reduced model discharge used in the 3-D model.

Impacts related to shifting of the Fire Island shoals were examined by placing new shoals fabricated out of floral clay into the main channel on the west side of Fire Island. This modified the local flow patterns, but no detectable changes seemed to occur farther upstream in the vicinity of the Port of Anchorage. However, the shifting shoals might create conditions suitable for additional entrainment of fine sediment from the mud flats which in turn could result in increased dredging requirements at the Port of Anchorage.

A key discovery of the idealized model tests was the formation of an area of reduced flow velocity in the lee of Cairn Point during ebb tide. This was thought to be the primary cause for siltation at the Port of Anchorage. Simulation of maximum ebb flow over the actual bathymetry of the 3-D model generated the same area of reduced flow at the Port of Anchorage as was observed in the idealized models. Figure 67 shows ebb currents moving surface tracers past Cairn Point. Most of the tracer has already moved downstream with the exception of the tracer particles caught in the gyre formed in the lee of Cairn Point. Within the gyre the tracer particles slowly circulated in a counterclockwise direction. Fine sediment entrained in the water would have ample opportunity to deposit on the bottom.

Dye injected near the bottom in the reduced-flow region adjacent to the Port of Anchorage also exhibited lengthy residence times before moving on downstream as shown in Figure 68. Dye injected upstream of Cairn Point near the east bank of the inlet ended up being trapped in the gyre south of the point. The extent of flow separation and entrainment at Cairn Point during ebb flow was estimated and sketched on the aerial photograph shown in Figure 69. During flood flow in the model, surface tracers and subsurface dye moved freely northward past the port indicating that the volume of deposited sediment would be significantly less during flood tide. 


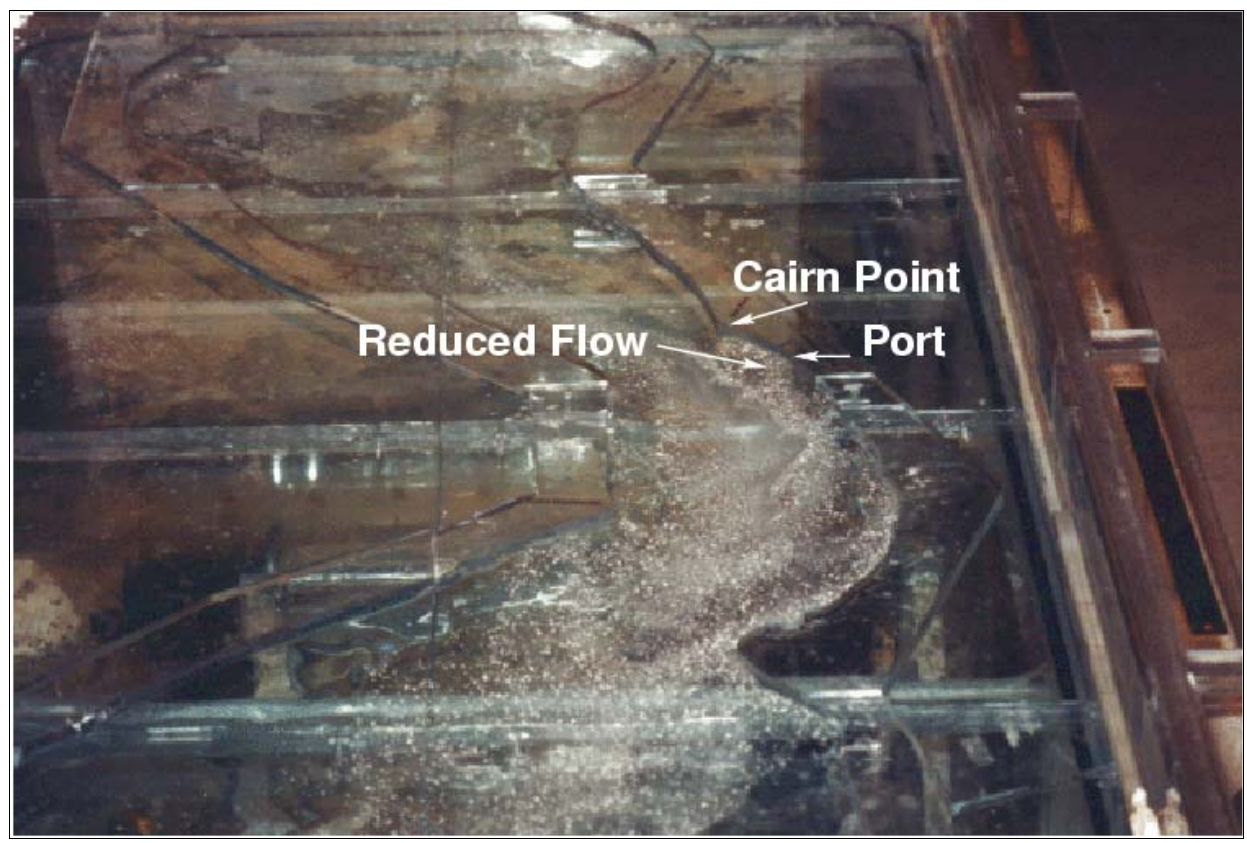

Figure 67. Surface flow tracer showing reduced flow at Port of Anchorage during ebb tide

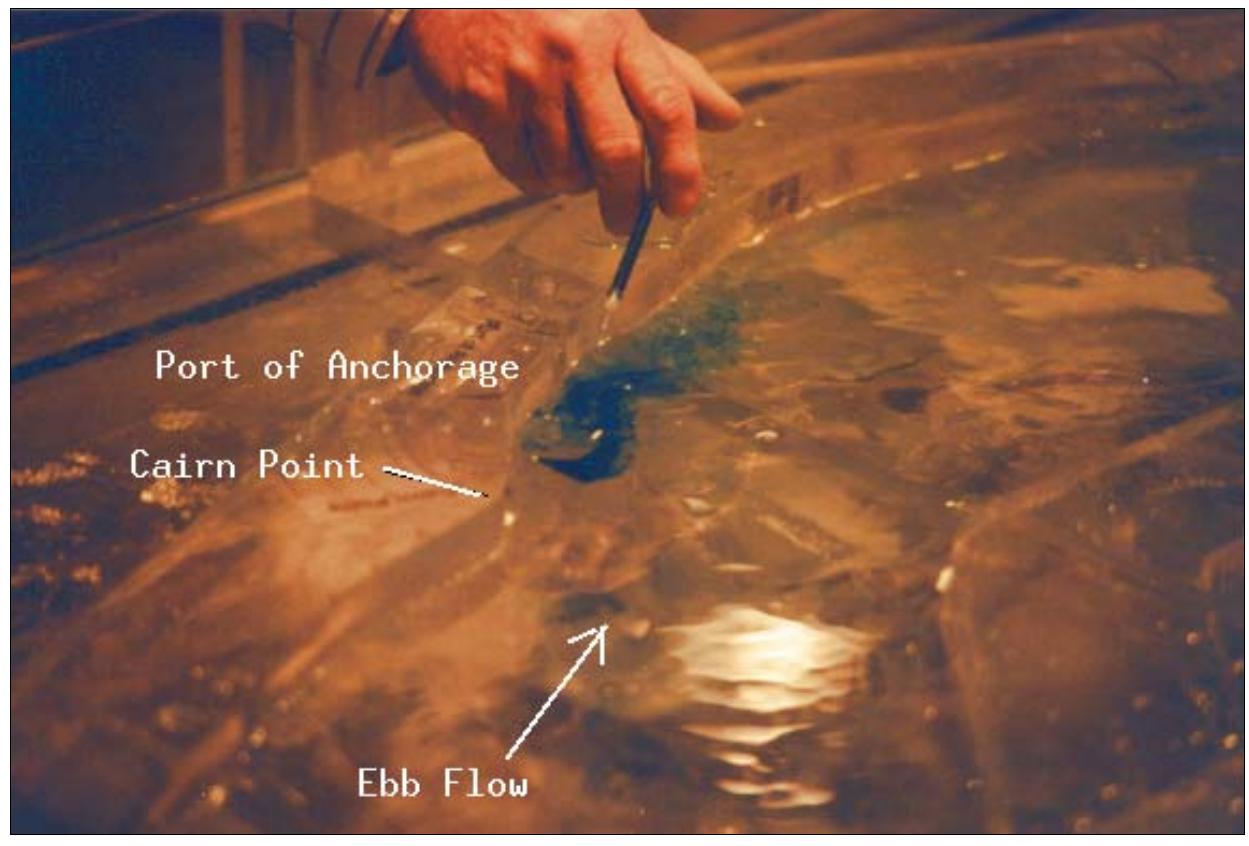

Figure 68. Dye injection showing reduced flow at Port of Anchorage downstream of Cairn Point 


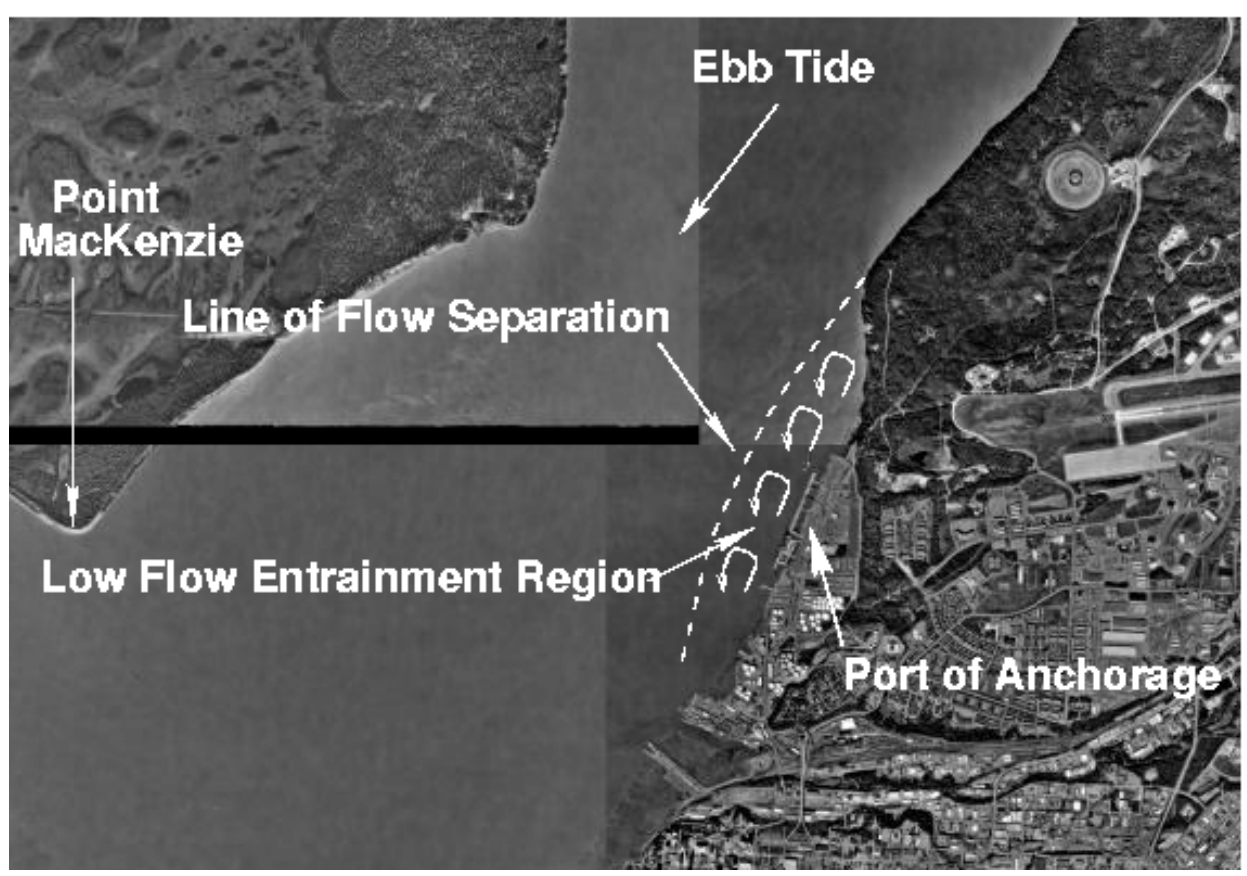

Figure 69. Aerial showing approximate flow separation and entrainment region in lee of Cairn Point during ebb tide

The Alaska District engineers used dye injection to investigate how dredged sediment might move when deposited from barges at different locations during both ebb and flood flows. Approximate location of established dump sites were scaled on the model, and dye was slowly injected at various depths in the water column. Depending on the flow direction and the injection point, it was not unusual to observe dye migrating back into the vicinity of the port. Movement of the dye injection location, or depositing during the opposite tide flow often alleviated this problem. Floral clay was used to simulate dredged areas within the Port of Anchorage. Dye injections indicated that dredging near the dock face without providing a dredged pathway out to the main channel tended to trap dye within the harbor area. The amount of trapped dye was lessened by opening of a dredged channel. Through these simple experiments the Alaska District will be able to recommend dredging and disposal practices that should substantially reduce the amount of deposited material re-entering the port area. Cost savings associated with more efficient dredging practices could be significant.

Floral clay was also used to approximate a proposed 12.2-m (40-ft) expansion of the dock into the channel. This small protrusion had minimal detectable effect on the predominant flow patterns in the vicinity of the Port of Anchorage.

Finally, a simple, qualitative test was performed to examine impacts of boundary friction in the physical model. The Plexiglas model had a smooth surface, and there was a potential scale effect arising from insufficient roughness on the model boundaries. The boundary must be rough enough to initiate a fully turbulent boundary layer extending from just above the bottom all the way to the flow free surface. Ink injected directly onto the Plexiglas exhibited laminar boundary layer characteristics, but higher up in the water column the flow was obviously very turbulent. 
The main concern related to incorrect boundary friction is flow moving around bends where the centrifugal acceleration is countered in part by bottom friction. After observing flow characteristics for a flood flow simulation, the model was dried, and a coat of rubber cement was applied to the Plexiglas in a rough, thick layer with cross-channel brush strokes. When dried, the glue layer provided a rough surface. Observations of flow patterns with the roughened boundary were not noticeably different from those previously observed.

However, it is important to note this was not a rigorous test, and this issue should be revisited in a more controlled test using measurements from the laser Doppler system.

\section{Conclusions from Cook Inlet 3-D Model}

The 3-D model of Cook Inlet featured actual bathymetry reproduced at very small scale and with significant geometric distortion. Nevertheless, the largescale flow features such as flow separation at major headlands and gyre formations resembled known flow behavior observed at the study site. The capability of extending the study area in both directions by adding and removing sections enabled more replication of the critical upstream boundary conditions, thus assuring better simulation of flow conditions in the region of interest. The conclusions drawn from the 3-D model task are as follows:

a. Tidal flow in upper Cook Inlet features large, 3-D, gyres formed by flow separation at major headlands. The gyres exhibited vertical and lateral mixing, and regions of reduced flow velocity magnitude corresponded well with shoal areas observed in the prototype.

b. Turbulent flow separation at Cairn Point during ebb tide was shown to be instrumental in causing shoaling of the Port of Anchorage. This shoaling mechanism was first observed in the idealized models, but there was concern that the terracing of bathymetry in the idealized models might be contributing to the flow pattern. The 3-D model confirmed this was not the case.

c. Dye injection during flood and ebb tide demonstrated that dredge disposal practices could be improved by choice of disposal sites and timing dump releases to correspond with tidal flow direction. This reduces the possibility of dredged material being immediately carried back into the harbor area.

$d$. Dredging along the dock face without leaving a pathway to the deeper channel could promote trapping of suspended sediment and increased sedimentation in the harbor region.

e. Modifying the Fire Island shoals did not appear to affect flow patterns farther upstream around Anchorage during flood tide. However, this conclusion is based only on visual observation without any quantifying measurements.

$f$. The smooth boundaries of the physical model might impede development of fully turbulent flow, and thus represent a scale effect. However, addition of surface roughness did not have any impact on the overall flow conditions observed in the model. This may be the result of 
the water passing through a perforated screen before flowing across the table, making the flow somewhat turbulent initially.

g. The value of small-scale, 3-D physical models was amply demonstrated by Alaska District engineers who used the model to understand more fully some of the reasons for sedimentation at the Port of Anchorage, and to develop cost-saving dredging procedures at the port. Being able to visualize the flow with dyes and tracers provided insight and instant feedback to enhance their understanding about the primary flow processes.

$h$. Although 3-D flow structures were evident throughout the flow regime, the turbulent vertical velocities did not seem as important as the horizontal motion of the gyres. Therefore, 2-D, depth-averaged numerical models might provide reasonable approximation of the overall flow in Cook Inlet. However, details of motion within the gyres or flow entrainment areas are best investigated using a physical modeling approach, preferrably with minimum geometric distortion. 


\section{Summary and Conclusions}

The U.S. Army Engineer District, Alaska, funded studies involving the new CHL Precision Flow Table. The studies provided insight into the complex flow regime of upper Cook Inlet, and the small-scale models of Cook Inlet illustrated the probable shoaling mechanism at the Port of Anchorage. In addition, generic flow table studies of flow separation and large-scale turbulence generation examined potential scale effects that may occur in geometrically distorted physical models. Results from the scale effects experiment will factor into any decisions to construct a large physical model of Cook Inlet.

\section{Summary of Study Tasks}

The five tasks undertaken for this study are summarized in the following paragraphs.

\section{Task 1: Large-area idealized flow model of Cook Inlet}

This model covered a larger region of Cook Inlet than the small-area model, and the bathymetry was idealized with two horizontal surfaces at elevations corresponding to $0 \mathrm{ft}$ and $-60 \mathrm{ft}$ mllw. The objective of the model was to identify and examine in a semiquantitative way the large-scale flow patterns in upper Cook Inlet during maximum ebb and flood tide.

The large-area idealized physical model of Cook Inlet proved to be valuable in understanding the complex flow patterns in the vicinity of Anchorage, AK. Even though the model was very small, constructed at a highly distorted geometric scale, and represented fairly complex bathymetry by simple horizontal terraces, it still produced large-scale turbulent flow features known to exist in upper Cook Inlet. Furthermore, the location and areal extent of the gyres and eddies were qualitatively the same as in the prototype as confirmed by Alaska District engineers.

\section{Task 2: Small-area idealized flow model of Cook Inlet}

This model was similar to the large-area idealized model, but it covered a smaller area in the vicinity of the Port of Anchorage. In addition, bathymetry was idealized using three terraces rather than two. The objective of the smallarea model was to study in greater detail flow patterns identified in the large-area model in terms of potential contribution to sedimentation at the Port of 
Anchorage. The small-area idealized model also provided valuable insight into the tidal flow regime near the port. Observations made in these two idealized models were the stimulus for constructing a flow table model incorporating 3-D bathymetry.

\section{Task 3: Turbulence scale effect in distorted physical models}

A theoretical analysis and an extensive set of experiments were performed to assess potential turbulence scale effects that might arise due to flow separation and associated turbulence in geometrically distorted physical models. Results from these experiments will assist in determining whether a large-scale distorted physical model of Cook Inlet will produce reliable and useful results in regions where flow separation and turbulence are contributing to sedimentation.

Four distinct cases were examined: (a) Flow separation at a vertical edge resulting in a free jet, (b) Flow separation at a vertical edge with a constrained jet, (c) Flow separation at sloping edge resulting in a free jet, and (d) Flow separation at a vertical step. In all cases differences due to model distortion were evaluated by comparing the measured velocity fields of the prototype experiment with the velocity fields of the distorted models scaled to prototype size using appropriate scale ratios.

\section{Task 4: Effect of sloping transitions on flow hydrodynamics}

The objective of this task was to test the hypothesis that sloping transitions on both ends of the harbor would decrease areas of reduced flow and increase flushing of the harbor. Tests were conducted using vertical and sloping transitions between deeper dredged areas and the shallower adjacent channel profile. Both transitions were tested for the cases of flow leaving the harbor area and flow entering the harbor. The relative effect of the transitions on the flow was quantified with velocity measurements, and dye injections provided visual qualitative assessment.

\section{Task 5: Large-area 3-D flow model of Cook Inlet}

The objective of this task was to identify and examine in a 3-D flow table model the large-scale flow patterns in upper Cook Inlet during maximum ebb and flood tide. The 3-D model of Cook Inlet featured actual bathymetry reproduced at very small scale and with significant geometric distortion. Nevertheless, the large-scale flow features such as flow separation at major headlands and gyre formations resembled known flow behavior observed at the study site by Alaska District engineers. The capability of extending the study area in both directions by adding and removing sections enabled more replication of the critical upstream boundary conditions, thus assuring better simulation of flow conditions in the region of interest. The 3-D model was also used to examine potential sites for dredged sediment disposal that minimized risk of sediment moving back into the harbor. 


\section{Study Conclusions}

Major conclusions from the study tasks are presented in this section. The first group of itemized conclusions pertains to those tasks specific to Cook Inlet and problems with shoaling at the Port of Anchorage. The second set of conclusions relate to the study of turbulence scale effects in geometrically distorted physical models.

\section{Cook Inlet study conclusions}

a. Tidal flow in upper Cook Inlet features large, 3-D, gyres formed by flow separation at major headlands. The gyres exhibited vertical and lateral mixing, and regions of reduced flow velocity magnitude corresponded well with shoal areas.

$b$. Turbulent flow separation at Cairn Point during ebb tide was shown to be potentially instrumental in causing shoaling of the Port of Anchorage. This shoaling mechanism was first observed in the idealized models, but there was concern that the terracing of bathymetry in the idealized models might be contributing to the flow pattern. The 3-D model confirmed this was not the case.

c. Dye injection during flood and ebb tide demonstrated that dredge disposal practices could be improved by choice of disposal sites and timing dump releases to correspond with tidal flow direction. This reduces the possibility of dredged material being immediately carried back into the harbor area.

$d$. Dredging along the dock face without leaving a pathway to the deeper channel could promote trapping of suspended sediment and increased sedimentation in the harbor region.

$e$. Upstream boundaries are very important to the flow regime. Consequently, natural processes such as shifting of major shoals could have significant impact that might lead to additional maintenance dredging requirements. Modifying the Fire Island shoals did not appear to affect flow patterns farther upstream around Anchorage during flood tide. However, this conclusion is based only on visual observation without any quantifying measurements.

$f$. The smooth boundaries of the physical model might impede development of fully turbulent flow, and thus represent a scale effect. However, addition of surface roughness did not have any impact on the overall flow conditions observed in the 3-D model. This may be the result of the water passing through a perforated screen before flowing across the table, making the flow somewhat turbulent initially.

g. Although 3-D flow structures were evident throughout the flow regime, the turbulent vertical velocities did not seem as important as the horizontal motion of the gyres. Therefore, 2-D, depth-averaged numerical models might provide reasonable approximation of the overall flow in Cook Inlet. However, details of motion within the gyres or flow entrainment areas are best investigated using a physical modeling approach, preferably with minimum geometric distortion. 
$h$. The terraced bathymetry of the idealized models appeared to inhibit water exchange between depth levels. Nevertheless, idealized flow table models provide useful qualitative insight and semiquantitative results at a modest cost. The key points are to be aware of model limitations and to assure that observed flows in the model resemble those observed in nature.

$i$. Tidal flow leaving the harbor across a vertical transition is redirected seaward closer to shore where the transition step is highest. Flow leaving the harbor over a sloping transition remained straight and mostly uniform. Neither transition should cause an increase of suspended sediment deposition potential as flow leaves the harbor region.

j. Tidal flow entering the harbor across a vertical transition creates a turbulent wake region that has suspended sediment deposition potential in the immediate area downstream of the step. In addition, fluid is advected laterally along the vertical cut which would move sediment shoreward toward the mooring area. Replacing the vertical transition with a sloping transition significantly reduced the turbulent wake resulting in smoother flow patterns and less likelihood of sediment deposition.

The value of small-scale, 3-D physical models was amply demonstrated by the Alaska District engineers who used the model to understand more fully some of the probable reasons for sedimentation at the Port of Anchorage, and to develop cost-saving dredging procedures at the port. Being able to visualize the flow with dyes and tracers provided insight and instant feedback to enhance their understanding about the primary flow processes.

\section{Turbulence scale effects study conclusions}

a. Geometric distortion of the idealized models will have some impact on the turbulent flow structures, although this impact is difficult to quantify. In general, any vertically-directed turbulent velocities will be greater in the model than in the prototype.

$b$. Evaluation of potential turbulent scale effects in proposed distorted physical models requires good understanding of dominant flow patterns in the prototype along with knowledge of boundaries where flow separation and/or turbulence generation might occur. Evaluation must be site-specific.

c. Theoretical analysis showed that flow around bends in the absence of flow separation is reasonably well simulated in a distorted model, and the physical model includes more physical reality than depth-averaged numerical models. However, bottom roughness may play a critical role in correct similitude of river bend flows.

d. Flow separation and turbulence generated at vertical edges will not have a scale effect in geometrically distorted models because vertical turbulent fluctuations are small.

e. Flow separation at vertical edges with constraining downstream jetties will be similitude in distorted models with good correspondence in resulting flow patterns in the main flow and in the entrainment region. 
f. Flow separation at a vertical step where the turbulence is manifested primarily in the vertical plane will not have any significant scale effect in geometrically distorted models.

g. Steeper slopes in the distorted model will tend to generate less vertical fluid motion. Consequently, a scale effect will occur between a prototype where flow turbulence is generated at a sloping boundary. However, the scale effect is strongest near the bottom and appears to lessen closer to the free surface. Also, the scale effect seemed to be restricted to the immediate vicinity of the jet boundary; however, potential impacts farther downstream were not evaluated due to the limited measurement region of the experiments.

Importance of turbulent scale effects in a geometrically distorted physical model relates directly to the processes being studied, and whether or not flow turbulence is a dominant forcing of that problem. Specifically, the Alaska District is considering construction of a large physical model of Cook Inlet with a geometric distortion of 4 (horizontal/vertical length scale), and the Alaska District engineers are concerned about potential turbulence scale effects because flow separation and gyre formation are known to be important influences at Cook Inlet.

Based on (a) performance of the 3-D flow table model with horizontal-tovertical distortion of 15 , (b) theoretical analyses of potential scale effects, and (c) flow table experiments, it is the opinion of the report authors that turbulent scale effects in the proposed distorted physical model of Cook Inlet would not significantly influence model results. In other words, hydrodynamic flow patterns, regions of flow separation, generation of large-scale gyres, and results of sediment tracer and dye injection experiments from the distorted model would closely resemble those of an undistorted model. Differences will occur in the immediate vicinity of flow separation boundaries closer to the bottom, but these differences are expected to be localized and should not influence overall flow patterns. Three-dimensional flow structures will also have a scale effect with vertical velocity components being stronger than they should be. However, this is partially offset by steepening of slopes in the distorted model that will decrease the vertical turbulent fluctuations. An important consideration in large distorted physical models is providing sufficient bottom and boundary roughness to assure a fully turbulent boundary layer.

Geometrically distorted physical models retain all of the nonlinearities inherent in complex flow situations, including turbulence generation. Even though known scale effects will alter the value of some turbulence terms, the overall turbulence flow field will still exhibit most of the behavior expected from an undistorted physical model. For cases where turbulence and 3-D flow patterns dominate, the distorted physical model provides more reality than depth-averaged numerical models or even 3-D numerical flow models which discard or linearize the convective accelerations and/or simplify terms related to turbulence. Consequently, where space limitations prohibit construction of an undistorted model, geometrically distorted physical model may be the best tool for examining engineering problems related to flow turbulence and 3-D flow fields. 


\section{References}

Engelund, F. (1974). "Flow and bed topography in channel bends," Journal of the Hydraulics Division 100(HY11), American Society of Civil Engineers, $1,631-1,648$.

Hughes, S. A. (1993). Physical models and laboratory techniques in coastal engineering. Advanced Series on Ocean Engineering, Vol 7, World Scientific, Singapore.

Thorne, C. R., and Abt, S. R. (1993). "Velocity and scour prediction in river bends," Contract Report HL-93-1, U.S. Army Engineer Waterways Experiment Station, Vicksburg, MS. 


\section{Appendix A Case 1 - Flow Separation at Vertical Edge - Free Jet}

This Appendix contains the complete set of comparisons between prototype and geometrically-distorted models for the case of flow separation on the boundary of a free jet created by a gap with vertical edges. See Chapter 5 for a description of the experiments and discussion of results. 


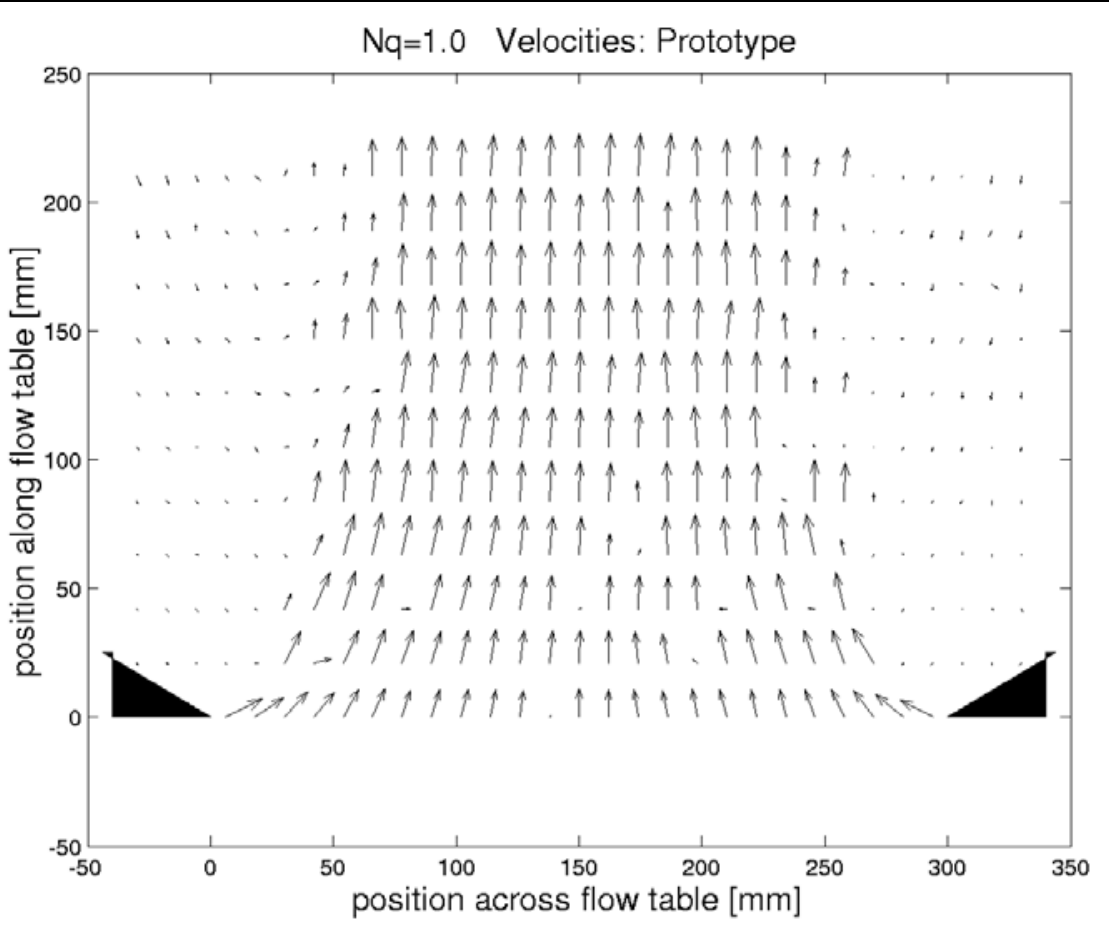

Figure A1. Case 1, $Q=1.5 \mathrm{~L} / \mathrm{sec}$, prototype

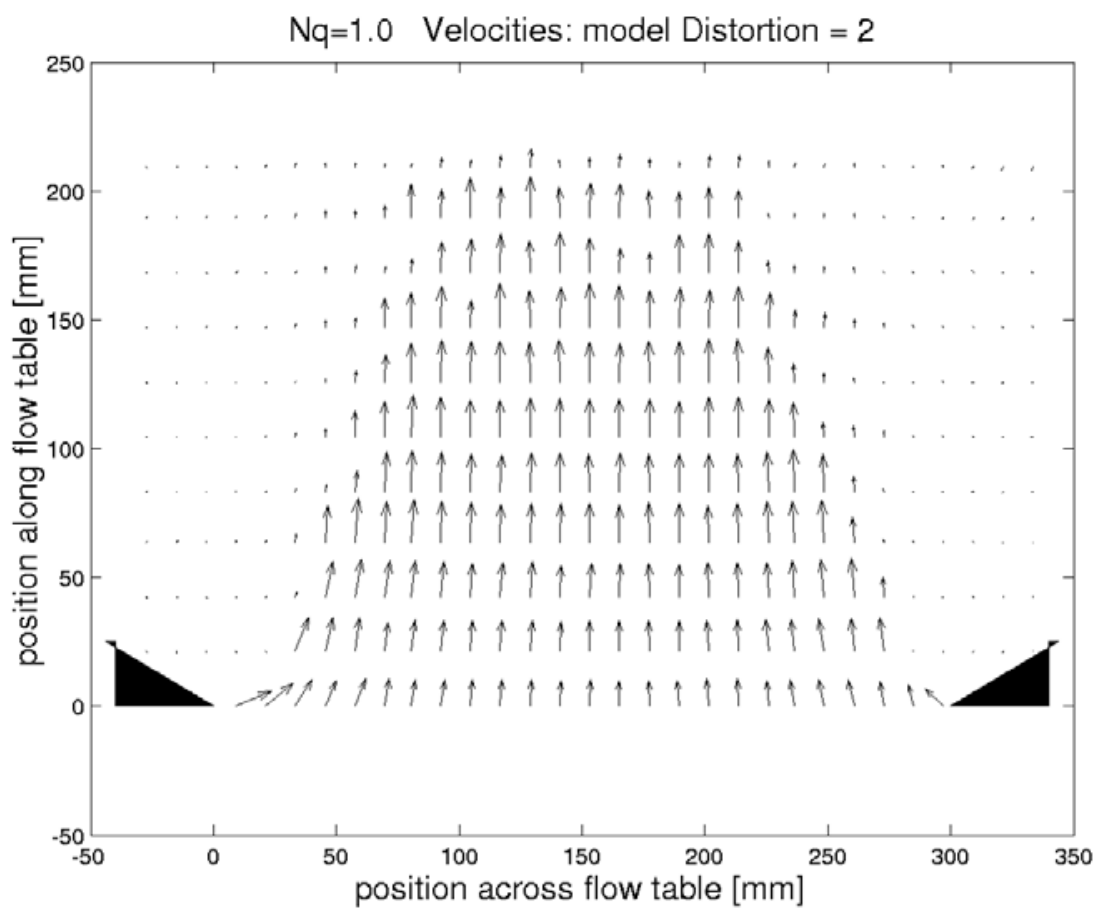

Figure A2. Case 1, $Q=1.5 \mathrm{~L} / \mathrm{sec}$, distortion $=2$ 


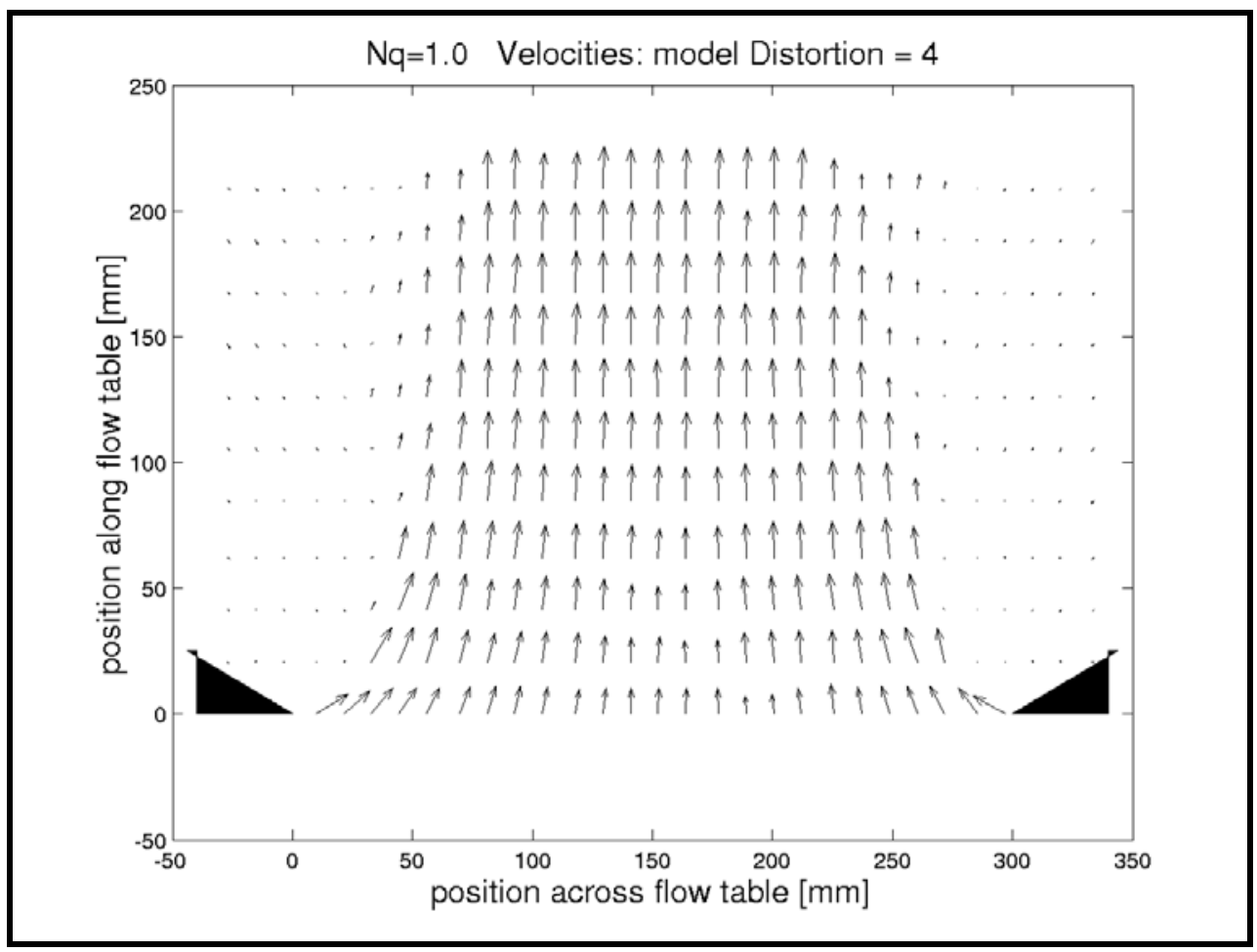

Figure A3. Case 1, $Q=1.5 \mathrm{~L} / \mathrm{sec}$, distortion $=4$

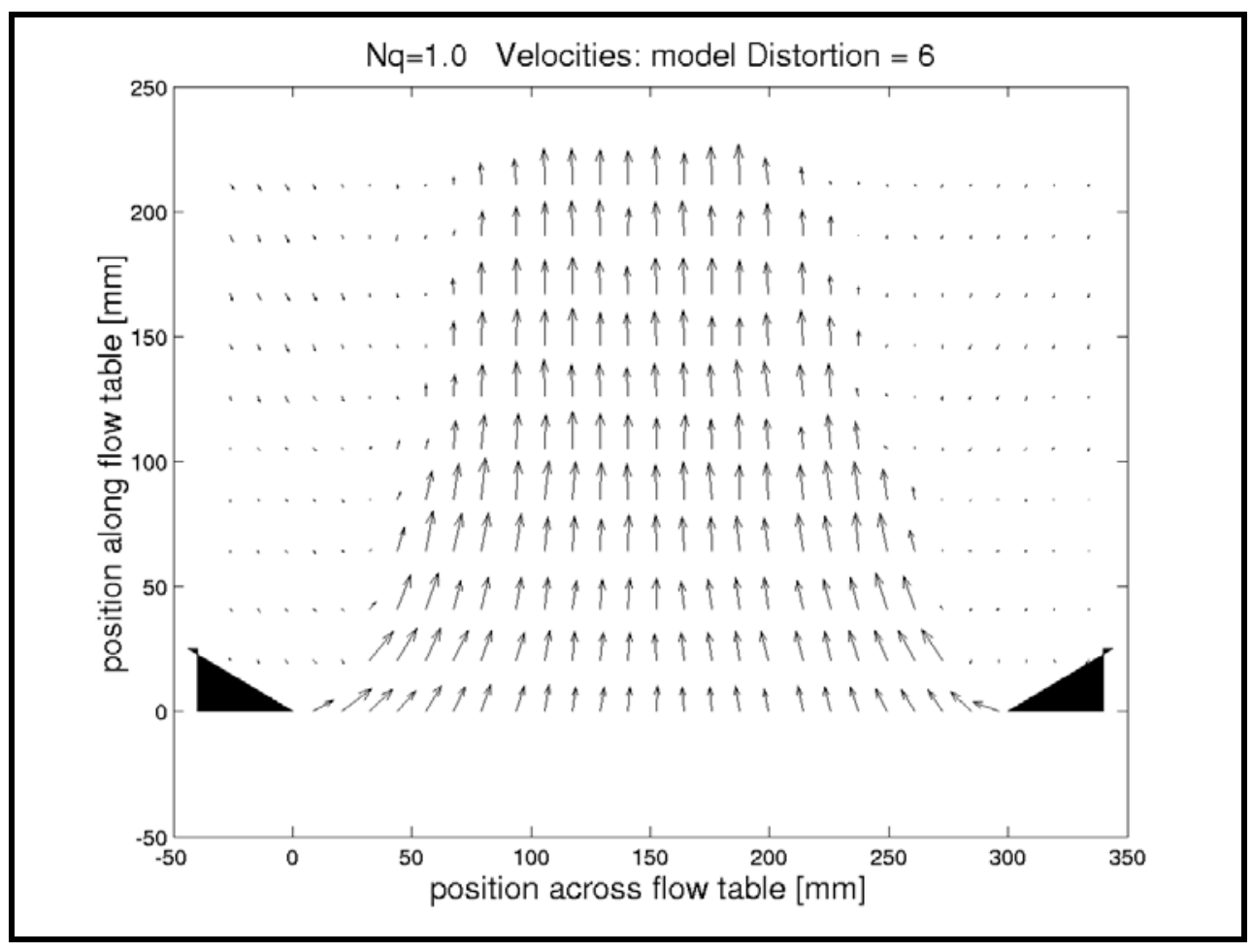

Figure A4. Case $1, Q=1.5 \mathrm{~L} / \mathrm{sec}$, distortion $=6$ 


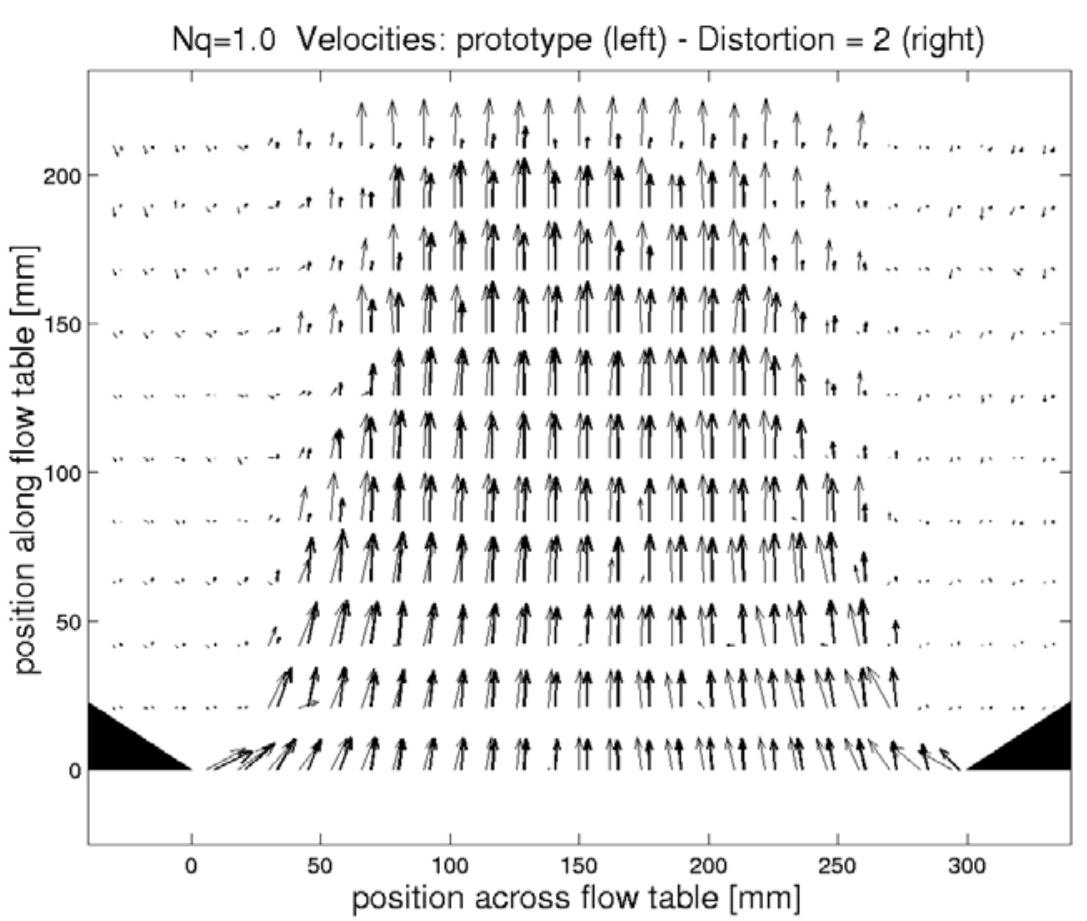

Figure A5. Case 1, $Q=1.5 \mathrm{~L} / \mathrm{sec}$, prototype vs. distortion $=2$

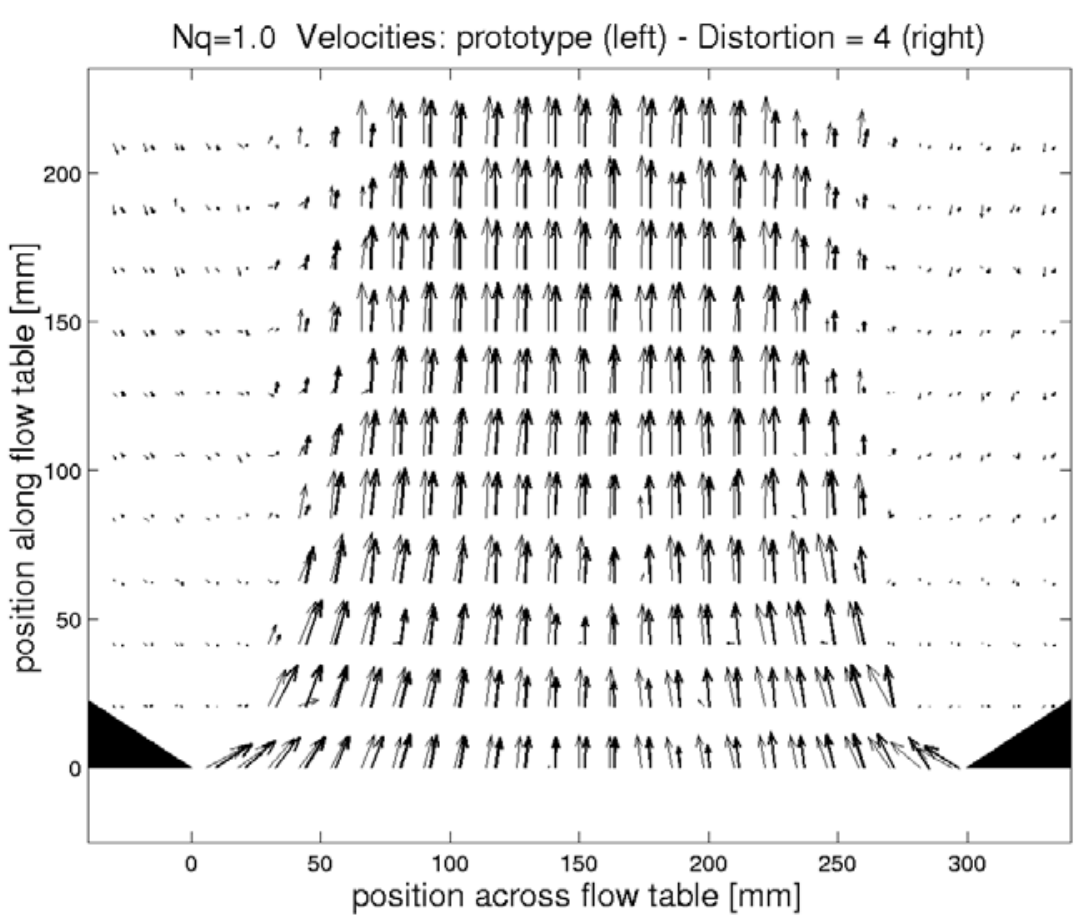

Figure A6. Case 1, $Q=1.5 \mathrm{~L} / \mathrm{sec}$, prototype vs. distortion $=4$ 


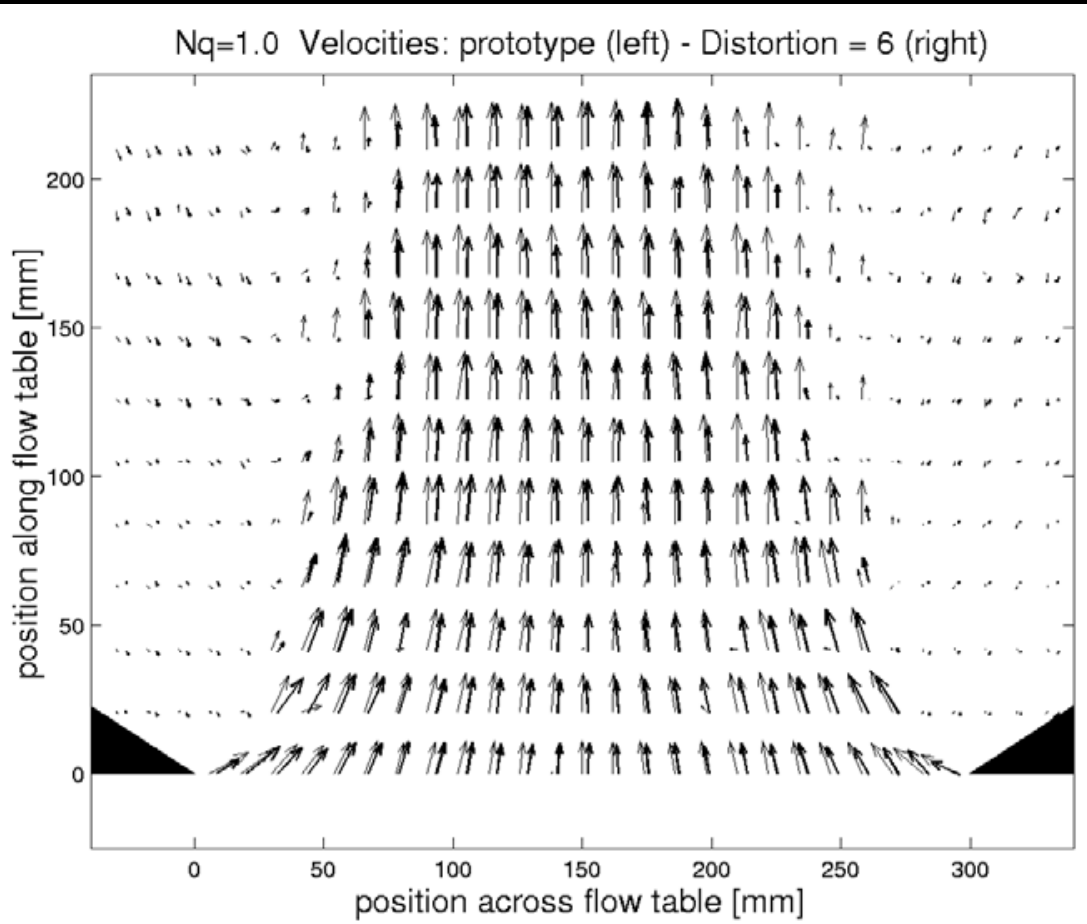

Figure A7. Case $1, Q=1.5 \mathrm{~L} / \mathrm{sec}$, prototype vs. distortion $=6$

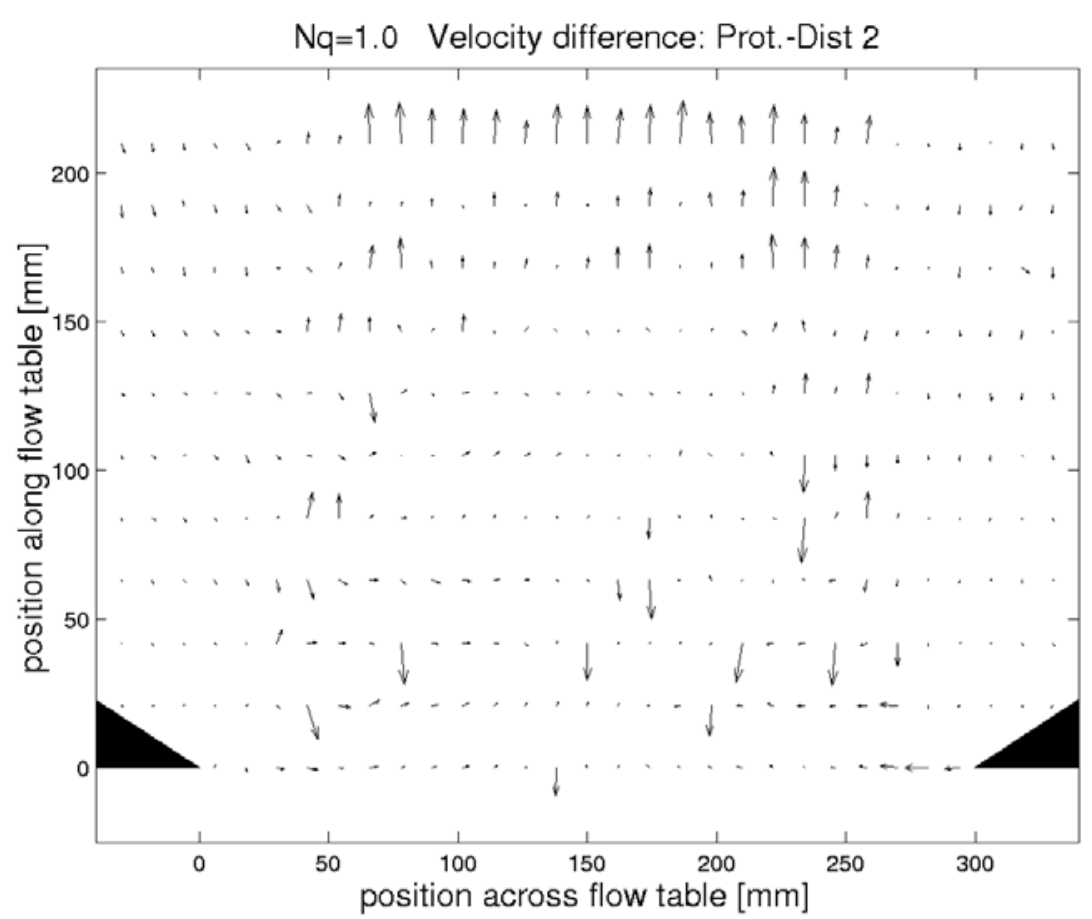

Figure A8. Case $1, Q=1.5 \mathrm{~L} / \mathrm{sec}$, prototype minus distortion $=2$ 


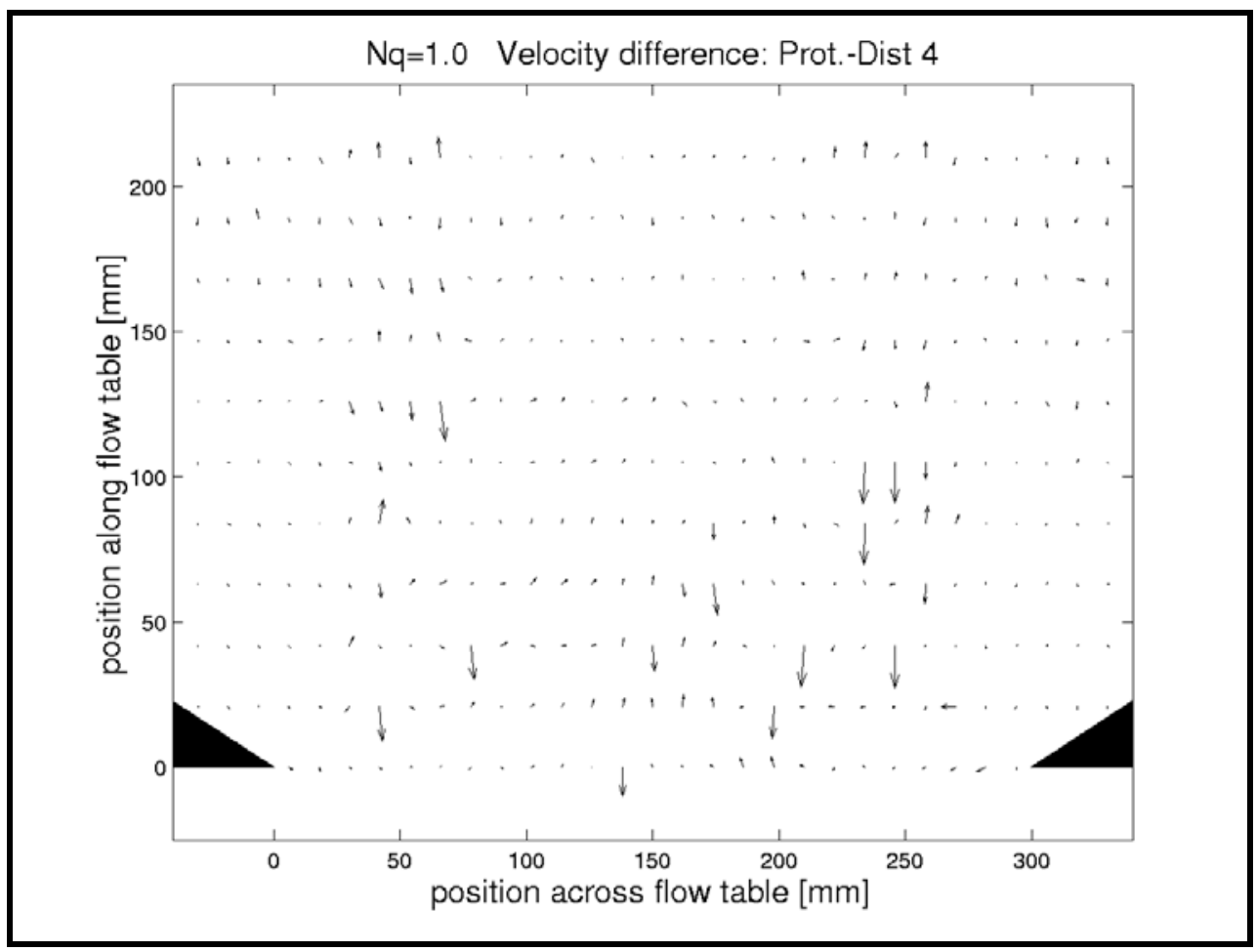

Figure A9. Case $1, Q=1.5 \mathrm{~L} / \mathrm{sec}$, prototype minus distortion $=4$

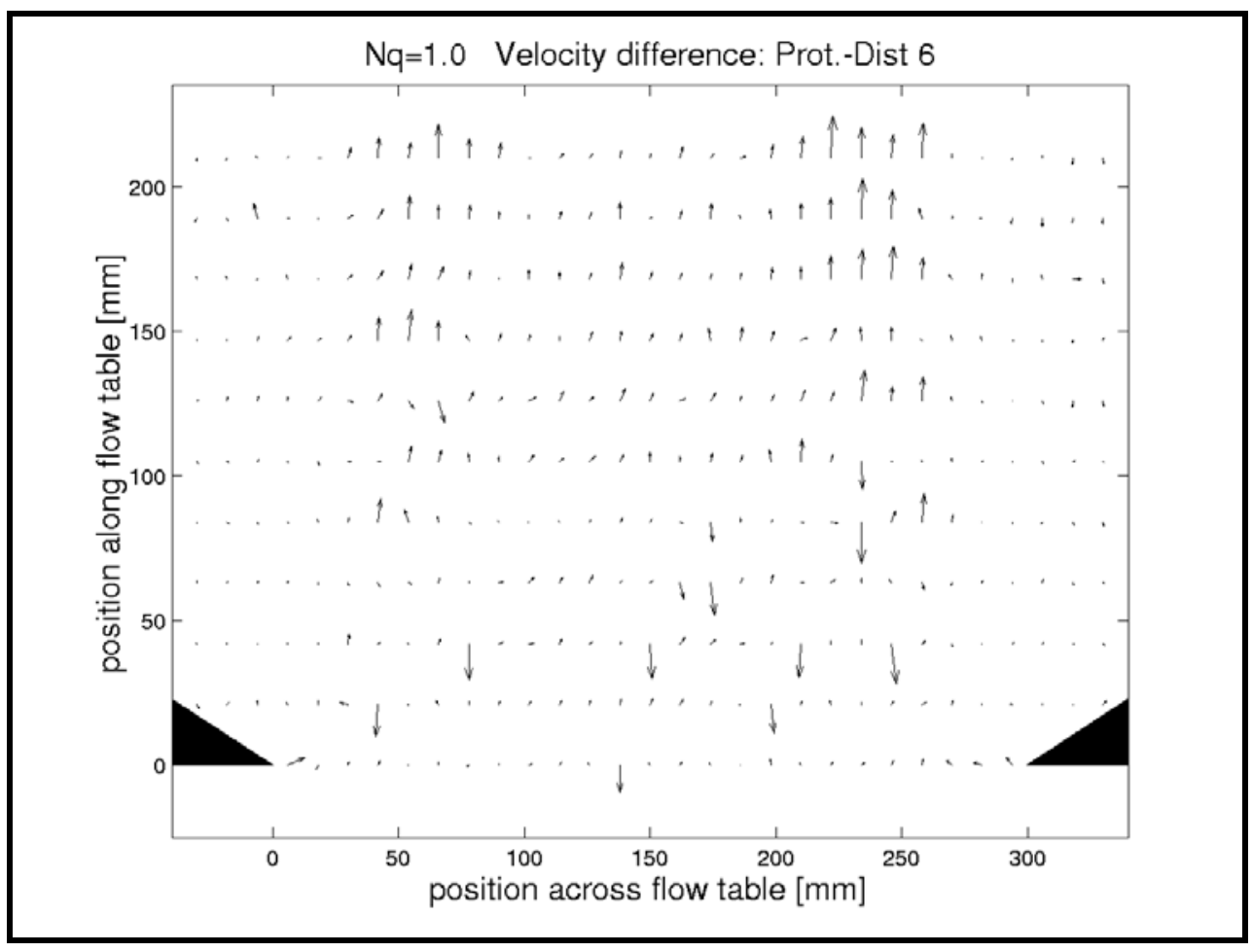

Figure A10. Case $1, Q=1.5 \mathrm{~L} / \mathrm{sec}$, prototype minus distortion $=6$ 


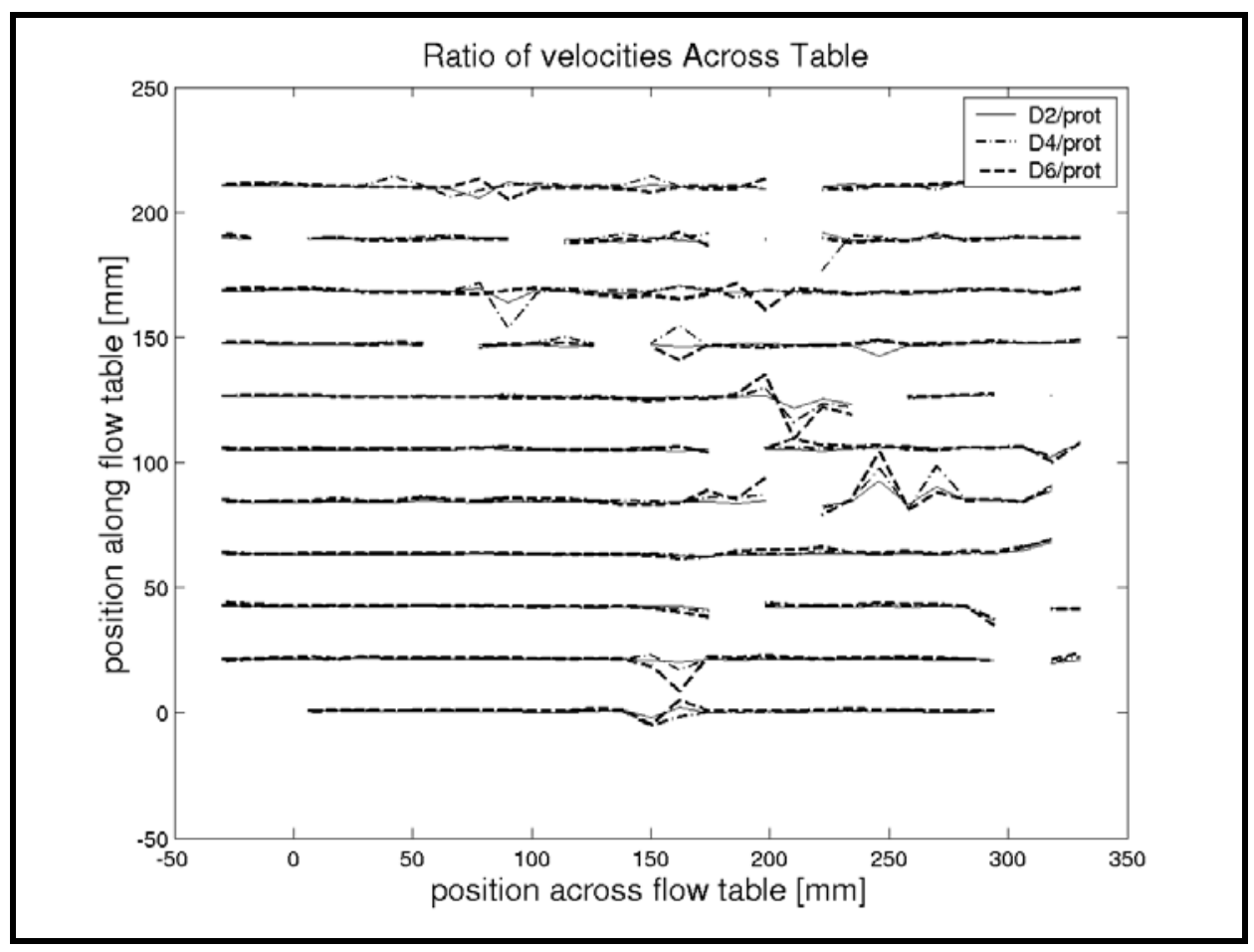

Figure A11. Case $1, Q=1.5 \mathrm{~L} / \mathrm{sec}$, ratios between prototype and distortions 2 , 4 , and 6

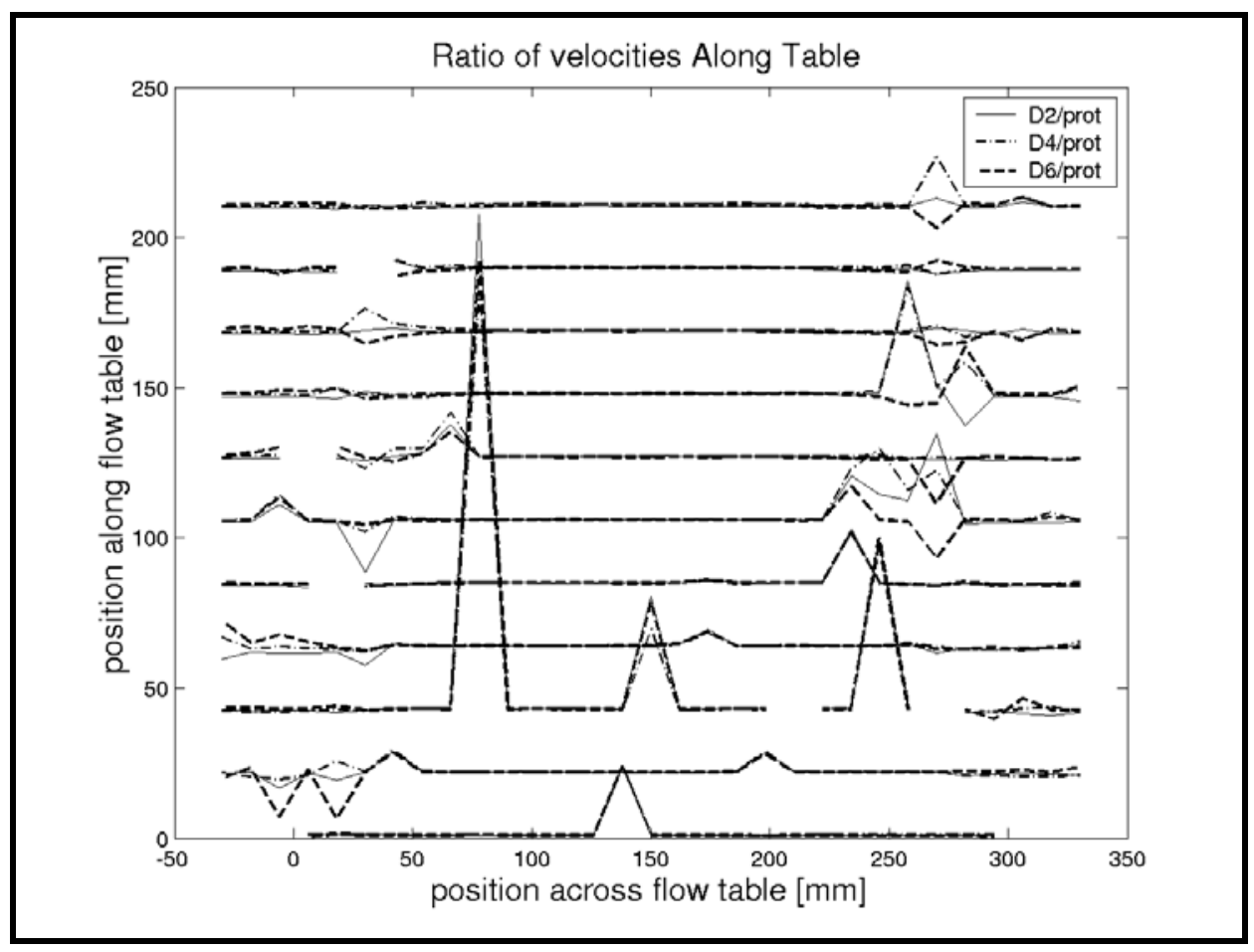

Figure A12. Case $1, Q=1.5 \mathrm{~L} / \mathrm{sec}$, ratios between prototype and distortions 2 , 4 , and 6 


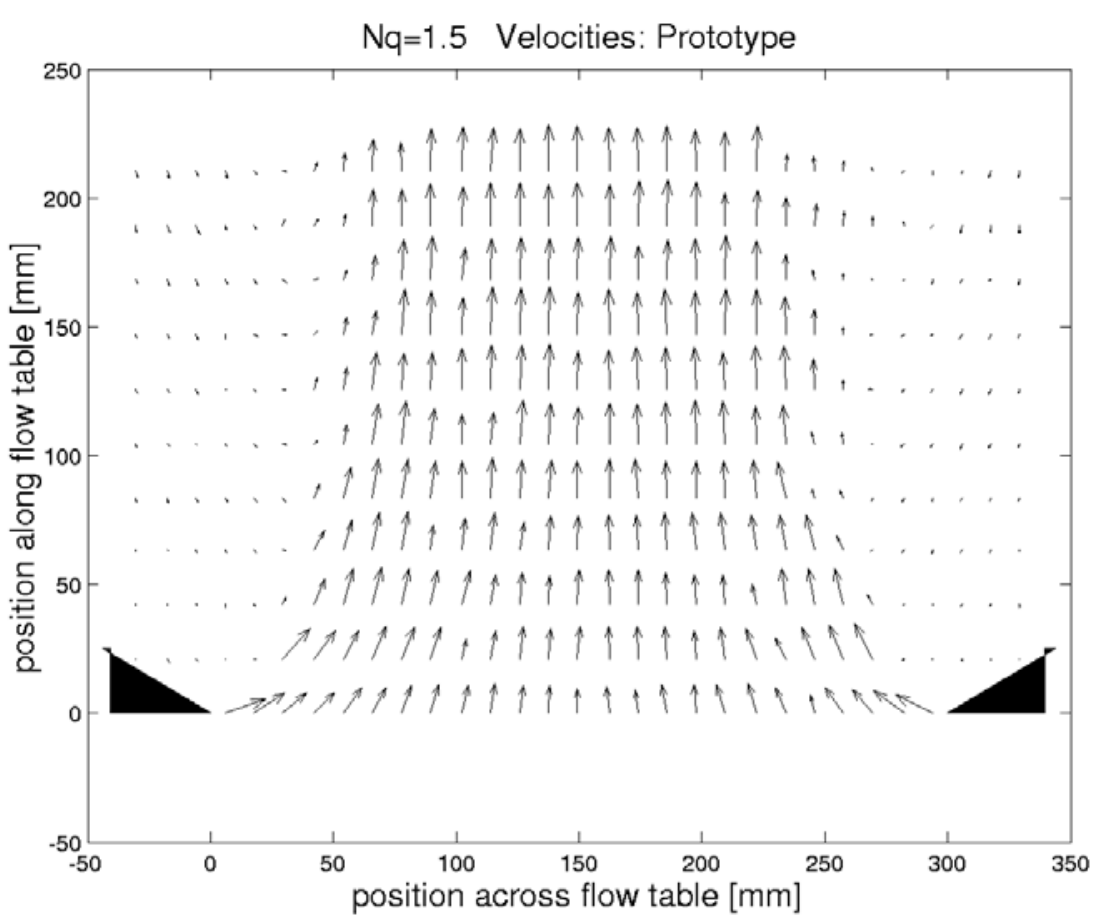

Figure A13. Case 1, $Q=1.0 \mathrm{~L} / \mathrm{sec}$, prototype

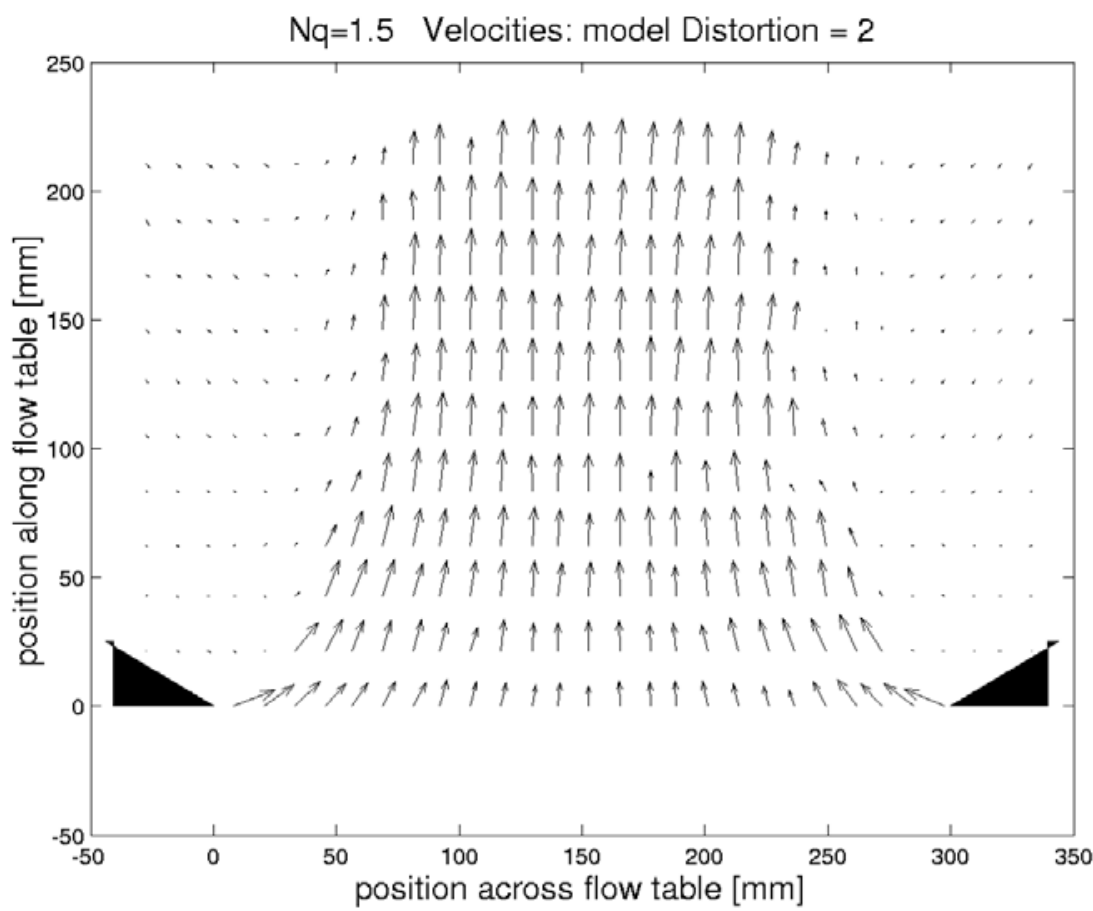

Figure A14. Case 1, $Q=1.0 \mathrm{~L} / \mathrm{sec}$, distortion $=2$ 


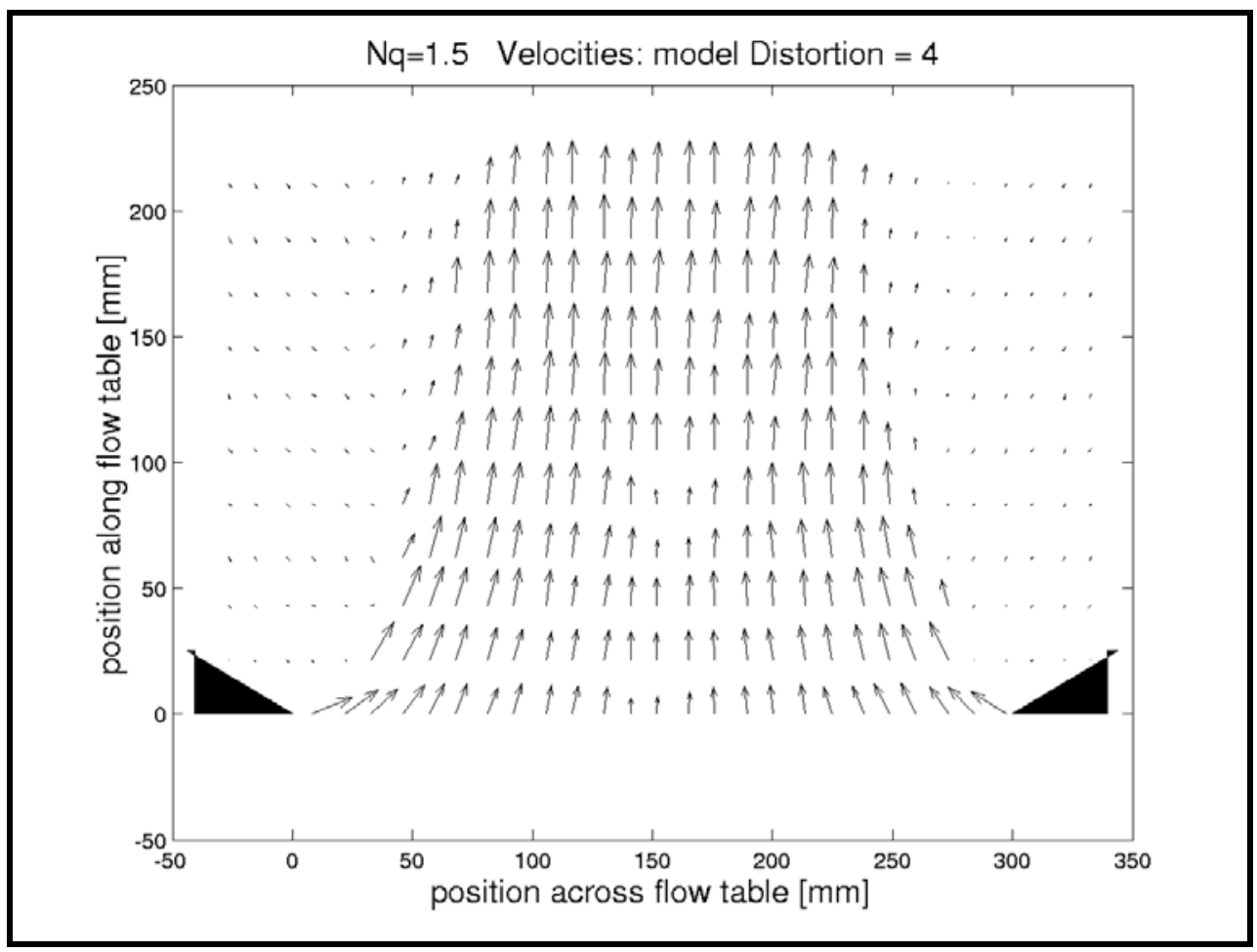

Figure A15. Case 1, $Q=1.0 \mathrm{~L} / \mathrm{sec}$, distortion $=4$

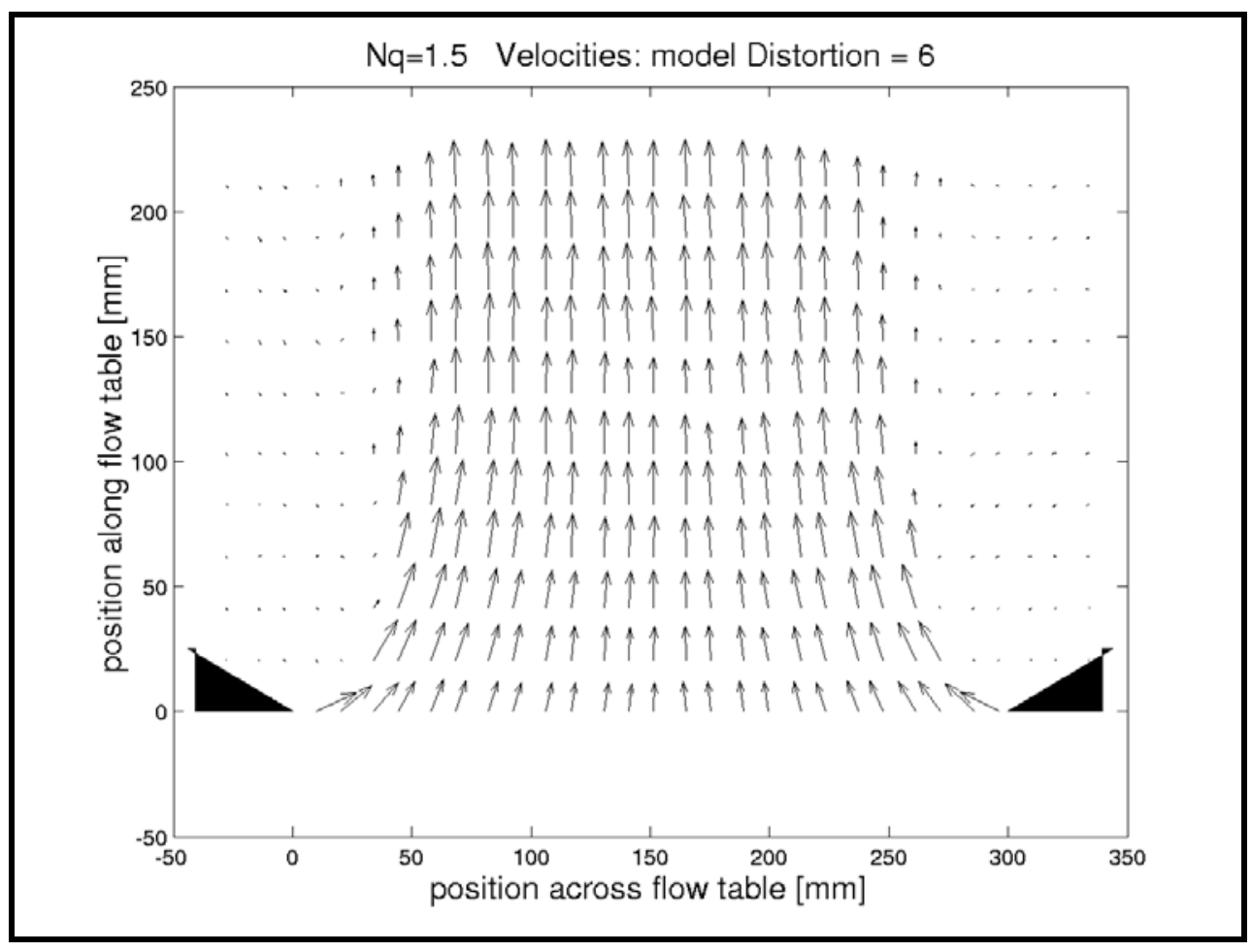

Figure A16. Case 1, $Q=1.0 \mathrm{~L} / \mathrm{sec}$, distortion $=6$ 


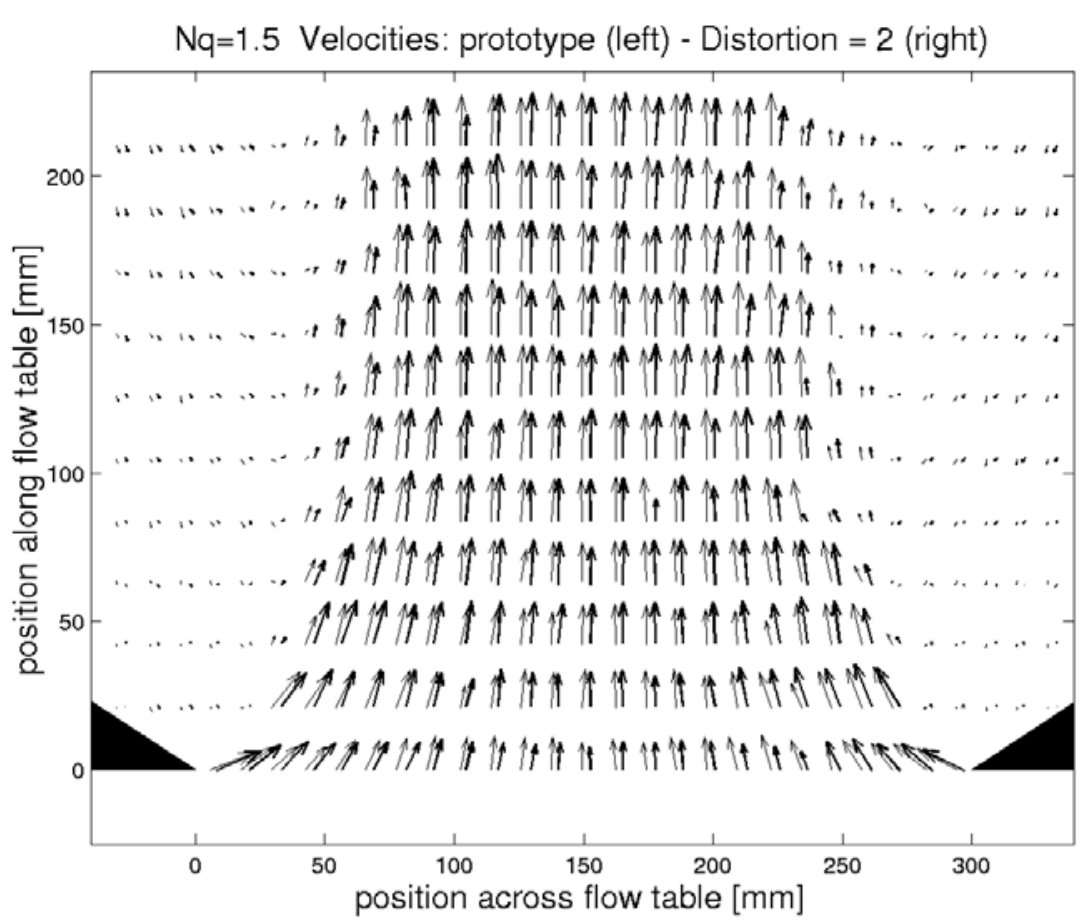

Figure A17: Case 1, $Q=1.0 \mathrm{~L} / \mathrm{sec}$, prototype vs. distortion = 2

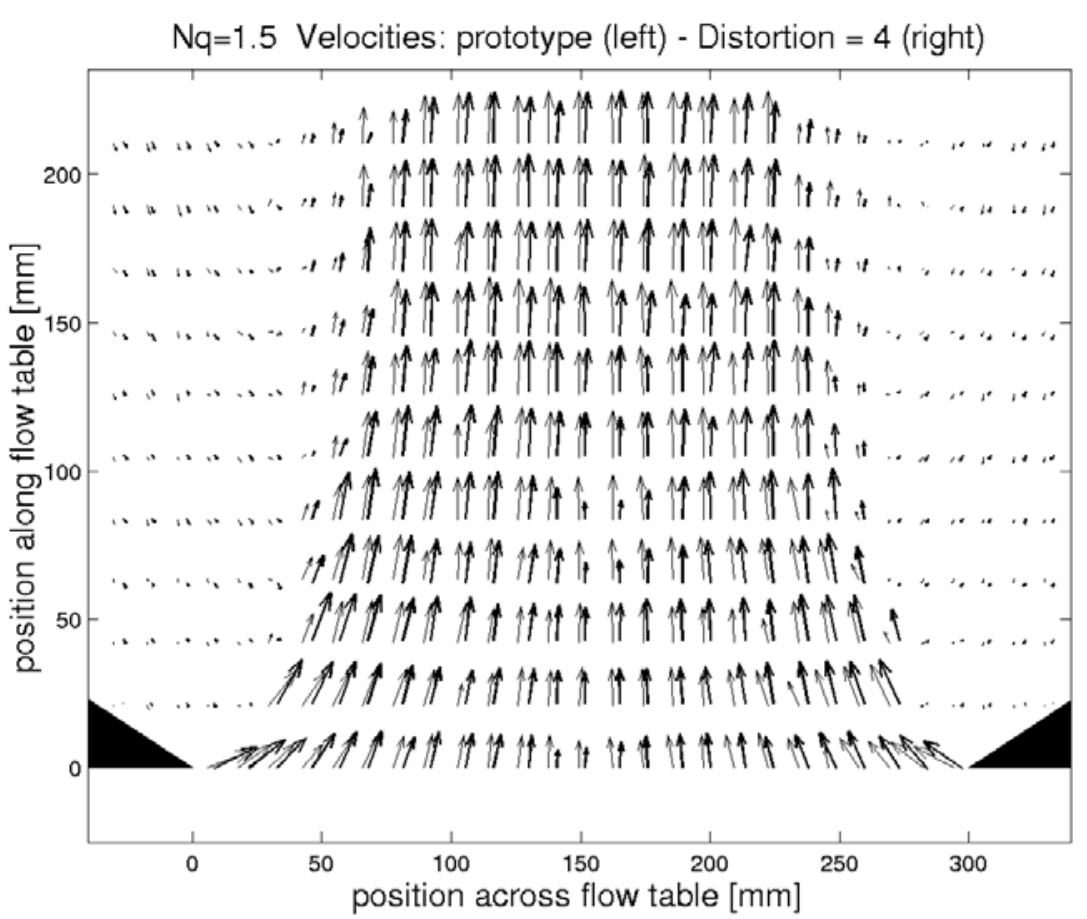

Figure A18. Case 1, $Q=1.0 \mathrm{~L} / \mathrm{sec}$, prototype vs. distortion $=4$ 


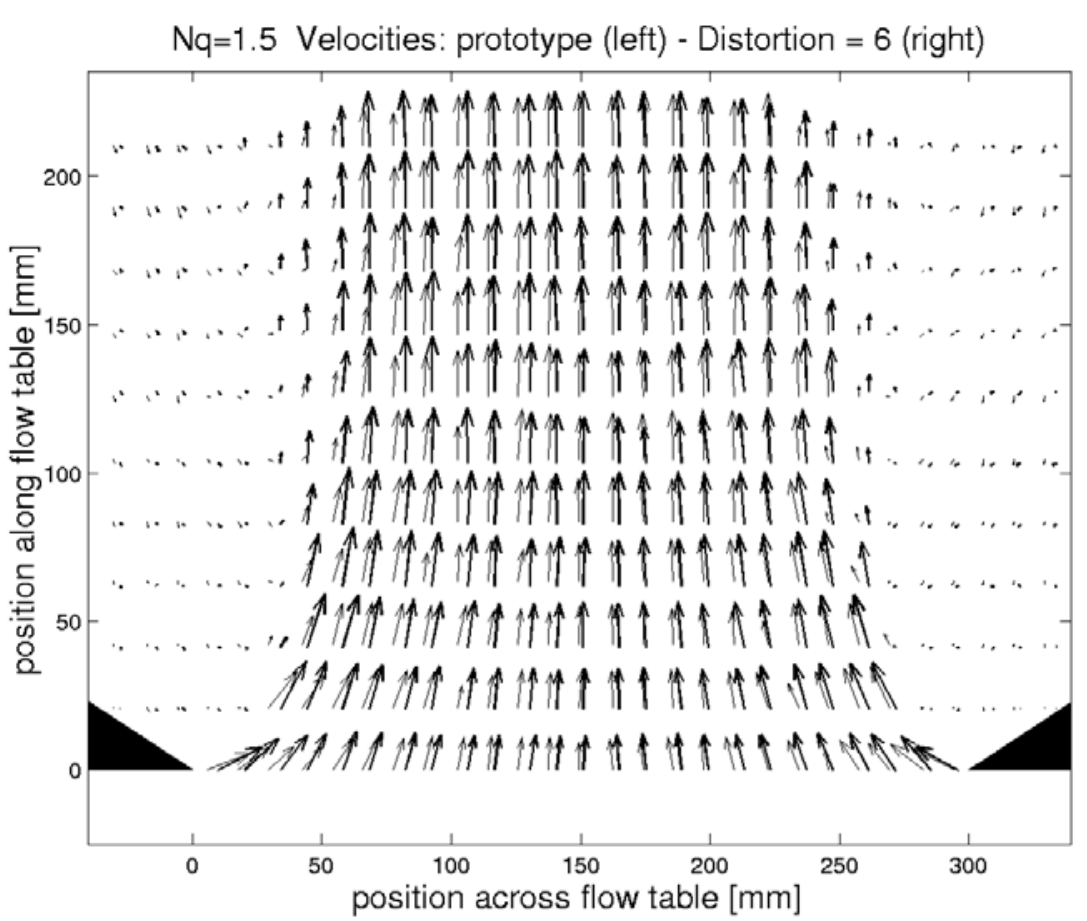

Figure A19. Case 1, $Q=1.0 \mathrm{~L} / \mathrm{sec}$, prototype vs. distortion $=6$

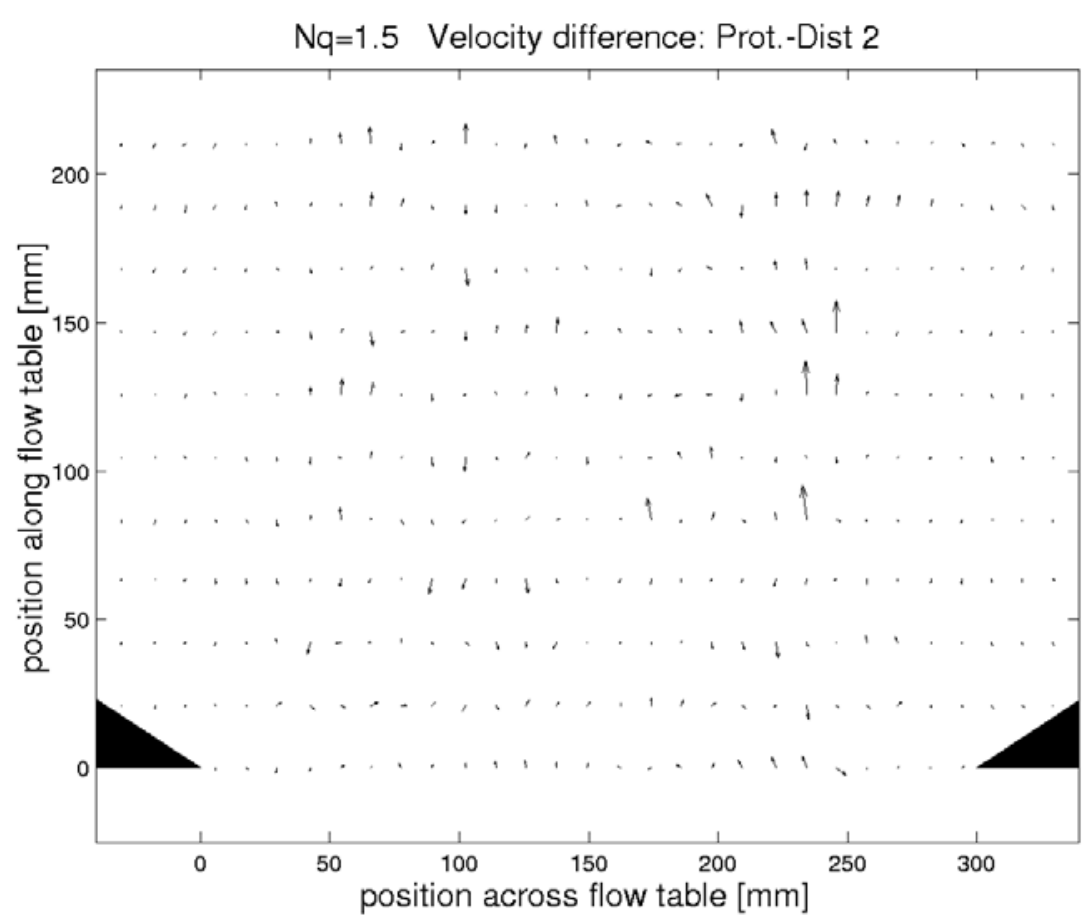

Figure A20. Case 1, $Q=1.0 \mathrm{~L} / \mathrm{sec}$, prototype minus distortion $=2$ 


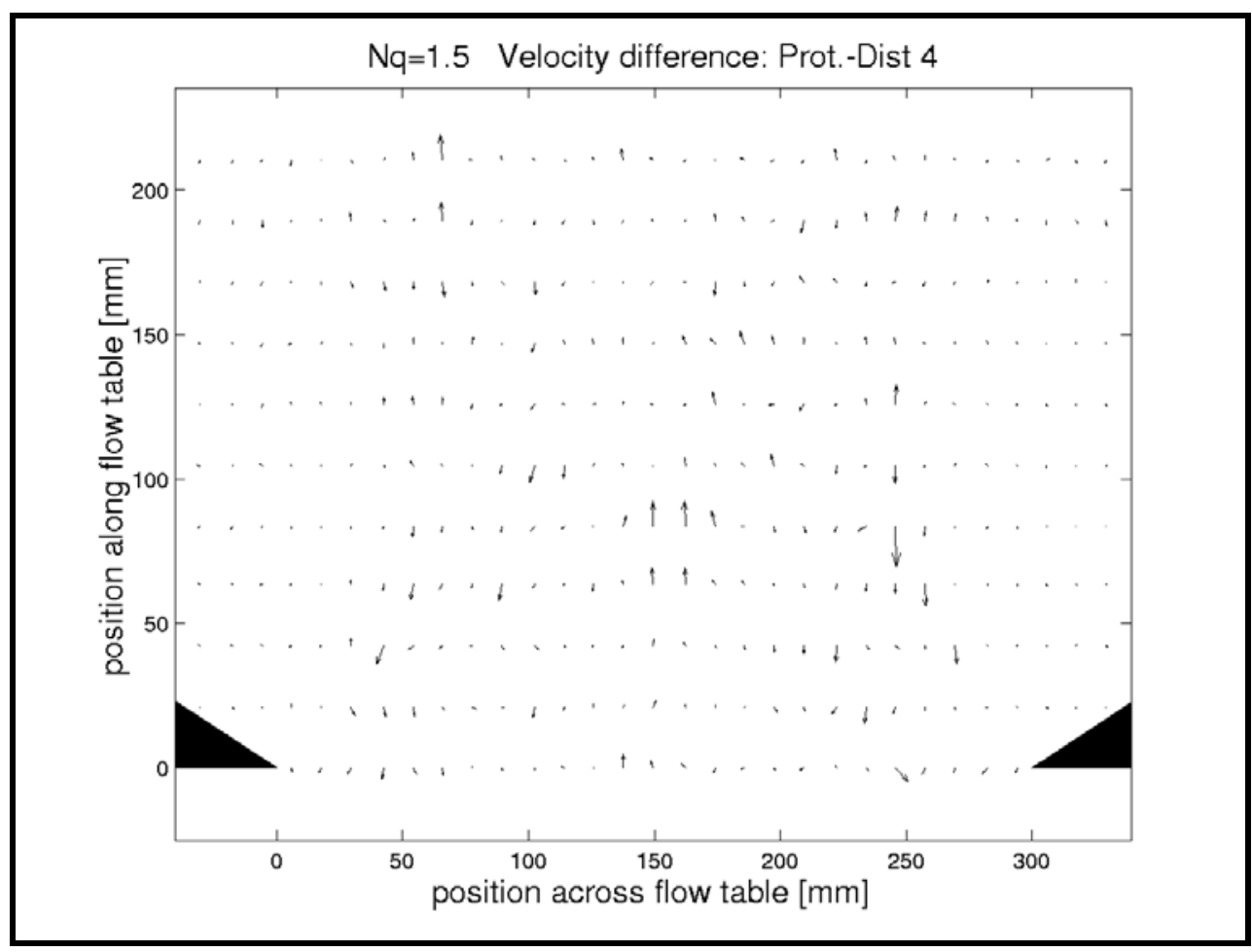

Figure $\mathrm{A} 21$. Case $1, Q=1.0 \mathrm{~L} / \mathrm{sec}$, prototype minus distortion $=4$

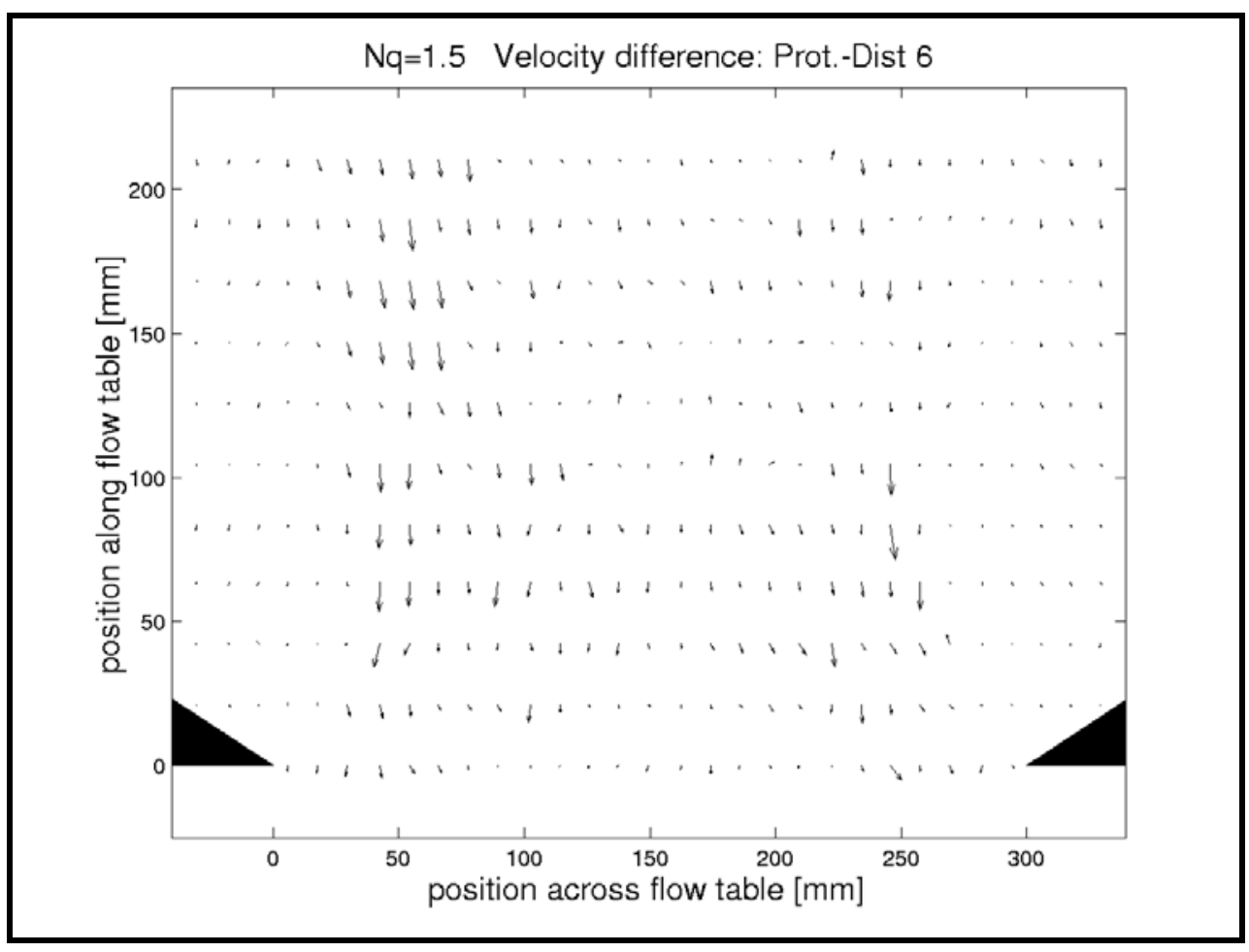

Figure A22. Case 1, $Q=1.0 \mathrm{~L} / \mathrm{sec}$, prototype minus distortion $=6$ 


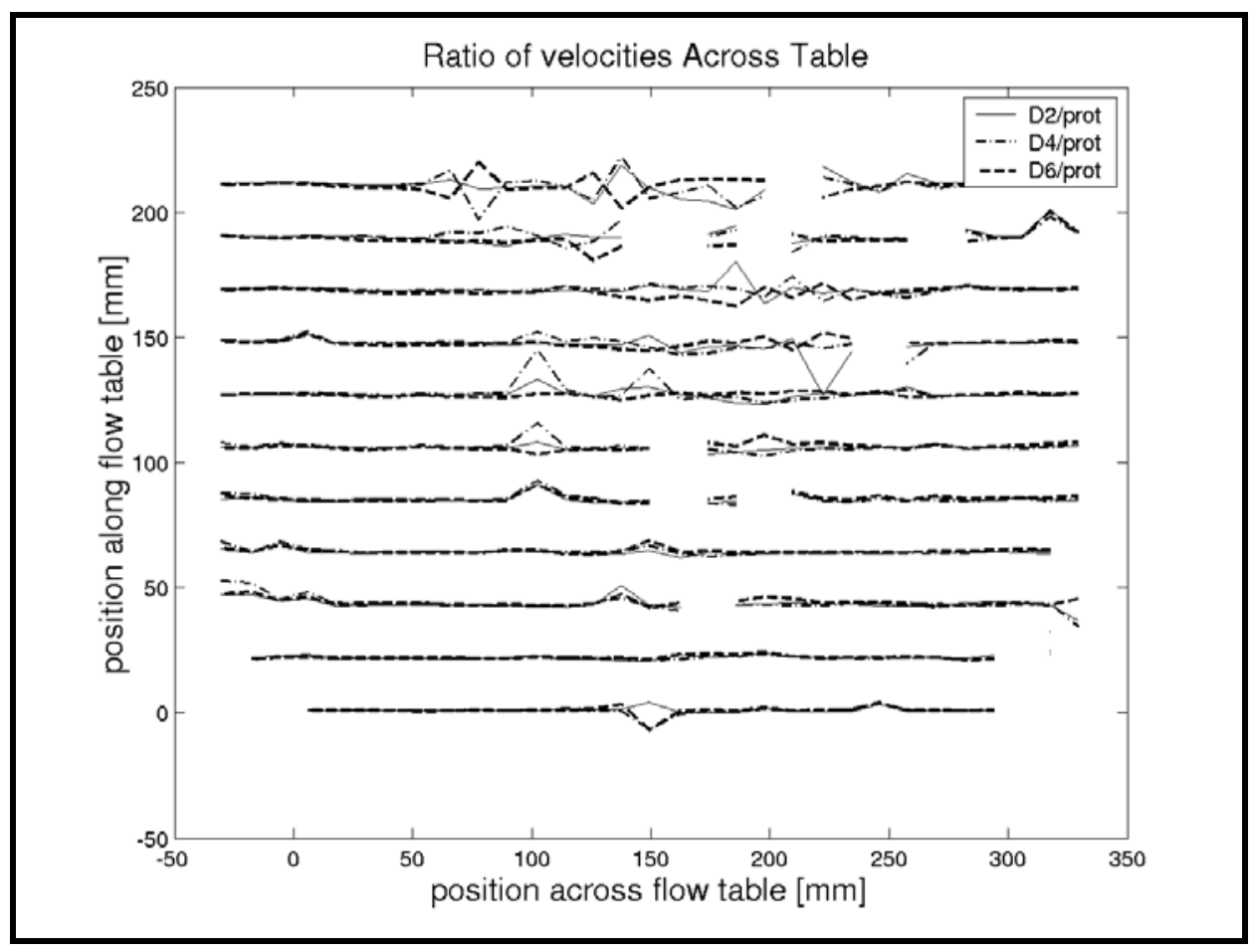

Figure A23. Case $1, Q=1.0 \mathrm{~L} / \mathrm{sec}$, ratios between prototype and distortions 2, 4 , and 6

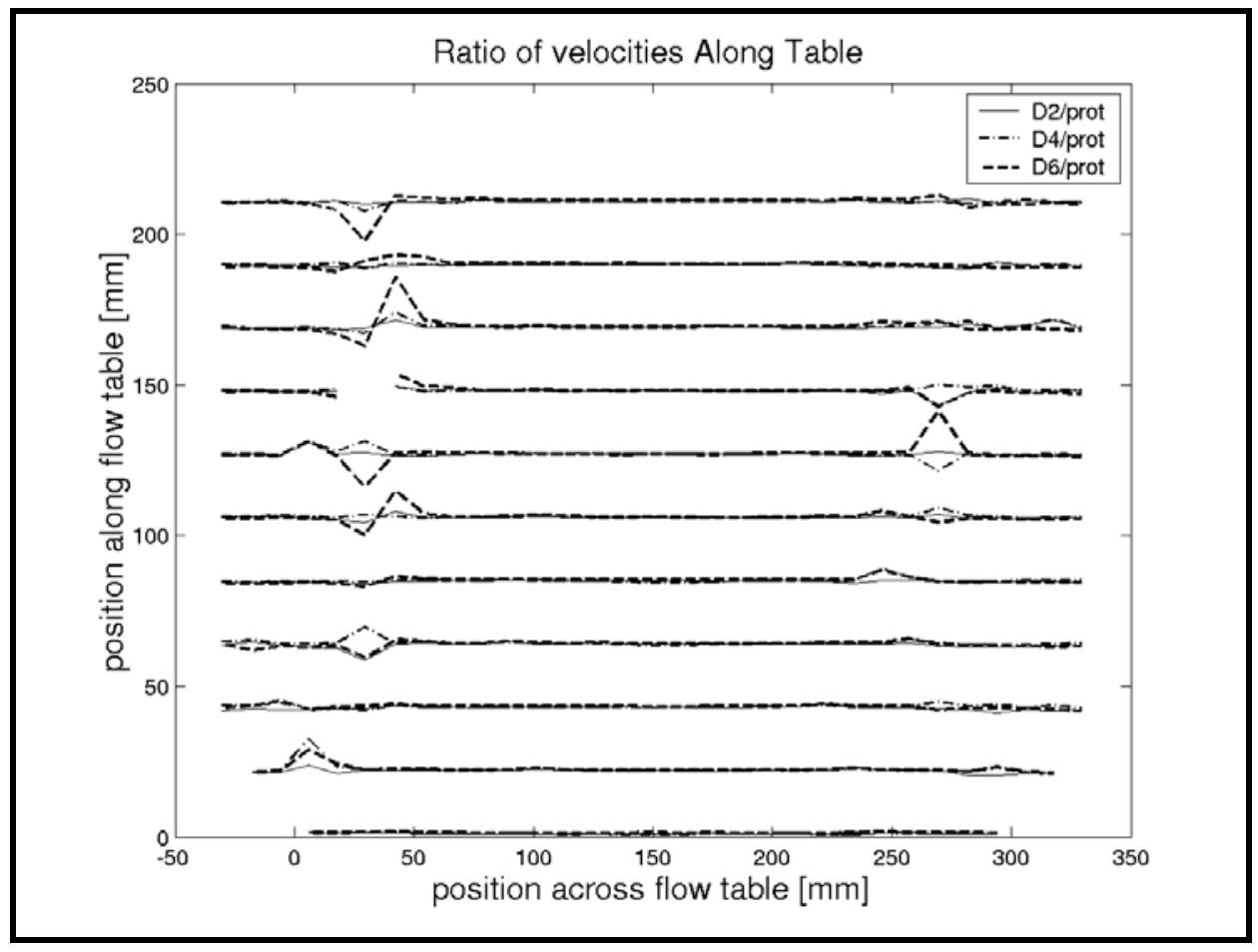

Figure A24. Case $1, Q=1.0 \mathrm{~L} / \mathrm{sec}$, ratios between prototype and distortions 2, 4 , and 6 


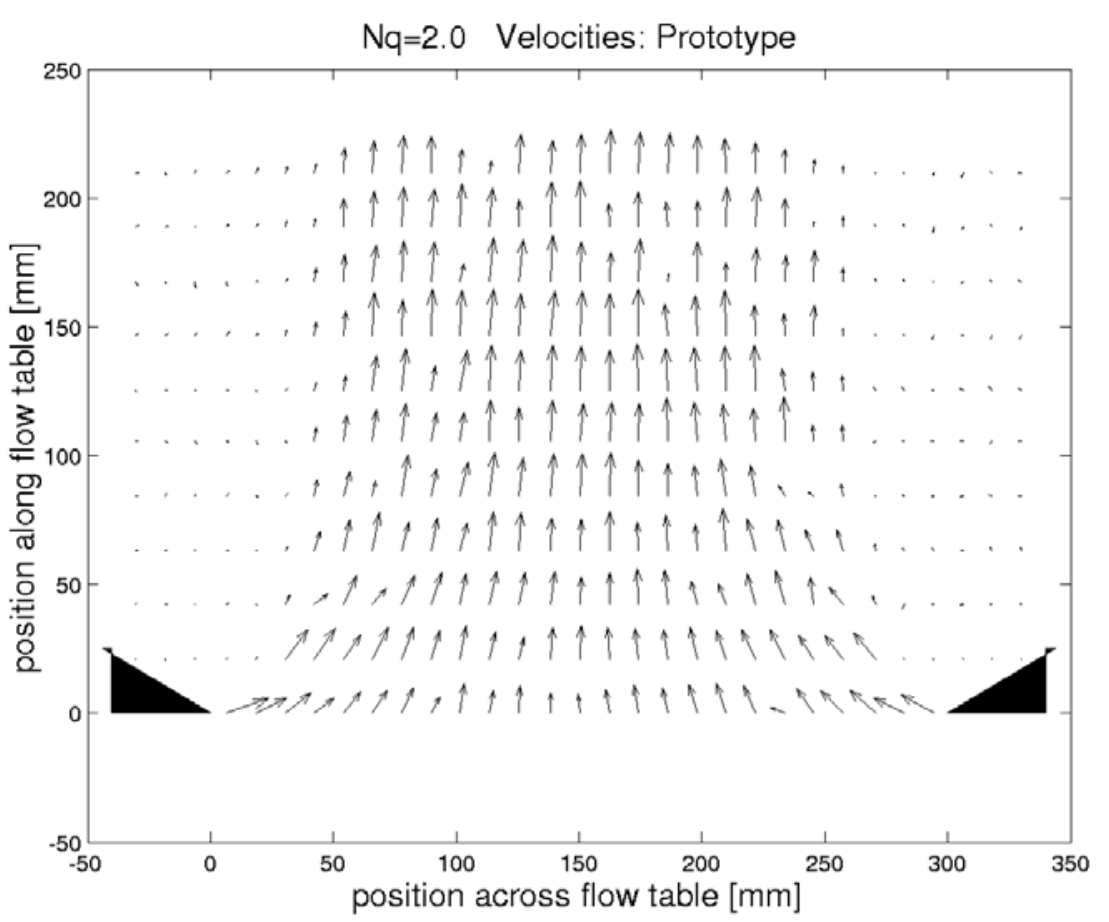

Figure A25. Case $1, Q=0.75 \mathrm{~L} / \mathrm{sec}$, prototype

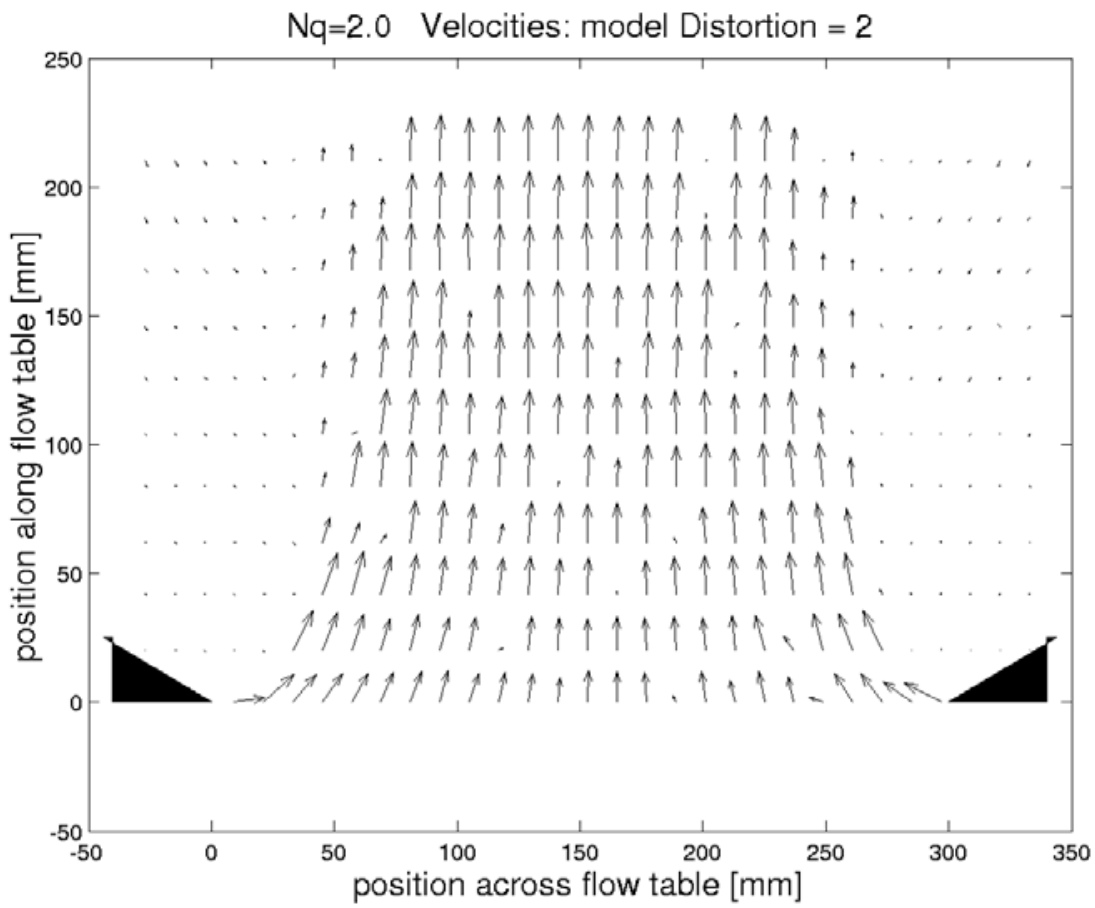

Figure A26. Case $1, Q=0.75 \mathrm{~L} / \mathrm{sec}$, distortion $=2$ 


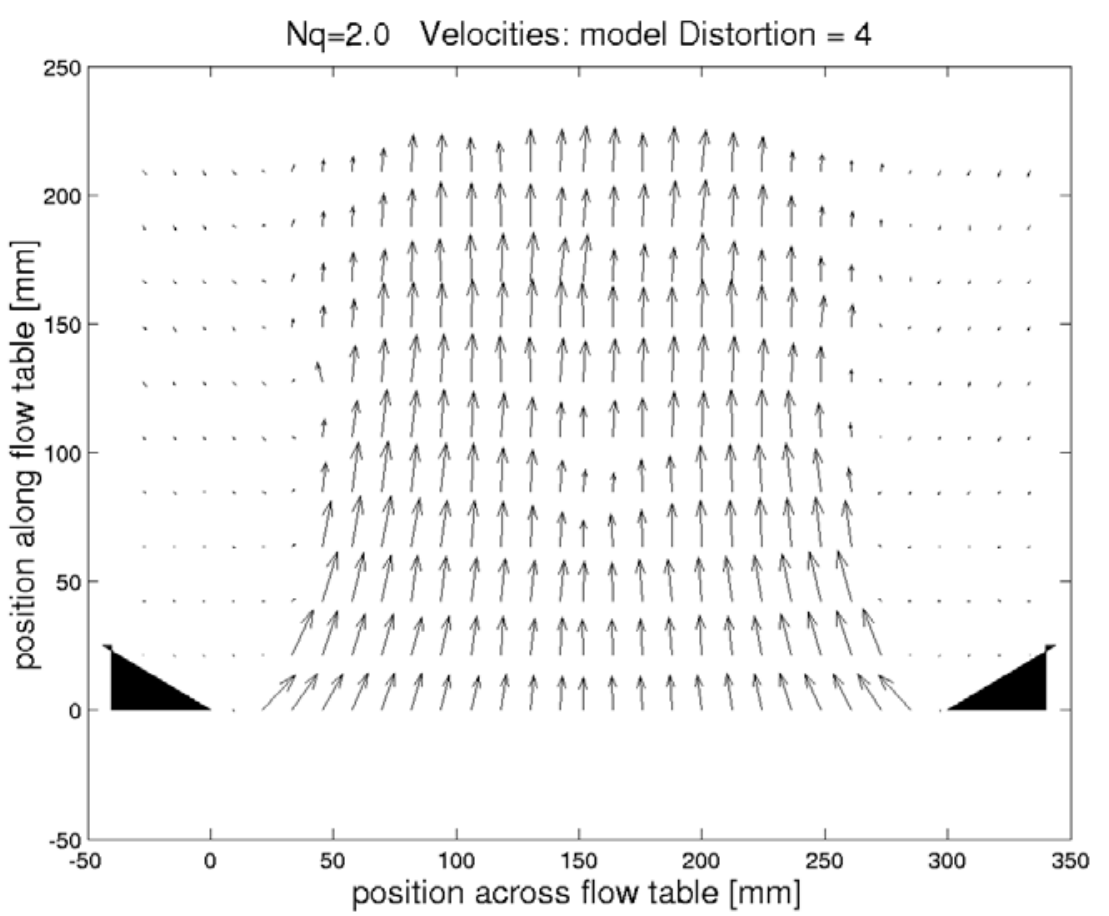

Figure A27. Case $1, Q=0.75 \mathrm{~L} / \mathrm{sec}$, distortion $=4$

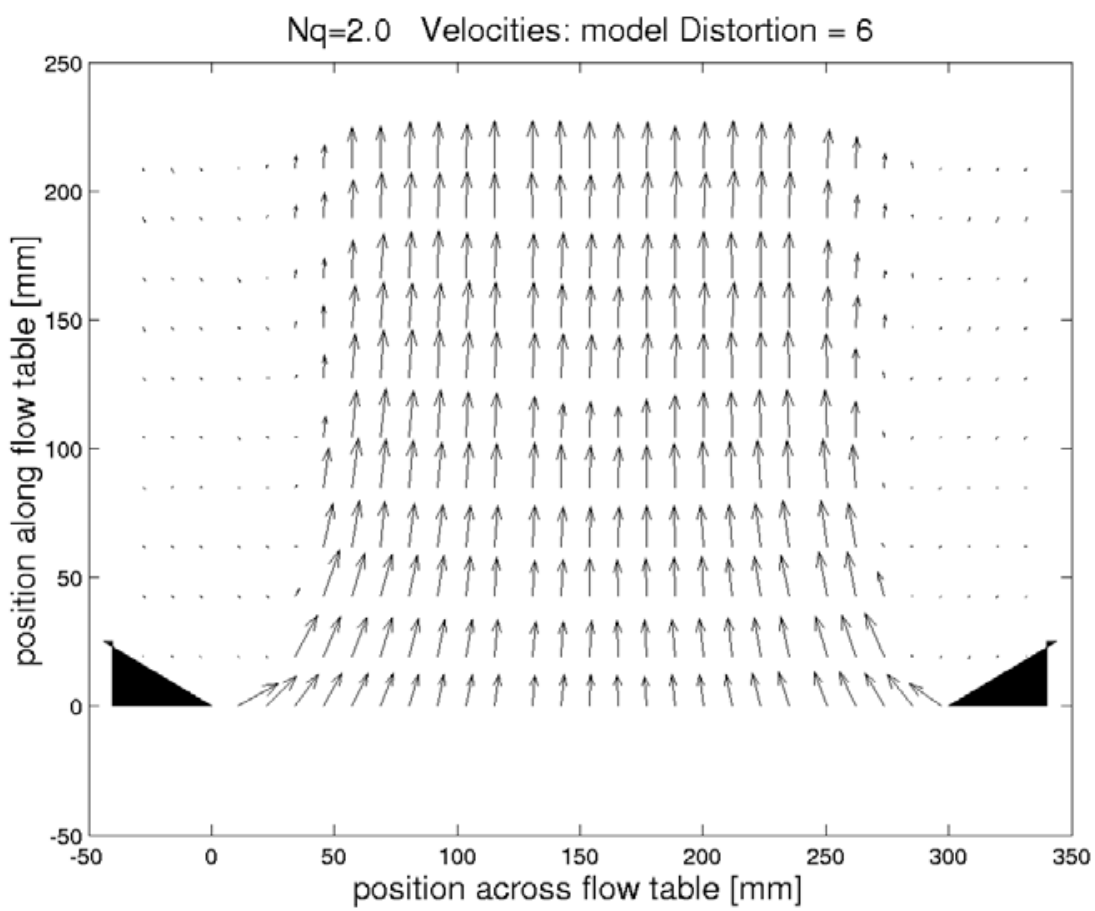

Figure A28. Case $1, Q=0.75 \mathrm{~L} / \mathrm{sec}$, distortion $=6$ 


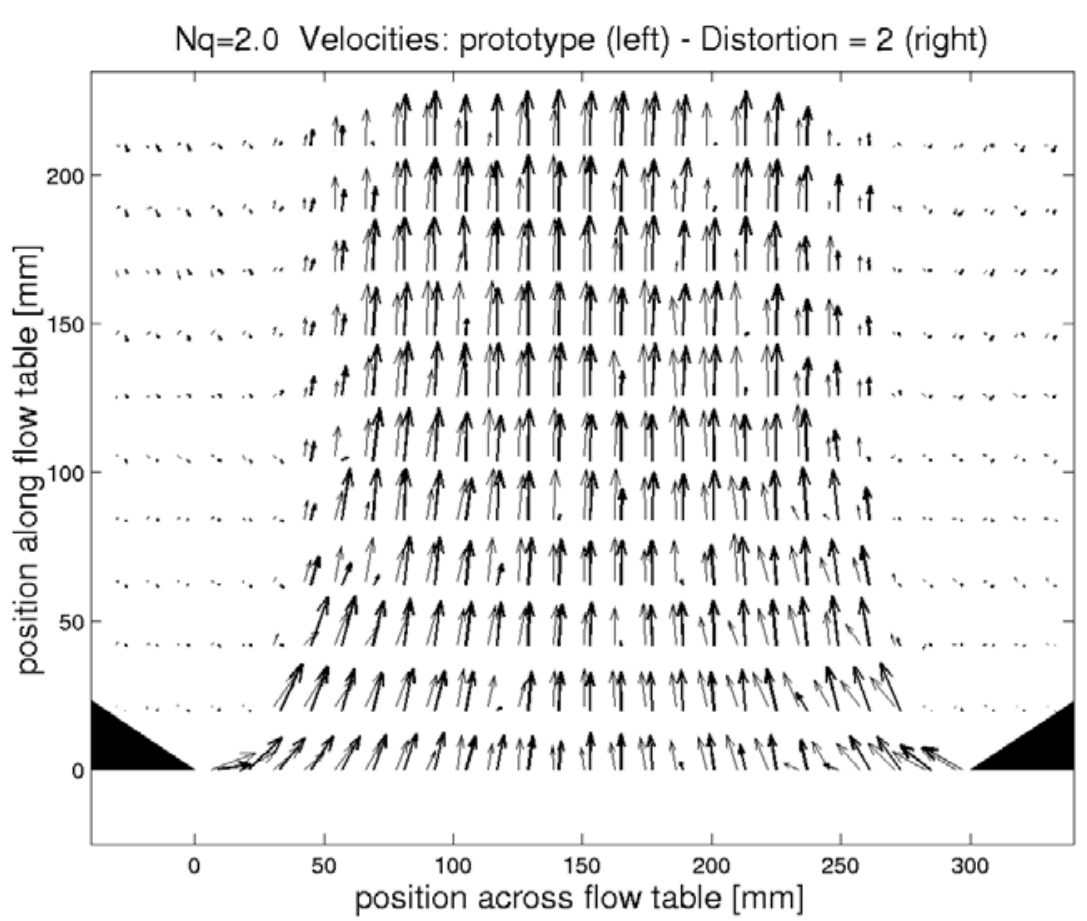

Figure A29. Case $1, Q=0.75 \mathrm{~L} / \mathrm{sec}$, prototype vs. distortion $=2$

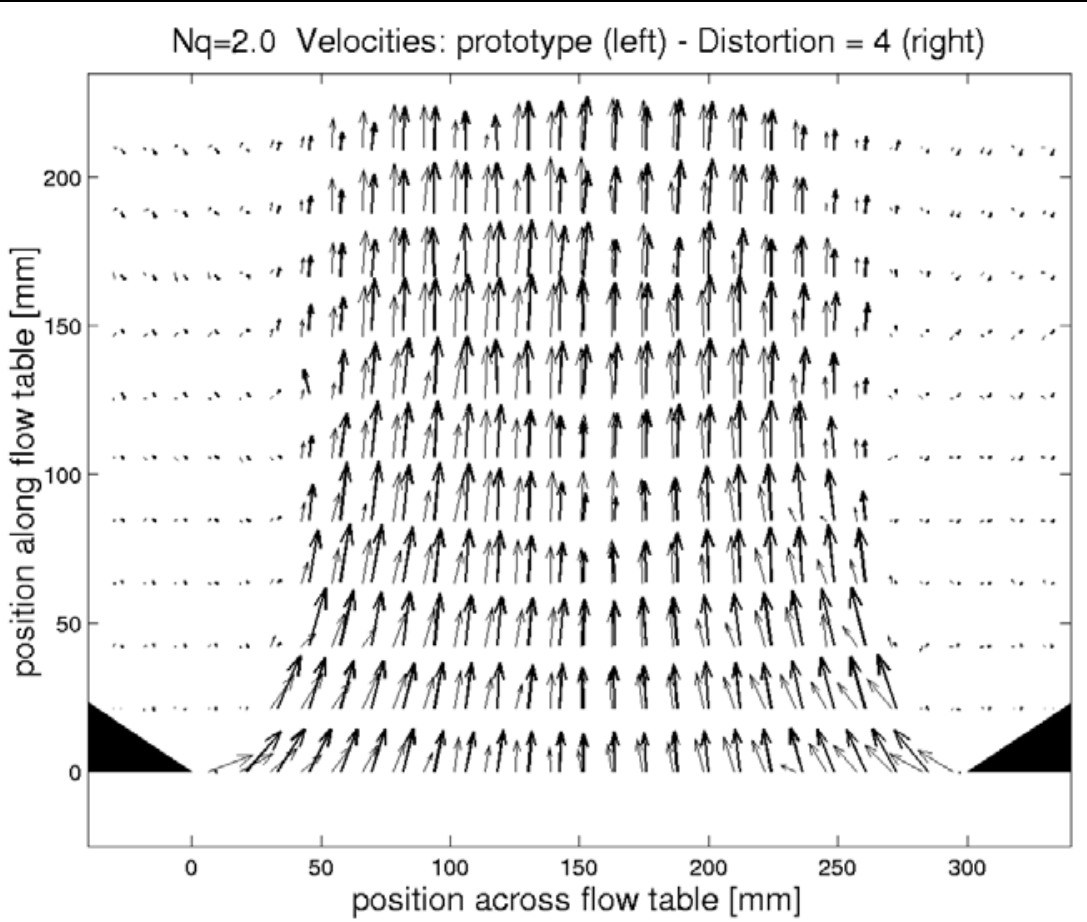

Figure $\mathrm{A} 30$. Case $1, Q=0.75 \mathrm{~L} / \mathrm{sec}$, prototype vs. distortion $=4$ 


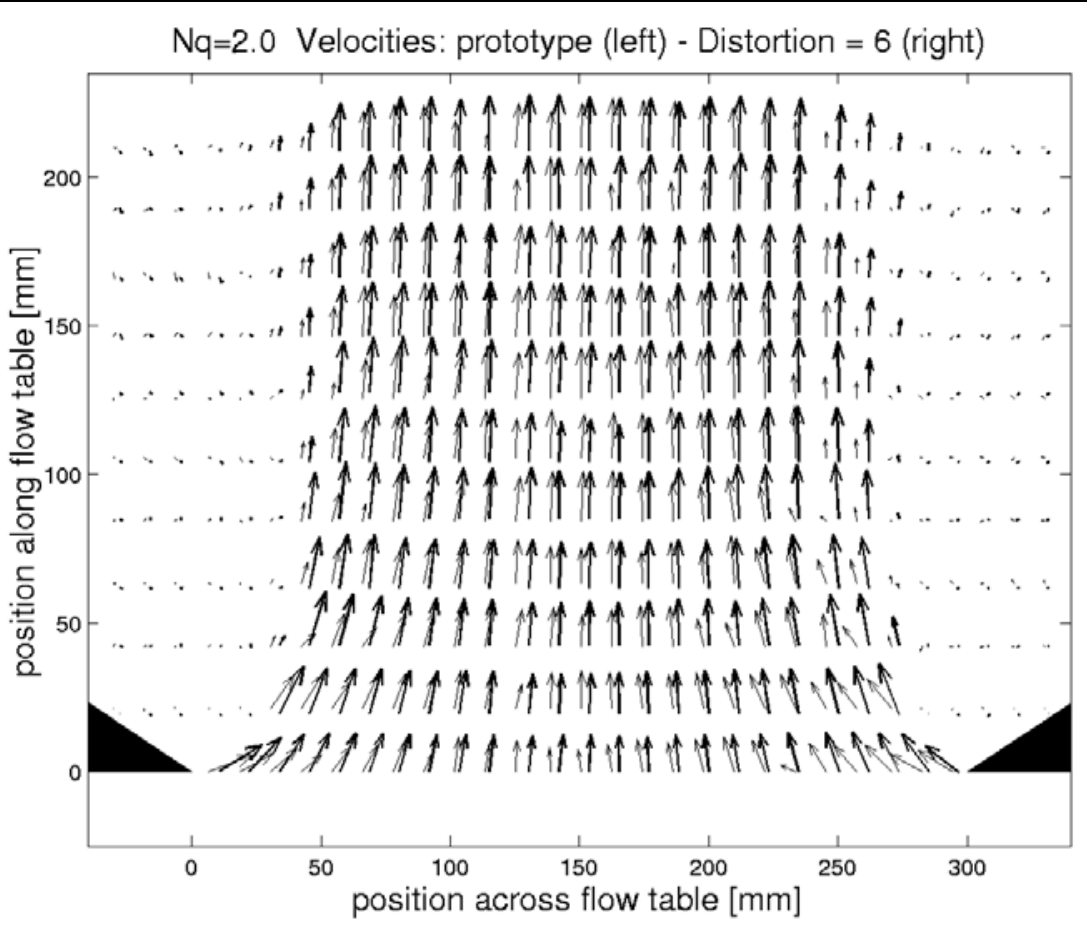

Figure $\mathrm{A} 31$. Case $1, Q=0.75 \mathrm{~L} / \mathrm{sec}$, prototype vs. distortion $=6$

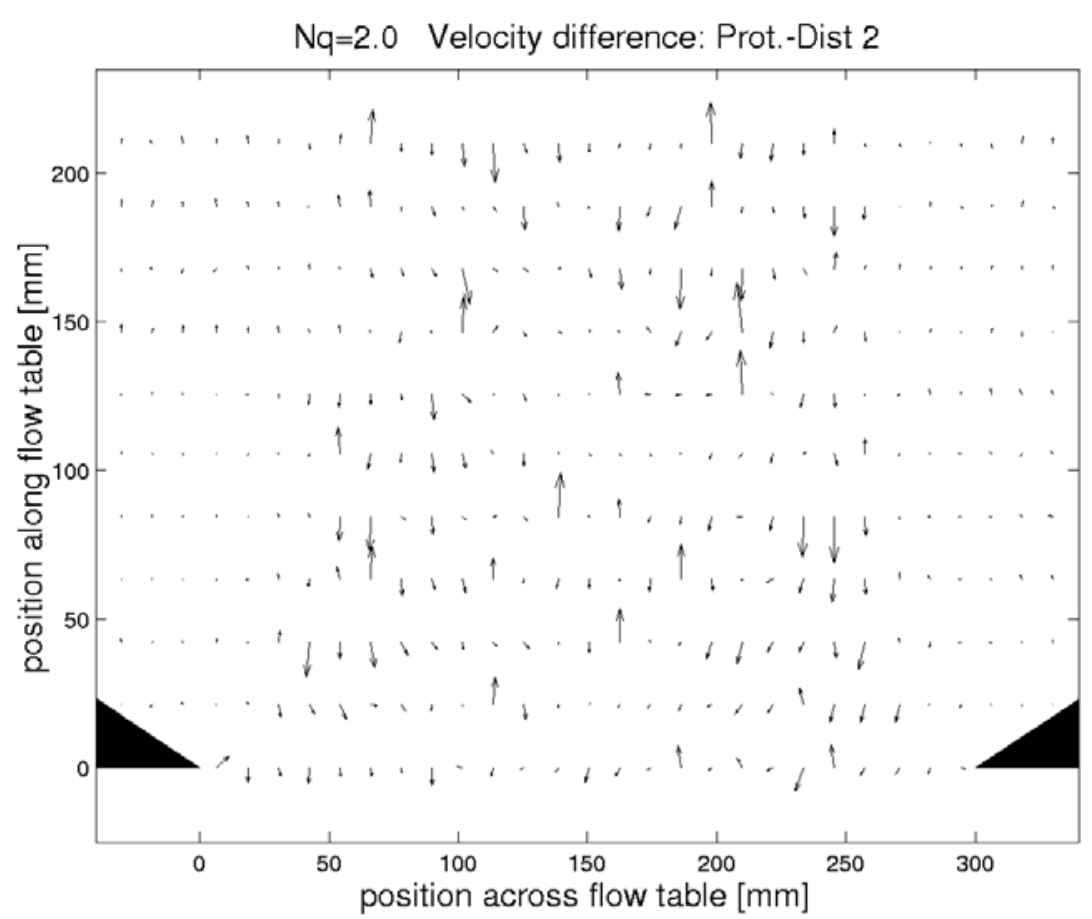

Figure A32. Case $1, Q=0.75 \mathrm{~L} / \mathrm{sec}$, prototype minus distortion $=2$ 


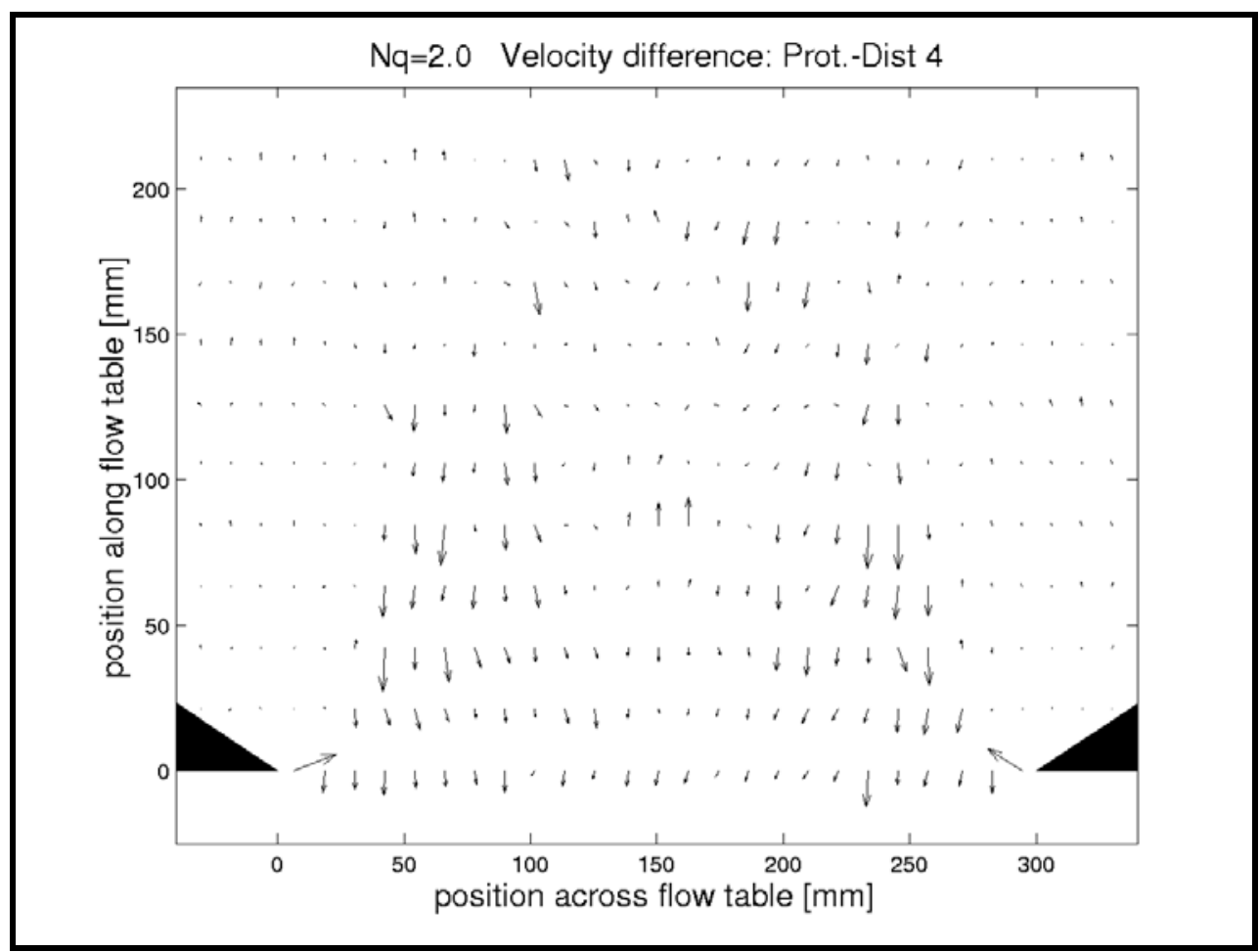

Figure A33. Case $1, Q=0.75 \mathrm{~L} / \mathrm{sec}$, prototype minus distortion $=4$

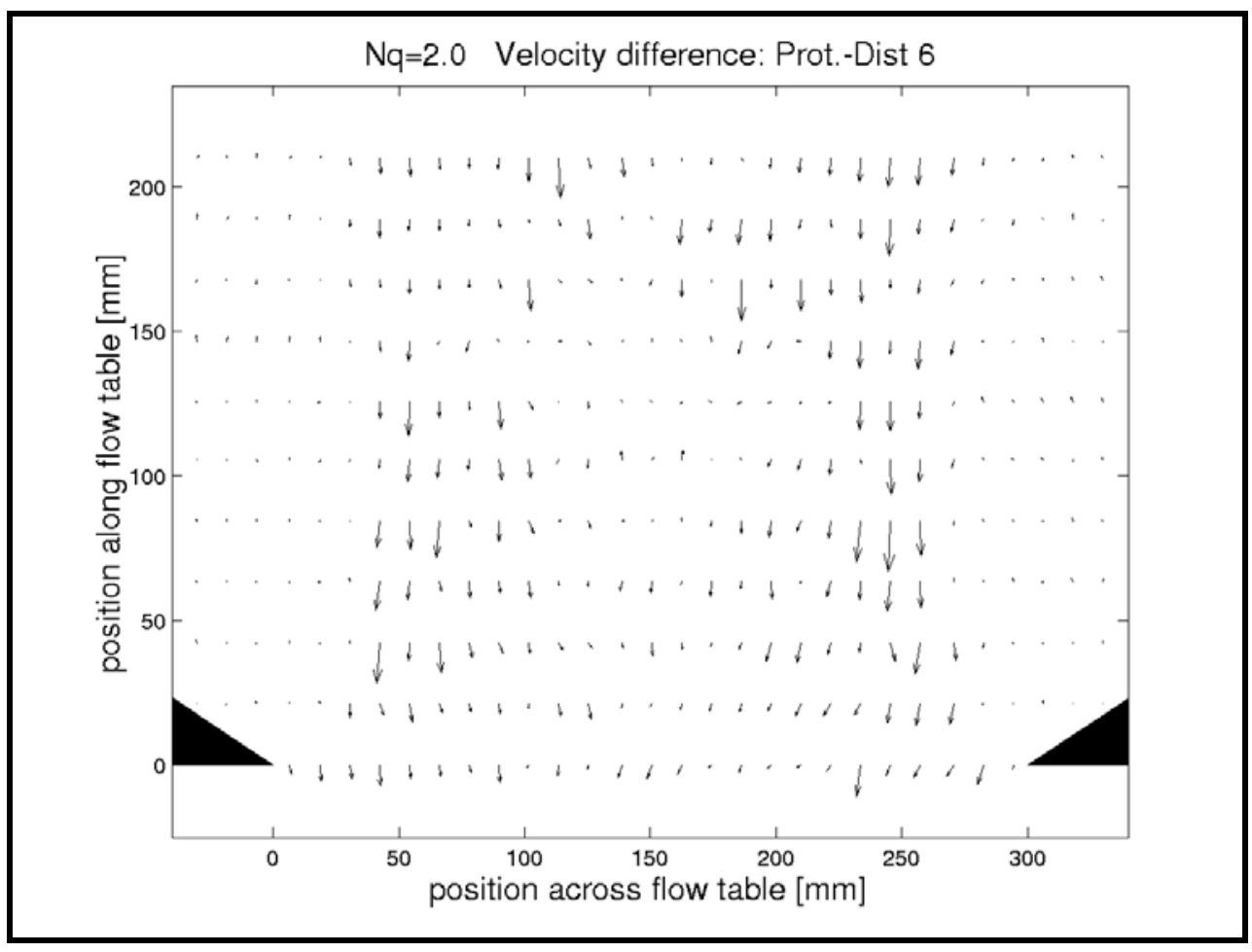

Figure A34. Case $1, Q=0.75 \mathrm{~L} / \mathrm{sec}$, prototype minus distortion $=6$ 


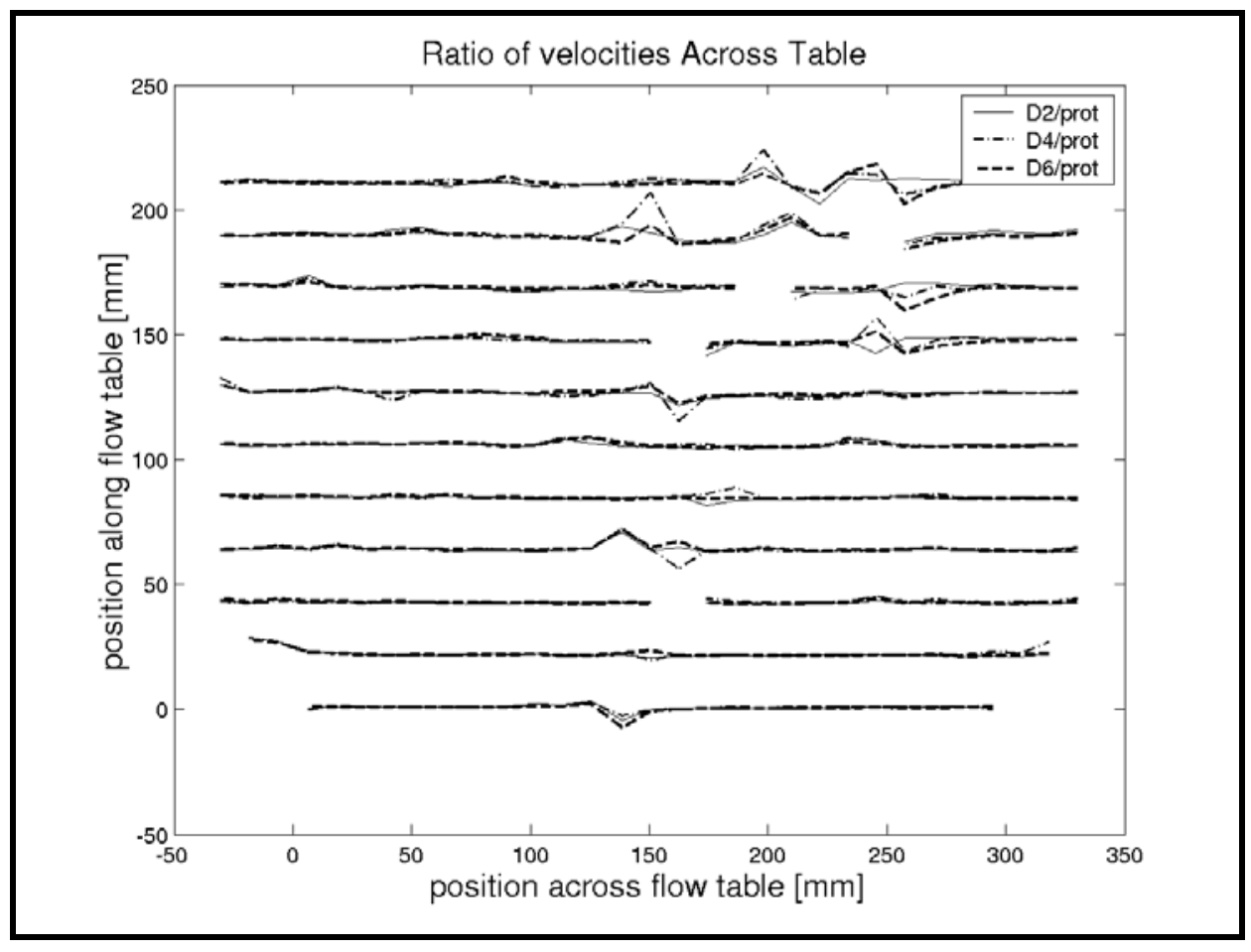

Figure $\mathrm{A} 35$. Case $1, Q=0.75 \mathrm{~L} / \mathrm{sec}$, ratios between prototype and distortions 2, 4 , and 6

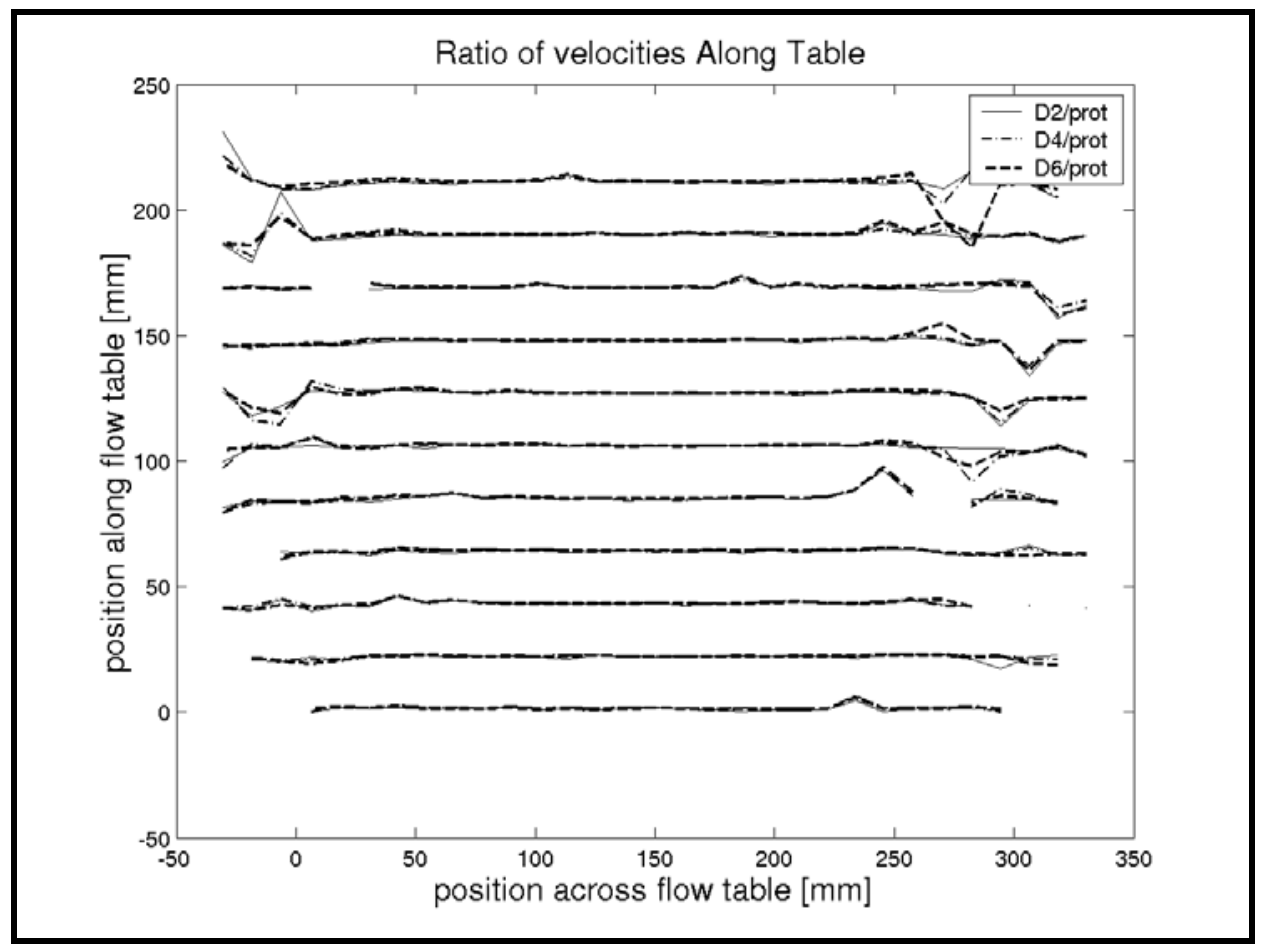

Figure A36. Case $1, Q=0.75 \mathrm{~L} / \mathrm{sec}$, ratios between prototype and distortions 2 , 4 , and 6 


\section{Appendix B \\ Case 2 - Flow Separation at Vertical Edge - Constrained Jet}

This Appendix contains the complete set of comparisons between prototype and geometrically-distorted models for the case of flow separation on the boundary of a constrained jet created by a gap with vertical edges. The free jet was constrained by parallel jetties downstream of the gap. See Chapter 5 for a description of the experiments and discussion of results. 


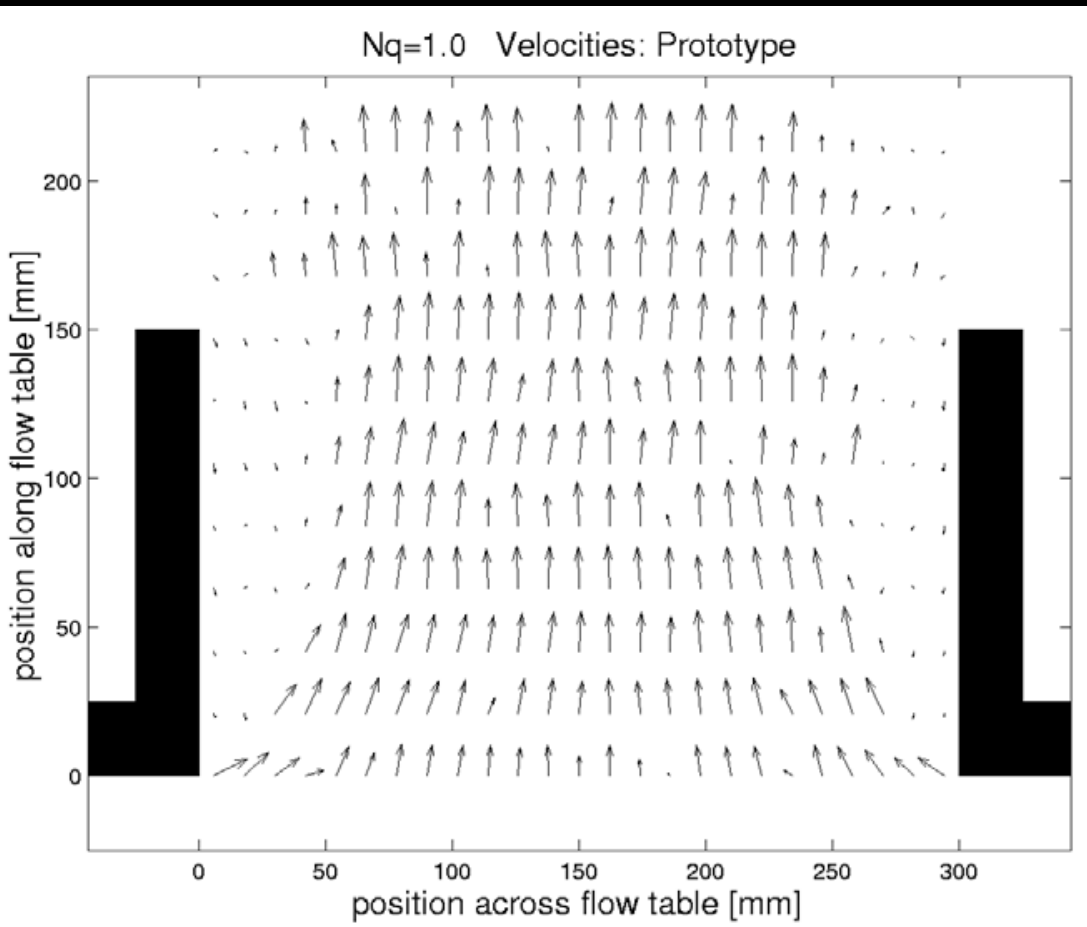

Figure B1. Case 2, $Q=1.5 \mathrm{~L} / \mathrm{sec}$, prototype

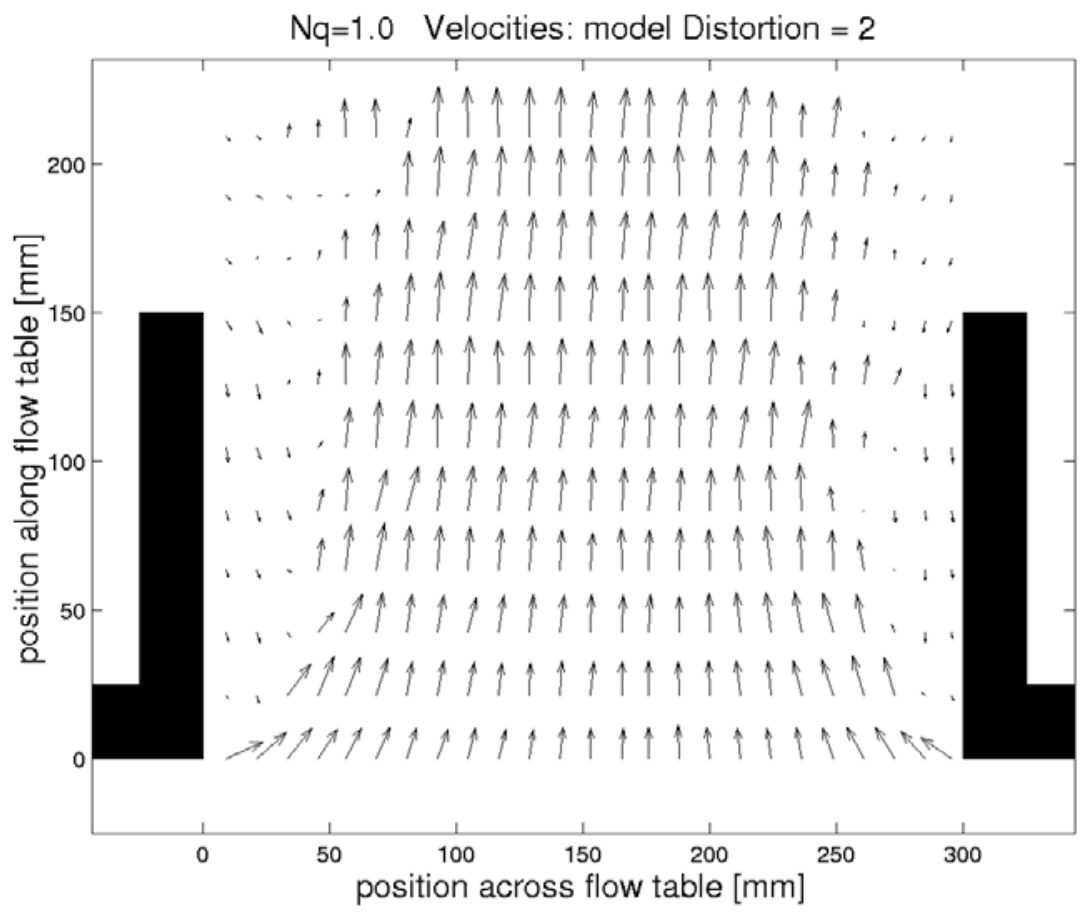

Figure B2. Case 2, $Q=1.5 \mathrm{~L} / \mathrm{sec}$, distortion $=2$ 


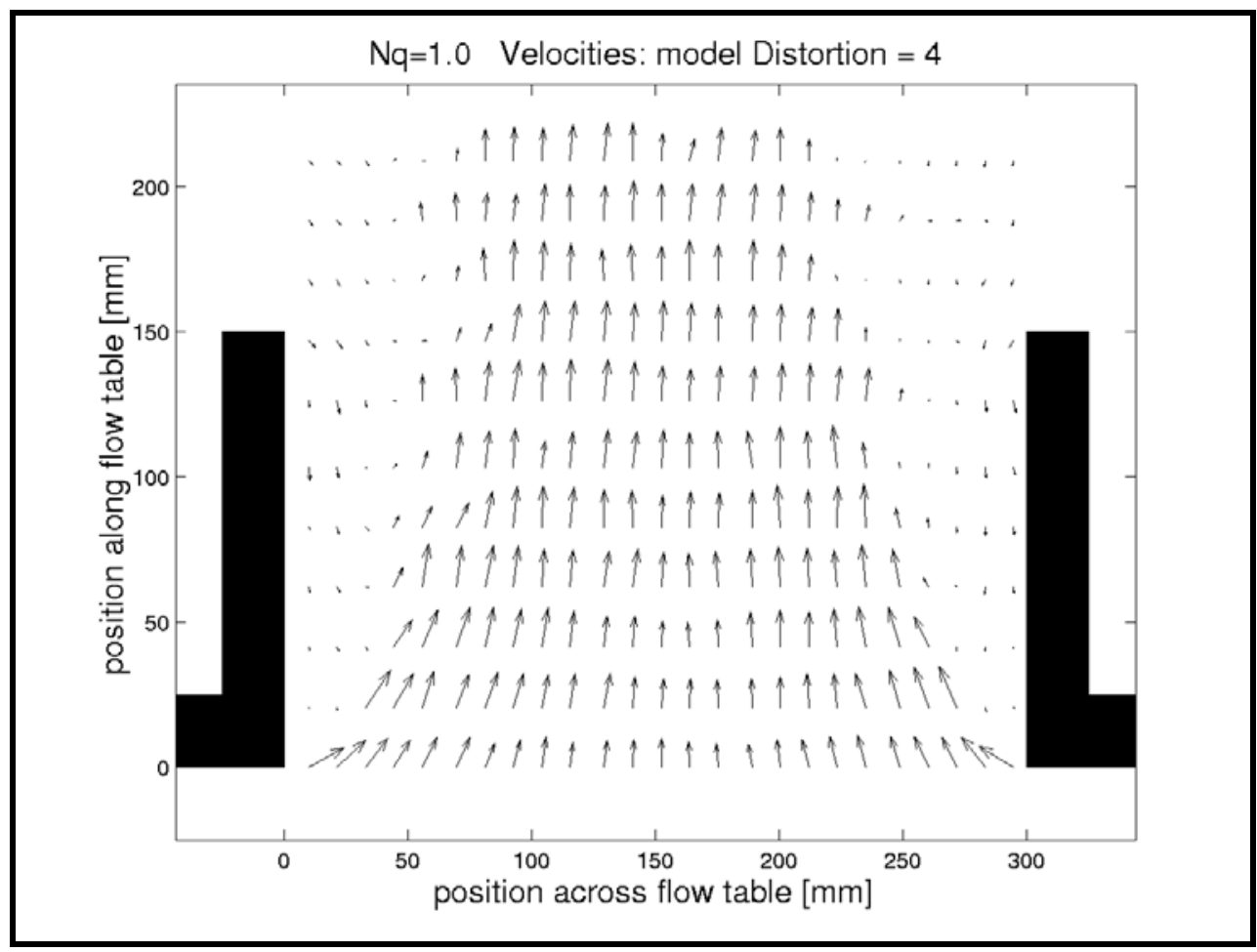

Figure B3. Case 2, $Q=1.5 \mathrm{~L} / \mathrm{sec}$, distortion $=4$

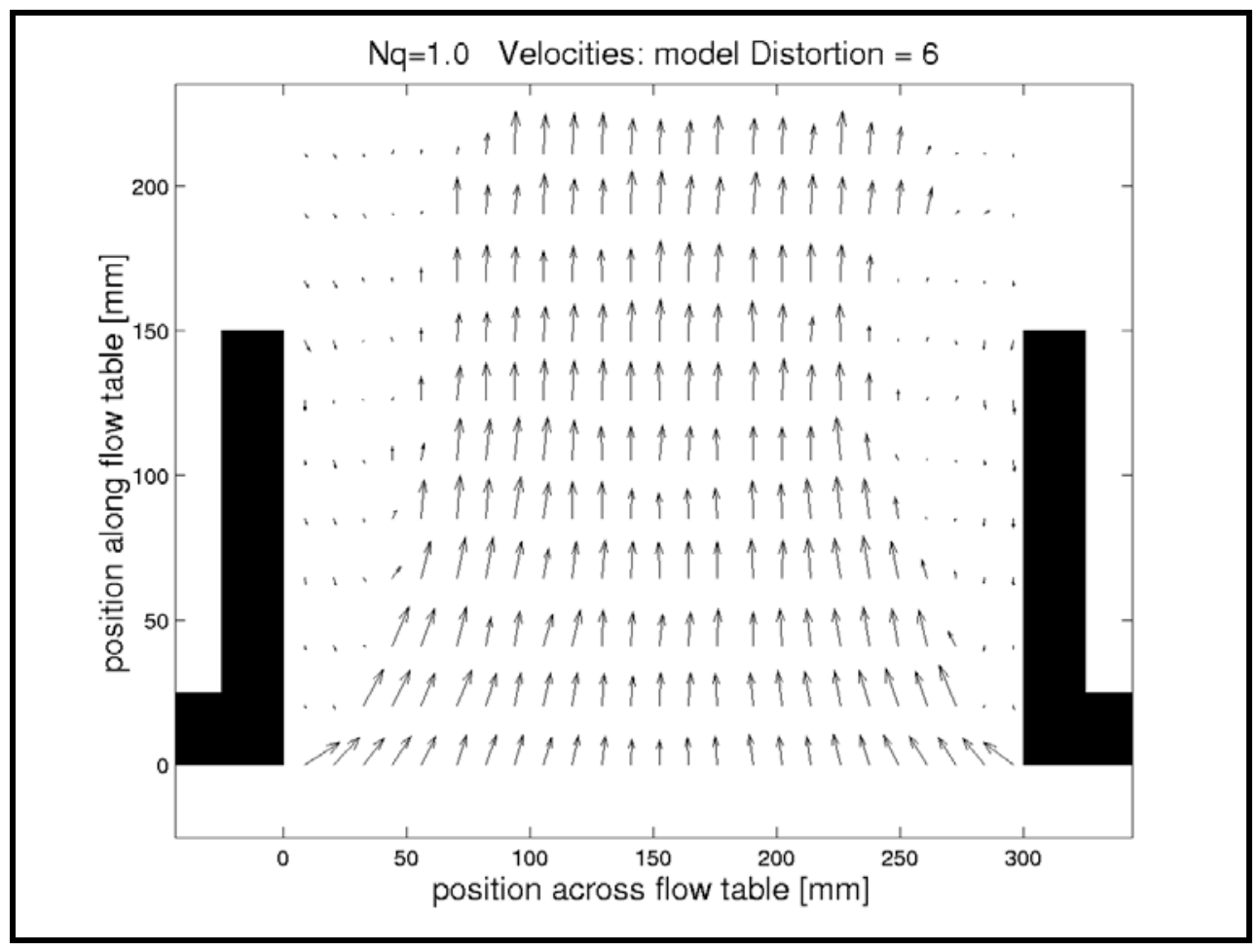

Figure B4. Case 2, $Q=1.5 \mathrm{~L} / \mathrm{sec}$, distortion $=6$ 


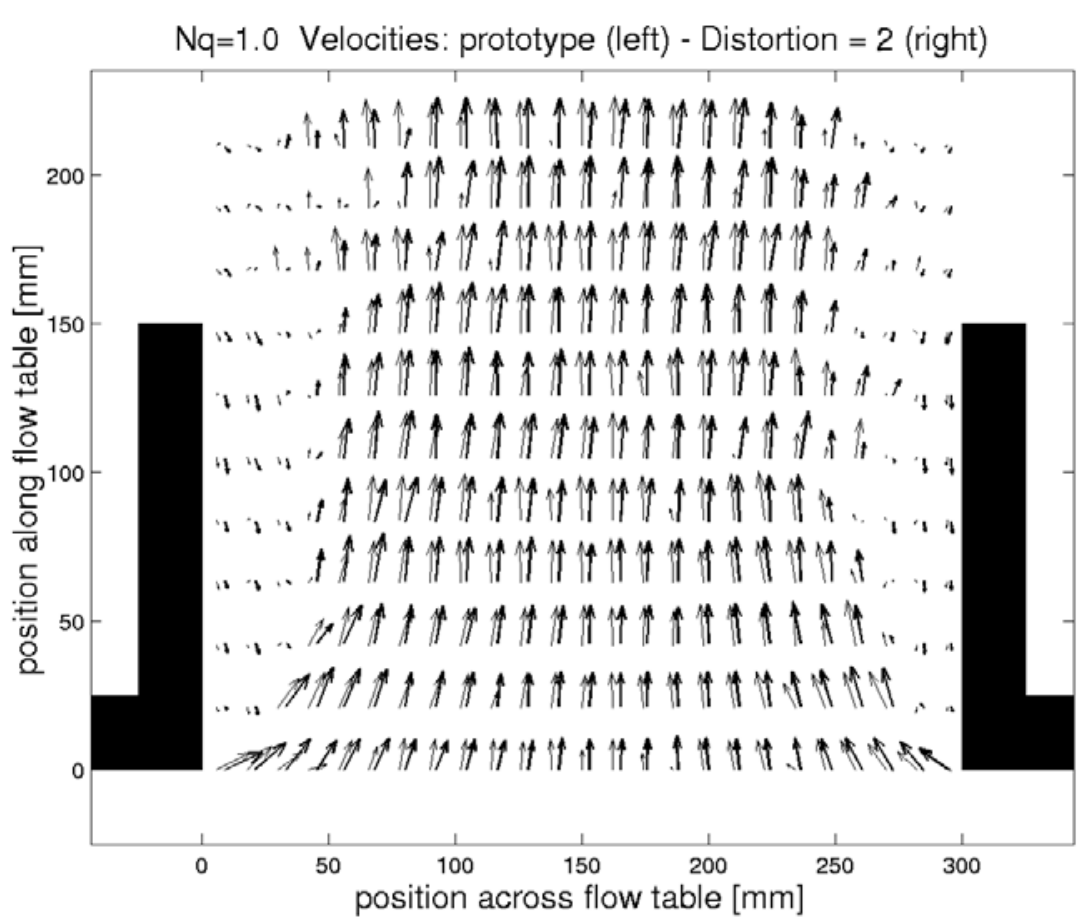

Figure B5. Case 2, $Q=1.5 \mathrm{~L} / \mathrm{sec}$, prototype vs. distortion $=2$

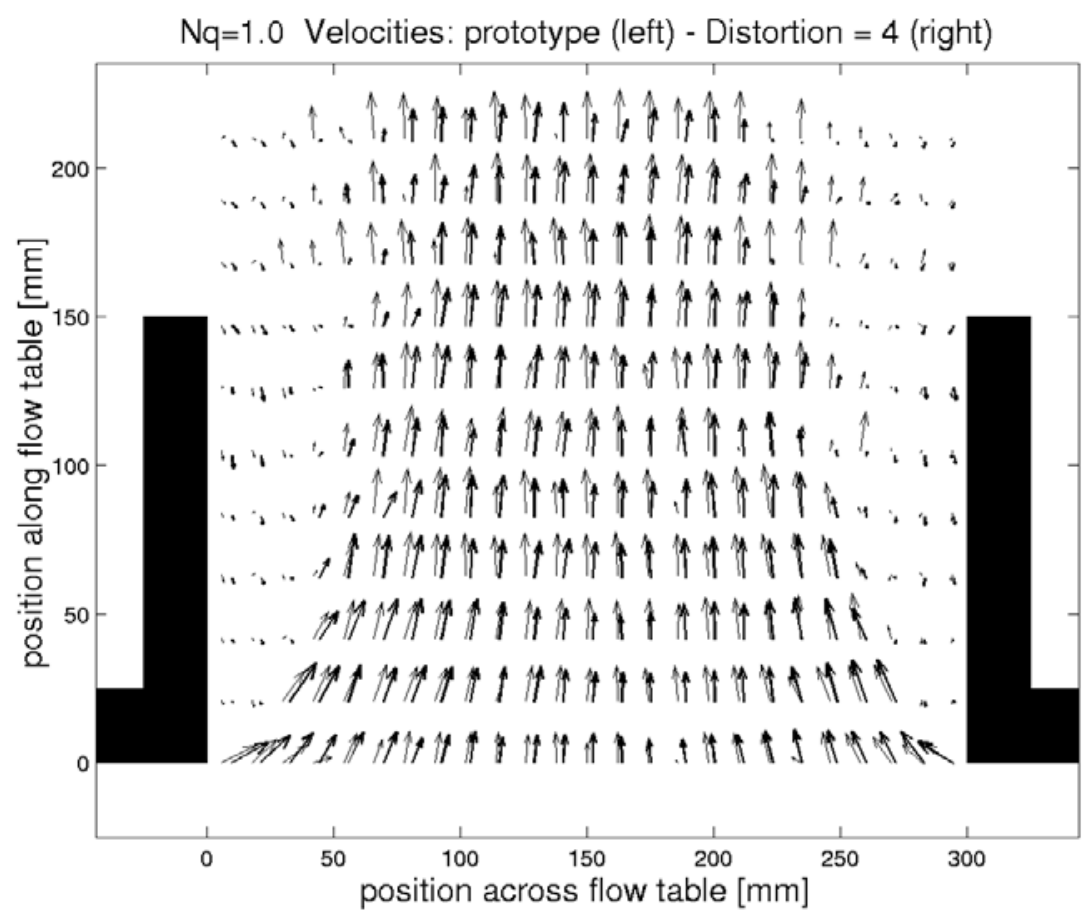

Figure B6. Case 2, $Q=1.5 \mathrm{~L} / \mathrm{sec}$, prototype vs. distortion $=4$ 


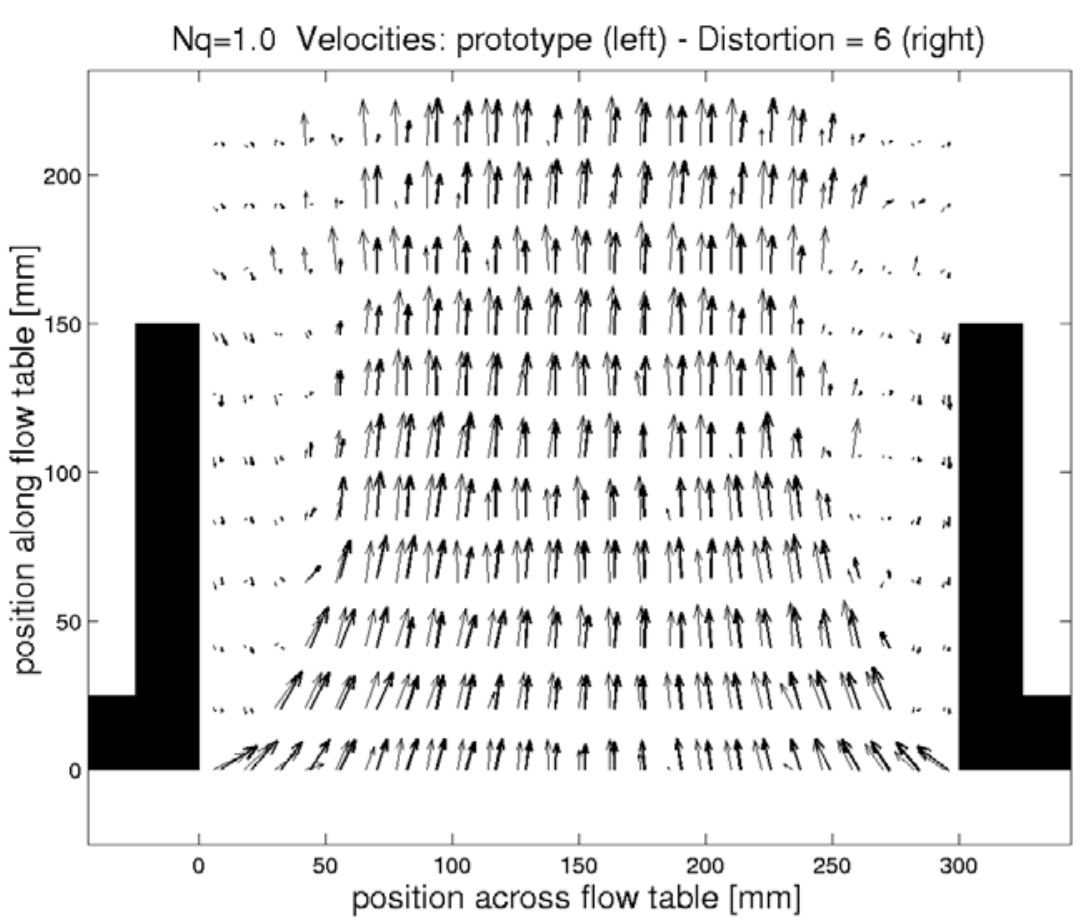

Figure B7. Case 2, $Q=1.5 \mathrm{~L} / \mathrm{sec}$, prototype vs. distortion $=6$

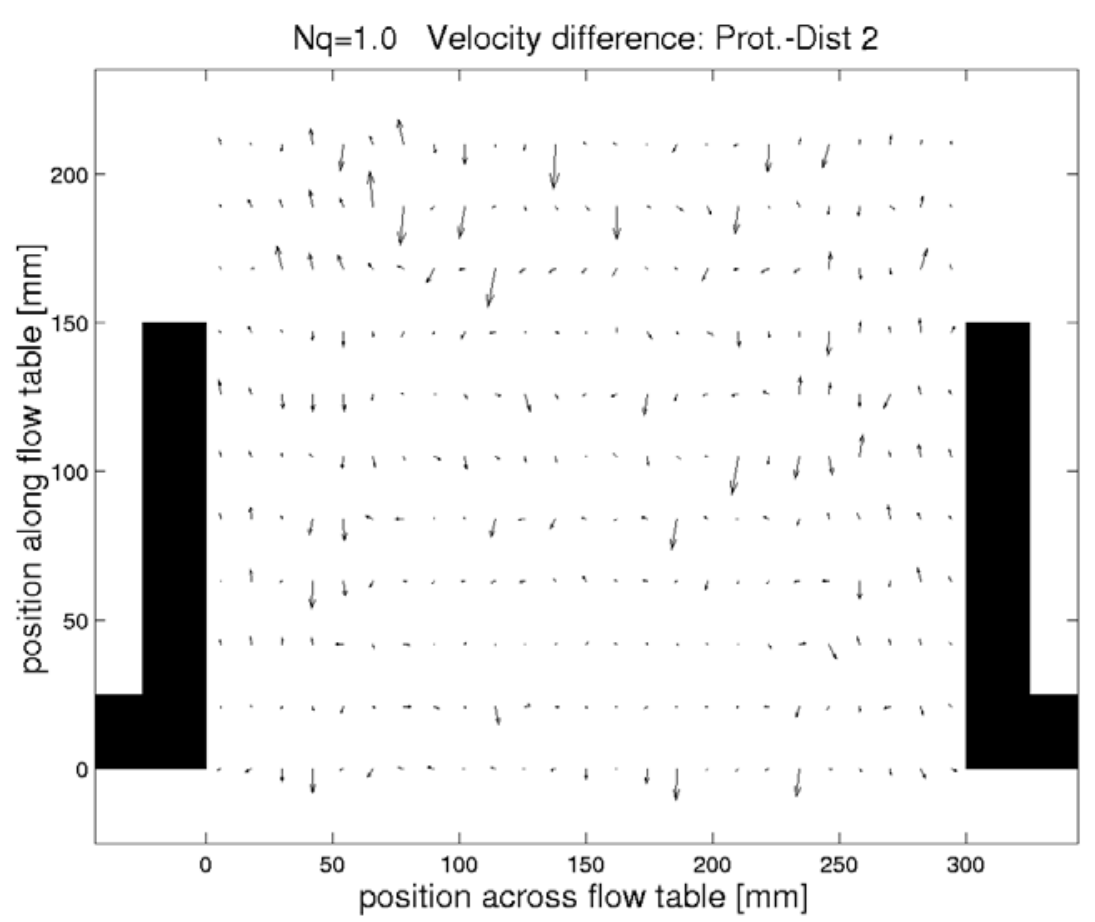

Figure B8. Case 2, $Q=1.5 \mathrm{~L} / \mathrm{sec}$, prototype minus distortion = 2 


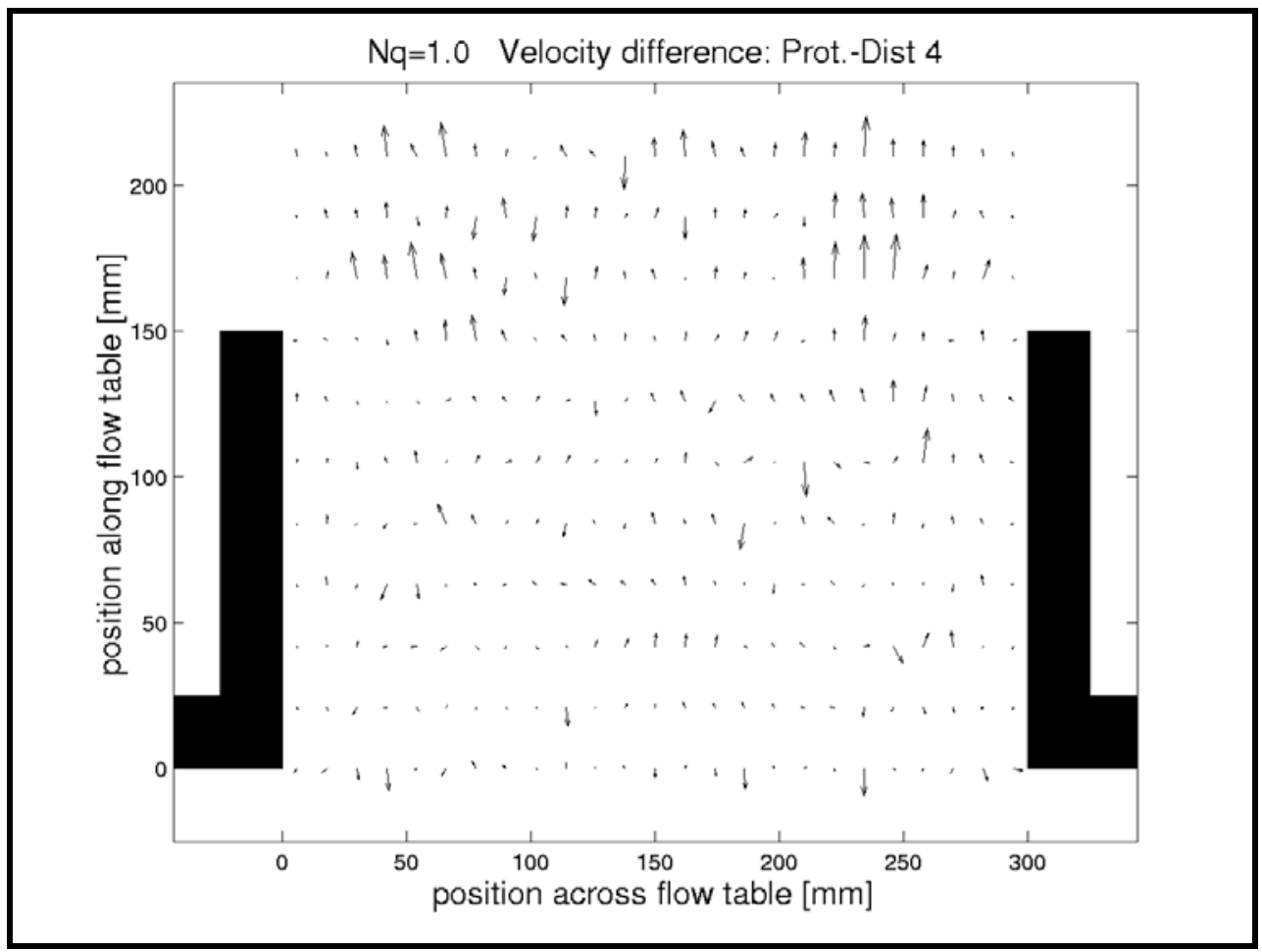

Figure B9. Case 2, $Q=1.5 \mathrm{~L} / \mathrm{sec}$, prototype minus distortion $=4$

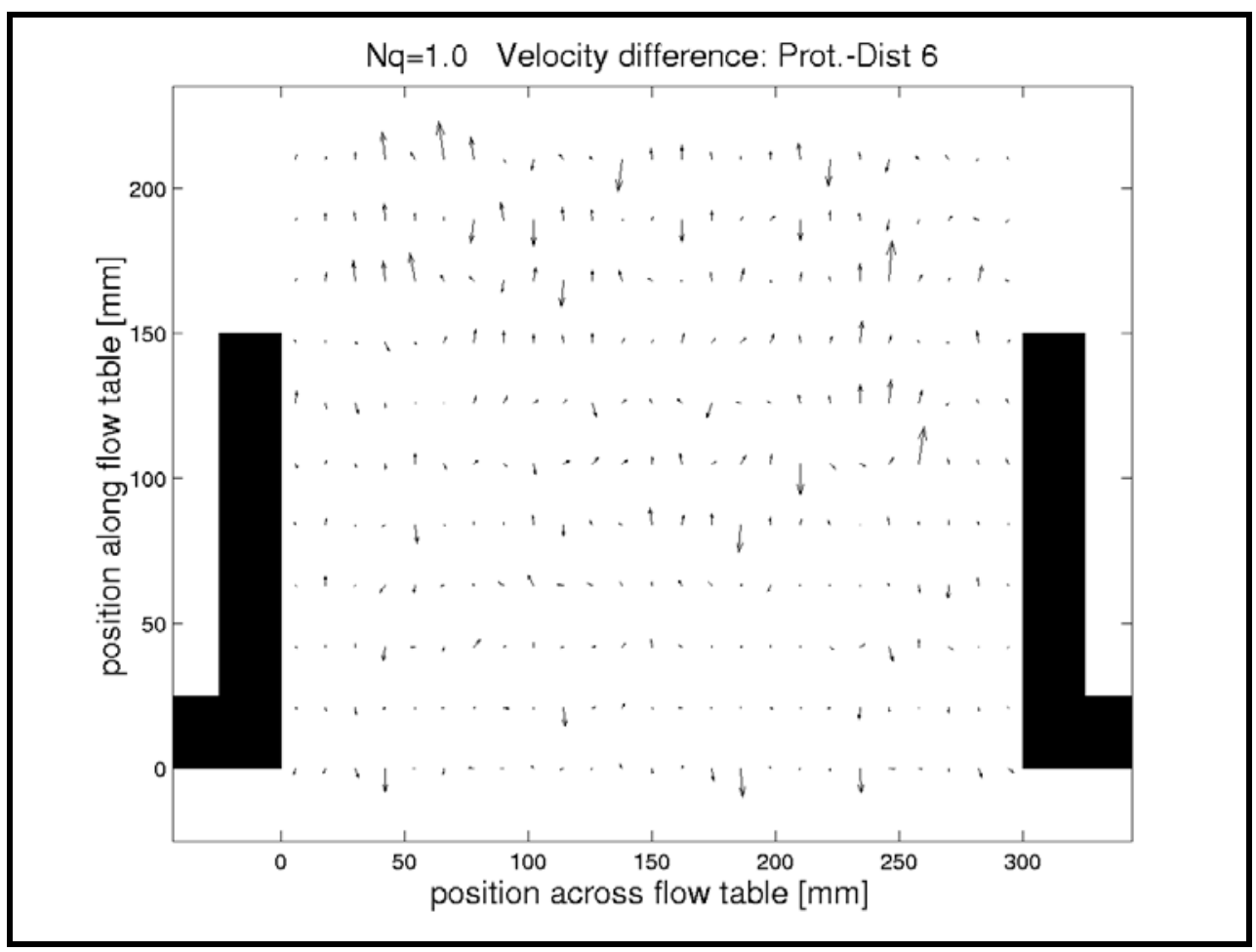

Figure B10. Case 2, $Q=1.5 \mathrm{~L} / \mathrm{sec}$, prototype minus distortion = 6 


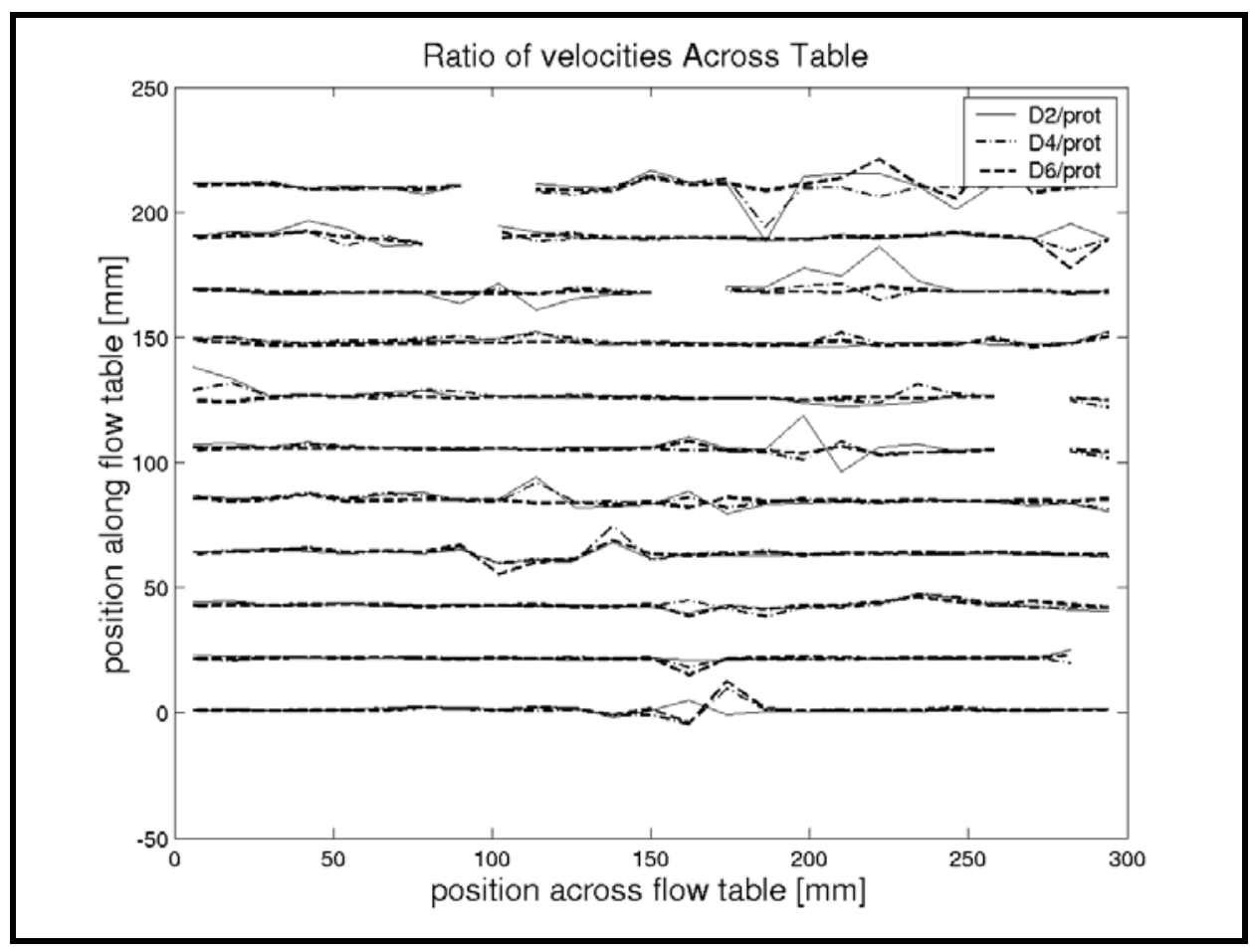

Figure B11. Case 2, $Q=1.5 \mathrm{~L} / \mathrm{sec}$, ratios between prototype and distortions 2, 4 , and 6

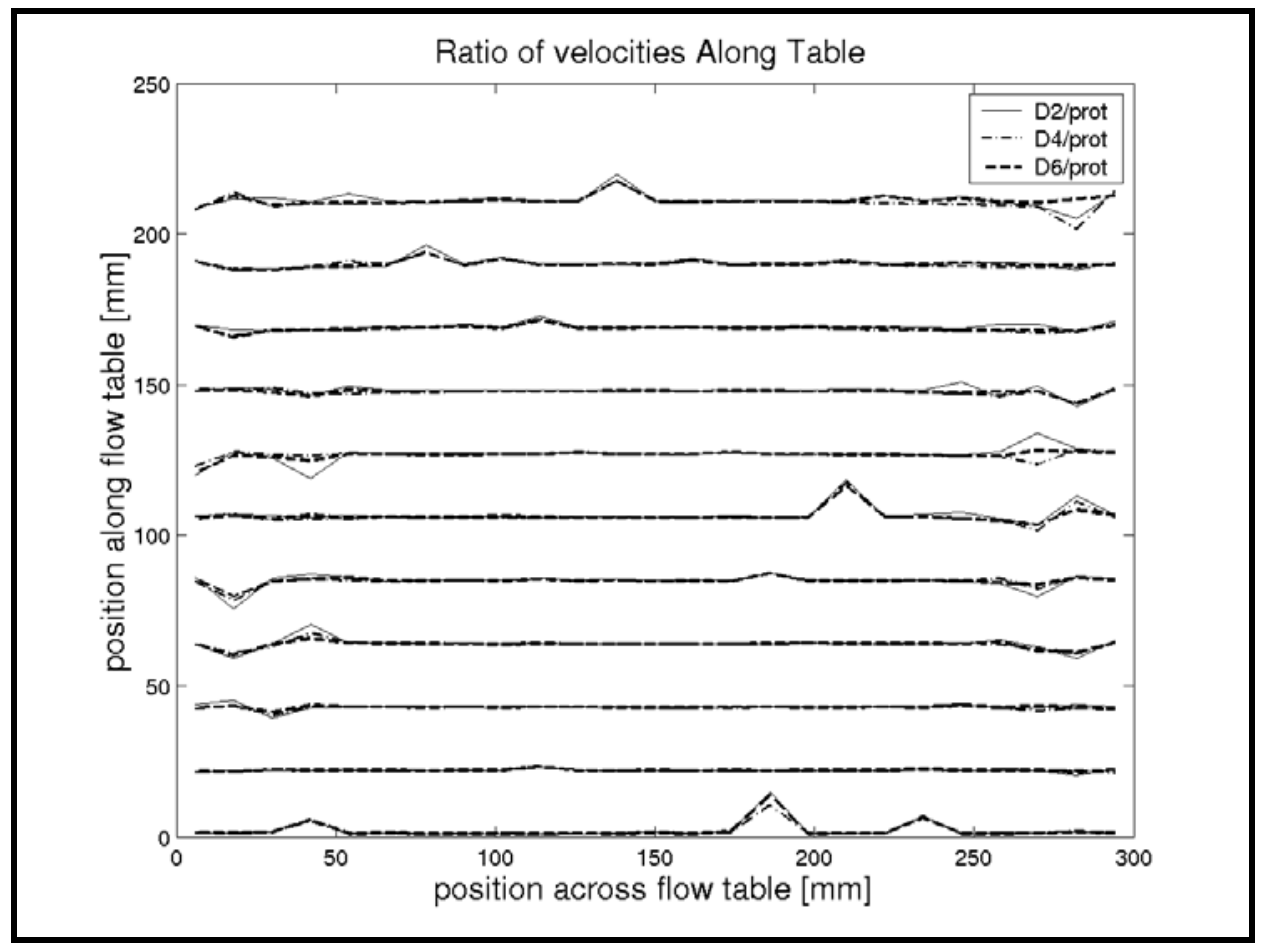

Figure B12. Case 2, $Q=1.5 \mathrm{~L} / \mathrm{sec}$, ratios between prototype and distortions 2, 4 , and 6 


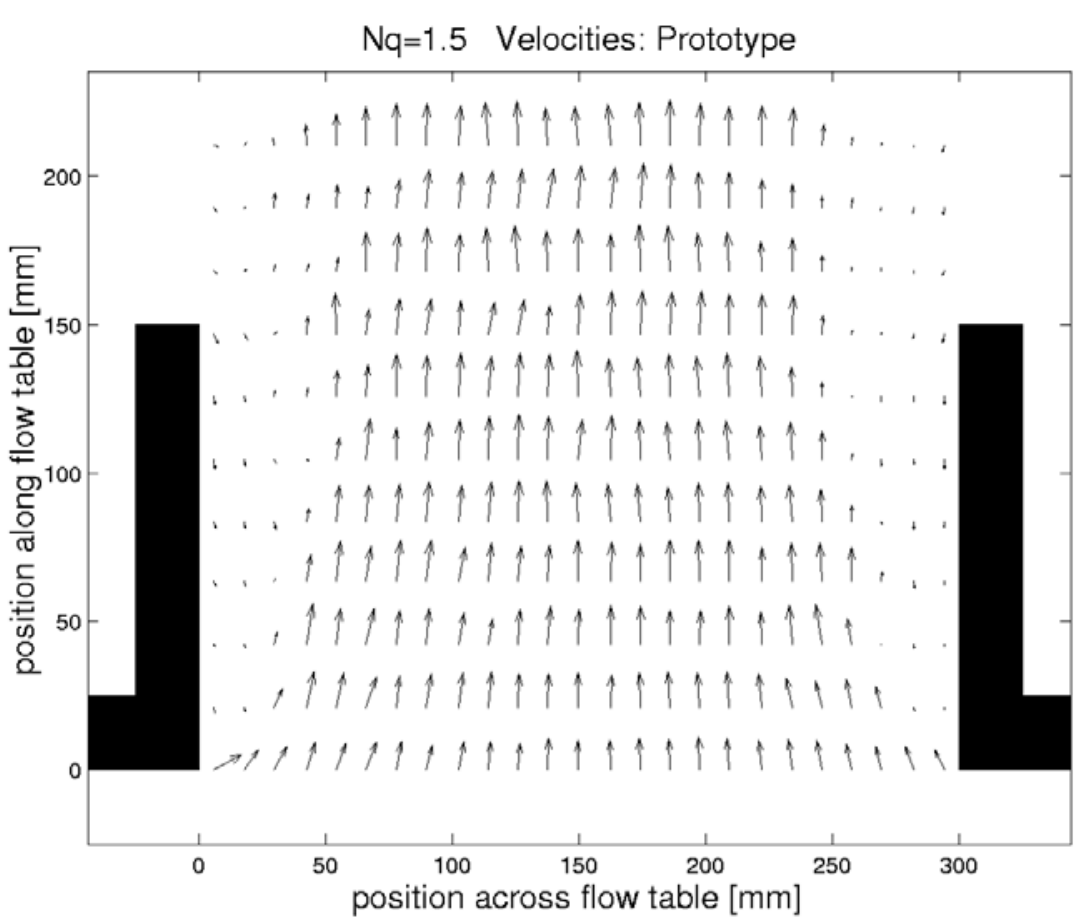

Figure B13. Case 2, $Q=1.0 \mathrm{~L} / \mathrm{sec}$, prototype

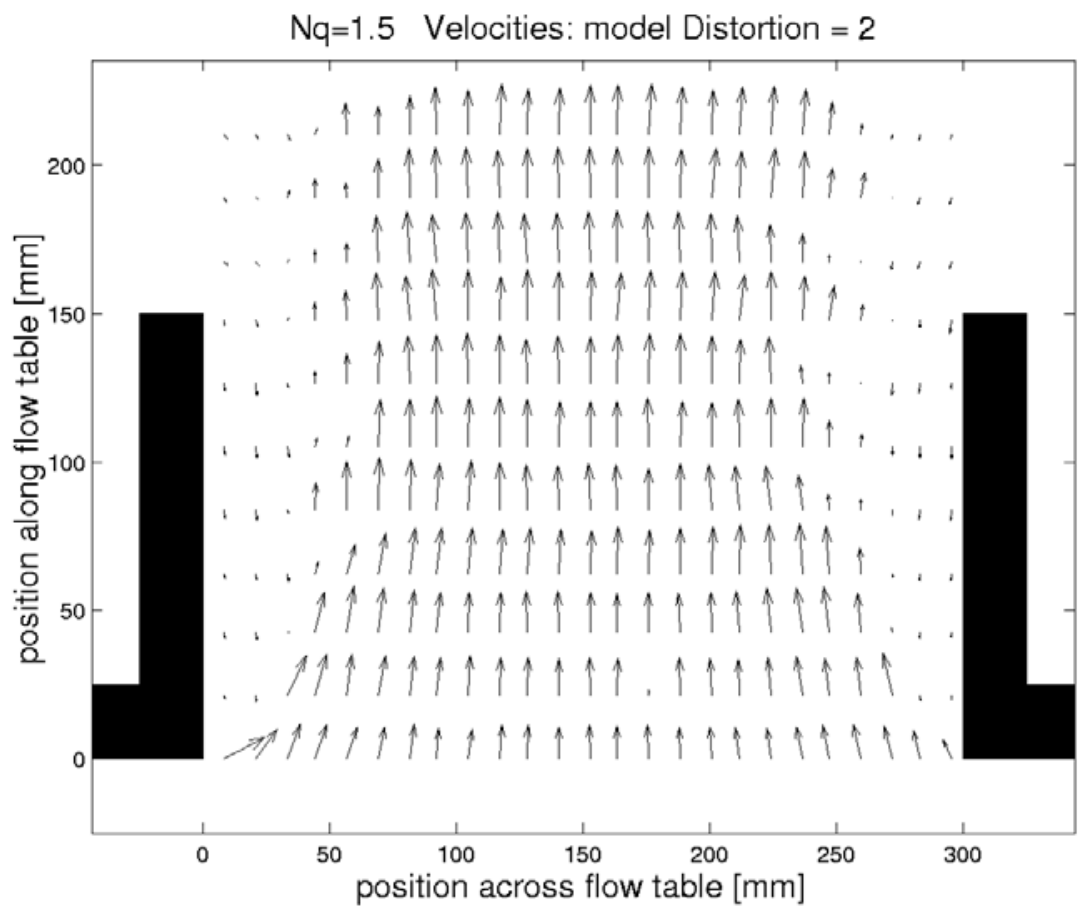

Figure B14. Case 2, $Q=1.0 \mathrm{~L} / \mathrm{sec}$, distortion $=2$ 


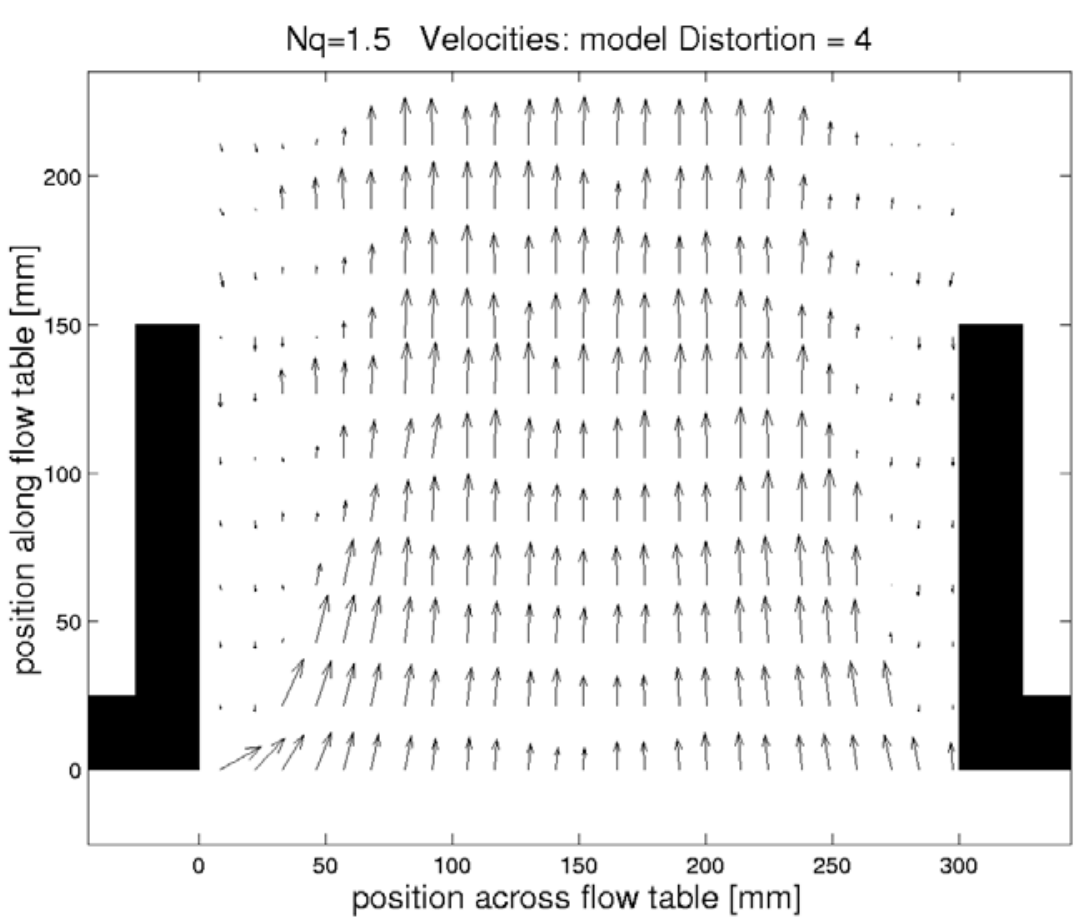

Figure B15. Case 2, $Q=1.0 \mathrm{~L} / \mathrm{sec}$, distortion $=4$

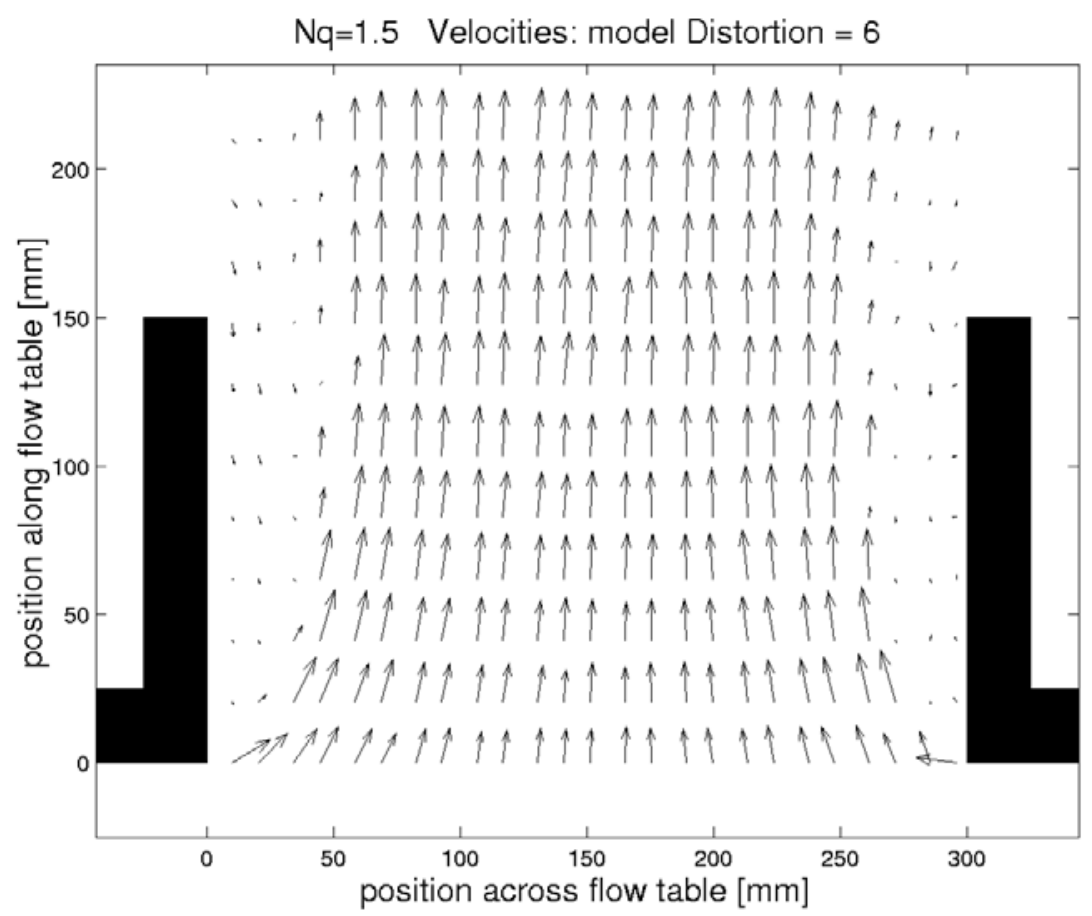

Figure B16. Case 2, $Q=1.0 \mathrm{~L} / \mathrm{sec}$, distortion $=6$ 


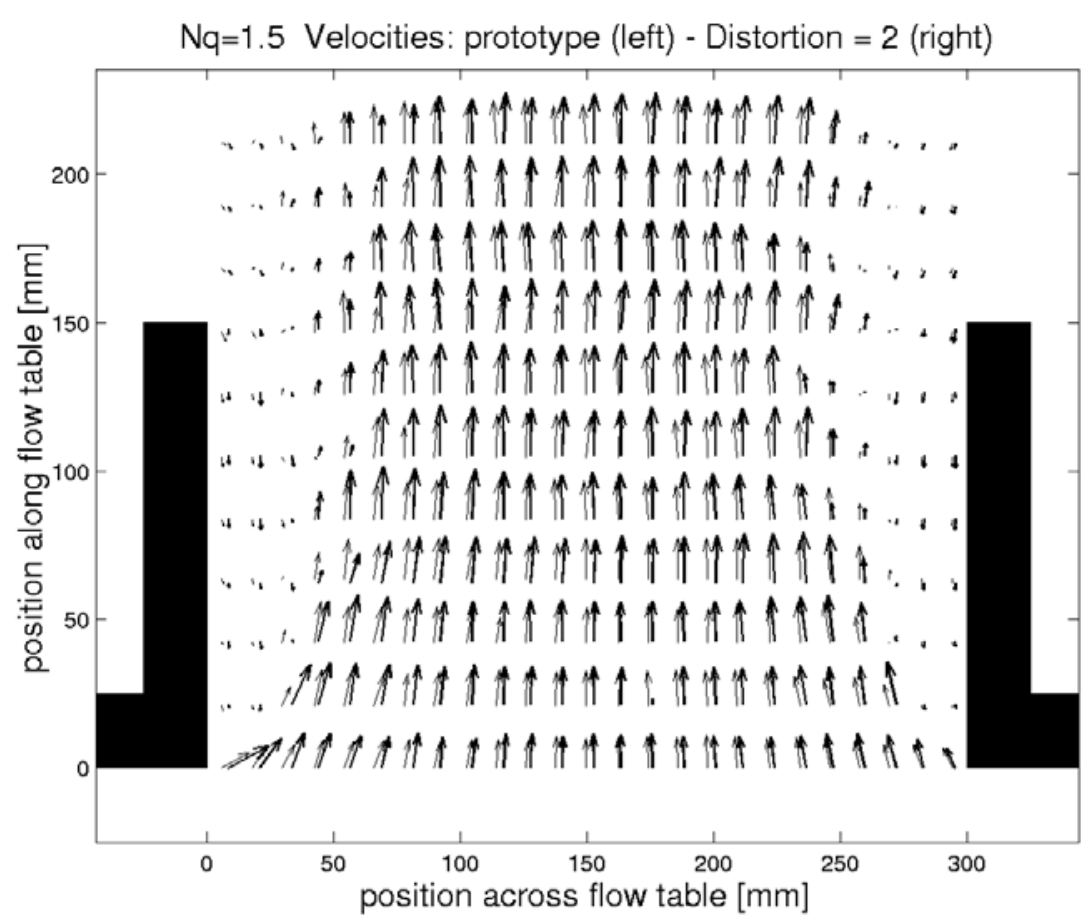

Figure B17. Case 2, $Q=1.0 \mathrm{~L} / \mathrm{sec}$, prototype vs. distortion $=2$

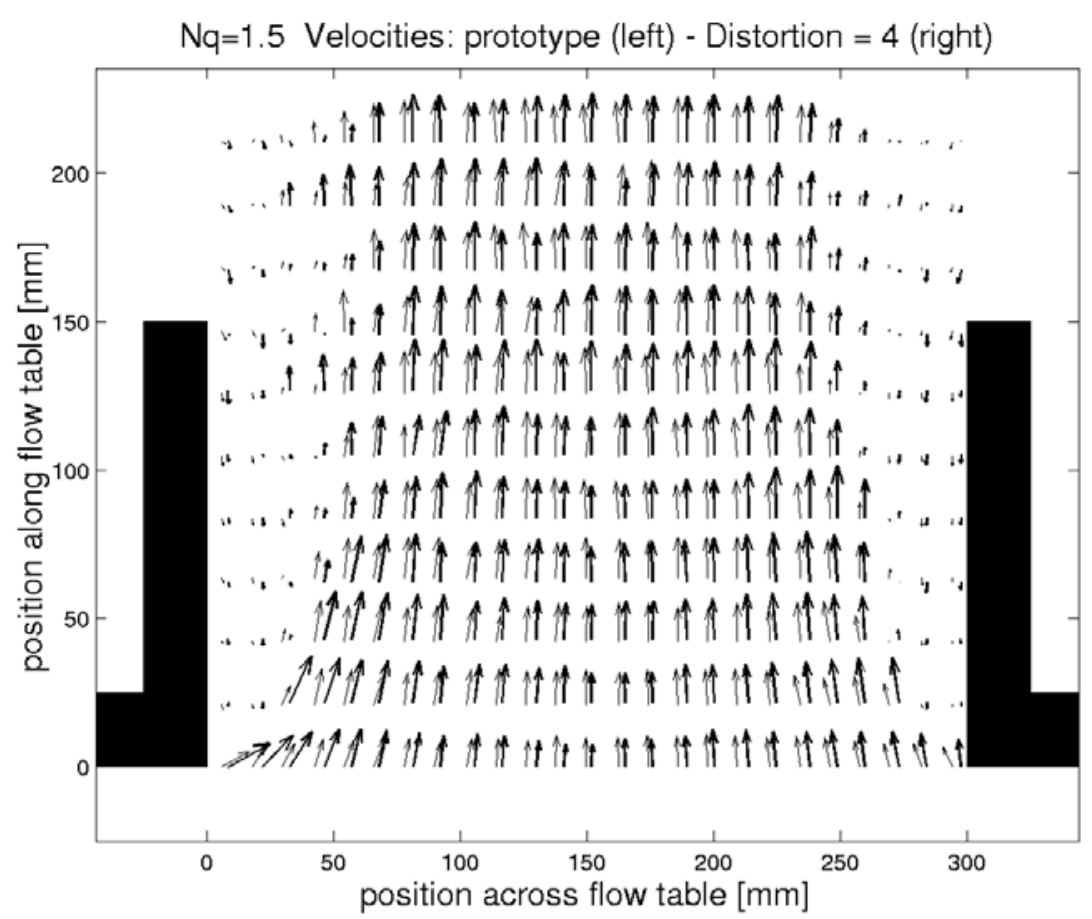

Figure B18. Case 2, $Q=1.0 \mathrm{~L} / \mathrm{sec}$, prototype vs. distortion $=4$ 


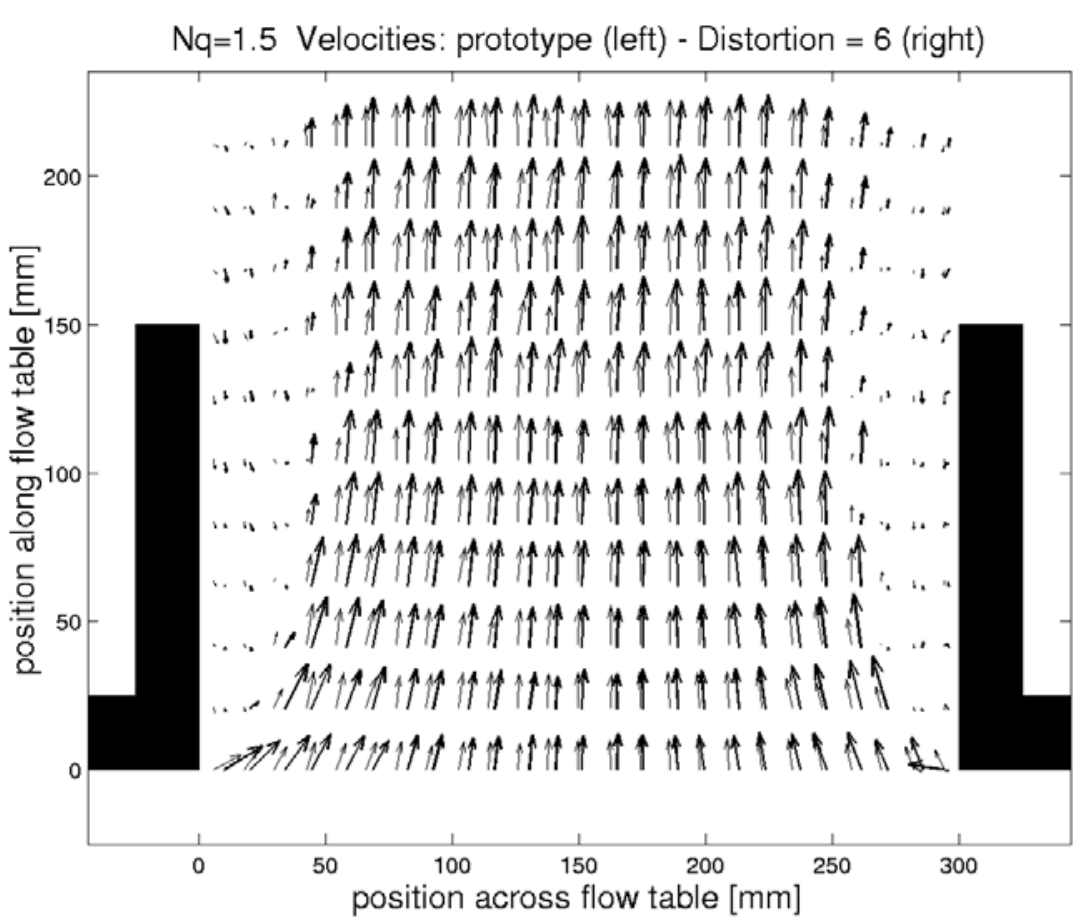

Figure B19. Case 2, $Q=1.0 \mathrm{~L} / \mathrm{sec}$, prototype vs. distortion $=6$

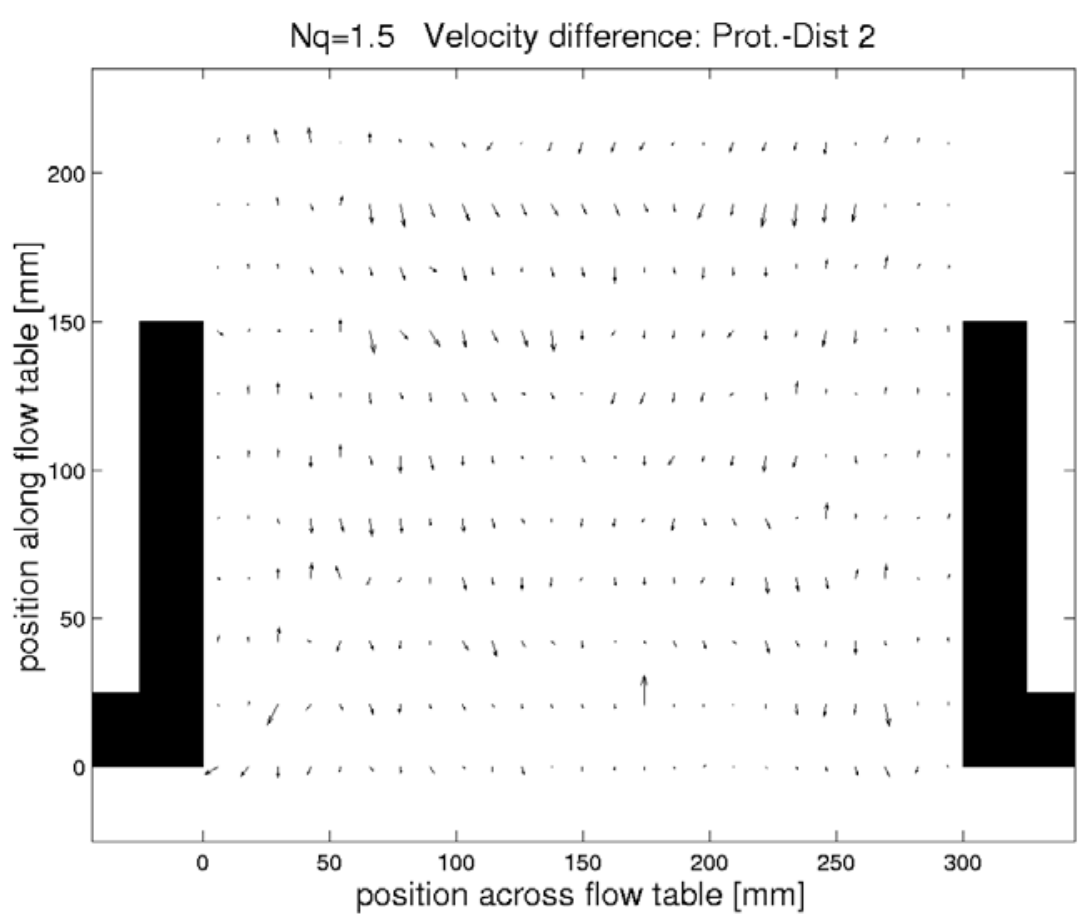

Figure B20. Case 2, $Q=1.0 \mathrm{~L} / \mathrm{sec}$, prototype minus distortion $=2$ 


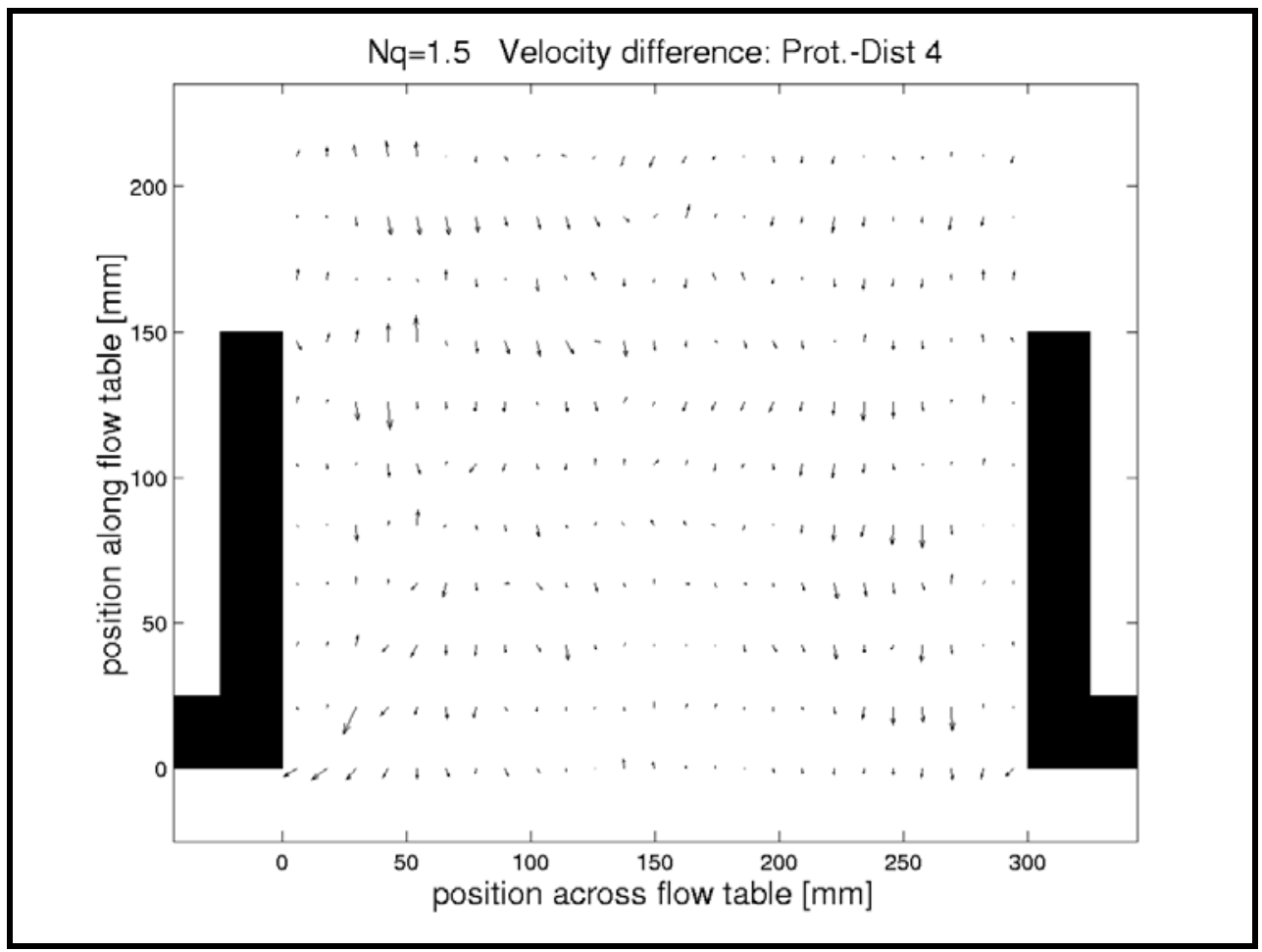

Figure B21. Case 2, $Q=1.0 \mathrm{~L} / \mathrm{sec}$, prototype minus distortion $=4$

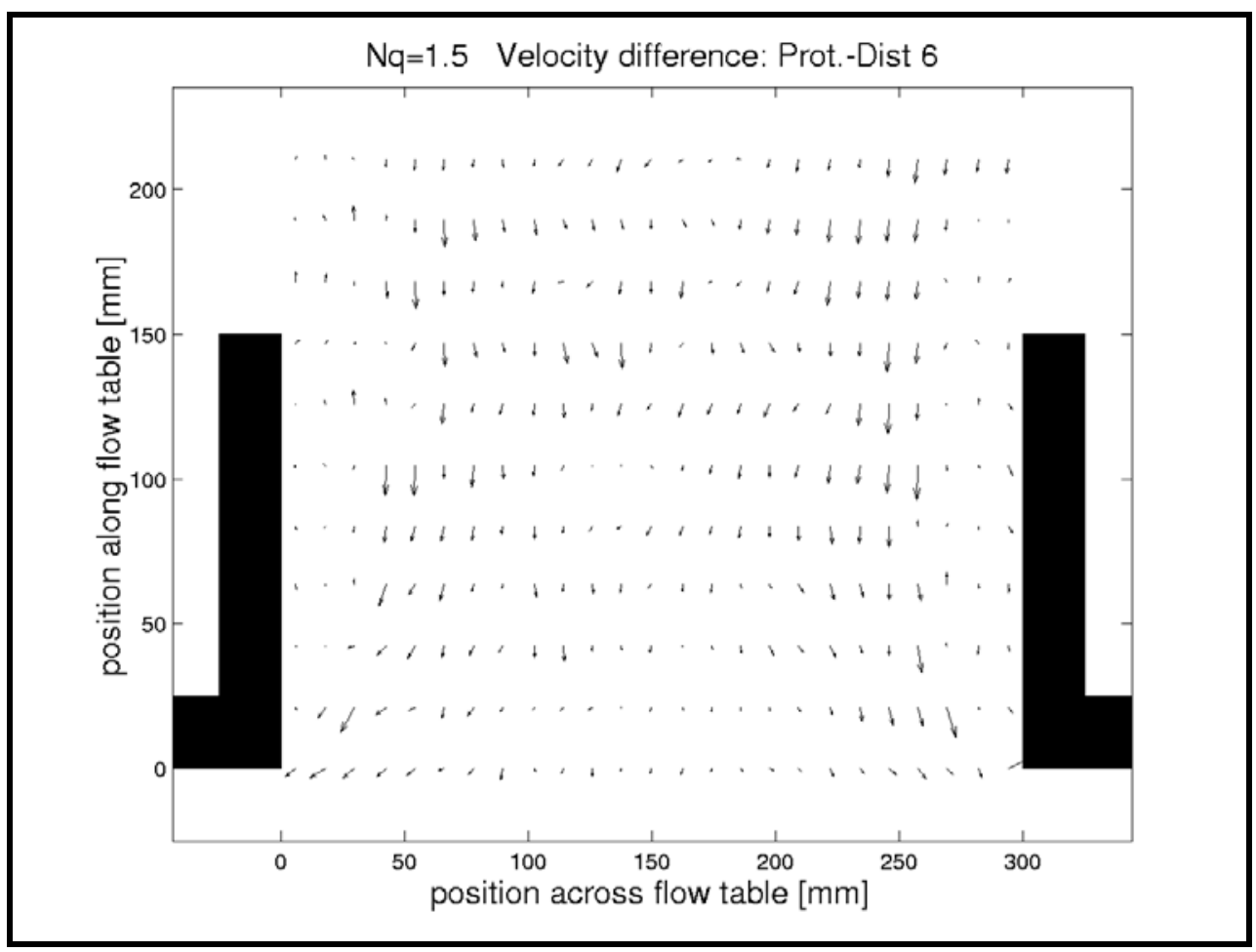

Figure B22. Case 2, $Q=1.0 \mathrm{~L} / \mathrm{sec}$, prototype minus distortion $=6$ 


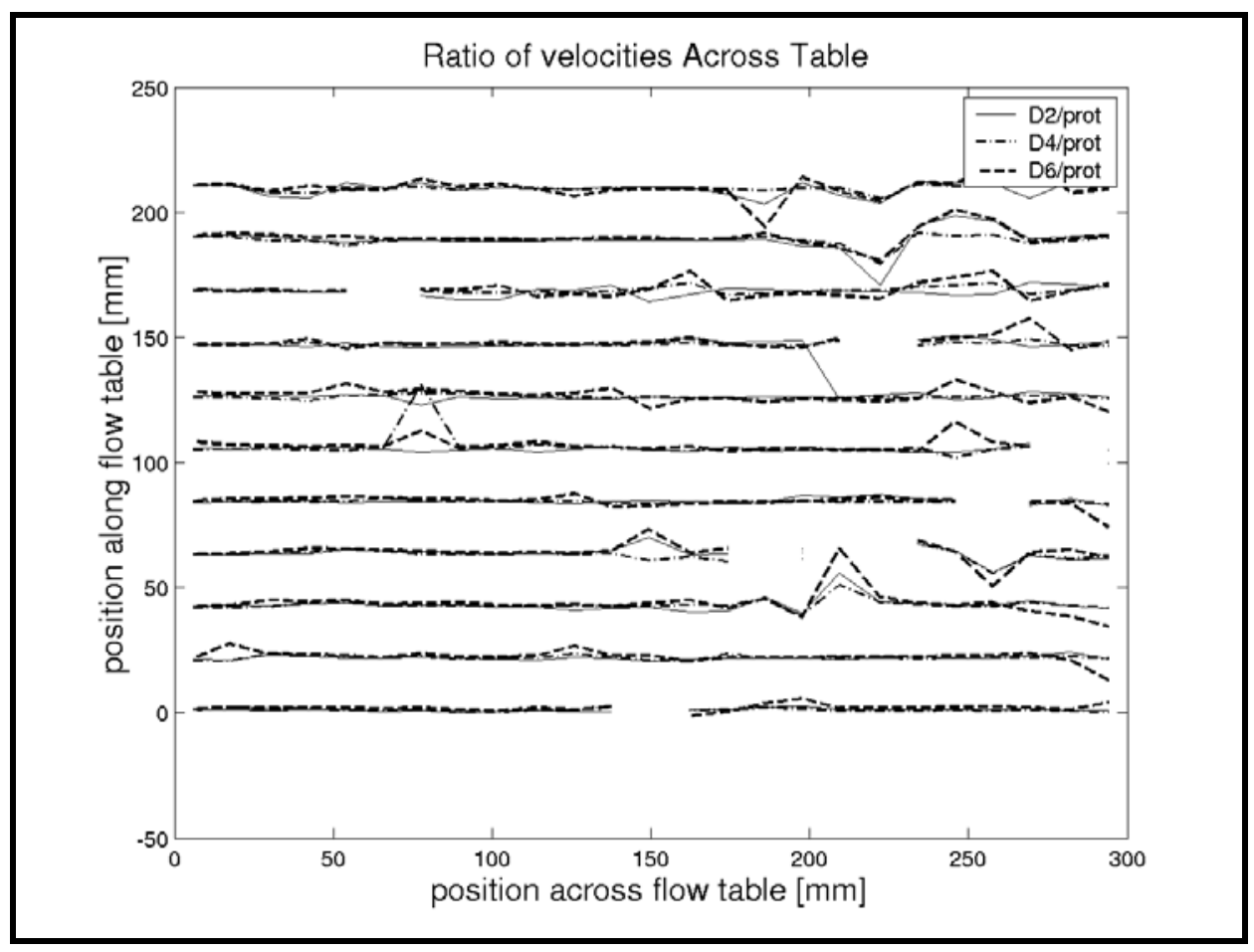

Figure B23. Case 2, $Q=1.0 \mathrm{~L} / \mathrm{sec}$, ratios between prototype and distortions 2, 4 , and 6

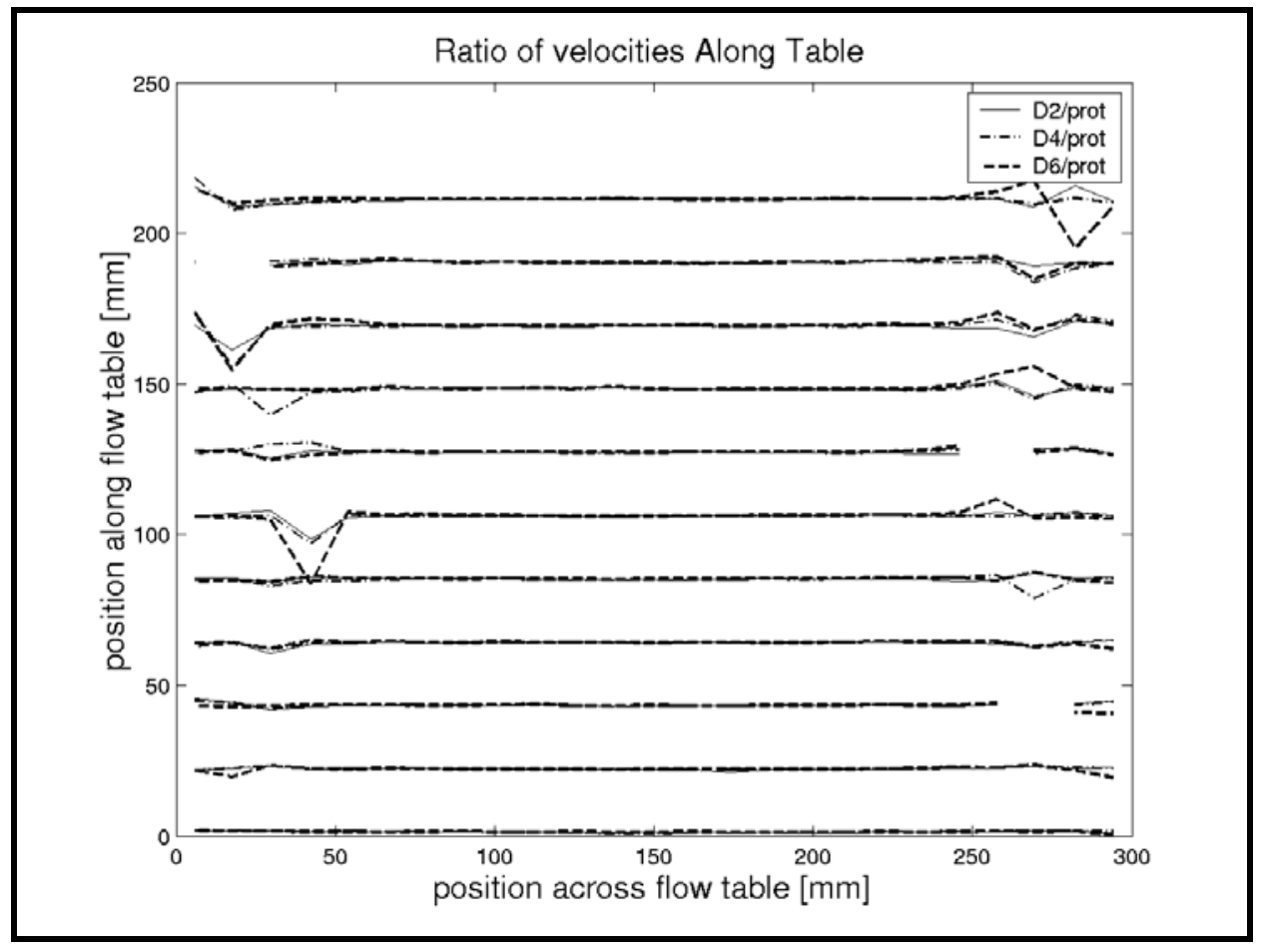

Figure B24. Case 2, $Q=1.0 \mathrm{~L} / \mathrm{sec}$, ratios between prototype and distortions 2, 4 , and 6 


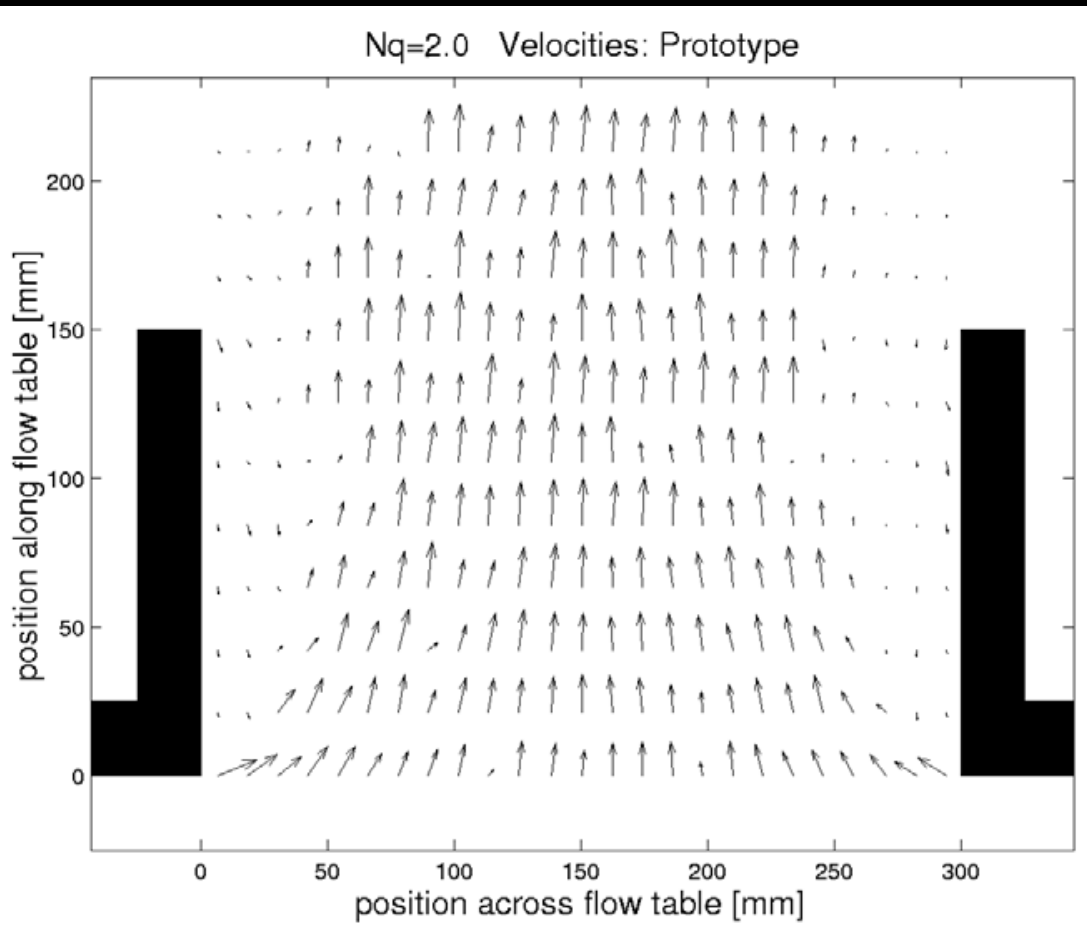

Figure B25. Case 2, $Q=0.75 \mathrm{~L} / \mathrm{sec}$, prototype

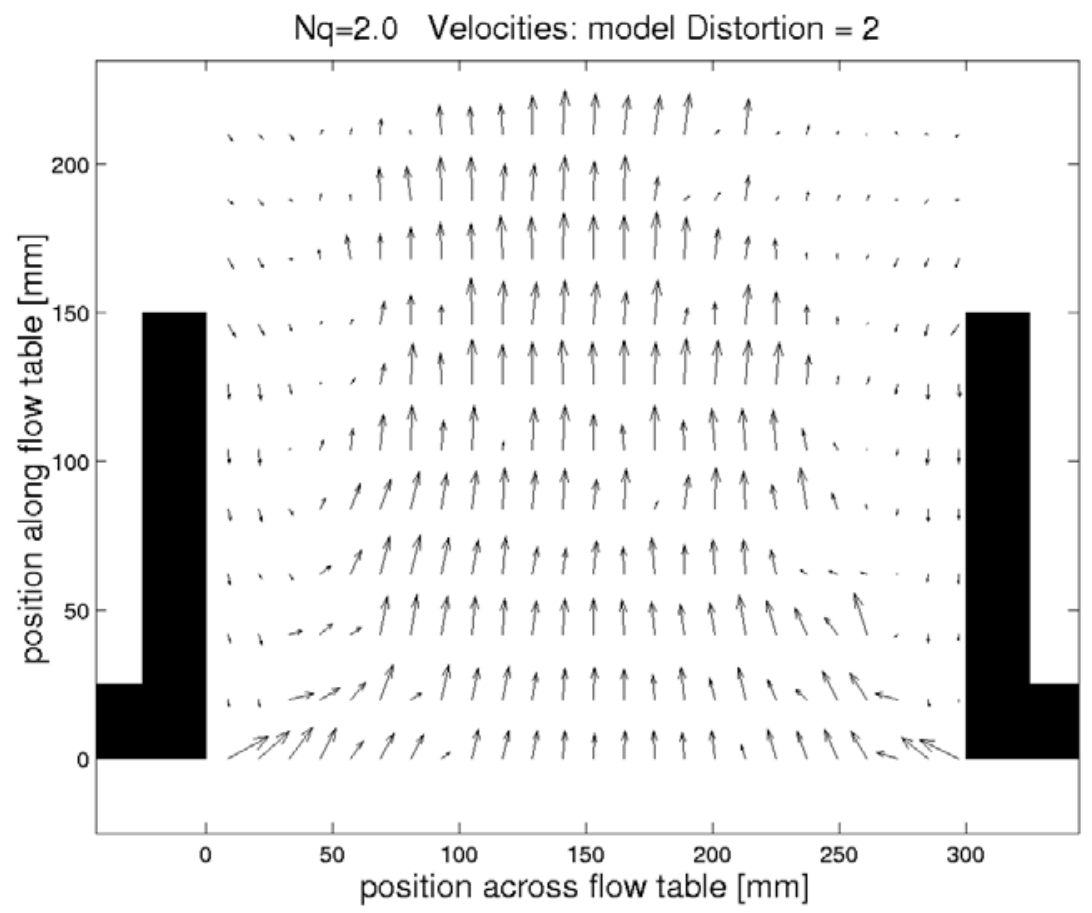

Figure B26. Case 2, $Q=0.75 \mathrm{~L} / \mathrm{sec}$, distortion $=2$ 


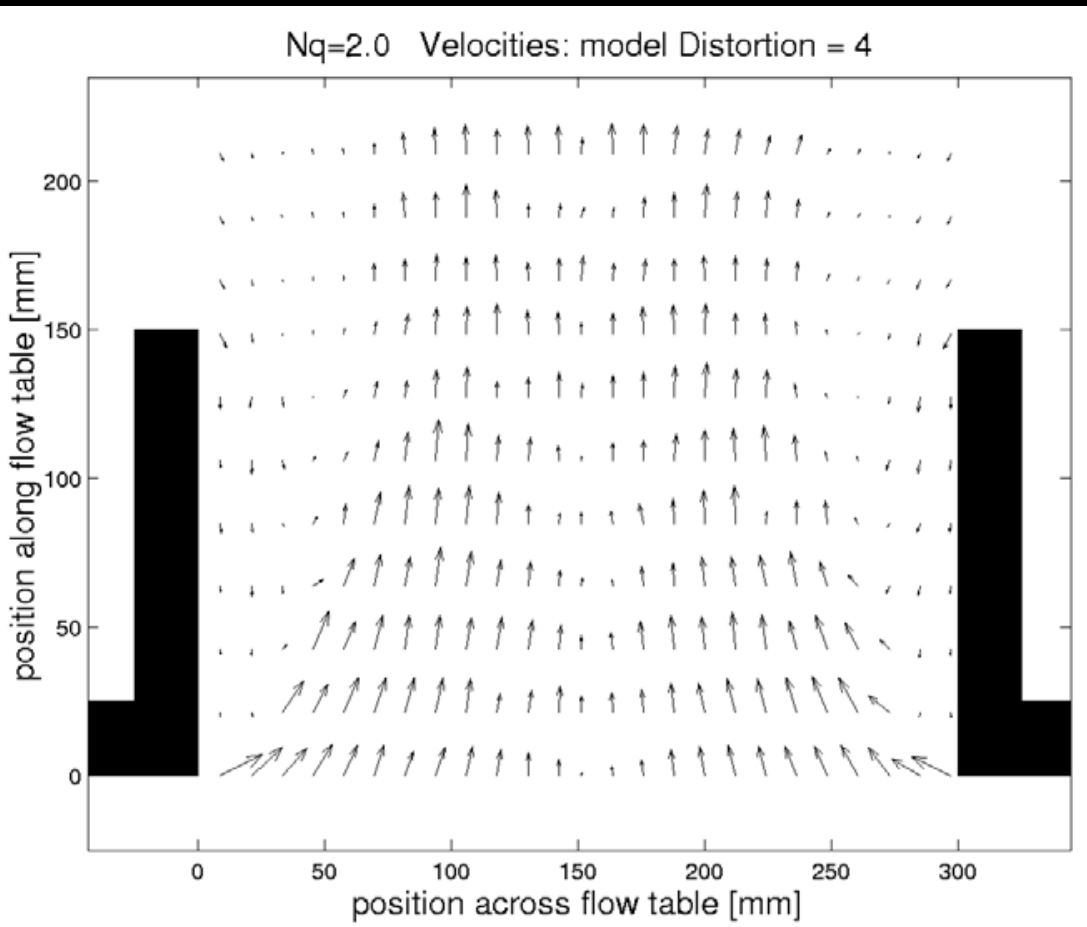

Figure B27. Case 2, $Q=0.75 \mathrm{~L} / \mathrm{sec}$, distortion $=4$

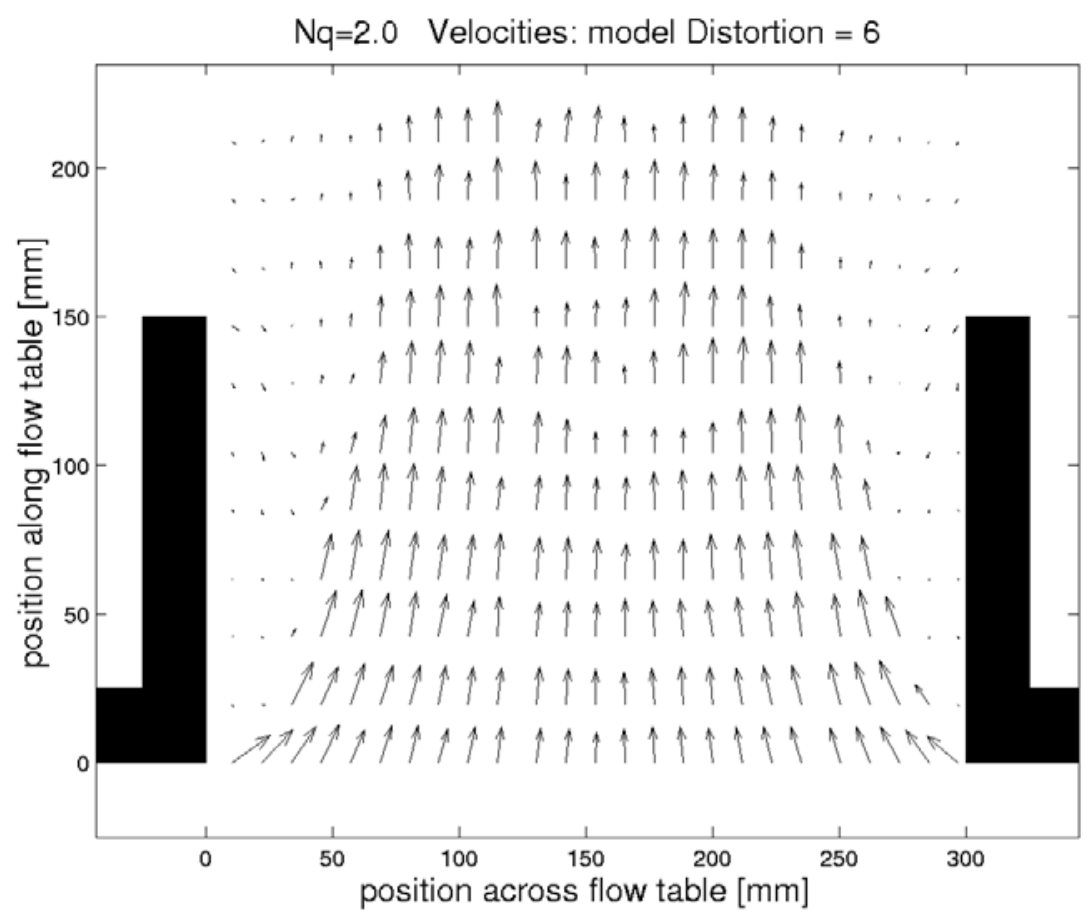

Figure B28. Case 2, $Q=0.75 \mathrm{~L} / \mathrm{sec}$, distortion $=6$ 


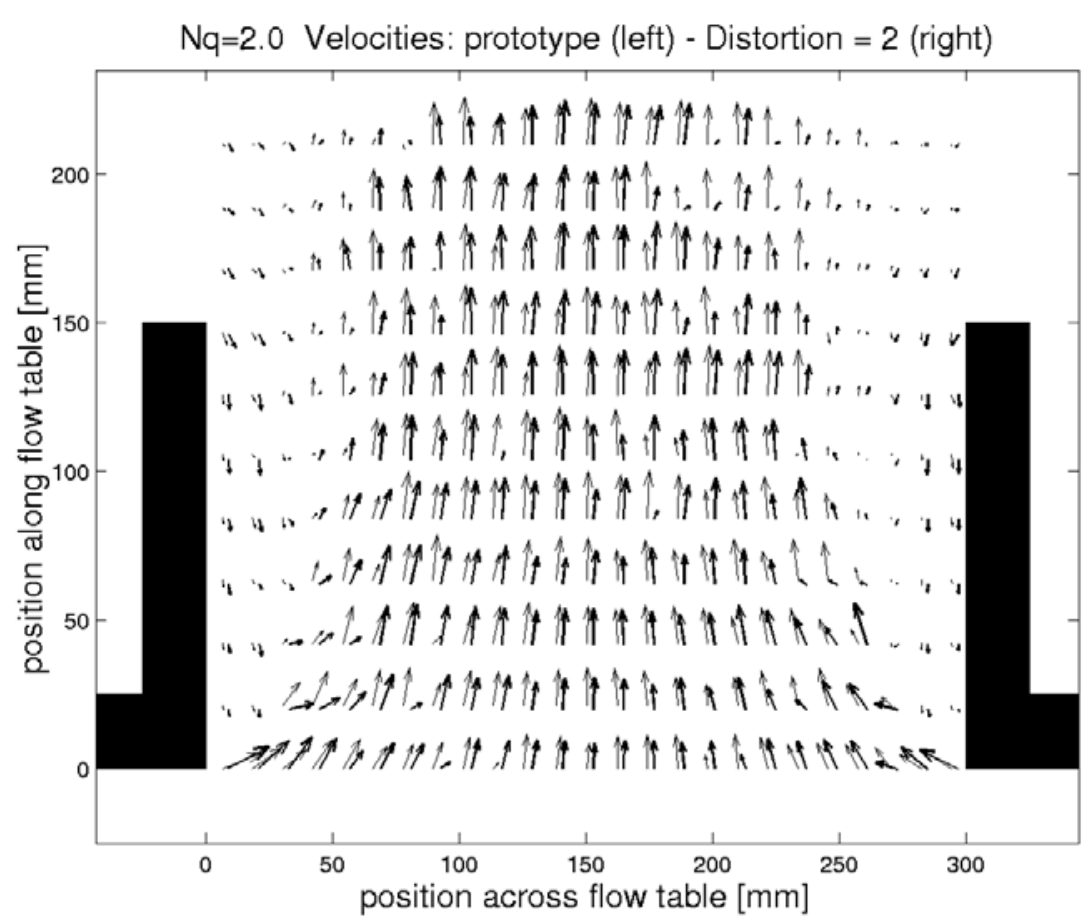

Figure B29. Case 2, $Q=0.75 \mathrm{~L} / \mathrm{sec}$, prototype vs. distortion $=2$

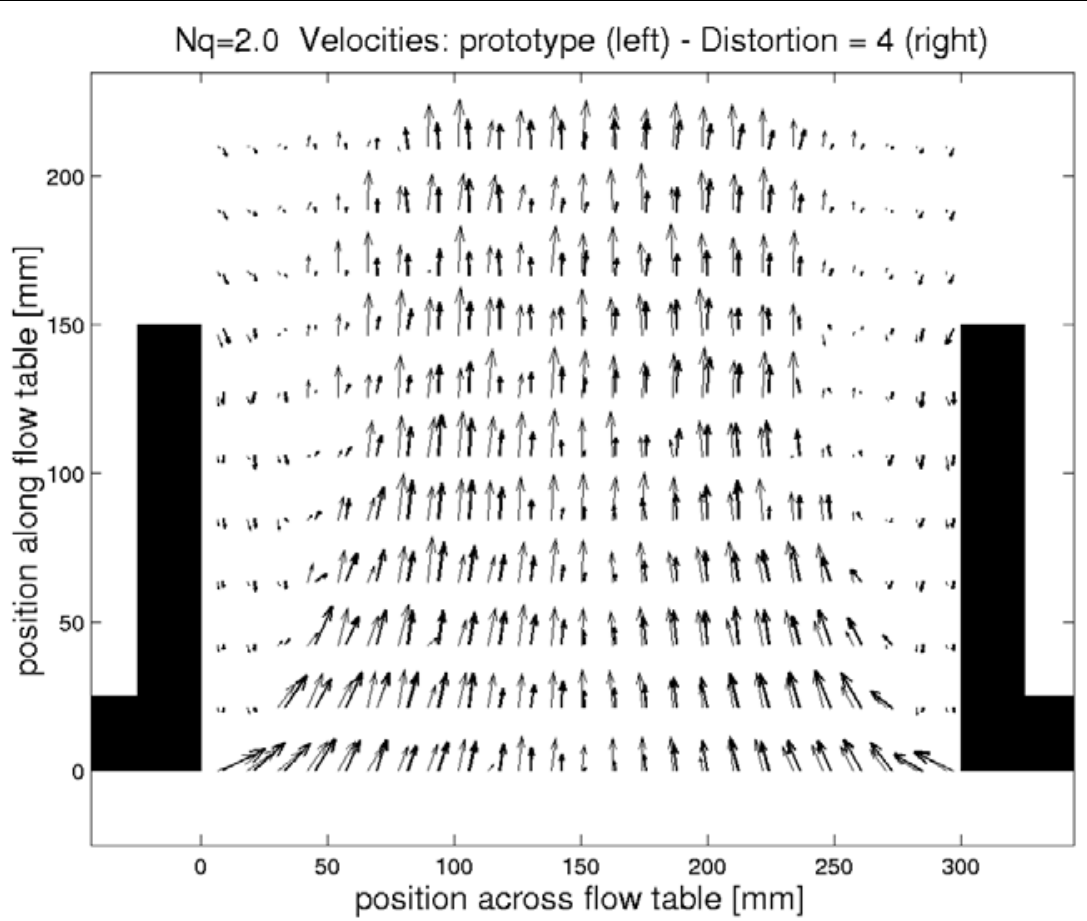

Figure B30. Case $2, Q=0.75 \mathrm{~L} / \mathrm{sec}$ prototype vs. distortion $=4$ 


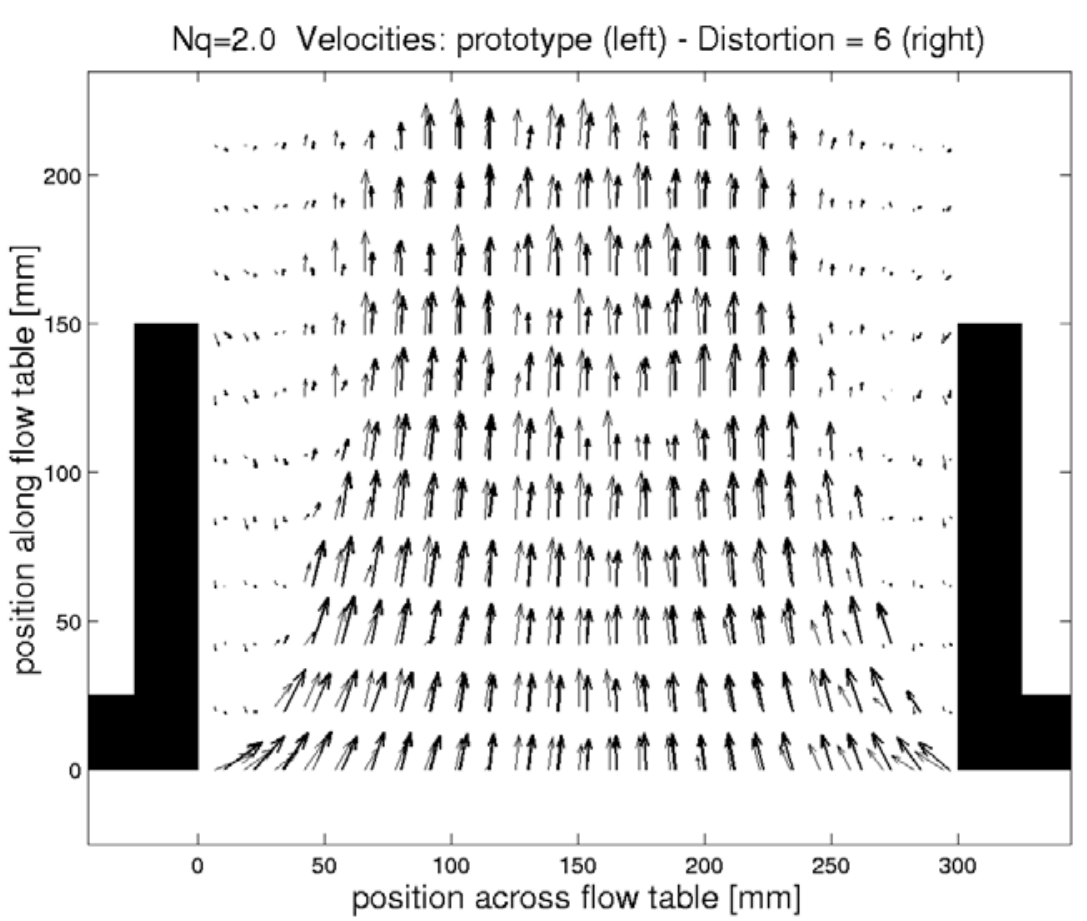

Figure B31. Case 2, $Q=0.75 \mathrm{~L} / \mathrm{sec}$, prototype vs. distortion $=6$

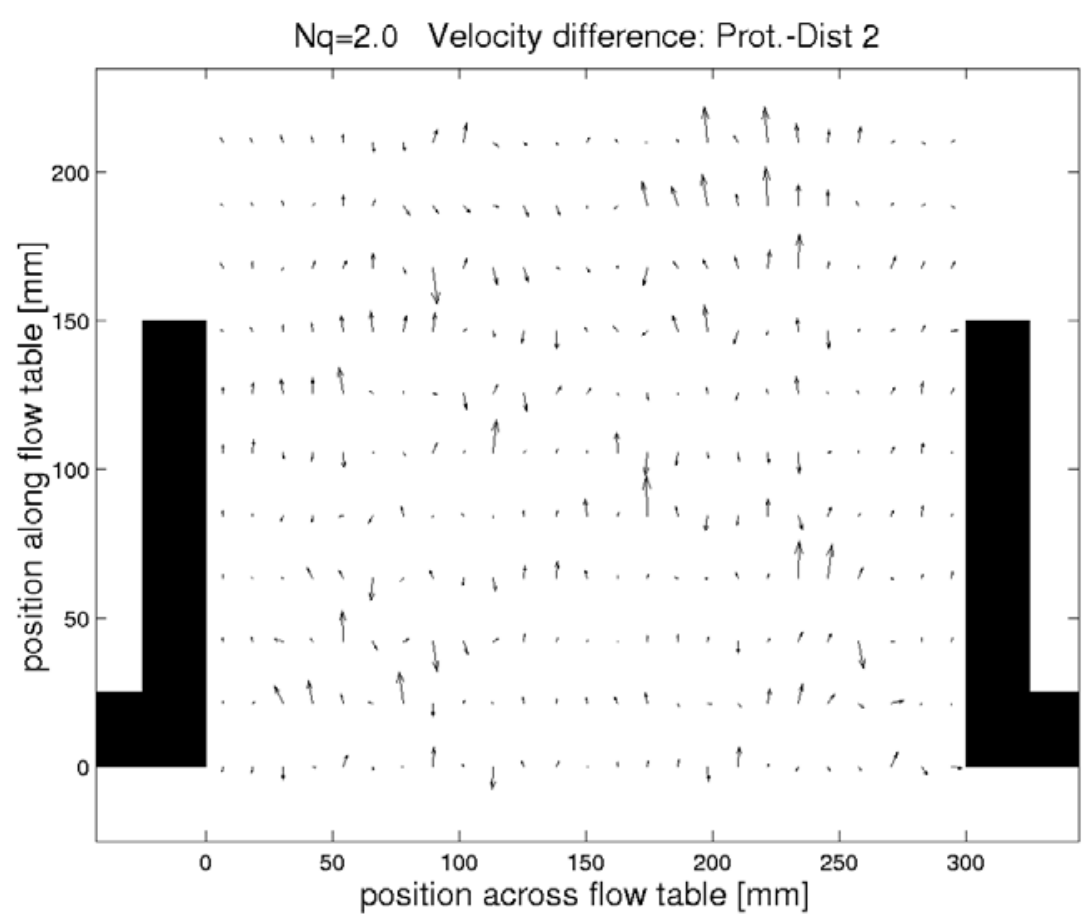

Figure B32. Case 2, $Q=0.75 \mathrm{~L} / \mathrm{sec}$, prototype minus distortion $=2$ 


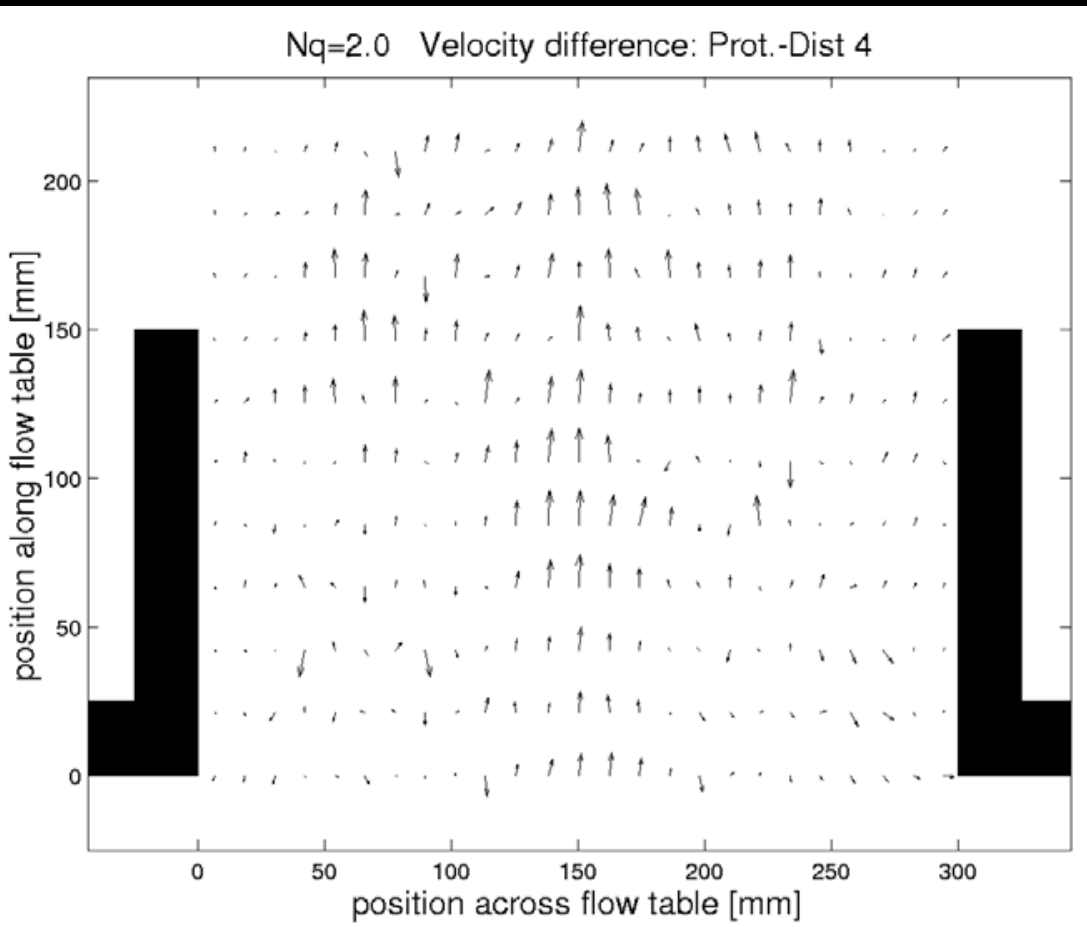

Figure B33. Case 2, $Q=0.75 \mathrm{~L} / \mathrm{sec}$, prototype minus distortion $=4$

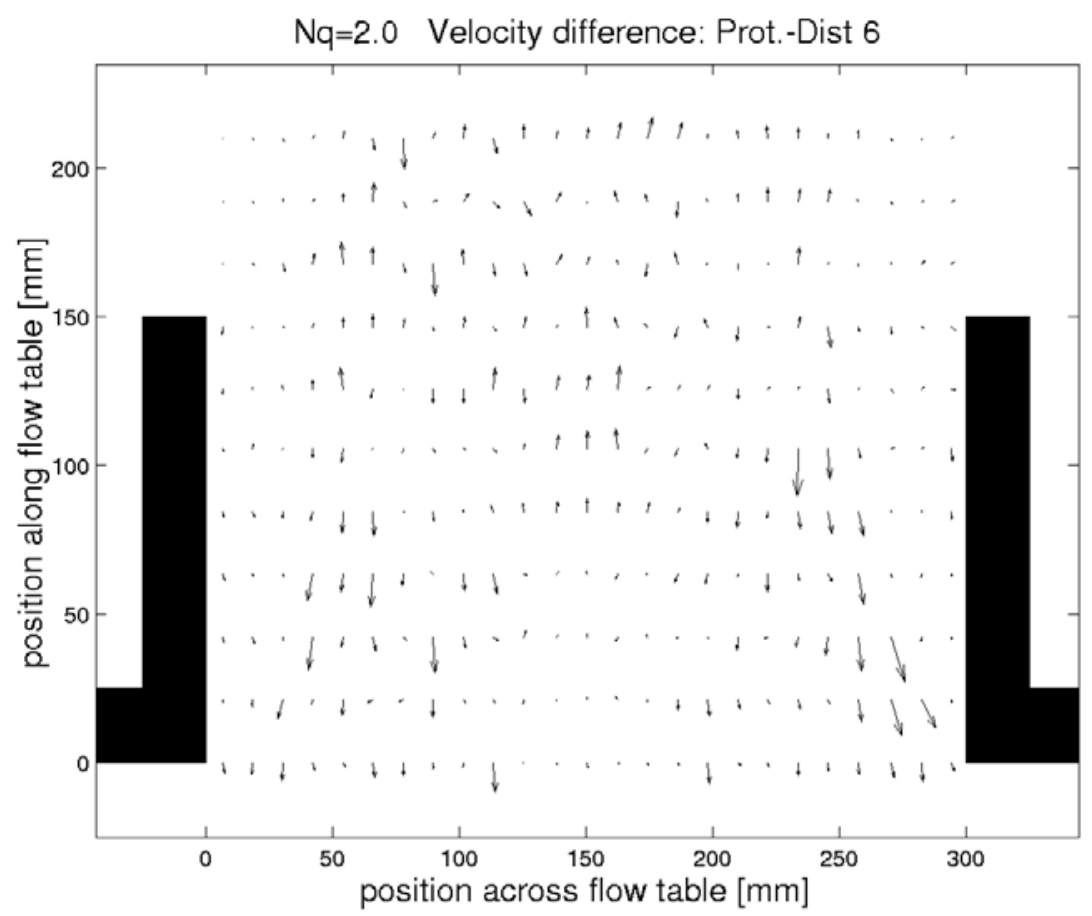

Figure B34. Case 2, $Q=0.75 \mathrm{~L} / \mathrm{sec}$, prototype minus distortion $=6$ 


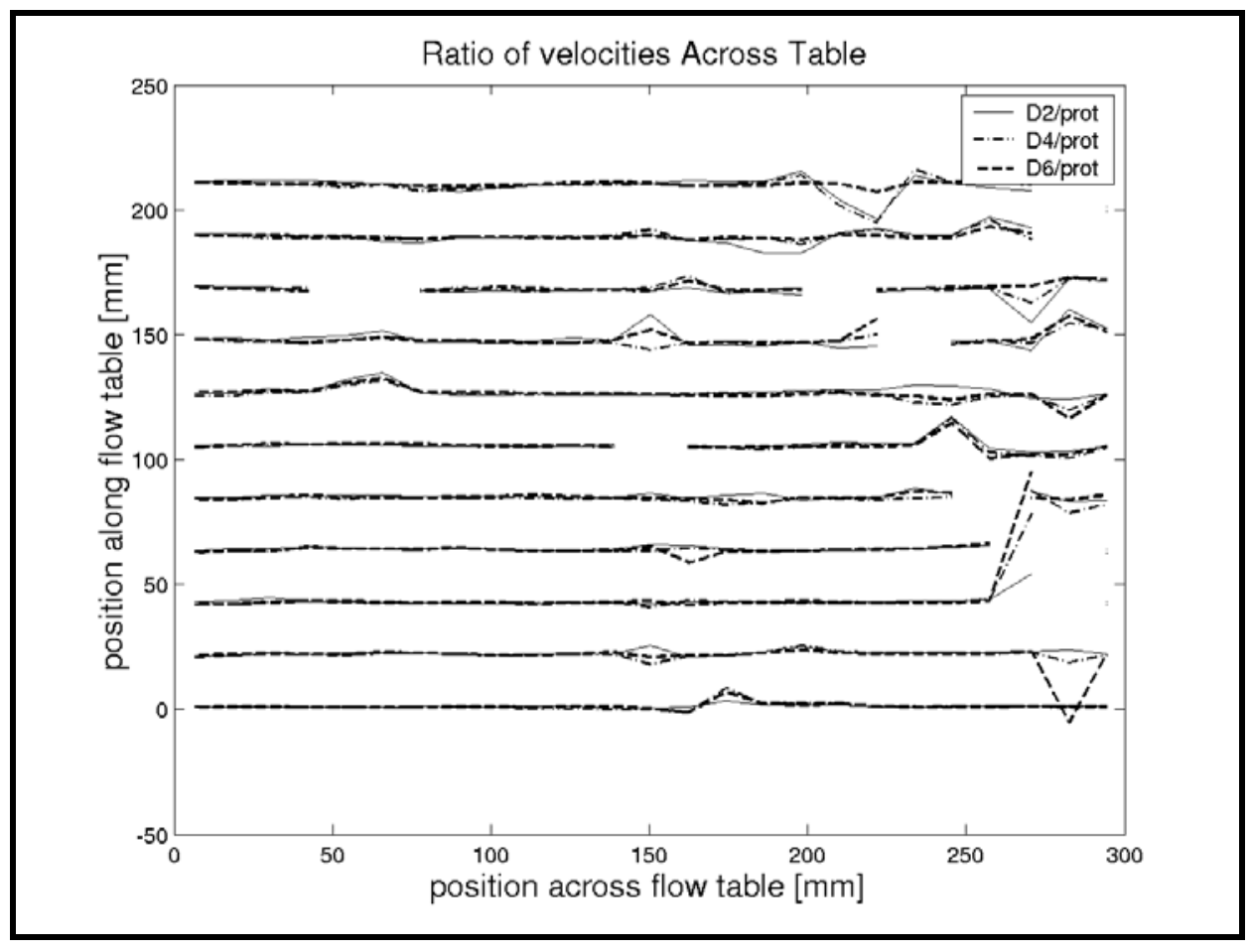

Figure B35. Case 2, $Q=0.75 \mathrm{~L} / \mathrm{sec}$, ratios between prototype and distortions 2, 4 , and 6

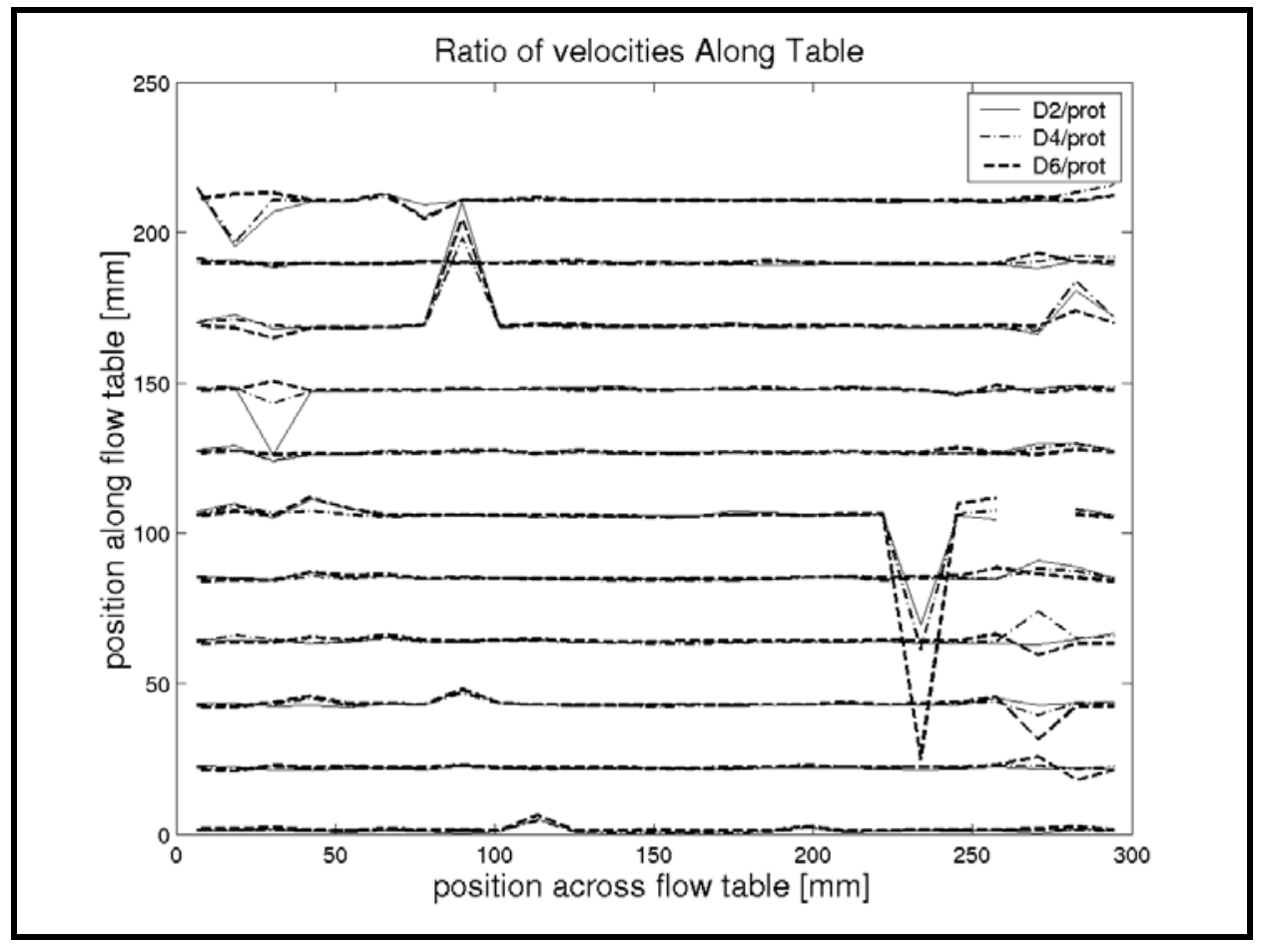

Figure B36. Case 2, $Q=0.75 \mathrm{~L} / \mathrm{sec}$, ratios between prototype and distortions 2, 4 , and 6 


\section{Appendix C \\ Case 3 - Flow Separation at Sloping Edge}

This appendix contains the complete set of comparisons between prototype and geometrically-distorted models for the case of flow separation on the boundary of a free jet created by a gap with sloping edges. See Chapter 5 for a description of the experiments and discussion of results. 


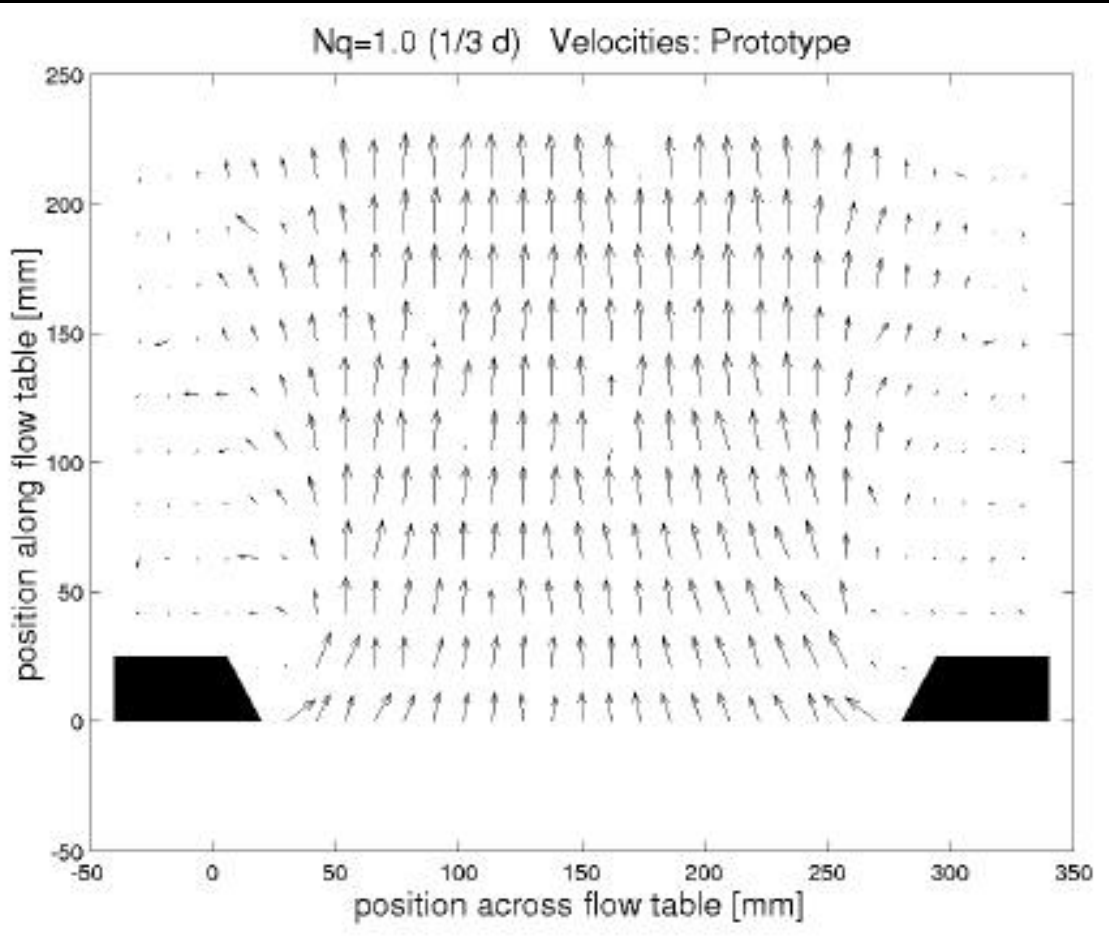

Figure C1. Case $3(1 / 3 d), Q=1.5 \mathrm{~L} / \mathrm{sec}$, prototype

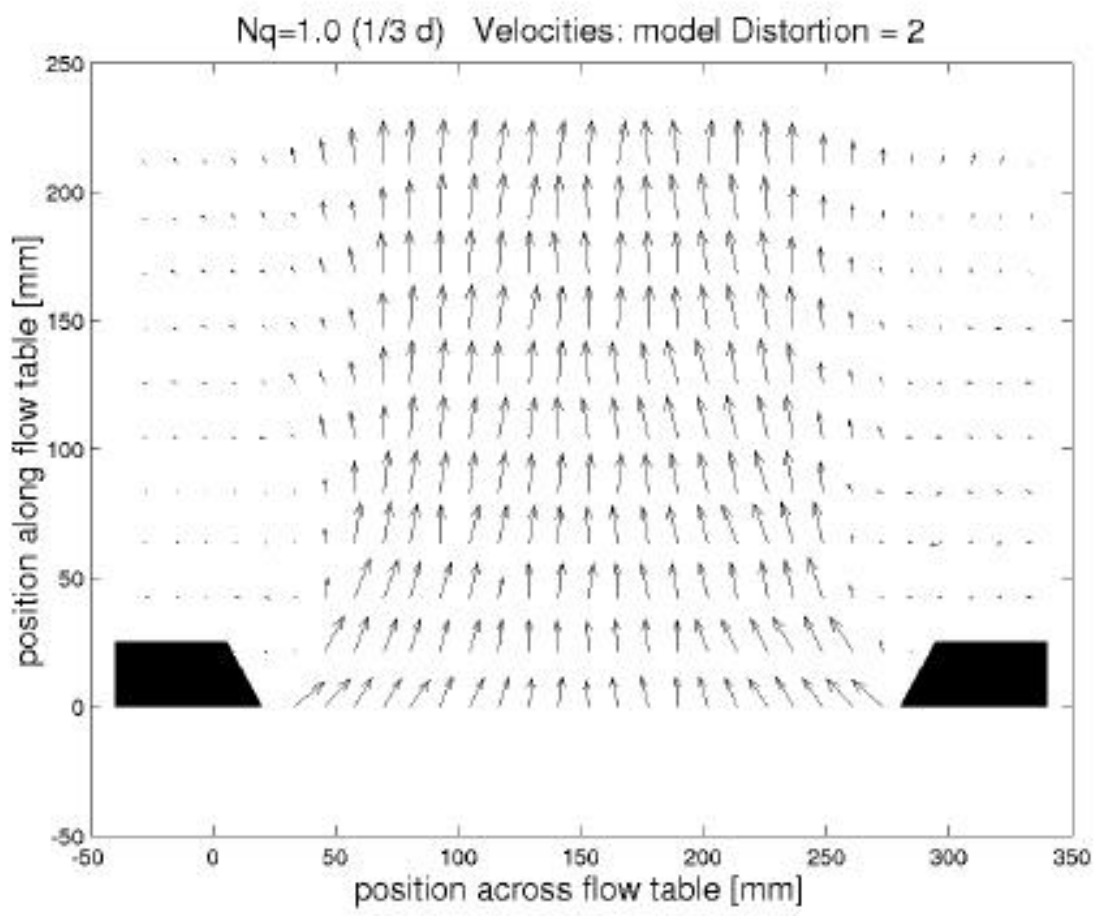

Figure C2. Case $3(1 / 3 d), Q=1.5 \mathrm{~L} / \mathrm{sec}$, distortion $=2$ 


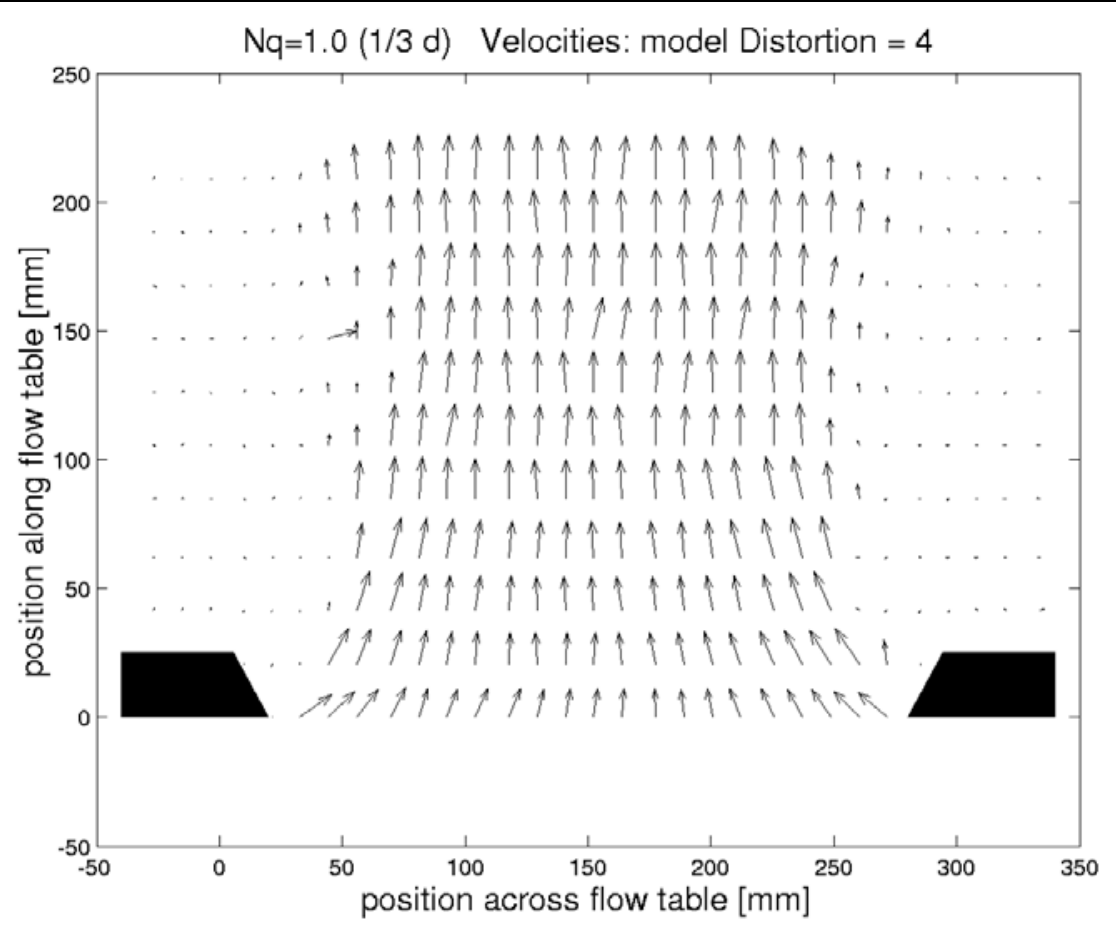

Figure C3. Case $3(1 / 3 d), Q=1.5 \mathrm{~L} / \mathrm{sec}$, distortion $=4$

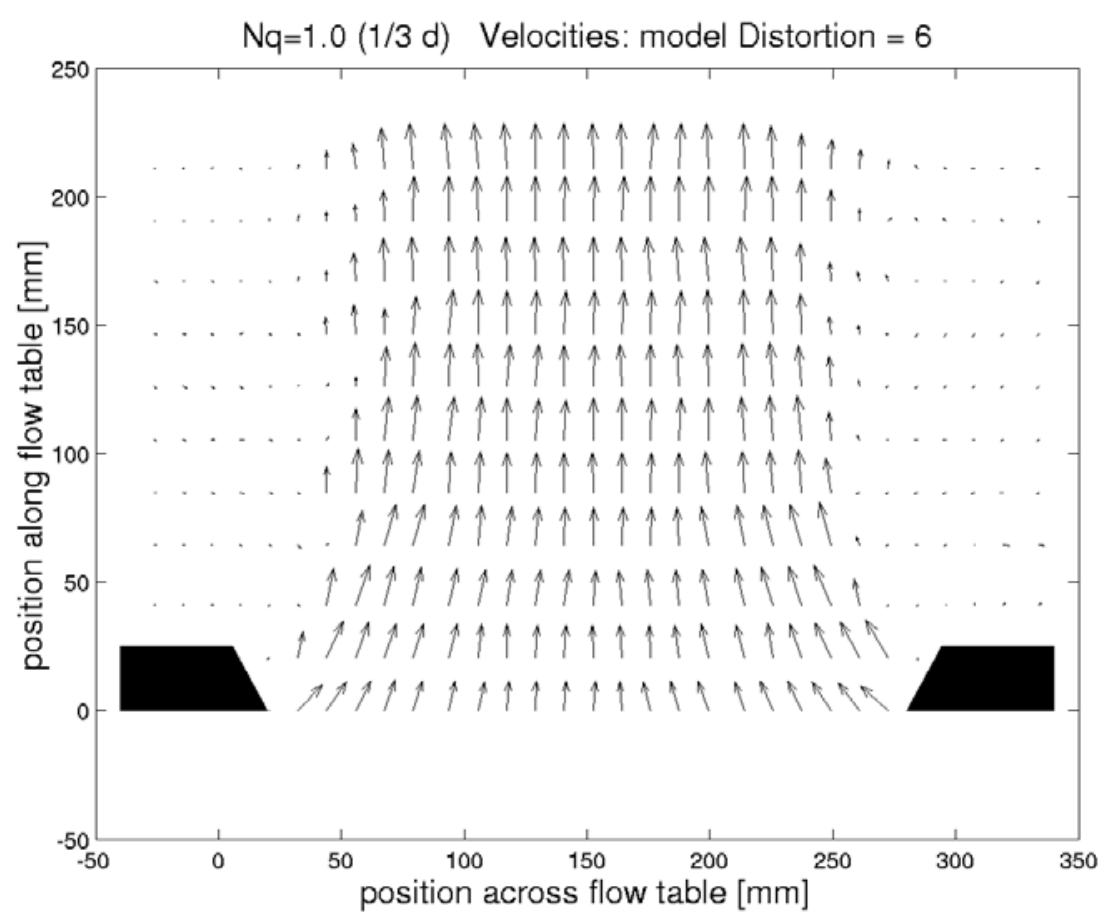

Figure C4. Case $3(1 / 3 d), Q=1.5 \mathrm{~L} / \mathrm{sec}$, distortion $=6$ 


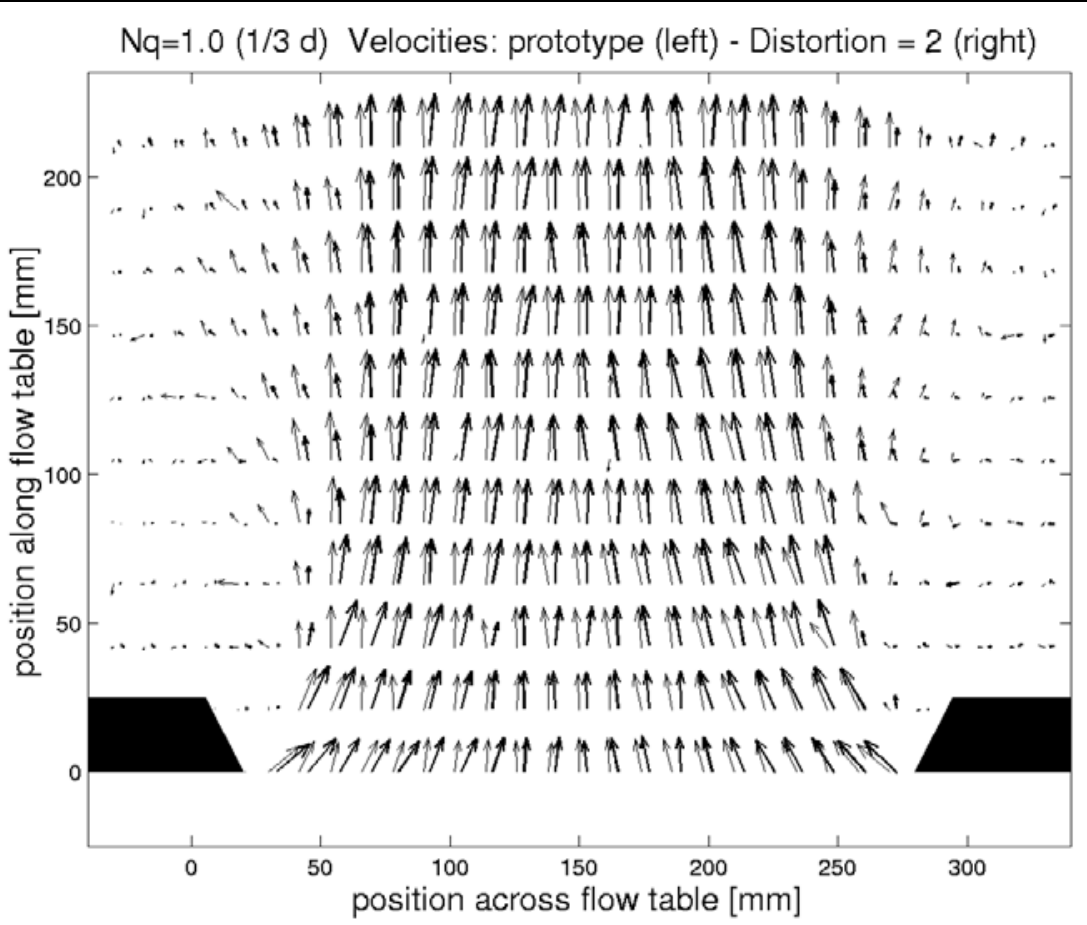

Figure C5. Case $3(1 / 3 d), Q=1.5 \mathrm{~L} / \mathrm{sec}$, prototype vs. distortion = 2

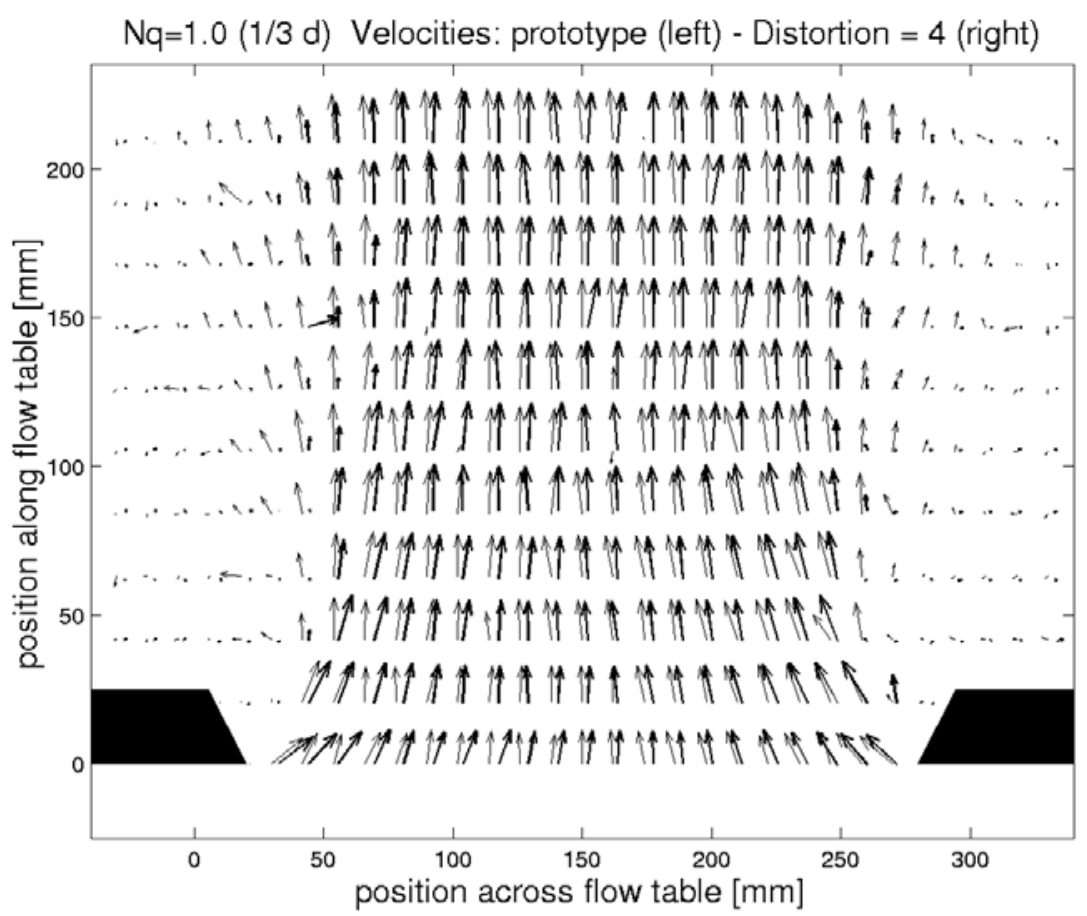

Figure C6. Case $3(1 / 3 d), Q=1.5 \mathrm{~L} / \mathrm{sec}$, prototype vs. distortion = 4 


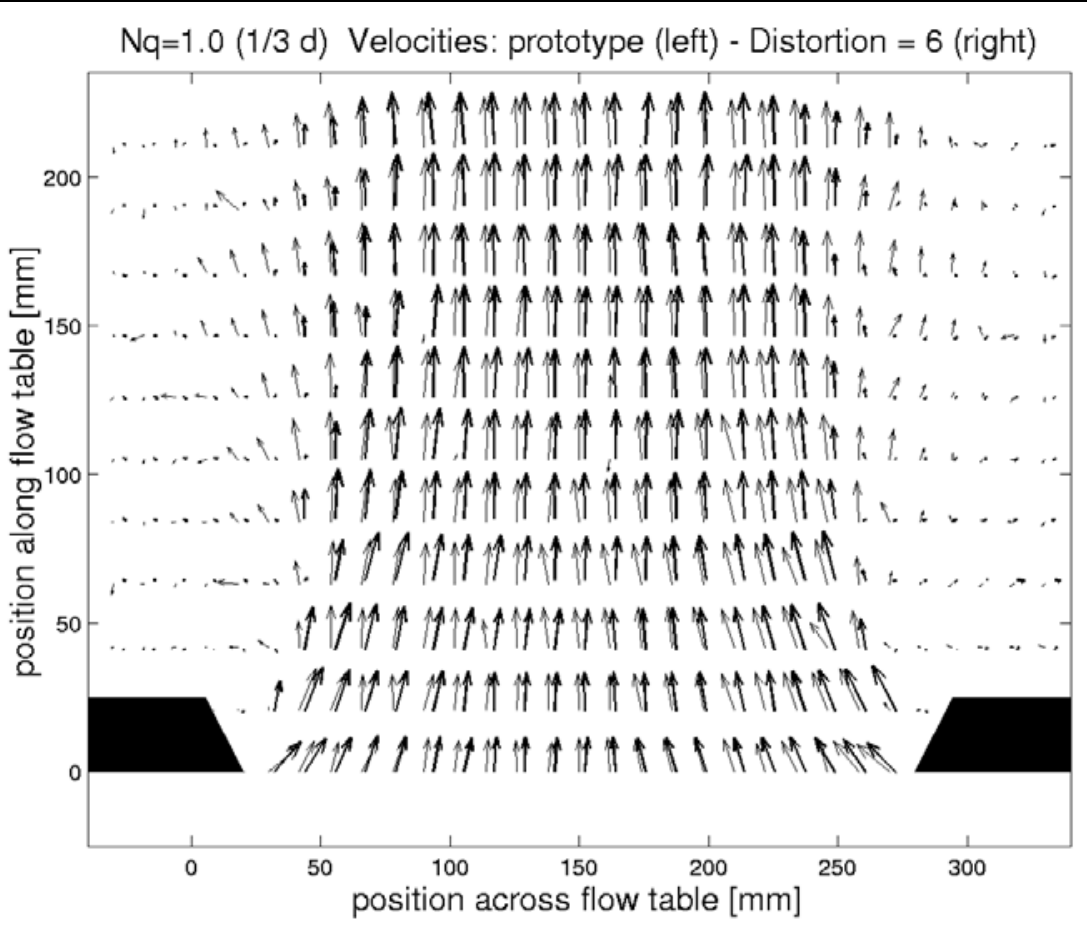

Figure C7. Case $3(1 / 3 d), Q=1.5 \mathrm{~L} / \mathrm{sec}$, prototype vs. distortion $=6$

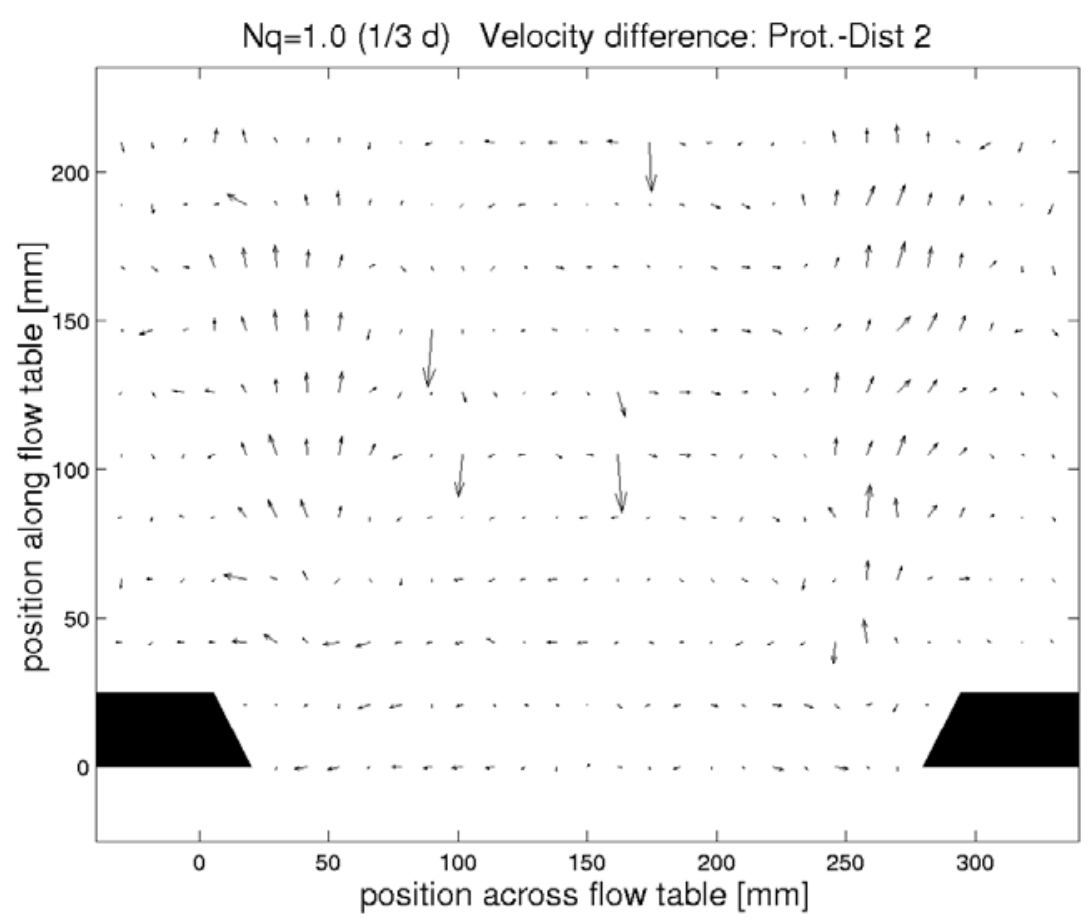

Figure C8. Case $3(1 / 3 d), Q=1.5 \mathrm{~L} / \mathrm{sec}$, prototype minus distortion = 2 


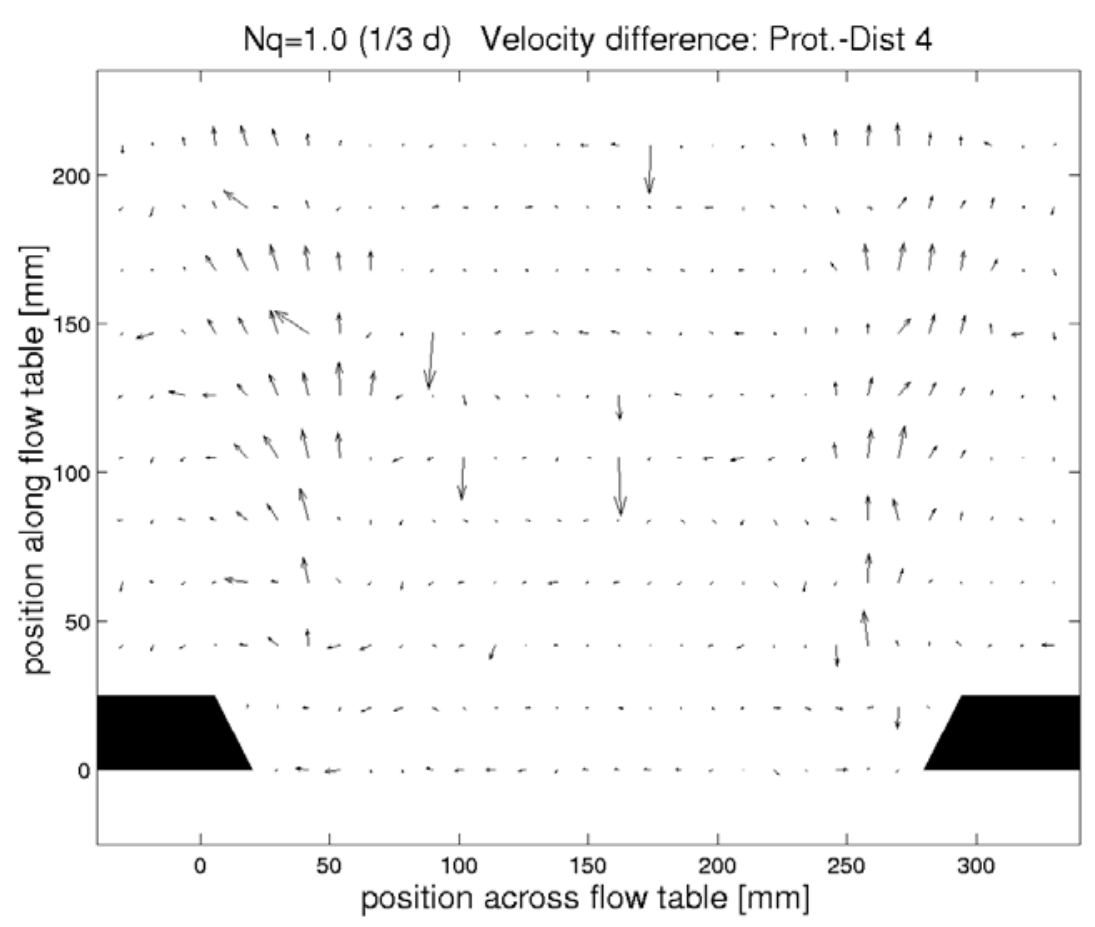

Figure C9. Case $3(1 / 3 d), Q=1.5 \mathrm{~L} / \mathrm{sec}$, prototype minus distortion $=4$

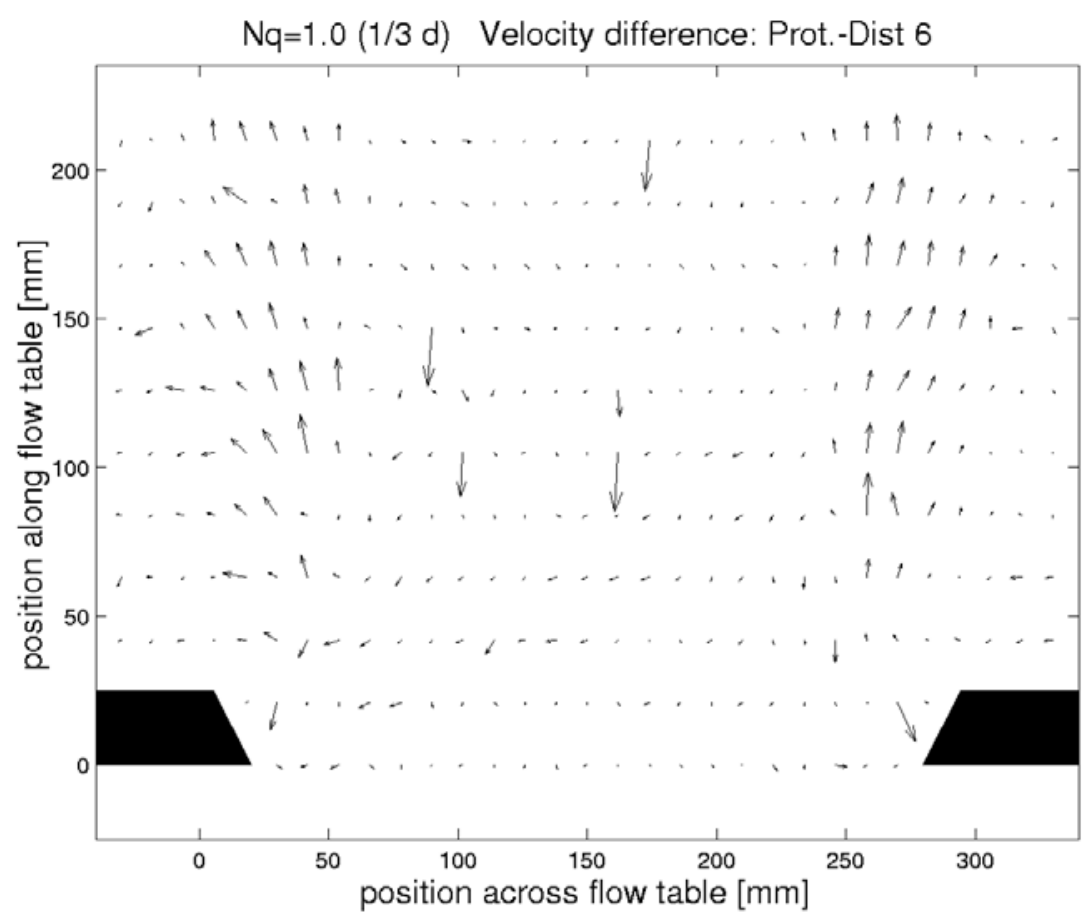

Figure C10. Case $3(1 / 3 d), Q=1.5 \mathrm{~L} / \mathrm{sec}$, prototype minus distortion $=6$ 


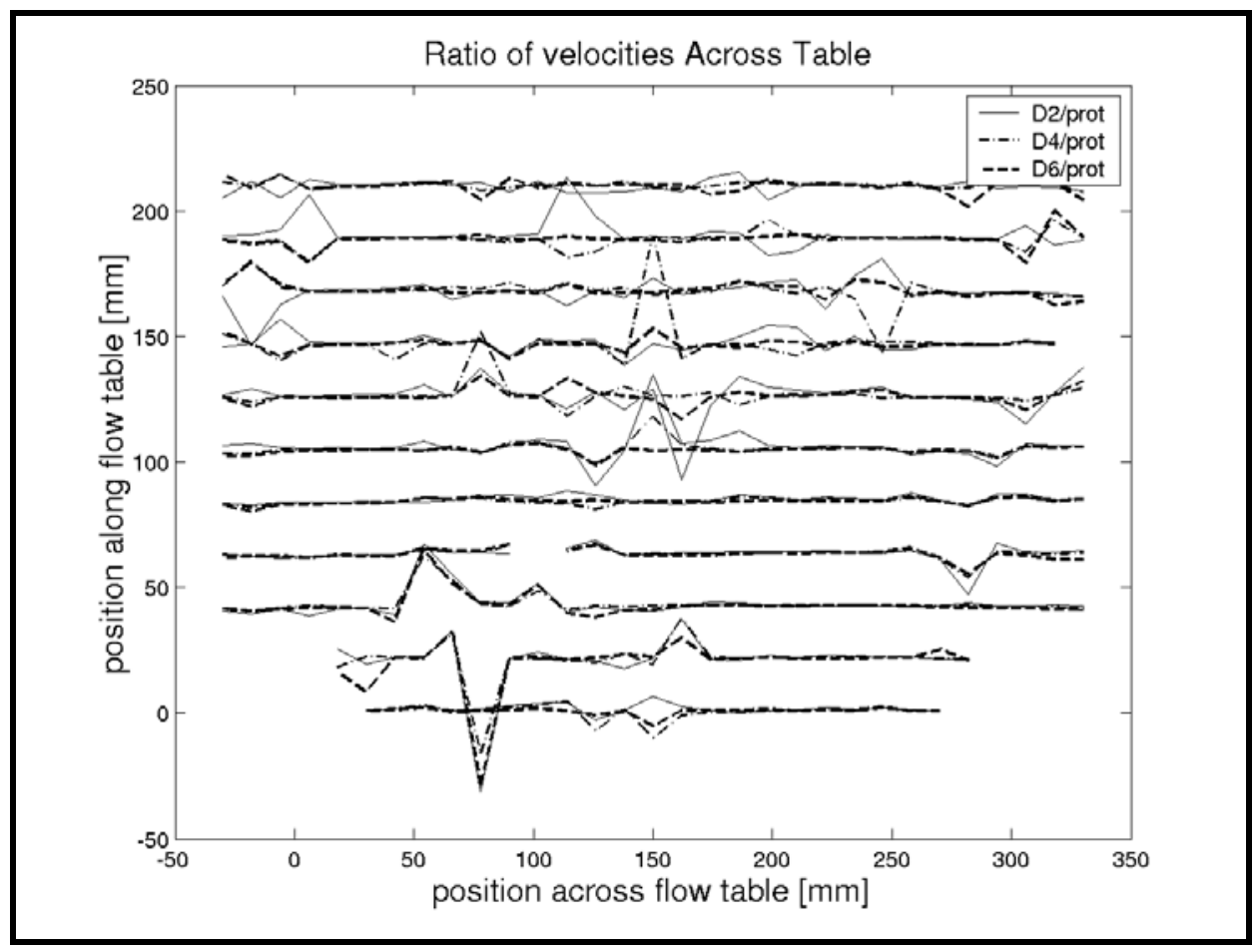

Figure $\mathrm{C} 11$. Case $3(1 / 3 d), Q=1.5 \mathrm{~L} / \mathrm{sec}$, ratios between prototype and distortions 2,4 , and 6

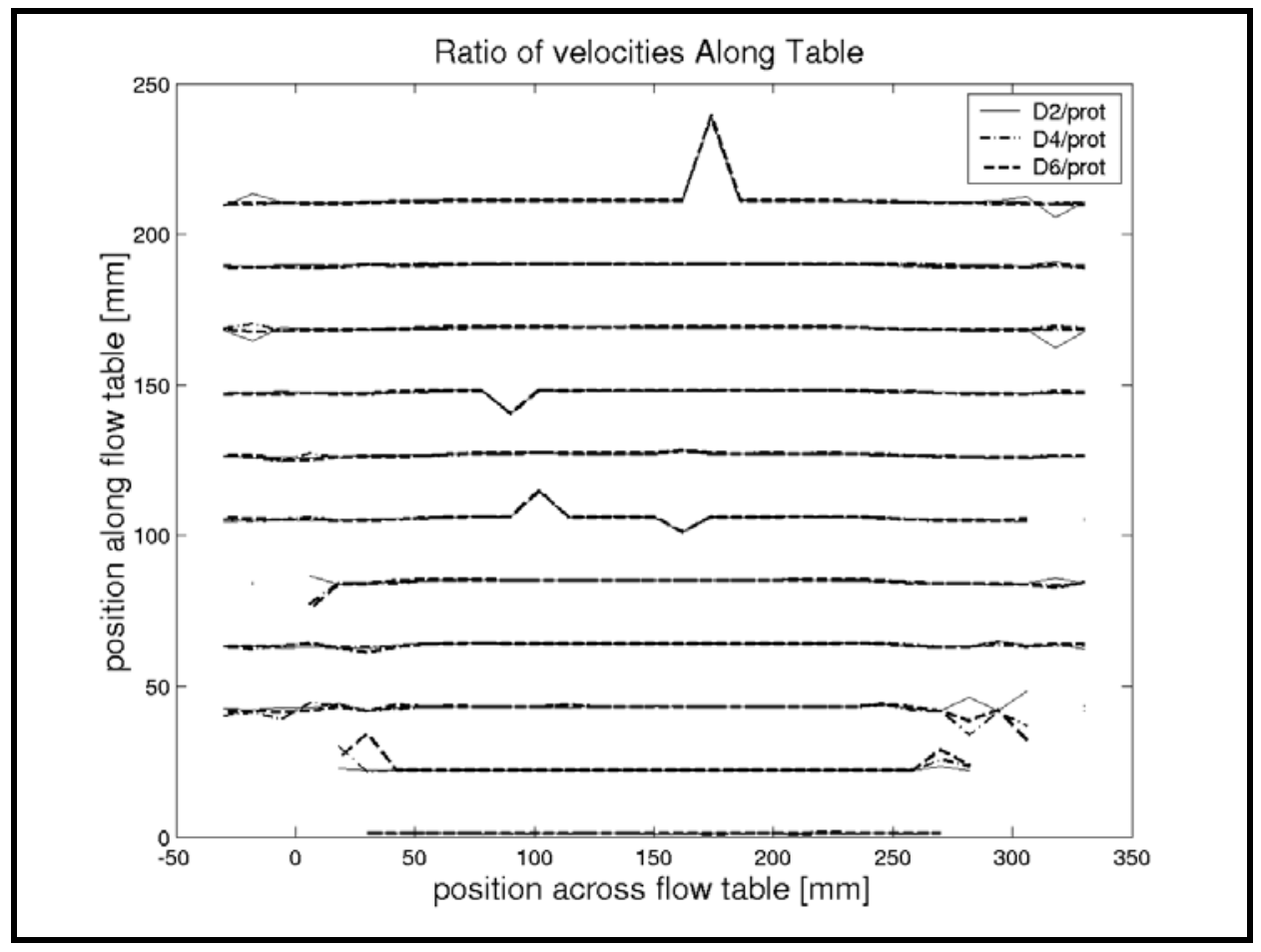

Figure $\mathrm{C} 12$. Case $3(1 / 3 d), Q=1.5 \mathrm{~L} / \mathrm{sec}$, ratios between prototype and distortions 2,4 , and 6 


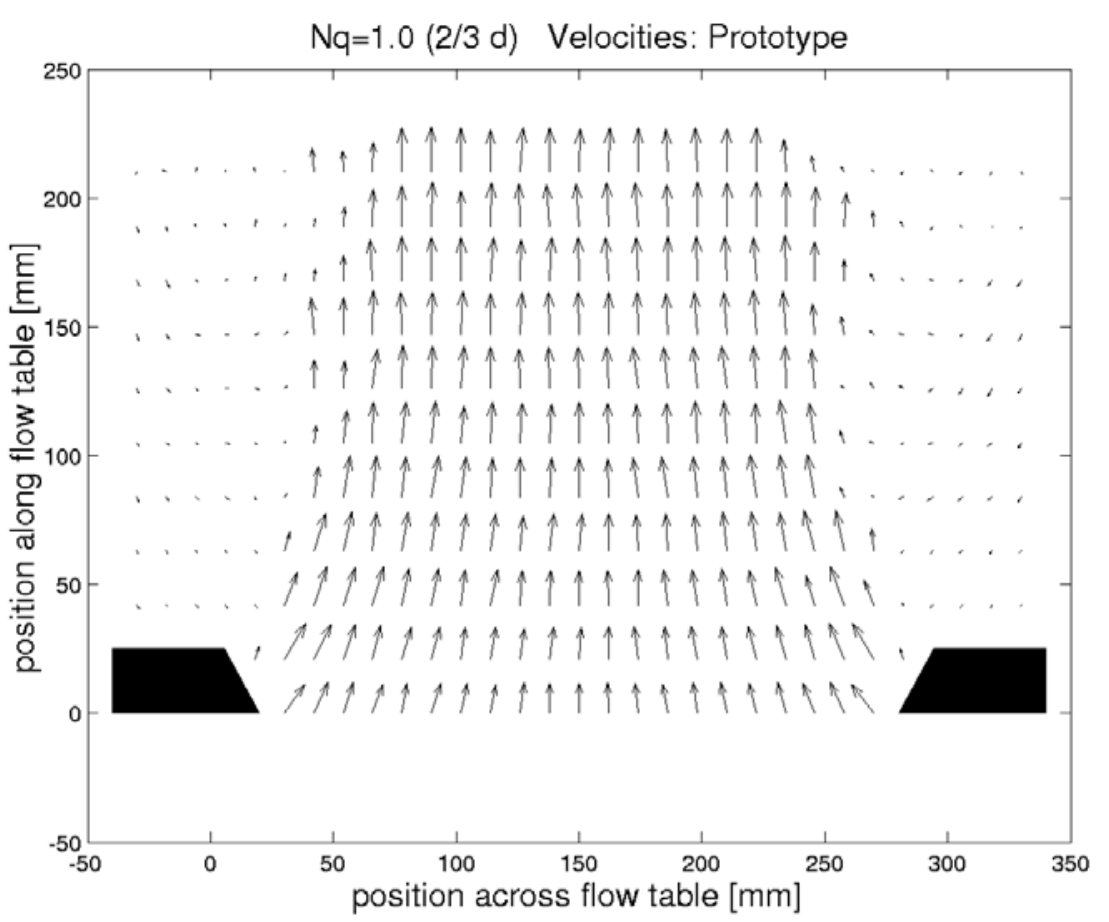

Figure C13. Case $3(2 / 3 d), Q=1.5 \mathrm{~L} / \mathrm{sec}$, prototype

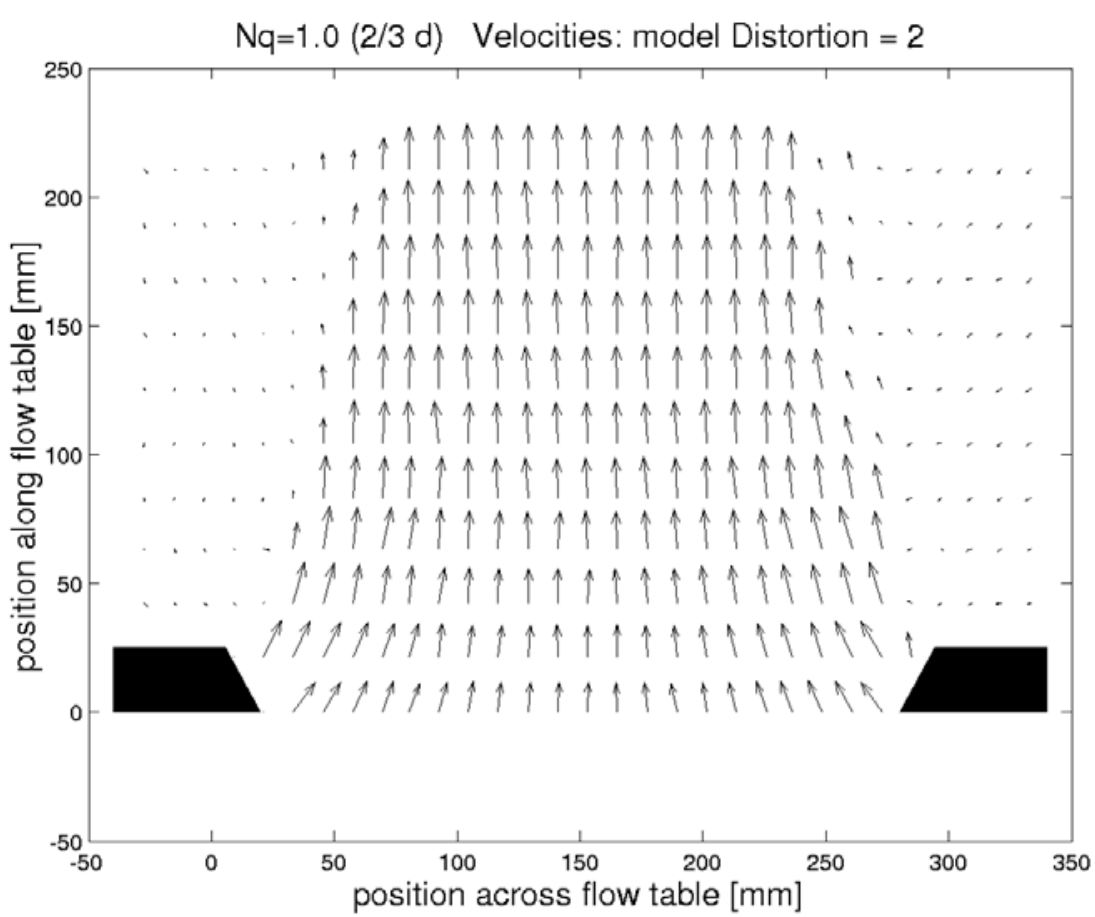

Figure C14. Case $3(2 / 3 d), Q=1.5 \mathrm{~L} / \mathrm{sec}$, distortion $=2$ 


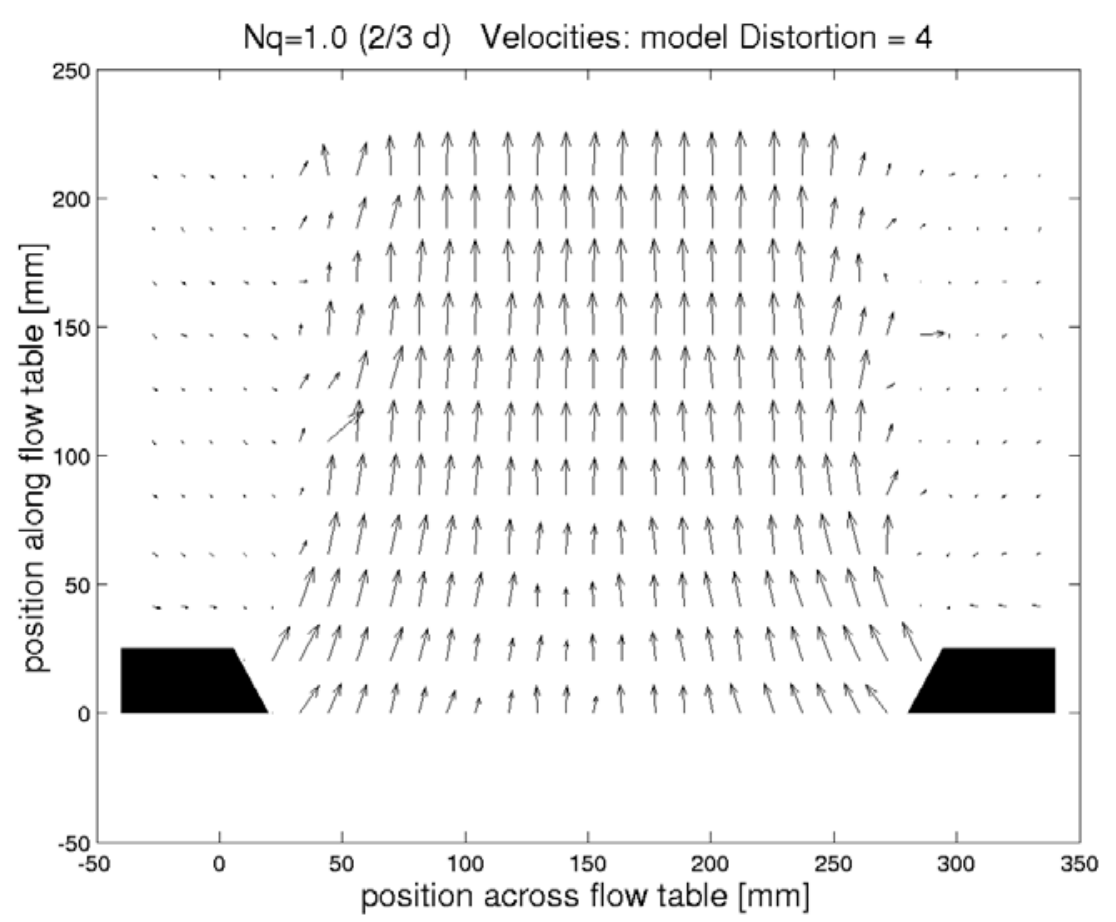

Figure C15. Case $3(2 / 3 d), Q=1.5 \mathrm{~L} / \mathrm{sec}$, distortion $=4$

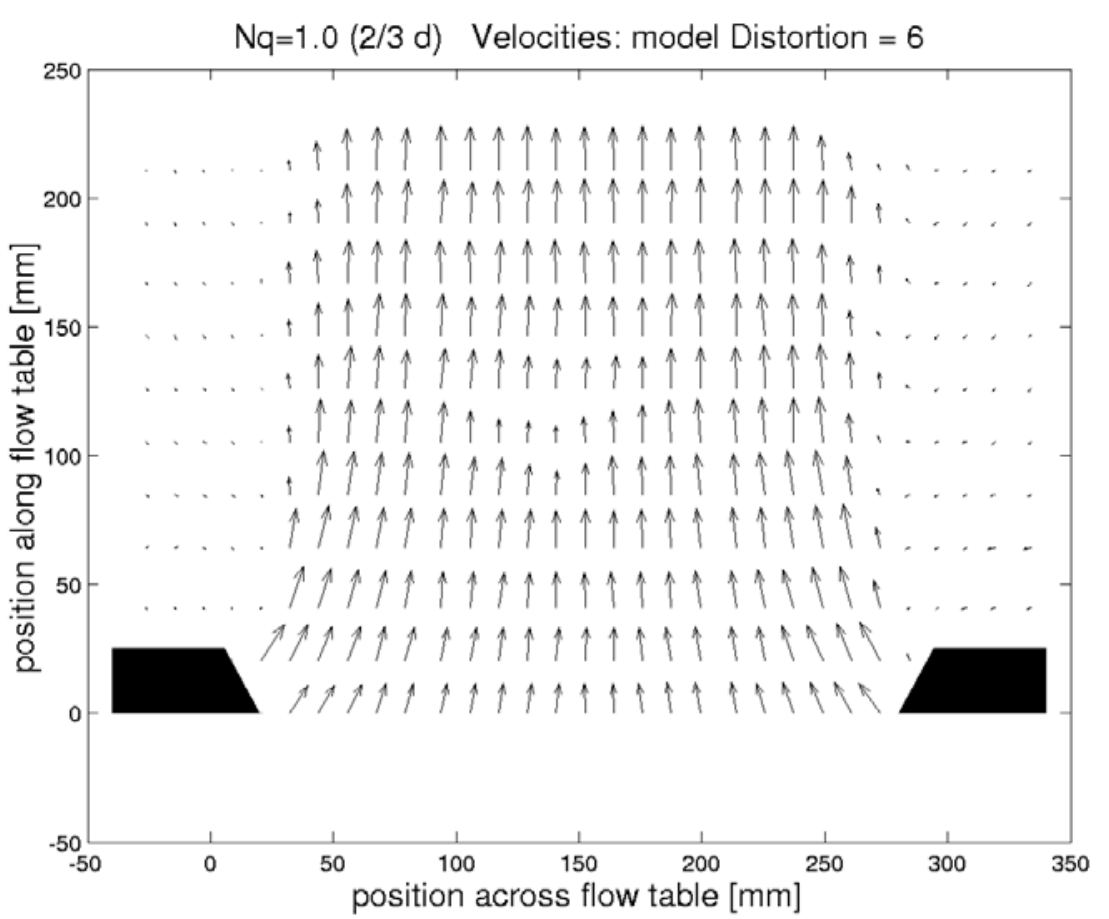

Figure C16. Case $3(2 / 3 d), Q=1.5 \mathrm{~L} / \mathrm{sec}$, distortion $=6$ 


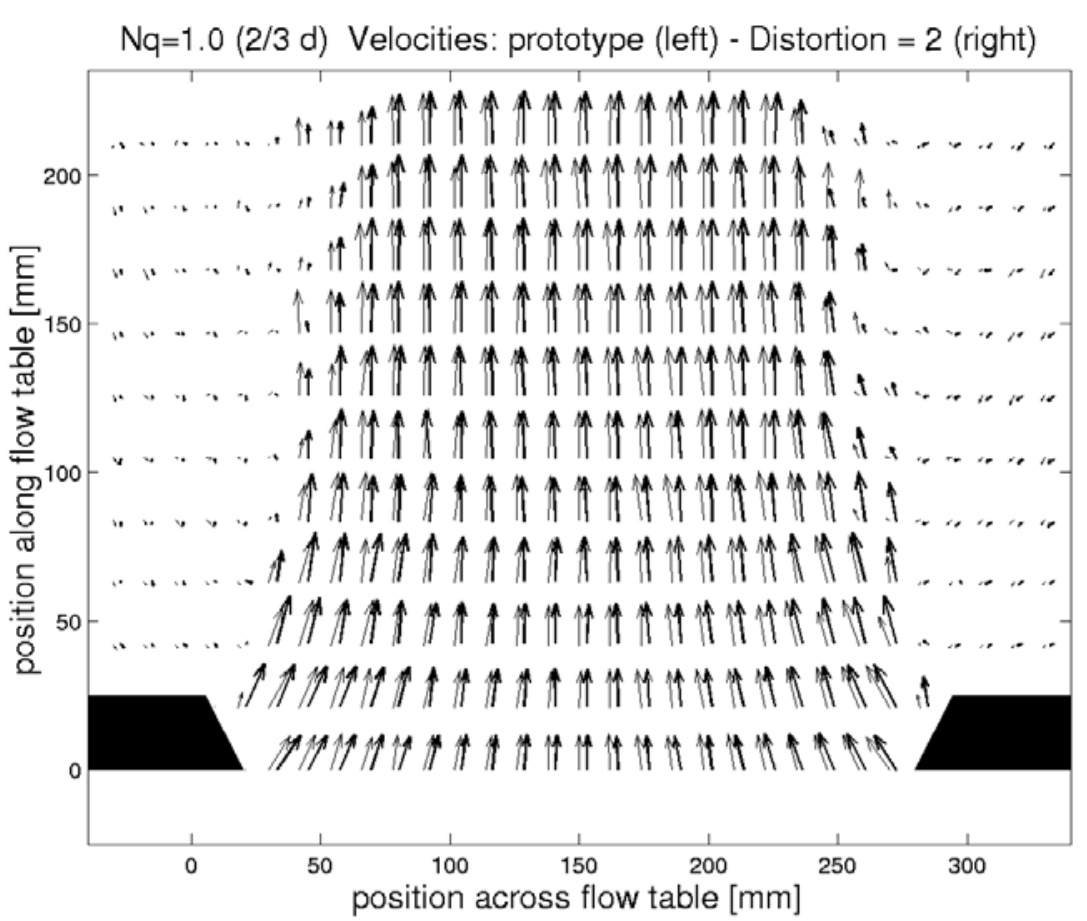

Figure C17. Case $3(2 / 3 d), Q=1.5 \mathrm{~L} / \mathrm{sec}$, prototype vs. distortion $=2$

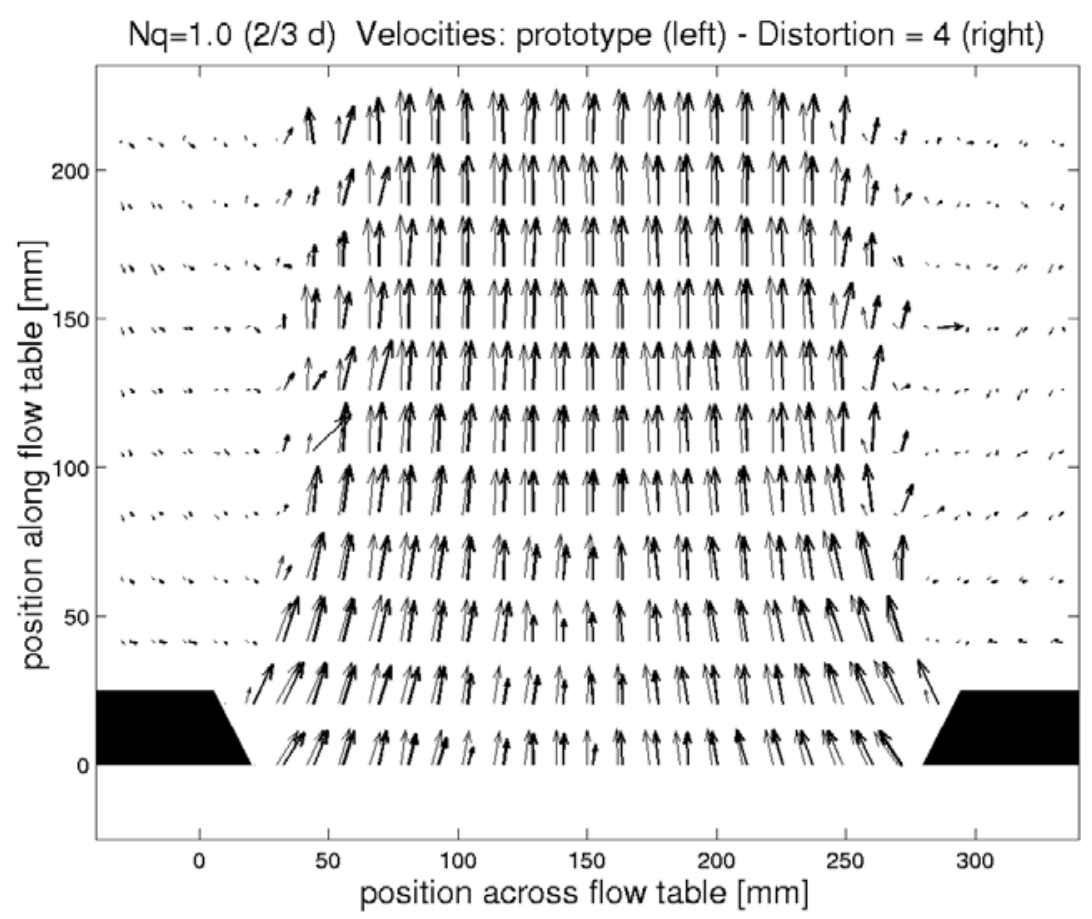

Figure C18. Case $3(2 / 3 d), Q=1.5 \mathrm{~L} / \mathrm{sec}$, prototype vs. distortion $=4$ 


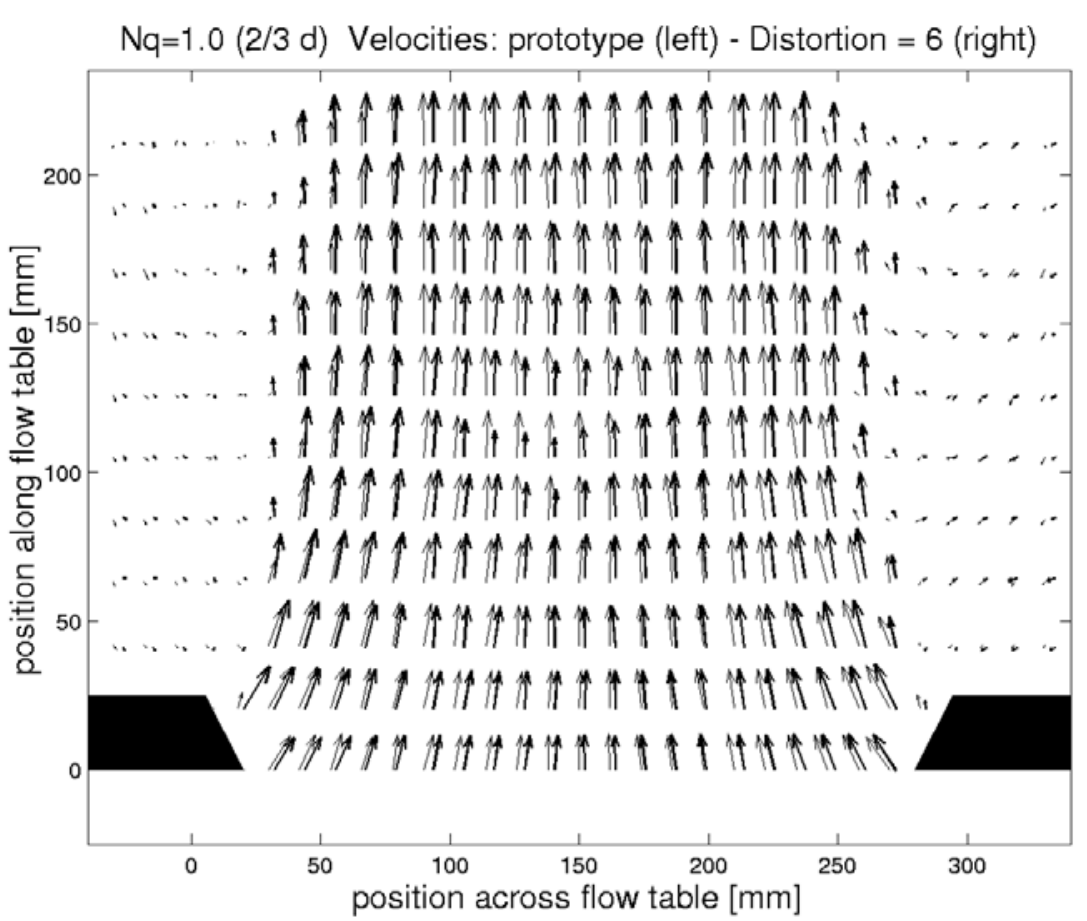

Figure C19. Case $3(2 / 3 d), Q=1.5 \mathrm{~L} / \mathrm{sec}$, prototype vs. distortion $=6$

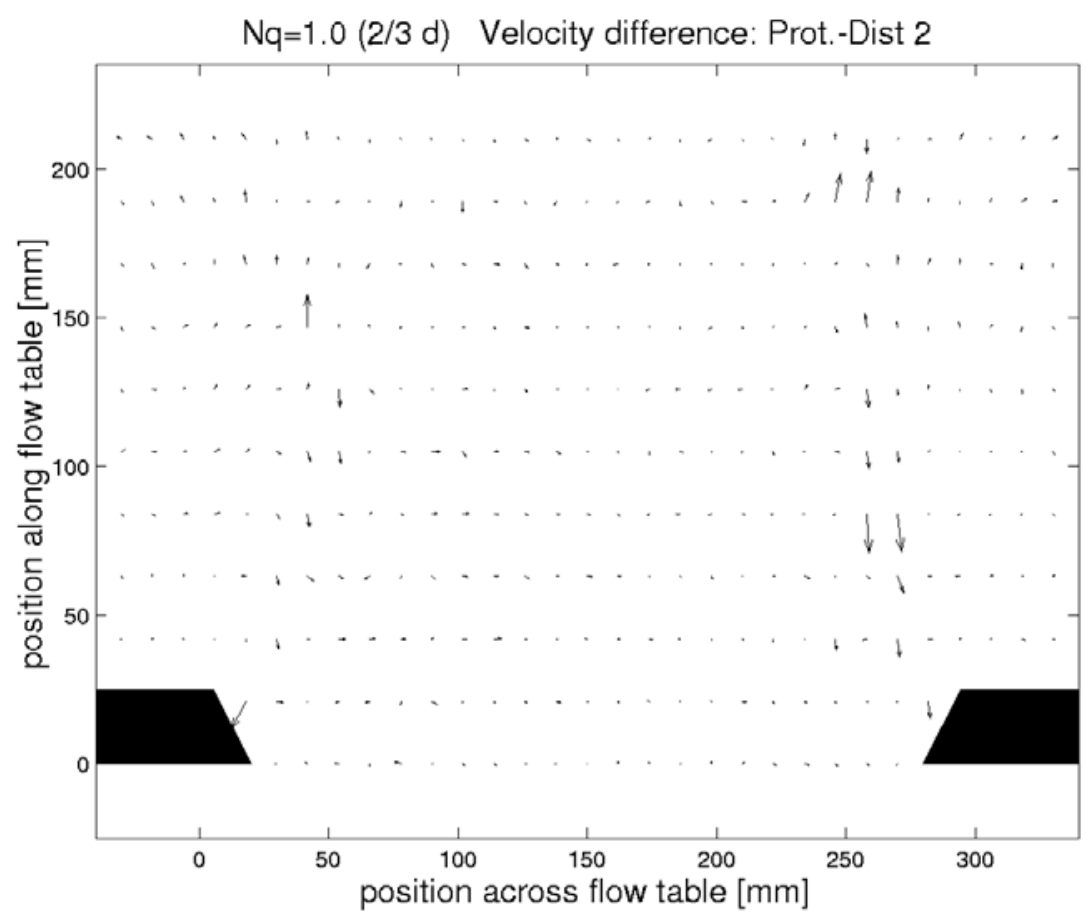

Figure C20. Case $3(2 / 3 d), Q=1.5 \mathrm{~L} / \mathrm{sec}$, prototype minus distortion = 2 


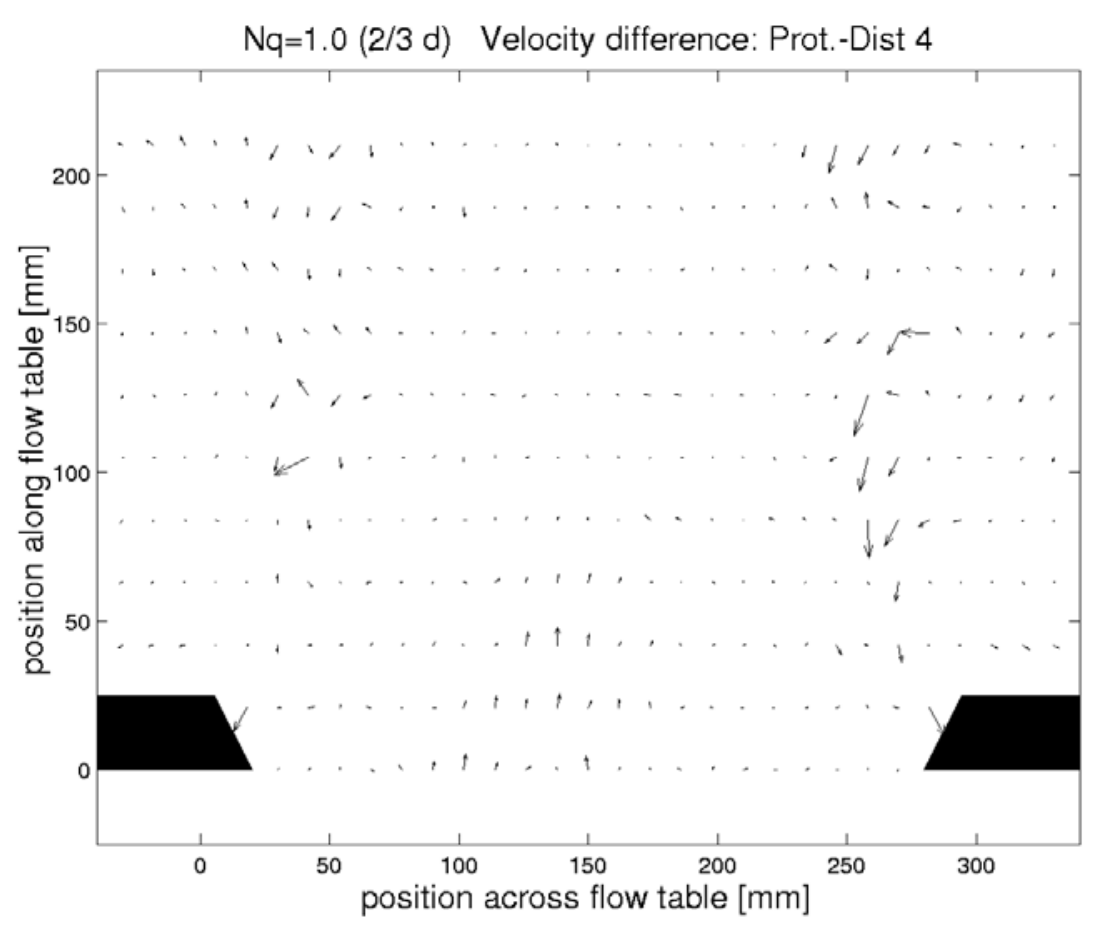

Figure C21. Case $3(2 / 3 d), Q=1.5 \mathrm{~L} / \mathrm{sec}$, prototype minus distortion $=4$

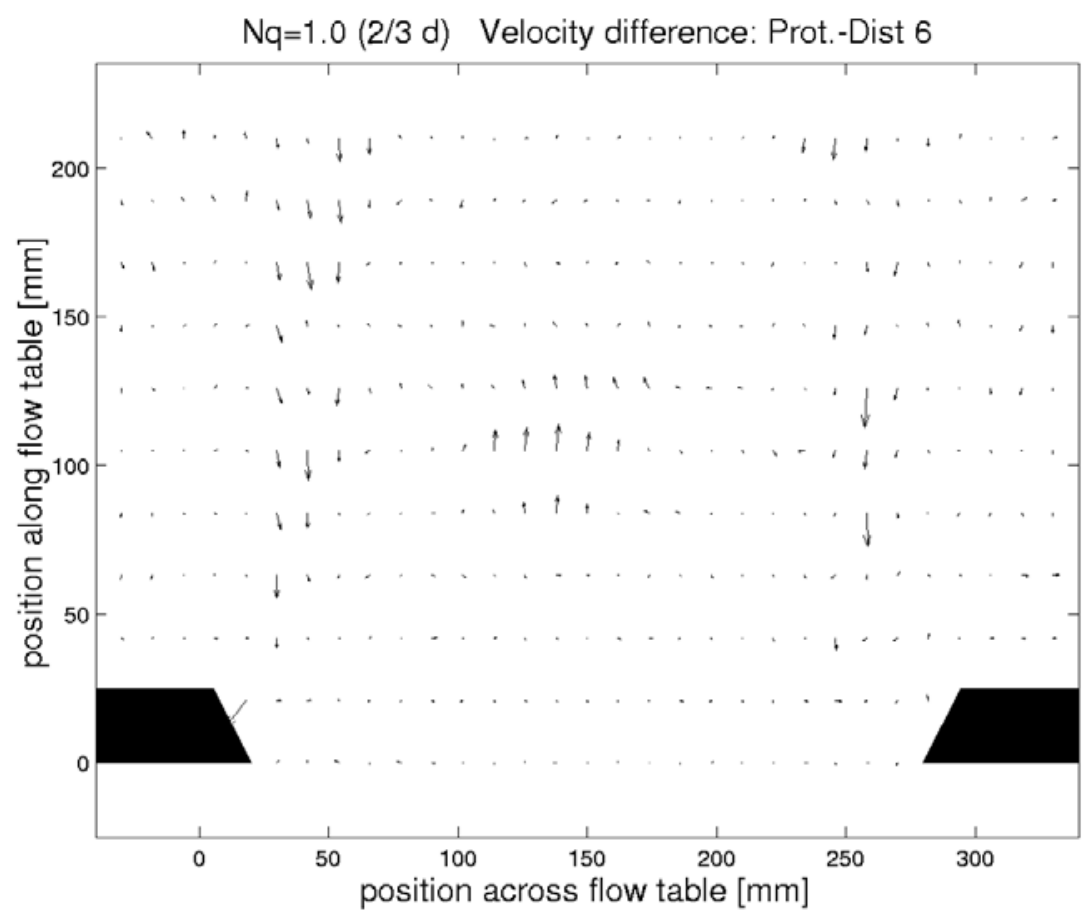

Figure C22. Case $3(2 / 3 d), Q=1.5 \mathrm{~L} / \mathrm{sec}$, prototype minus distortion $=6$ 


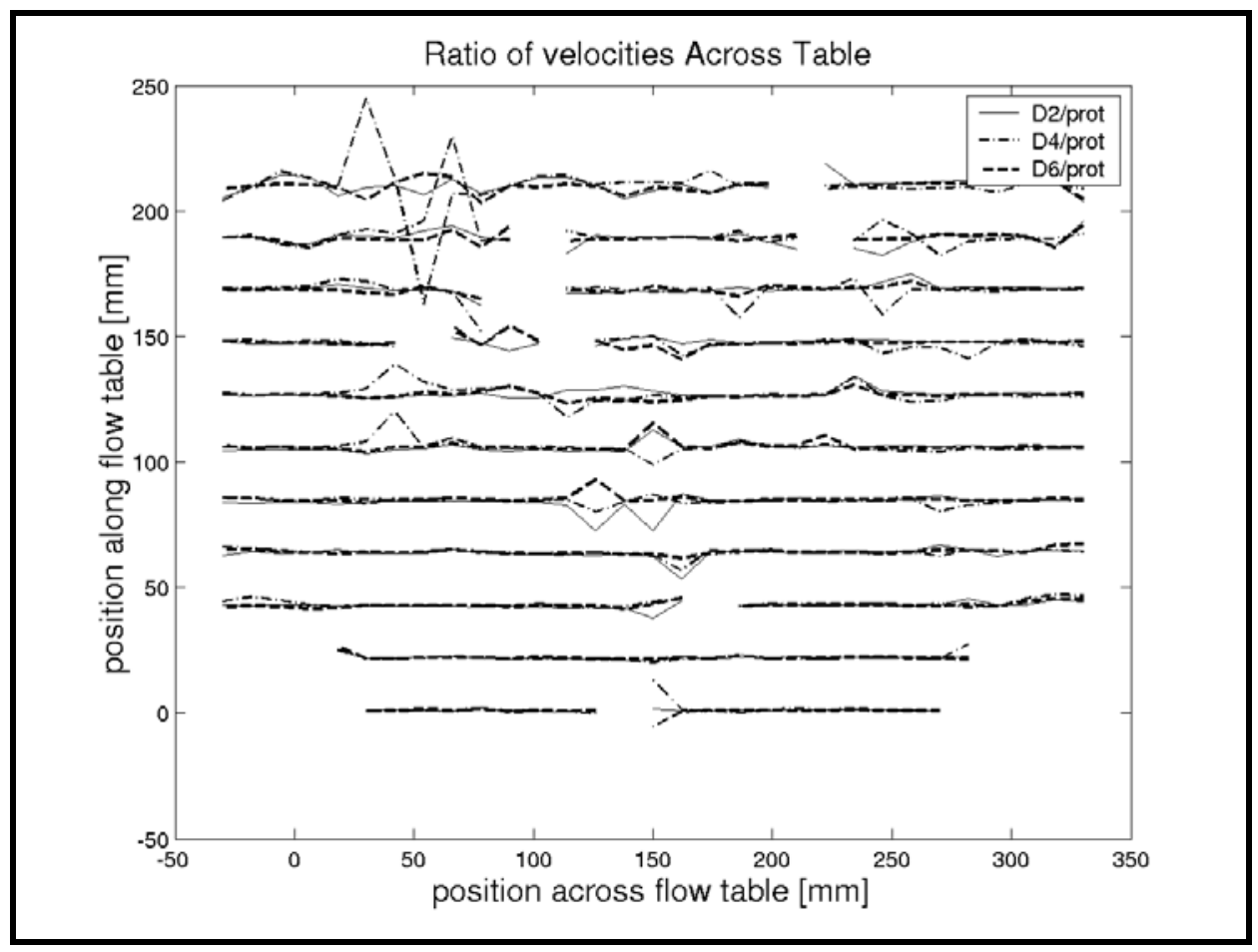

Figure C23. Case $3(2 / 3 d), Q=1.5 \mathrm{~L} / \mathrm{sec}$, ratios between prototype and distortions 2,4 , and 6

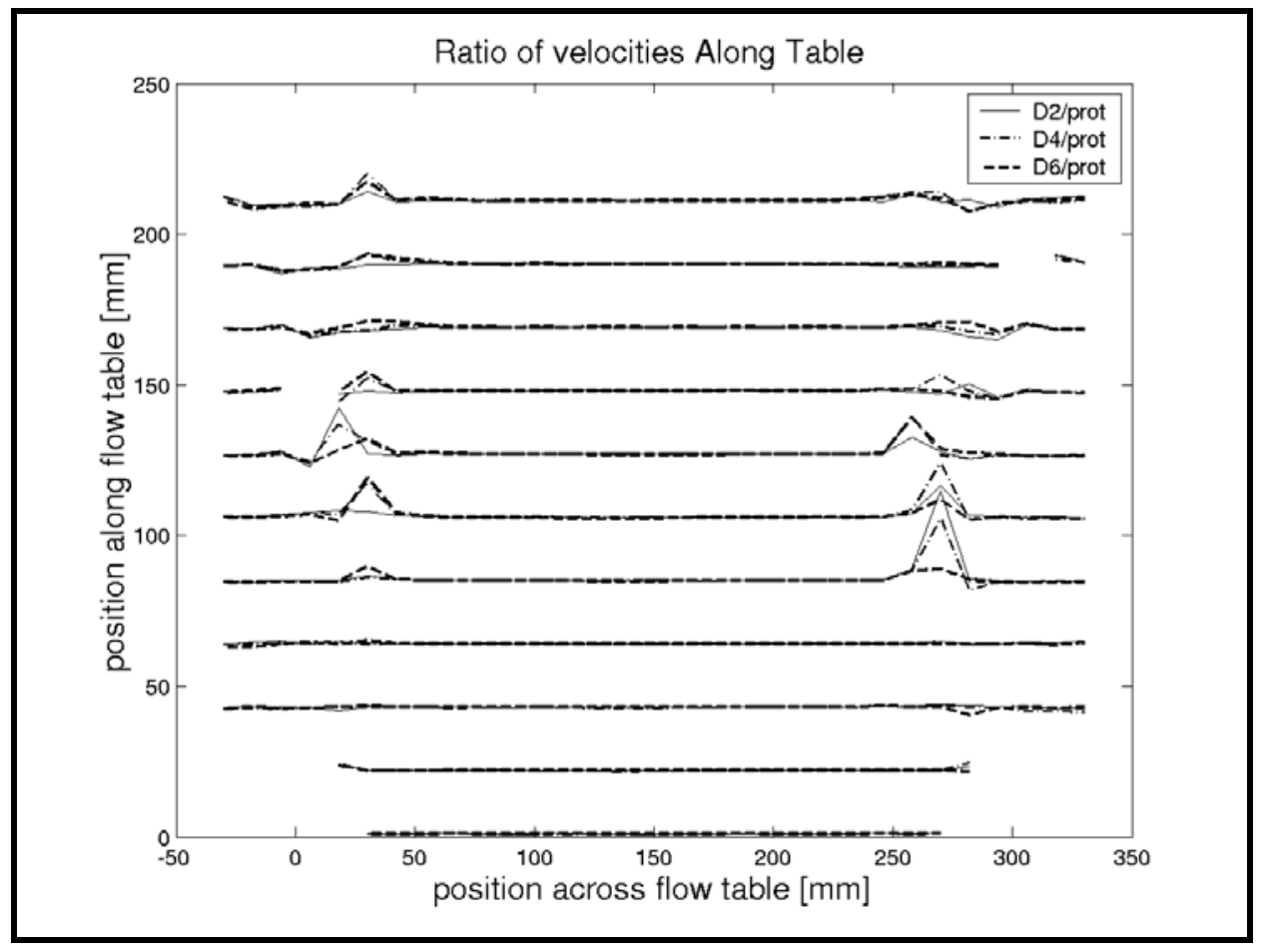

Figure C24. Case $3(2 / 3 d), Q=1.5 \mathrm{~L} / \mathrm{sec}$, ratios between prototype and distortions 2,4 , and 6 


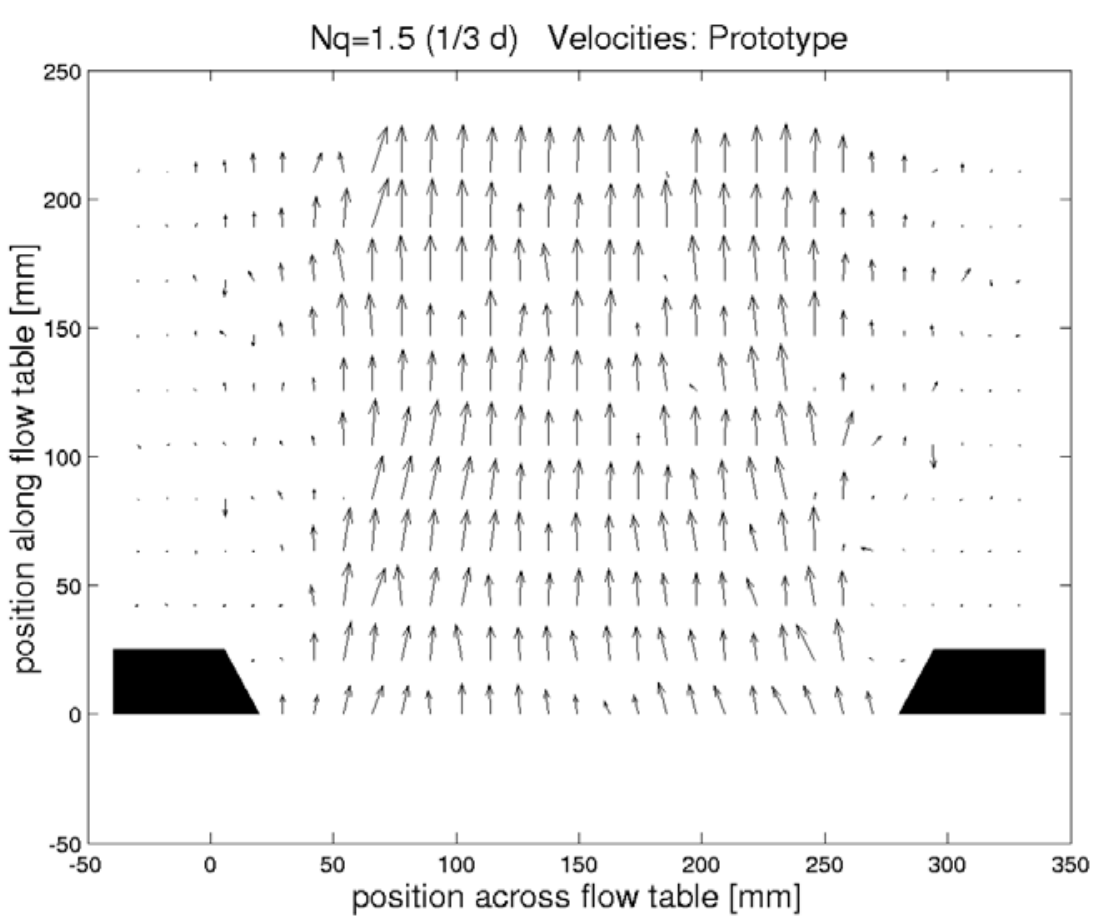

Figure C25. Case $3(1 / 3 d), Q=1.0 \mathrm{~L} / \mathrm{sec}$, prototype

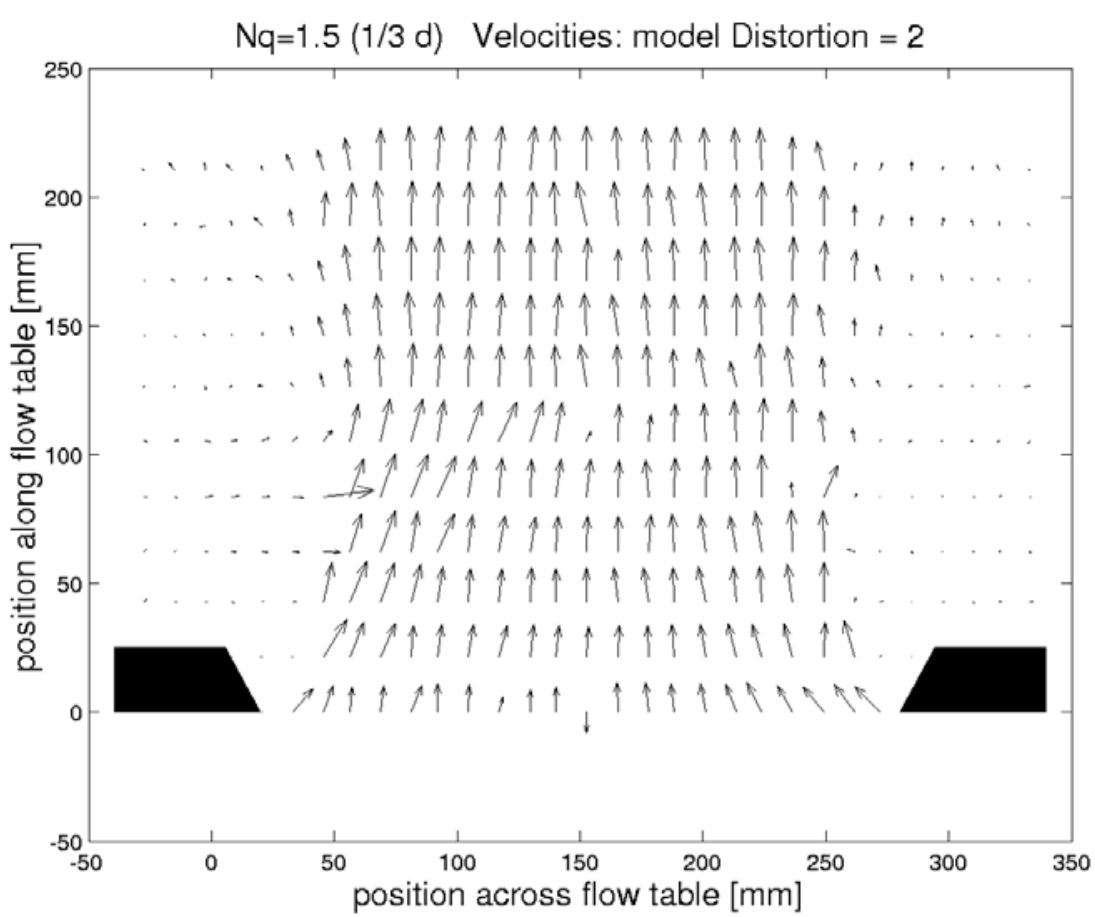

Figure C26. Case $3(1 / 3 d), Q=1.0 \mathrm{~L} / \mathrm{sec}$, distortion $=2$ 


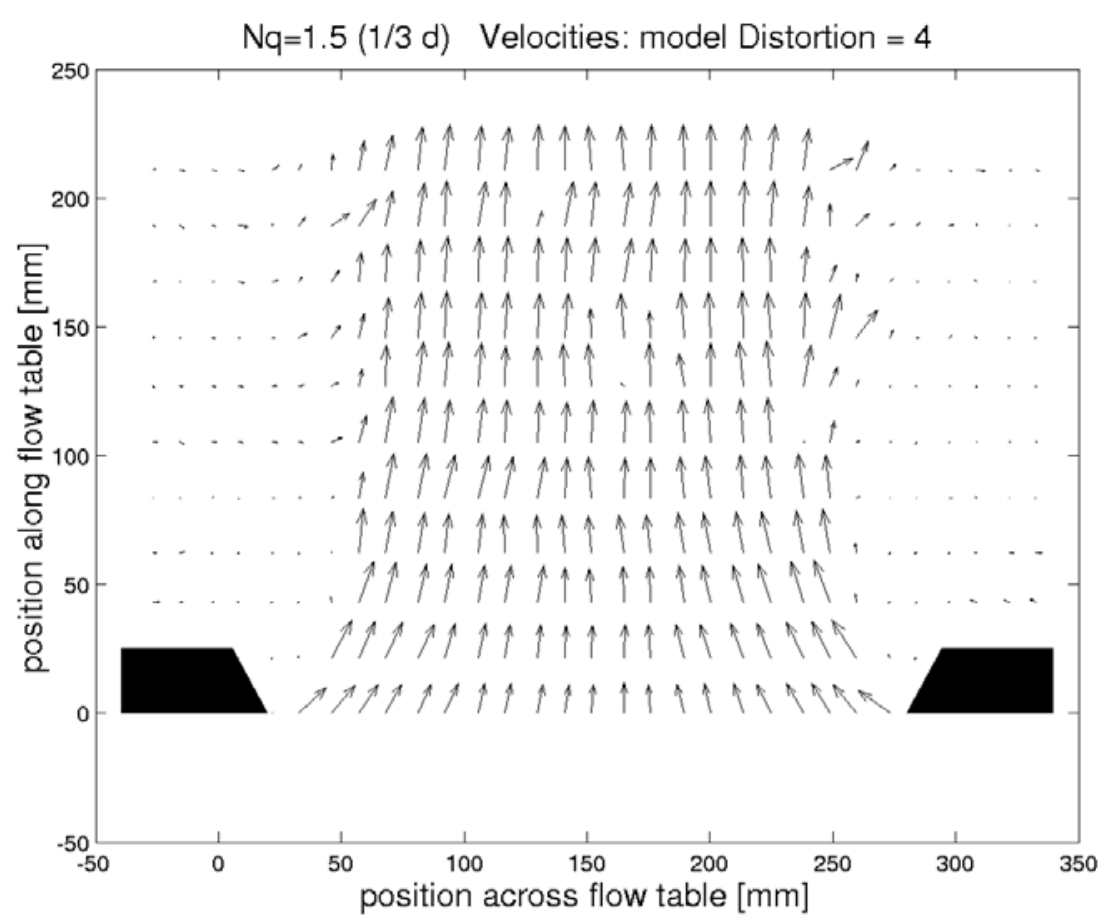

Figure C27. Case $3(1 / 3 d), Q=1.0 \mathrm{~L} / \mathrm{sec}$, distortion $=4$

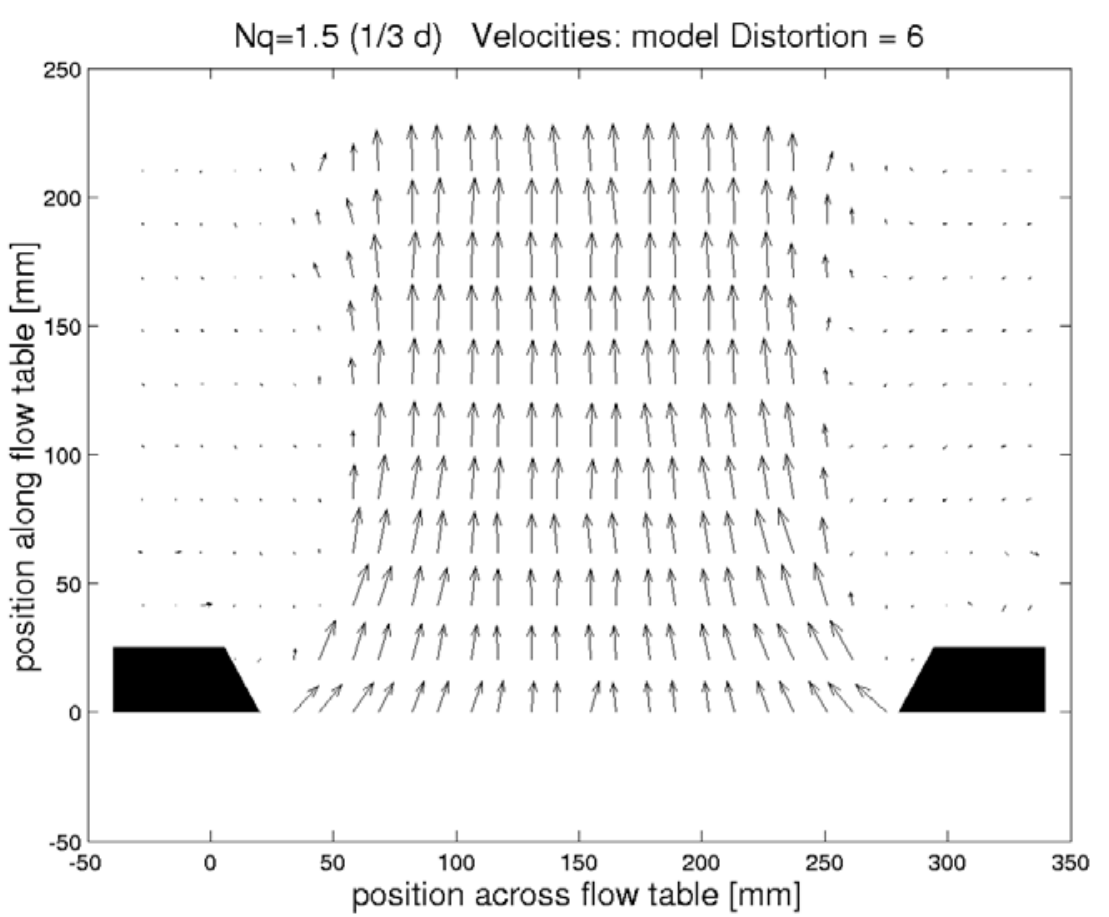

Figure C28. Case $3(1 / 3 d), Q=1.0 \mathrm{~L} / \mathrm{sec}$, distortion $=6$ 


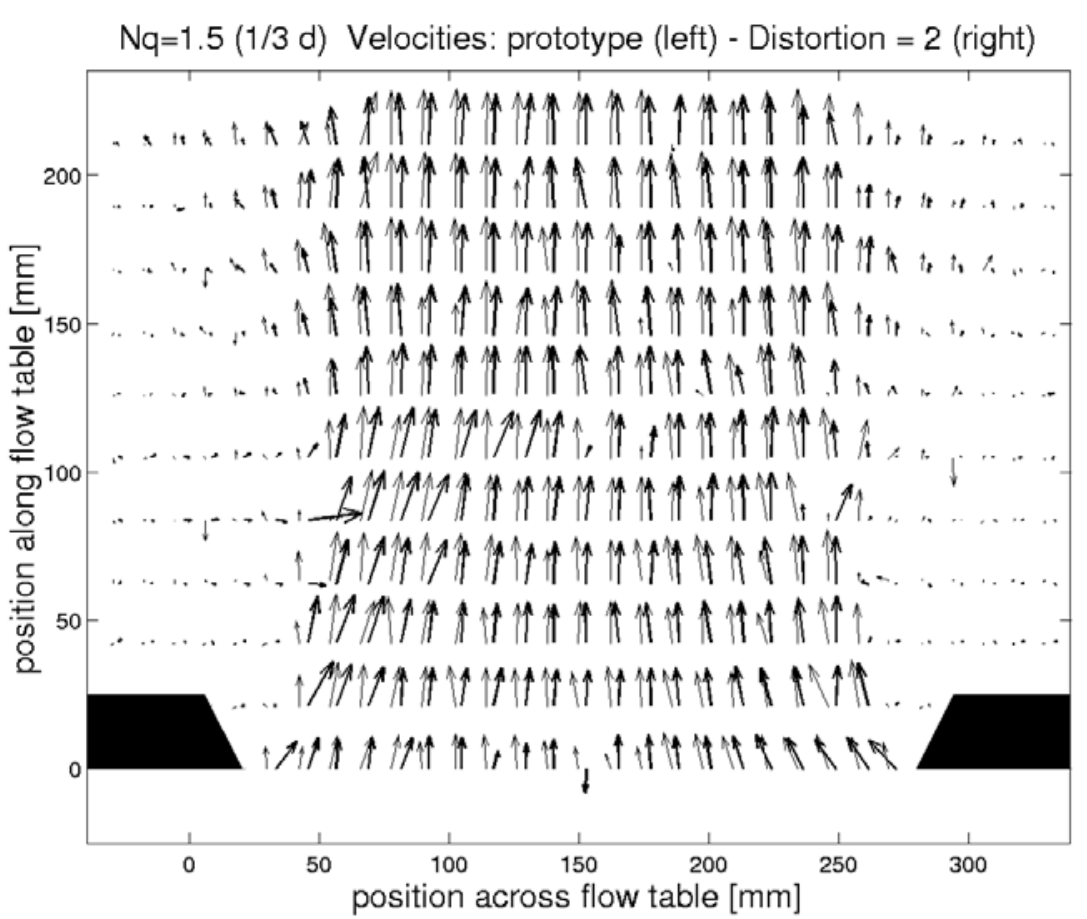

Figure C29. Case $3(1 / 3 d), Q=1.0 \mathrm{~L} / \mathrm{sec}$, prototype vs. distortion $=2$

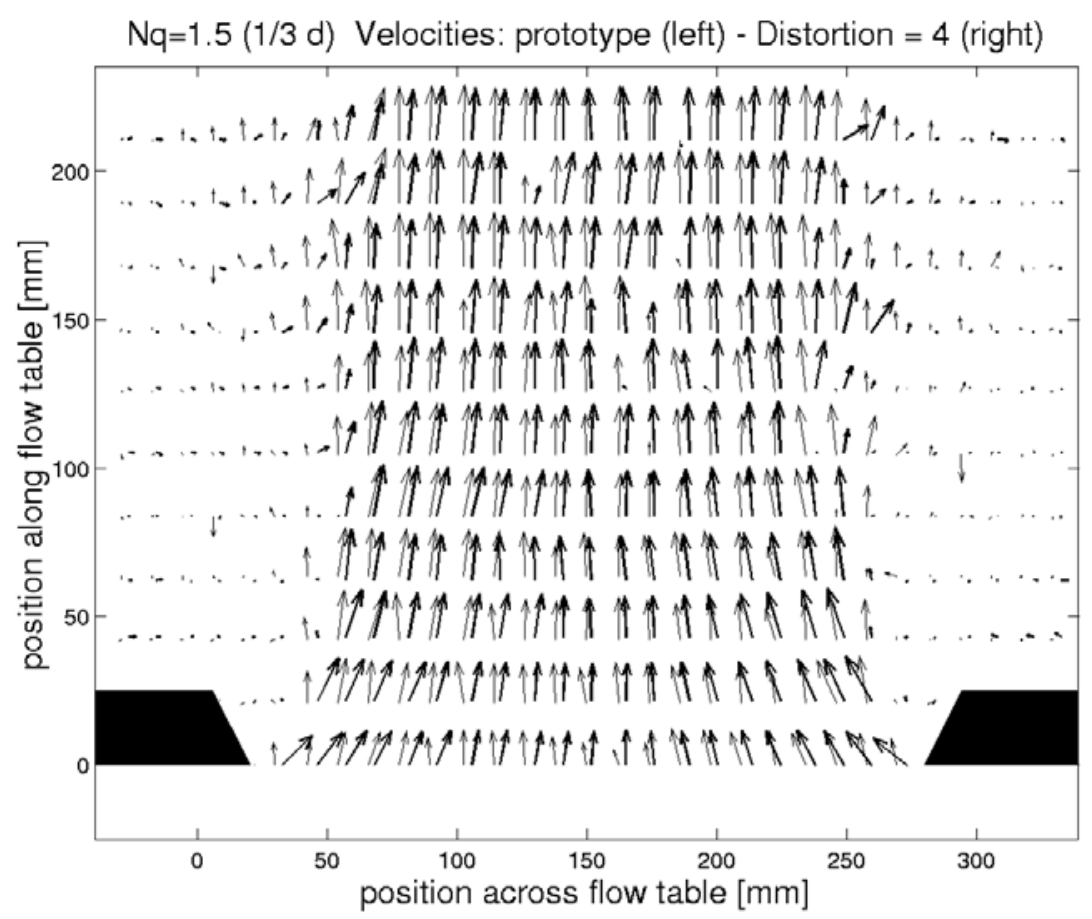

Figure C30. Case $3(1 / 3 d), Q=1.0 \mathrm{~L} / \mathrm{sec}$, prototype vs. distortion $=4$ 


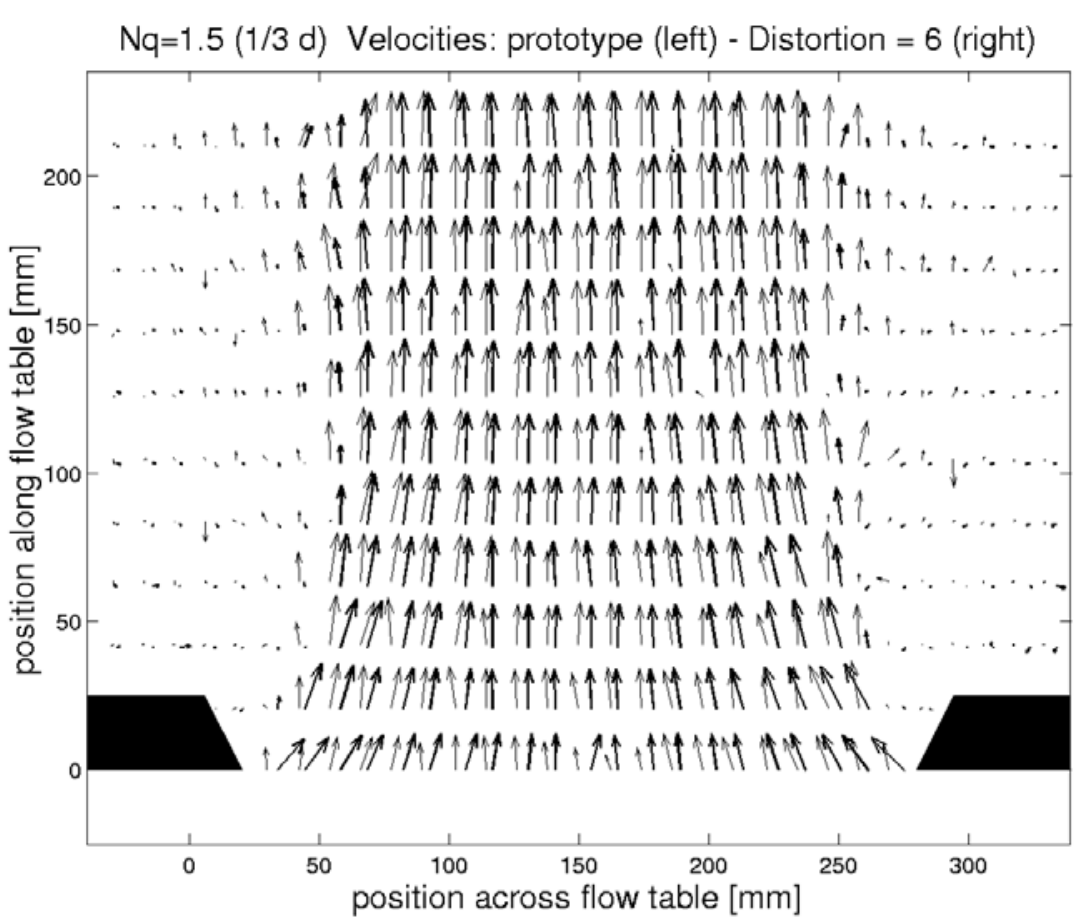

Figure C31. Case $3(1 / 3 d), Q=1.0 \mathrm{~L} / \mathrm{sec}$, prototype vs. distortion $=6$

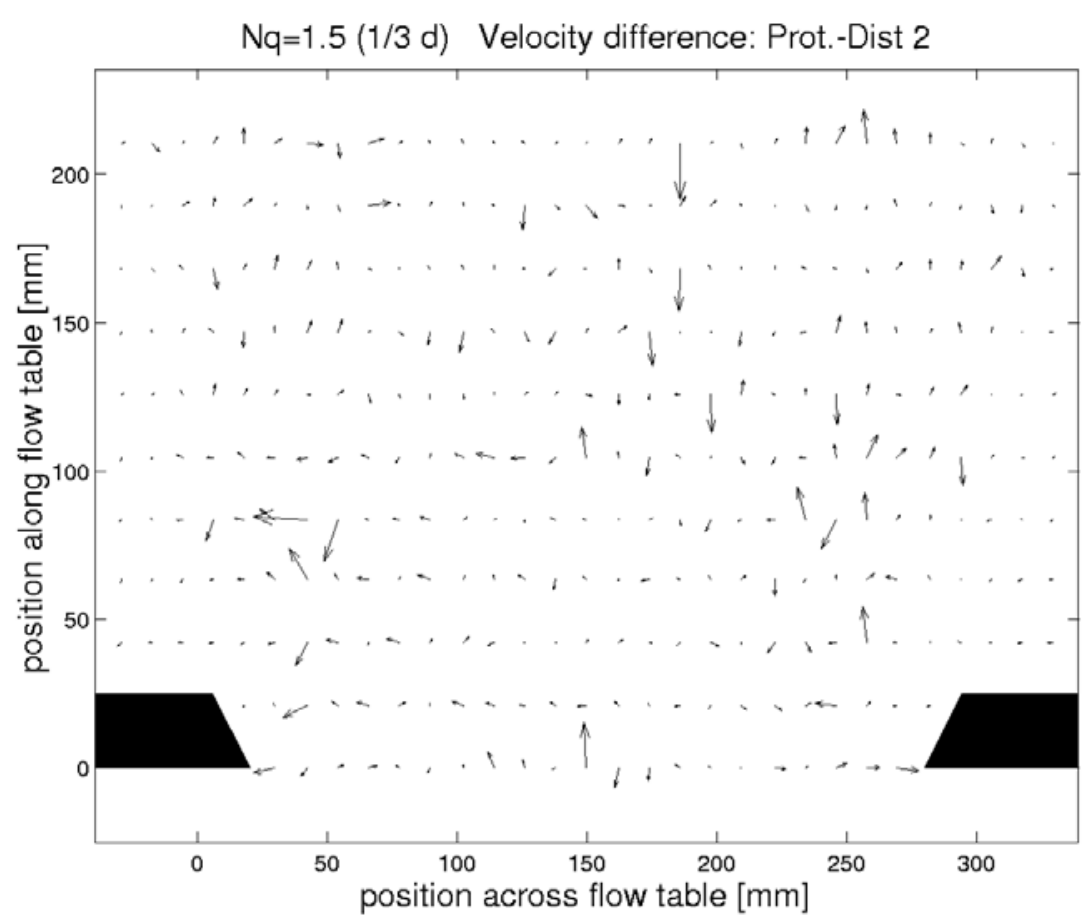

Figure C32. Case $3(1 / 3 d), Q=1.0 \mathrm{~L} / \mathrm{sec}$, prototype minus distortion $=2$ 


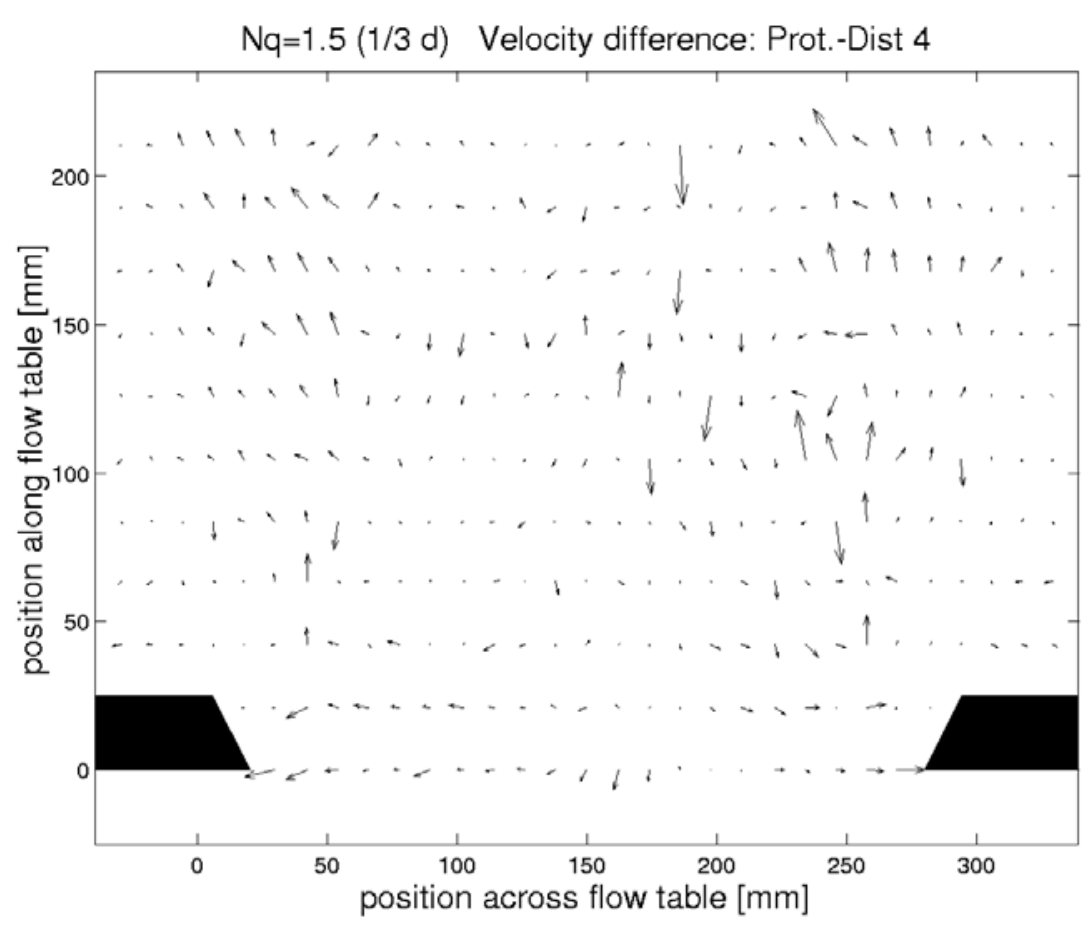

Figure C33. Case $3(1 / 3 d), Q=1.0 \mathrm{~L} / \mathrm{sec}$, prototype minus distortion $=4$

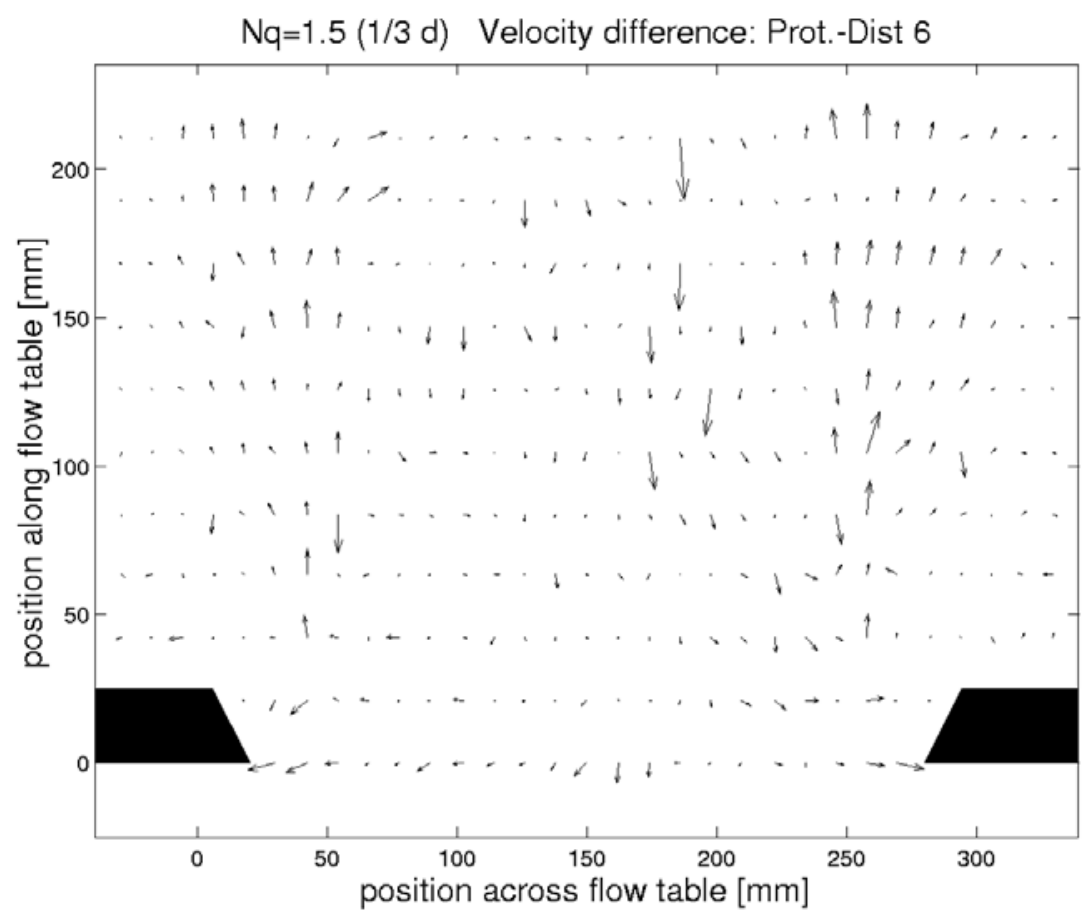

Figure C34. Case $3(1 / 3 d), Q=1.0 \mathrm{~L} / \mathrm{sec}$, prototype minus distortion = 6 


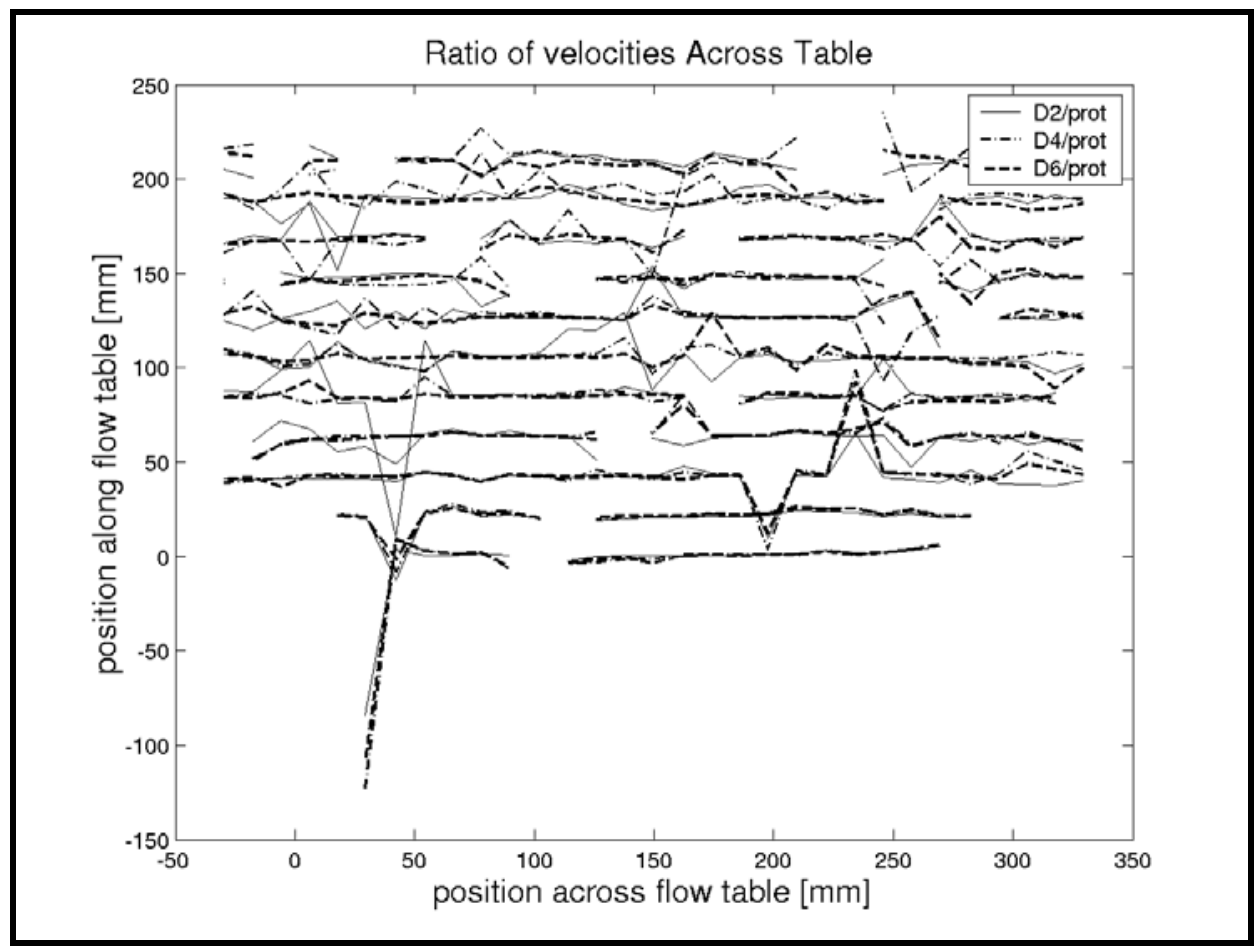

Figure C35. Case $3(1 / 3 d), Q=1.0 \mathrm{~L} / \mathrm{sec}$, ratios between prototype and distortions 2,4 , and 6

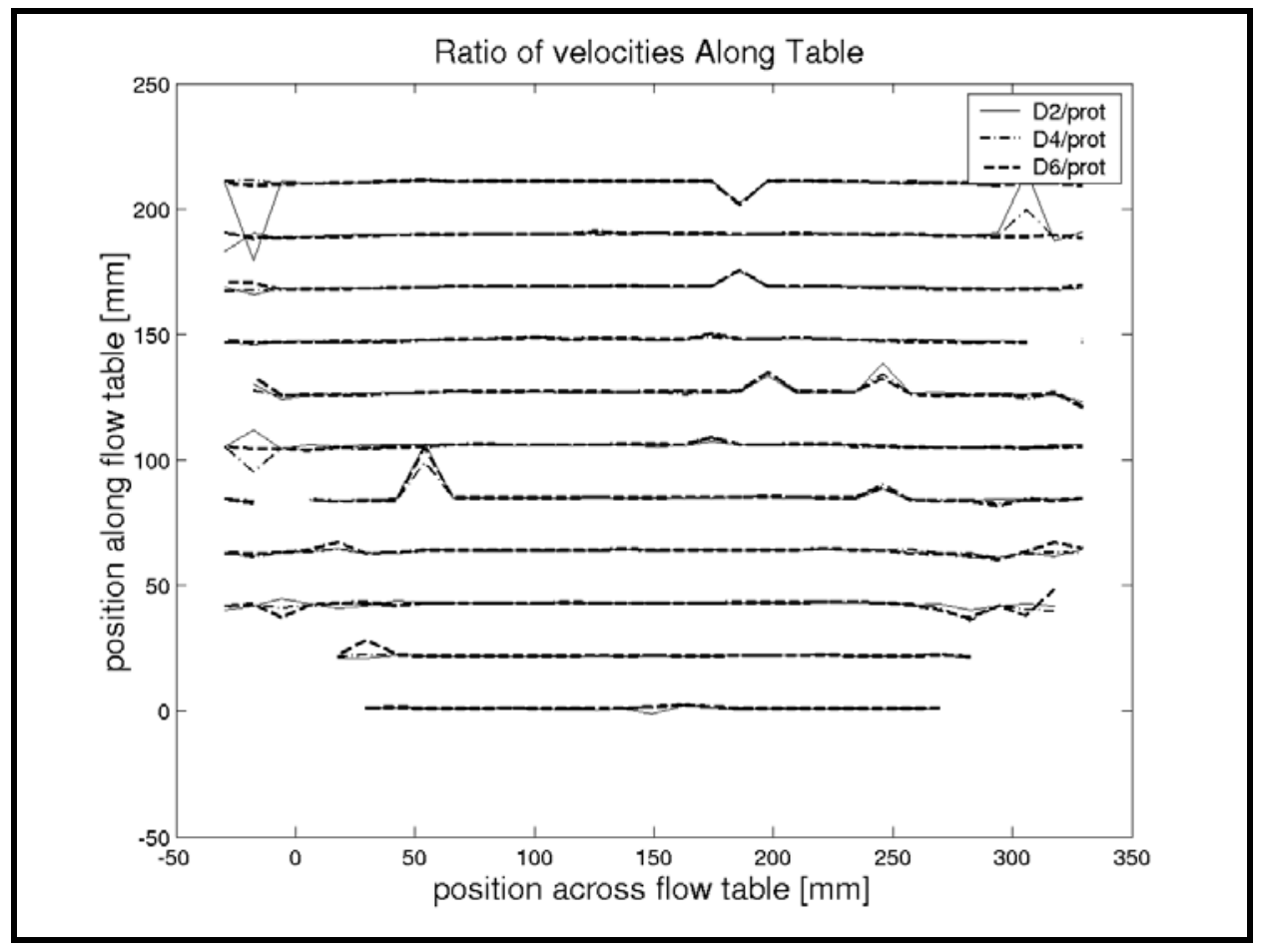

Figure C36. Case $3(1 / 3 d), Q=1.0 \mathrm{~L} / \mathrm{sec}$, ratios between prototype and distortions 2,4 , and 6 


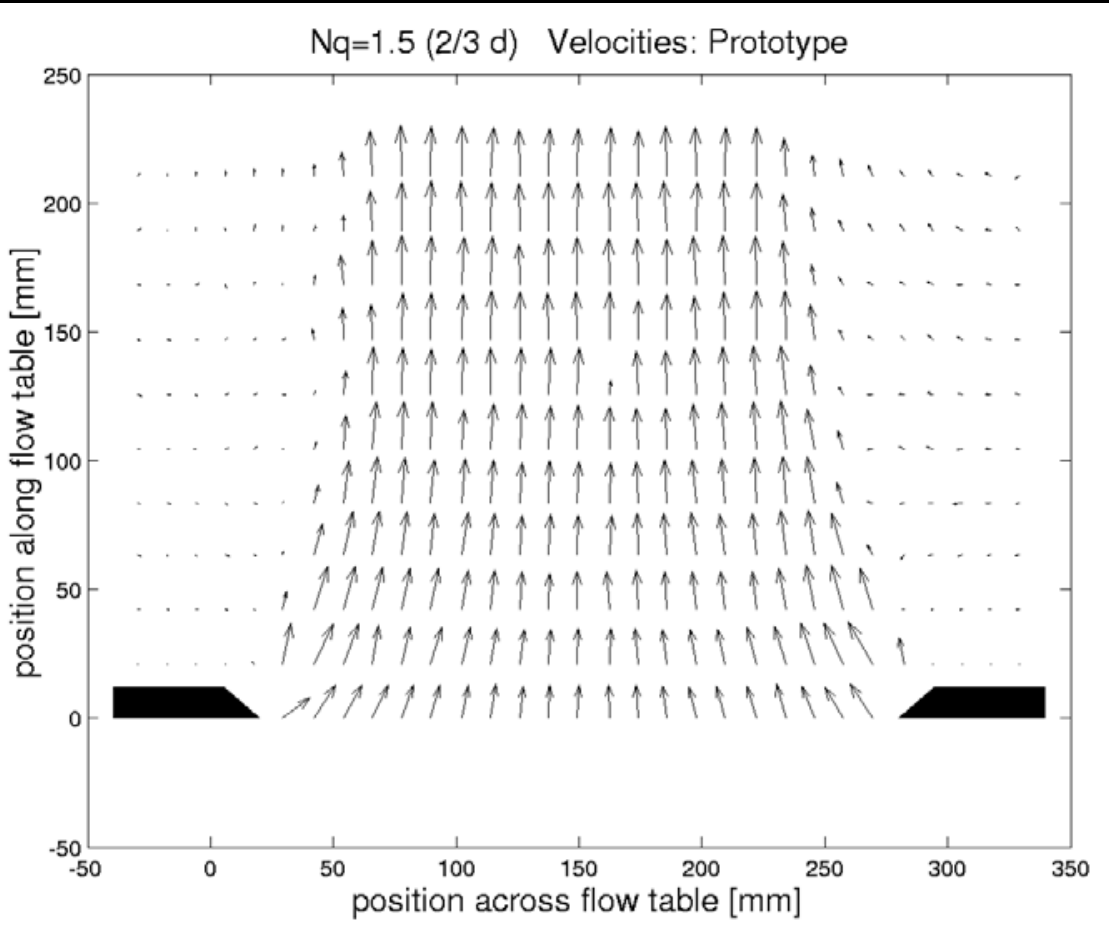

Figure C37. Case $3(2 / 3 d), Q=1.0 \mathrm{~L} / \mathrm{sec}$, prototype

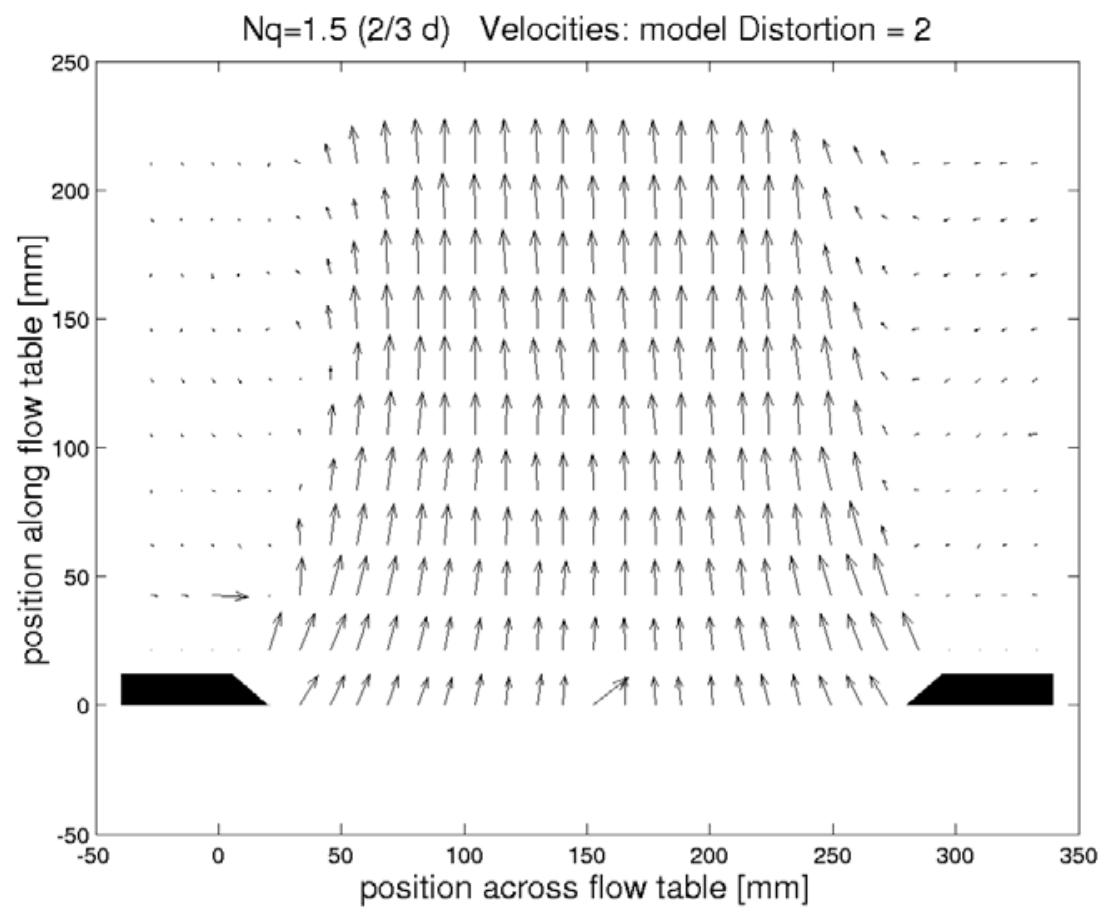

Figure C38. Case $3(2 / 3 d), Q=1.0 \mathrm{~L} / \mathrm{sec}$, distortion $=2$ 


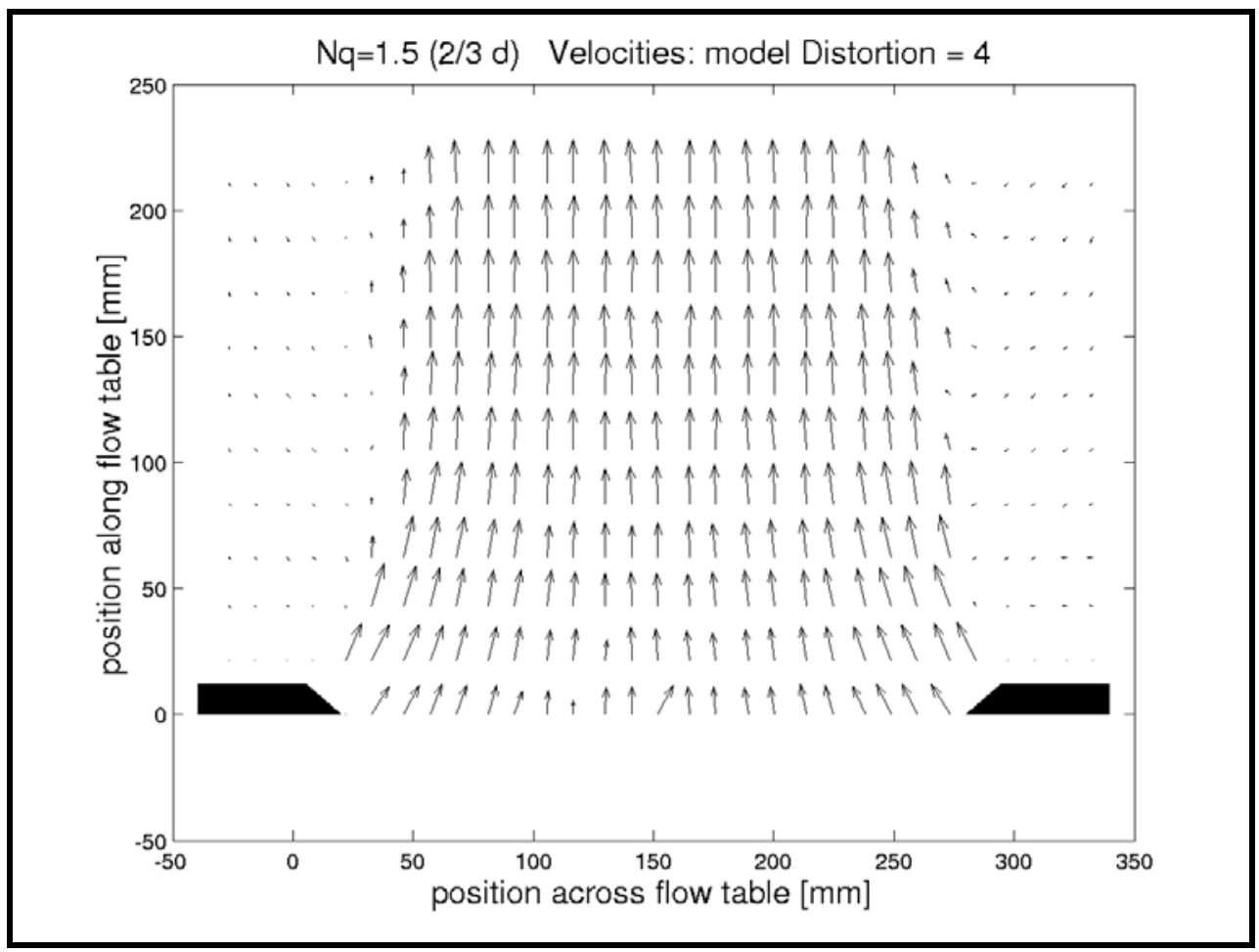

Figure C39. Case $3(2 / 3 d), Q=1.0 \mathrm{~L} / \mathrm{sec}$, distortion $=4$

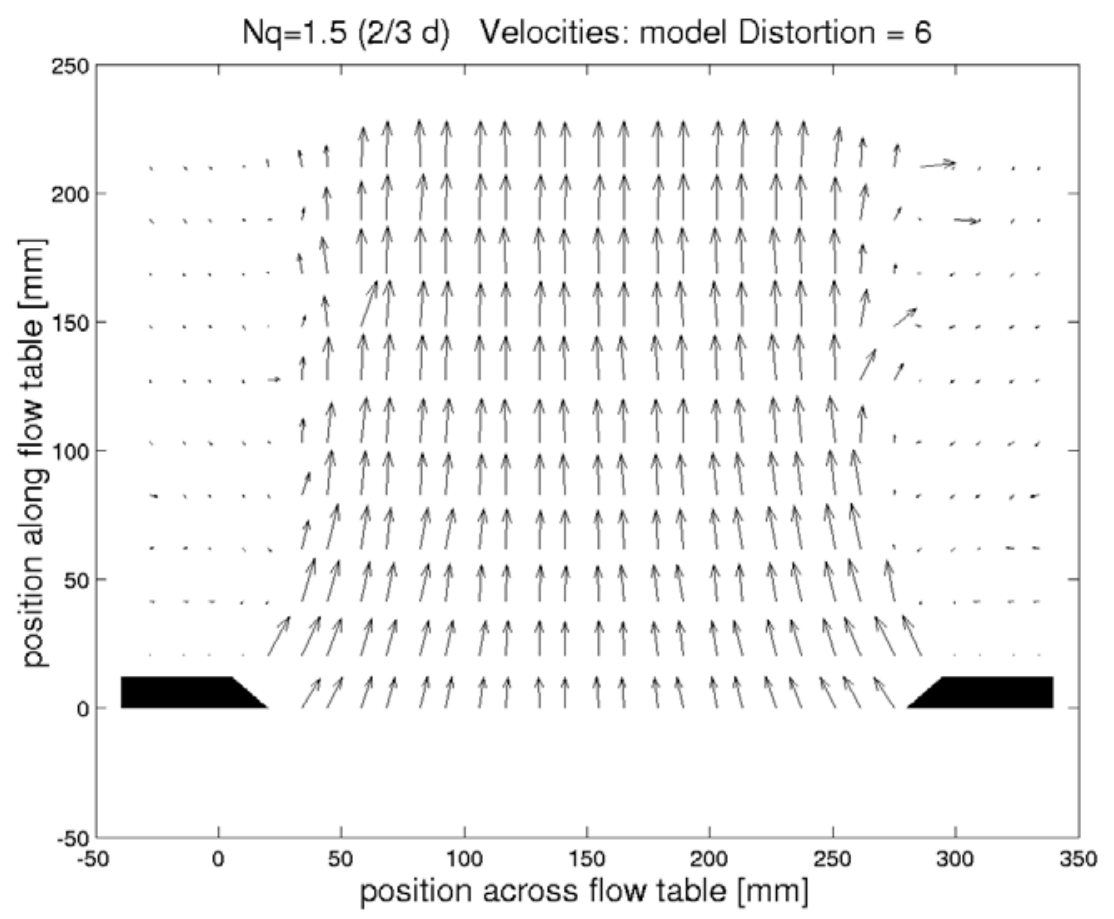

Figure C40. Case $3(2 / 3 d), Q=1.0 \mathrm{~L} / \mathrm{sec}$, distortion $=6$ 


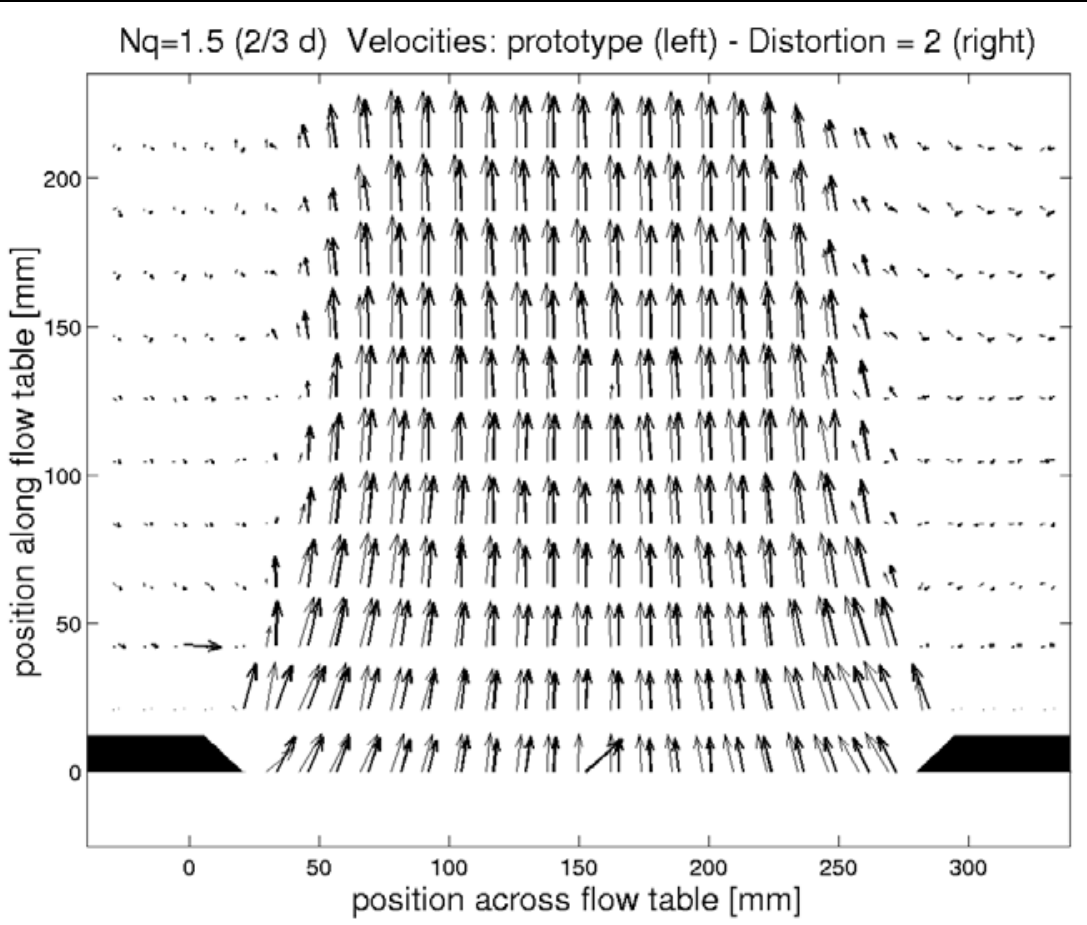

Figure C41. Case $3(2 / 3 d), Q=1.0 \mathrm{~L} / \mathrm{sec}$, prototype vs. distortion $=2$

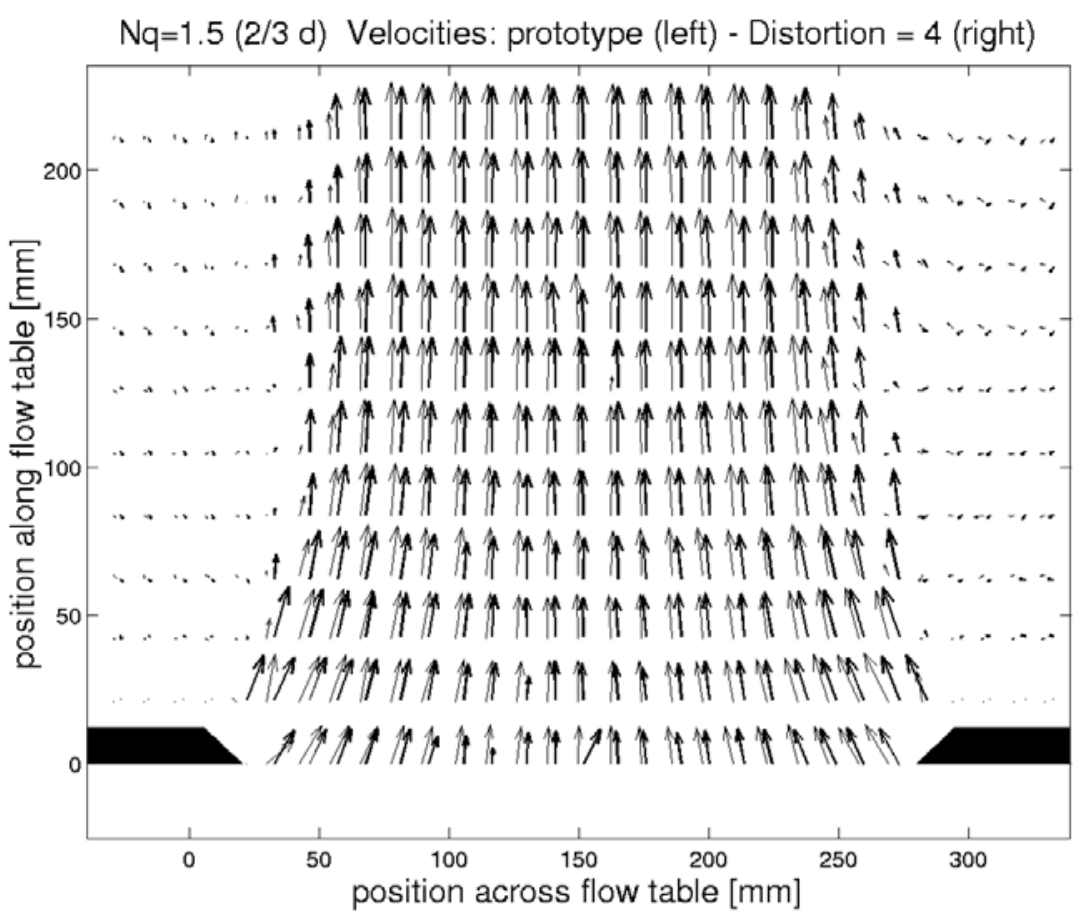

Figure C42. Case $3(2 / 3 d), Q=1.0 \mathrm{~L} / \mathrm{sec}$, prototype vs. distortion $=4$ 


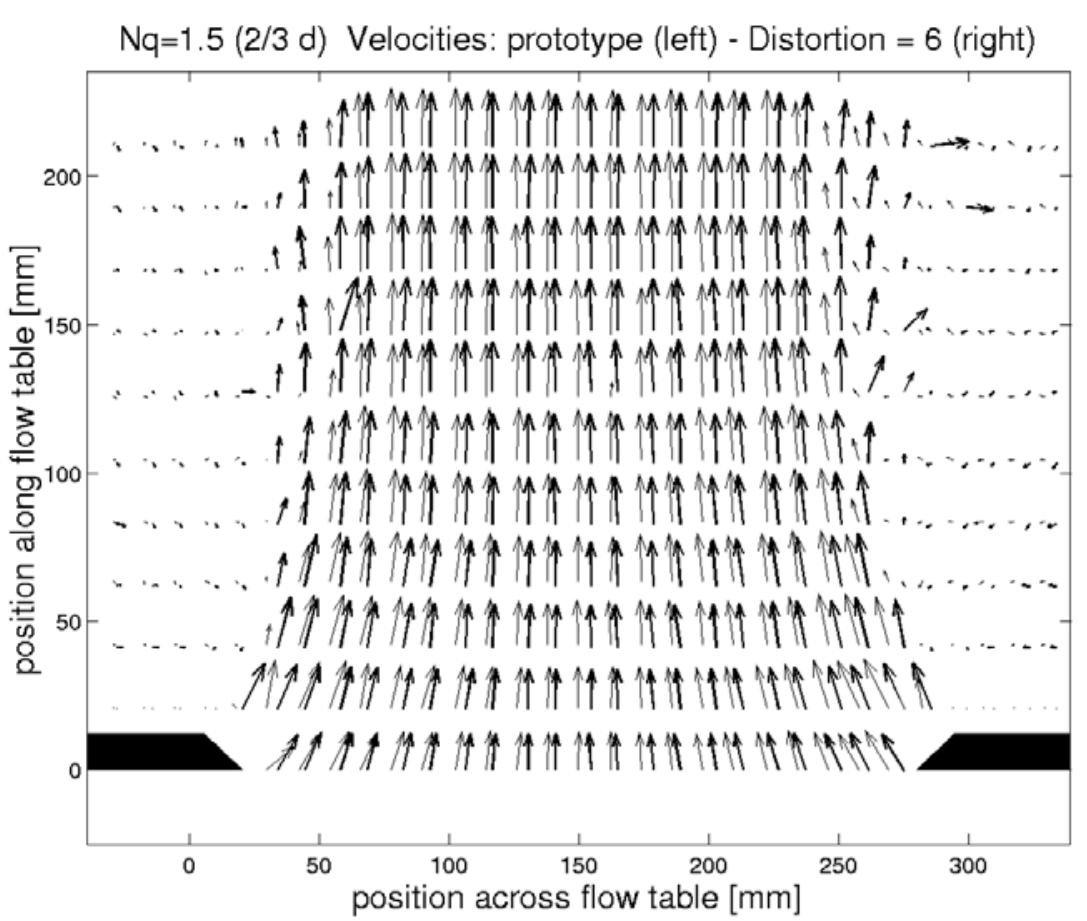

Figure C43. Case $3(2 / 3 d), Q=1.0 \mathrm{~L} / \mathrm{sec}$, prototype vs. distortion $=6$

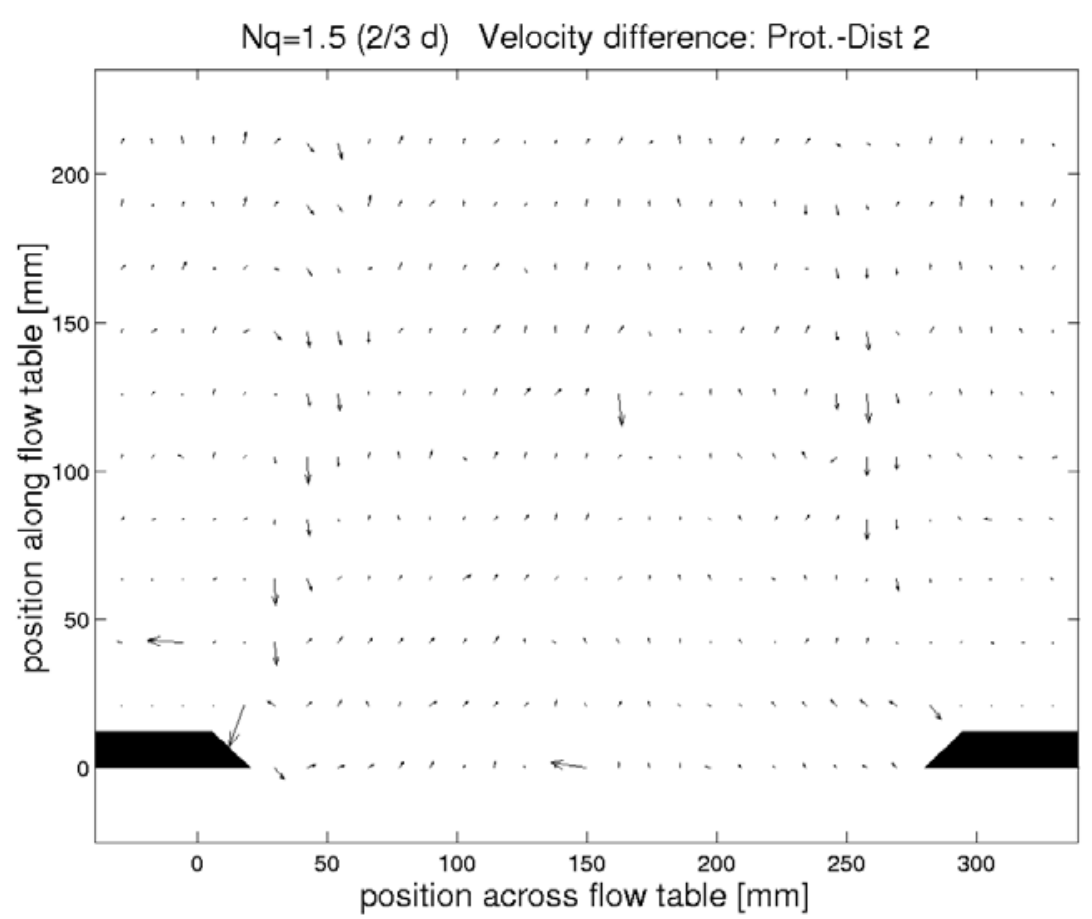

Figure C44. Case $3(2 / 3 d), Q=1.0 \mathrm{~L} / \mathrm{sec}$, prototype minus distortion $=2$ 


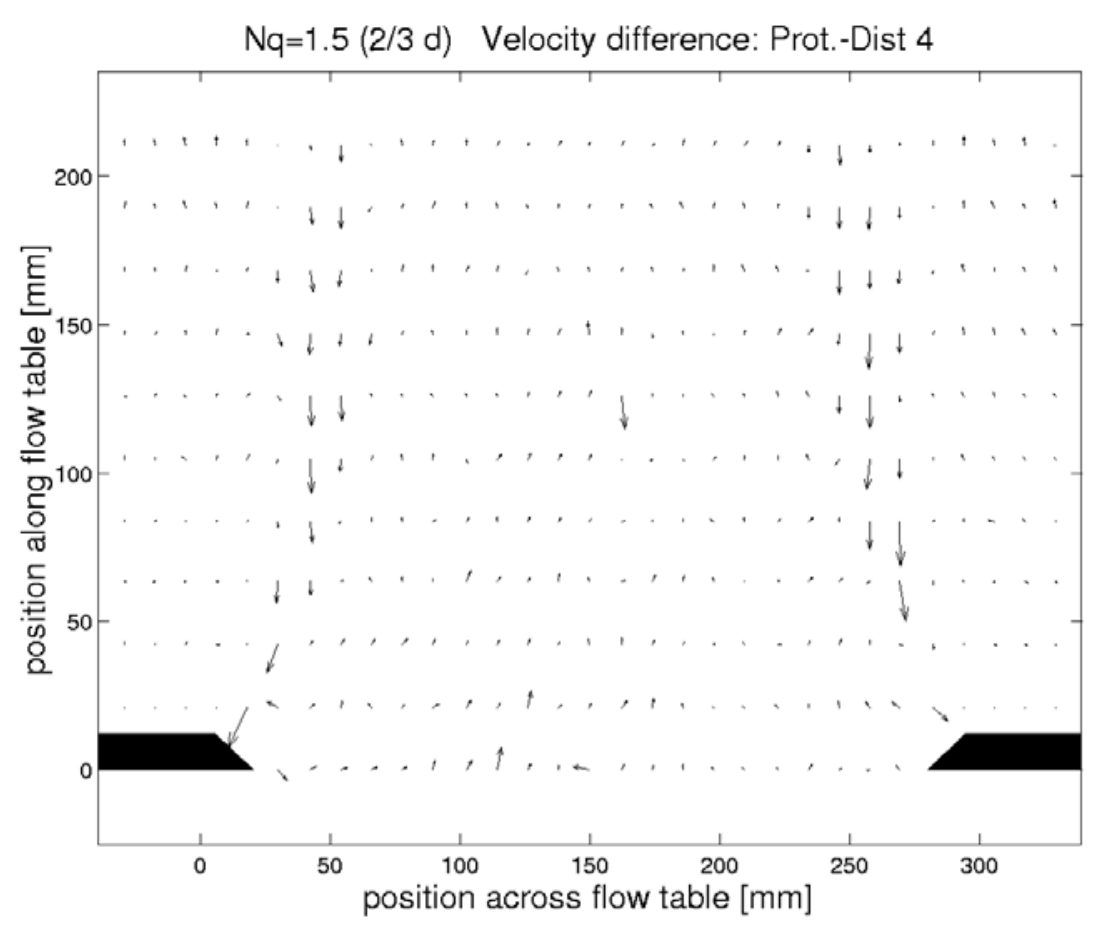

Figure C45. Case $3(2 / 3 d), Q=1.0 \mathrm{~L} / \mathrm{sec}$, prototype minus distortion $=4$

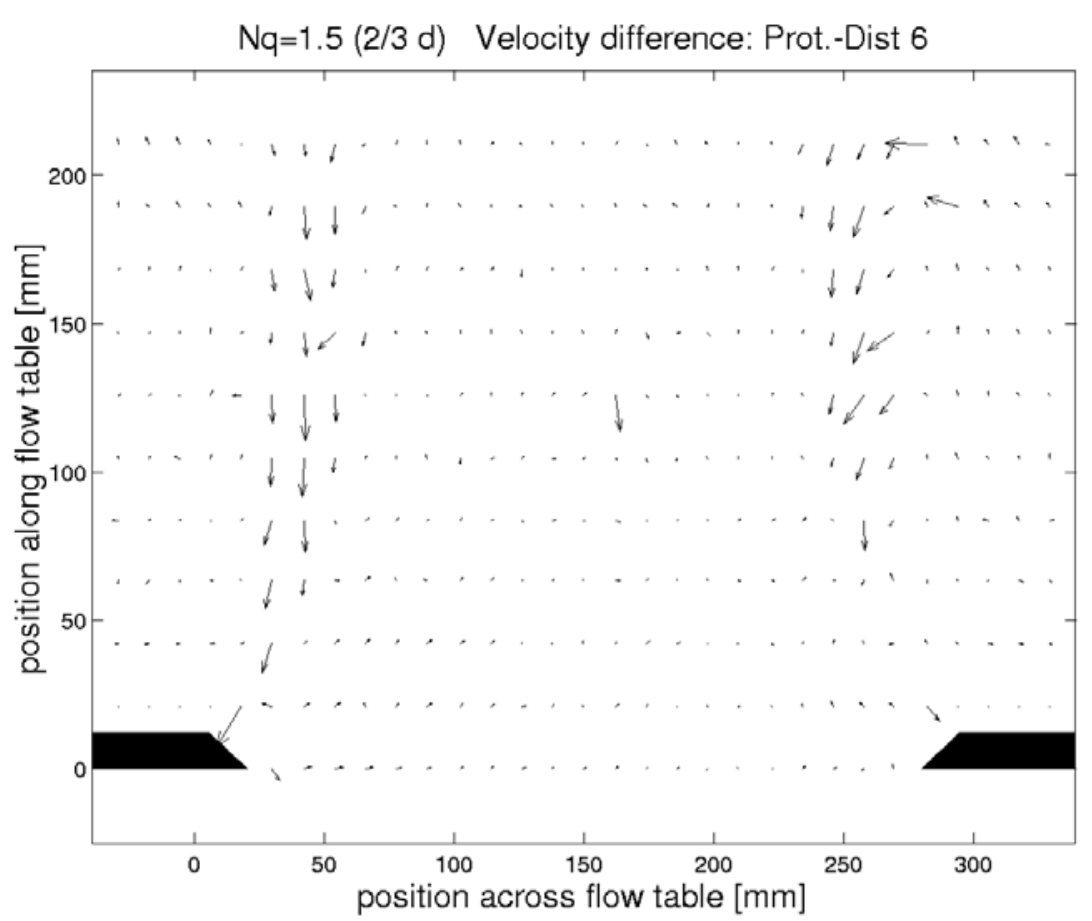

Figure C46. Case $3(2 / 3 d), Q=1.0 \mathrm{~L} / \mathrm{sec}$, prototype minus distortion $=6$ 


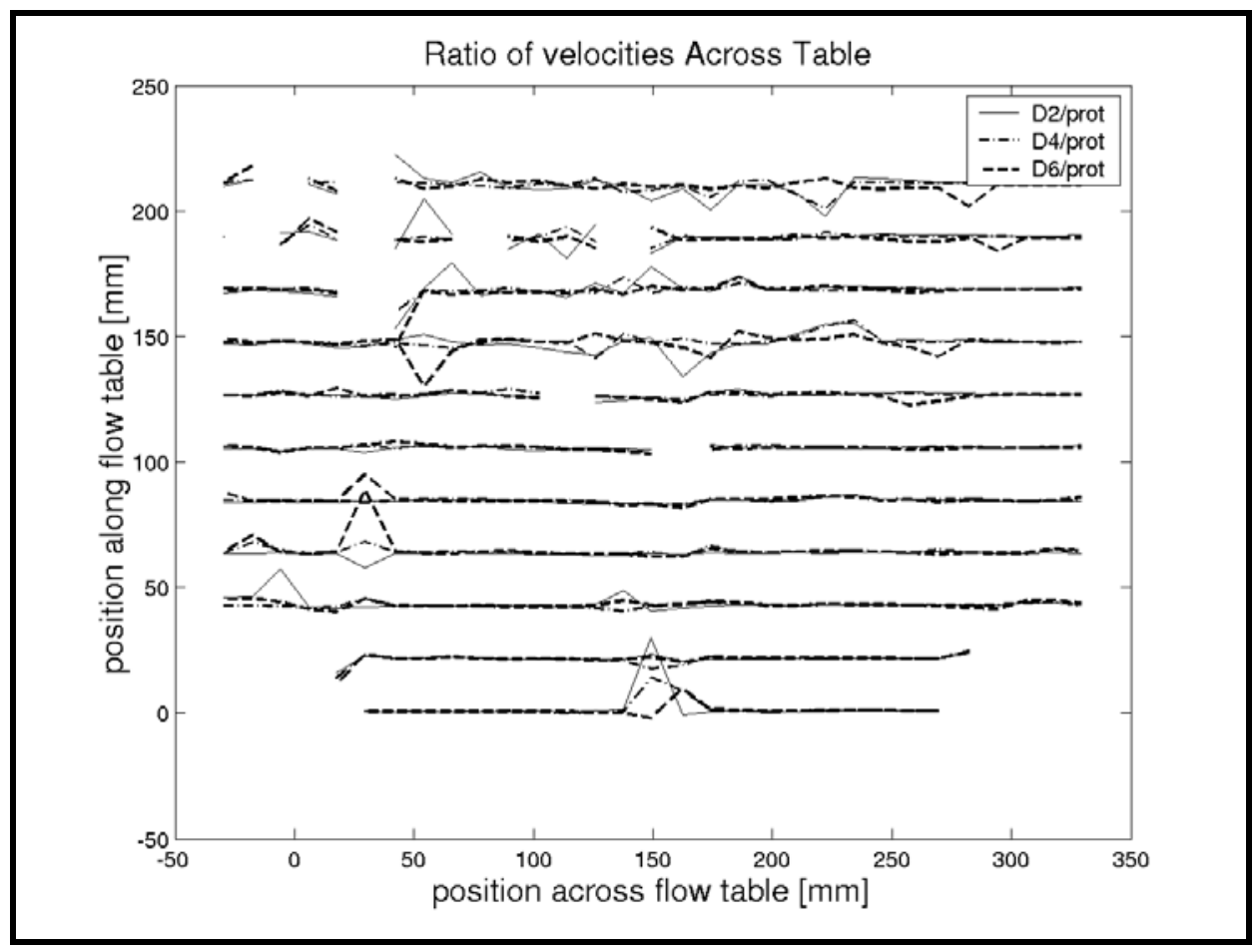

Figure C47. Case $3(2 / 3 d), Q=1.0 \mathrm{~L} / \mathrm{sec}$, ratios between prototype and distortions 2,4 , and 6

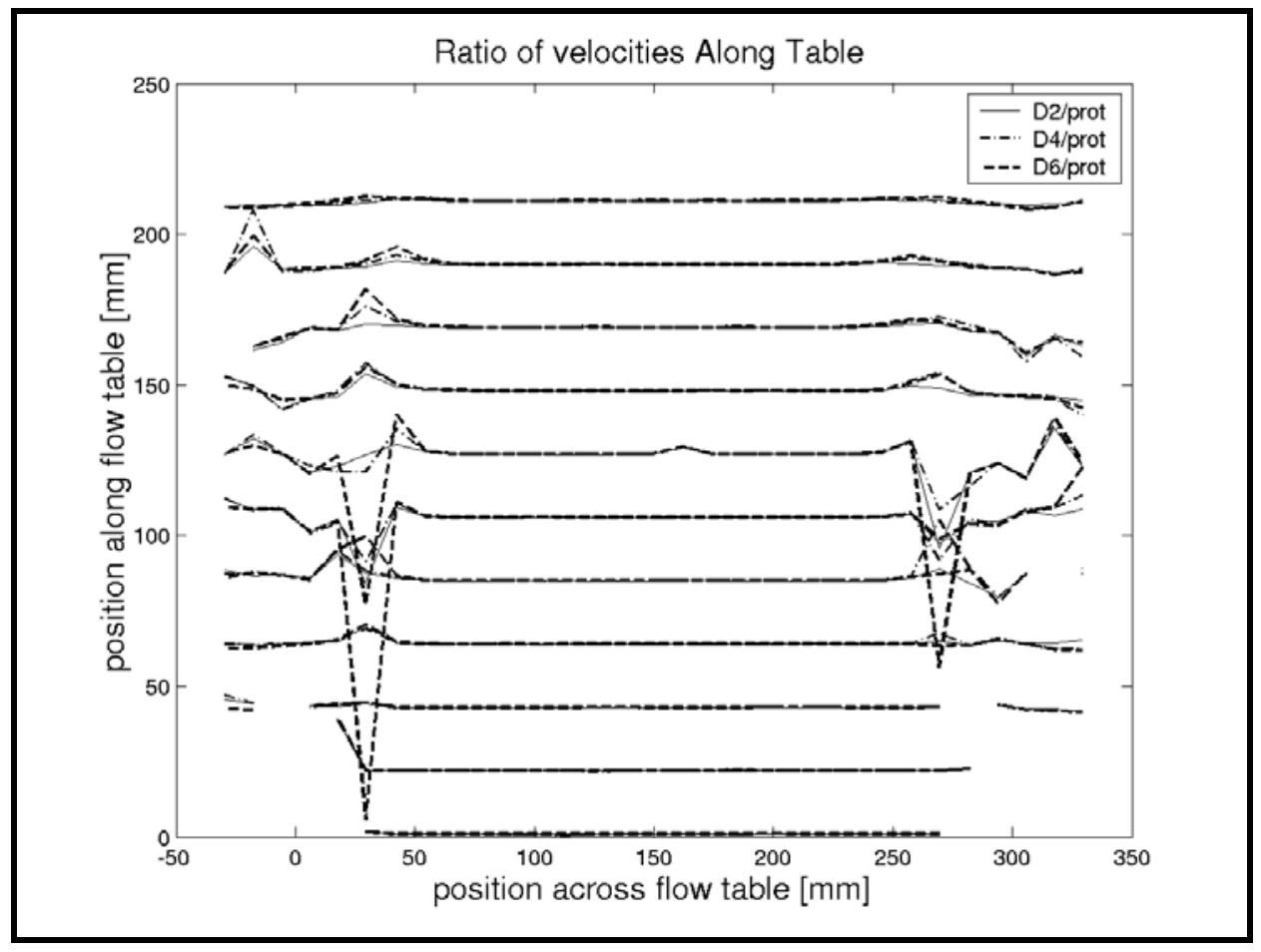

Figure C48. Case $3(2 / 3 d), Q=1.0 \mathrm{~L} / \mathrm{sec}$, ratios between prototype and distortions 2,4 , and 6 


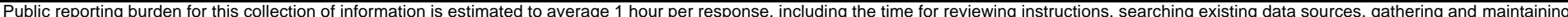

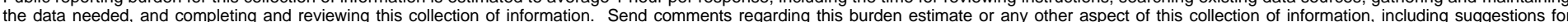

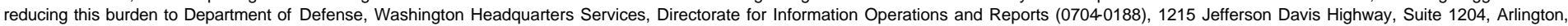

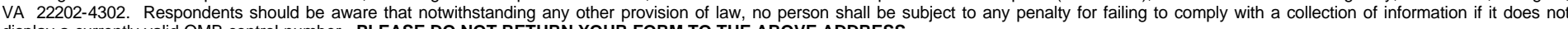
display a currently valid OMB control number. PLEASE DO NOT RETURN YOUR FORM TO THE ABOVE ADDRESS.

\begin{tabular}{l|l|l}
\hline 1. REPORT DATE (DD-MM- $Y Y Y Y)$ & 2. REPORT TYPE & 3. DATES COVERED (FrOm - TO)
\end{tabular} July 2003

\section{TITLE AND SUBTITLE}

Final report

Flow Table Study of Cook Inlet, Alaska

5a. CONTRACT NUMBER

5b. GRANT NUMBER

5c. PROGRAM ELEMENT NUMBER

\section{AUTHOR(S)}

Steven A. Hughes, Gian-Marco Pizzo

5d. PROJECT NUMBER

5e. TASK NUMBER

5f. WORK UNIT NUMBER

32935

\section{PERFORMING ORGANIZATION NAME(S) AND ADDRESS(ES)}

8. PERFORMING ORGANIZATION REPORT NUMBER

U.S. Army Engineer Research and Development Center

Coastal and Hydraulics Laboratory

ERDC/CHL TR-03-6

3909 Halls Ferry Road, Vicksburg, MS 39180-6199;

Department of Civil and Environmental Engineering

University of California at Berkeley, Berkeley, CA 34720

9. SPONSORING / MONITORING AGENCY NAME(S) AND ADDRESS(ES)

10. SPONSOR/MONITOR'S ACRONYM(S)

U.S. Army Corps of Engineers

Washington, DC 20314-1000

11. SPONSOR/MONITOR'S REPORT NUMBER(S)

\section{DISTRIBUTION / AVAILABILITY STATEMENT}

Approved for public release; distribution is unlimited.

\section{SUPPLEMENTARY NOTES}

\section{ABSTRACT}

Complex flow patterns in upper Cook Inlet in the vicinity of the Port of Anchorage were simulated using a flow table. Initially, two idealized models of the inlet were constructed with terraced layers representing the bathymetry. Encouraging test results prompted construction of a model with three-dimensional bathymetry. Both ebb and flood maximum tide flows could be examined by reversing the models on the flow table. The intriguing flow patterns of Cook Inlet were visualized using floating particles and by dye injection. Strong horizontal and vertical mixing was evident through the model, particularly in the lee of headlands. The flow table models indicated that deposition of fine sediment at the Port of Anchorage may be caused, in part, by ebb flow separation at the upstream Cairn Point that creates a low-flow region at the port. Experimentation with the models also provided insight into potential improvements to dredge material disposal practices.

Theoretical analyses examined potential turbulence scale effects caused by geometric model distortion, and extensive experiments were conducted on the flow table to gauge the scale effects. Various jet flow geometries were tested at different model distortions, and velocity measurements in the region of flow separation were compared and evaluated. Results indicated that turbulence generated by

(Continued)

\begin{tabular}{|c|c|c|c|c|c|}
\hline \multicolumn{2}{|l|}{ 15. SUBJECT TERMS } & Cook Inlet & \multicolumn{3}{|c|}{ Geometrical distortion } \\
\hline \multicolumn{2}{|l|}{ Alaska } & Flow table & \multicolumn{3}{|c|}{ Port of Anchorage } \\
\hline \multicolumn{2}{|l|}{ Alaska District } & Physical model & & & \\
\hline \multicolumn{3}{|c|}{ 16. SECURITY CLASSIFICATION OF: } & $\begin{array}{l}\text { 17. LIMITATION } \\
\text { OF ABSTRACT }\end{array}$ & $\begin{array}{l}\text { 18. NUMBER } \\
\text { OF PAGES }\end{array}$ & $\begin{array}{l}\text { 19a. NAME OF RESPONSIBLE } \\
\text { PERSON }\end{array}$ \\
\hline $\begin{array}{l}\text { a. REPORT } \\
\text { UNCLASSIFIED }\end{array}$ & $\begin{array}{l}\text { b. ABSTRACT } \\
\text { UNCLASSIFIED }\end{array}$ & $\begin{array}{l}\text { c. THIS PAGE } \\
\text { UNCLASSIFIED }\end{array}$ & & & $\begin{array}{l}\text { 19b. TELEPHONE NUMBER (include } \\
\text { area code) }\end{array}$ \\
\hline
\end{tabular}




\section{4. (Concluded)}

vertical edges and manifested primarily in the horizontal plane had no distorted models where the vertical and horizontal turbulence had similar strength such as occurs with flow separation at a sloping edge. The difference was most noticeable near the bottom, whereas comparisons near the free surface were reasonable. The scale effect appeared to be localized around the boundary of the jet. 